\title{
STRESS-PATH DEPENDENCY OF RESILIENT BEHAVIOUR OF GRANULAR MATERIALS
}

\author{
by \\ YING LIU, B.ENG, M.ENG
}

\begin{abstract}
A thesis
Submitted to the School of Graduate Studies

in Partial Fulfillment of the Requirements for

the Degree

Doctor of Philosophy
\end{abstract}

McMaster University

CCopyright by Ying Liu, January 2010 
DOCTOR OF PHILOSIPHY (2010)

(Civil Engineering)
McMaster University

Hamilton, Ontario

TITLE: Stress-path Dependency of Resilient Behaviour of Granular Materials AUTHOR: $\quad$ Ying Liu

B.Eng. M.Eng (Huazhong University of Science and Technology)

SUPERVISOR: Peijun Guo and Dieter Stolle

NUMBER OF PAGES: $\quad$ xvii, 191 


\section{ABSTRACT}

The resilient modulus and Poisson's ratio of granular materials used in flexible pavement structures is highly nonlinear, stress/strain path and direction-dependent. Resilient properties are very important for realistic flexible pavement design. By far, several important aspects, such as the effect of stress path, major principal stress rotation, initial stress state and inherent fabric, on the cross-anisotropic resilient properties are not fully understood. The main objective of this thesis was to study the cross-anisotropic resilient properties of granular materials along various stress paths from both initial isotropic and initial anisotropic stress states. Extensive resilient modulus stress path tests were performed for this purpose.

In this research, the resilient behaviour of the test material for initial isotropic stress states along various stress paths was investigated first, with particular interest in the stress/strain path dependency. New resilient modulus equations were then developed, by taking into account the effect of confining pressure and resilient strains. These equations can be used to estimate the cross-anisotropic resilient modulus corresponding to initial isotropic stress states. Selection of Poisson's ratio was also investigated.

The effect of initial stress state on the resilient responses was studied through a series of stress path tests with constant confining pressure and constant vertical stress, respectively. Based on the experimental findings, revised equations for resilient modulus and Poisson's ratio were proposed to account for the effect of ratio of initial horizontal stress to vertical stress $\left(K_{i n i}\right)$. The proposed equations can predict the cross-anisotropic 
resilient properties for various stress paths corresponding to initial isotropic/anisotropic stress conditions.

In order to provide a more comprehensive insight into the complex resilient properties of granular materials along different stress/strain paths for various initial stress states, a micromechanics approach was introduced to back-calculate the degree of fabric anisotropy. The variations of fabric with stress path, initial stress state and final stress state (i.e., state which corresponds to the peak stress during cyclic loading) were investigated. To provide a reliable prediction of initial/inherent fabric anisotropy and fabric evolution in constitutive $\mathrm{s}$ with embedded microstructure, an evolution law of fabric anisotropy was developed. 


\section{ACKNOWLEDGEMENTS}

I would like to express my sincere and grateful thanks to my supervisors, Dr. Peijun Guo and Dr. Dieter Stolle. Their guidance and support during these years of research have been invaluable. I am grateful for their exceptional patience and encouragement. Their directions, advice and expertise were helpful in accomplishing this research. Their insight and dedication in research and supervision are truly impressive.

I am grateful to Dr. John Emery for his invaluable help, encouragement and also for providing the insight into the research.

I would like to thank Dr. Samir Chidiac and Dr. Mukesh Jain for their valuable advice and excellent service as my supervisory committee members.

The laboratory work was one of the major components of this study. The successful completion of this testing program was due in part to the assistance of Mr. Peter Koudys. I would like to express my heartfelt appreciation to him for his tireless support.

Finally, I would like to thank my parents and husband for all the love, encouragement and support throughout these years. 


\section{TABLE OF CONTENTS}

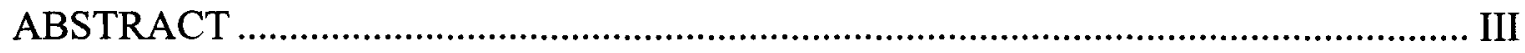

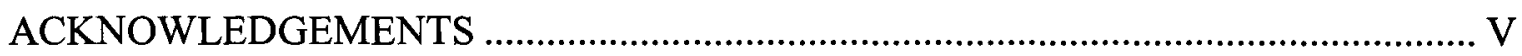

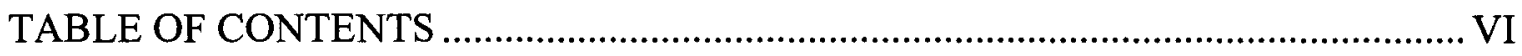

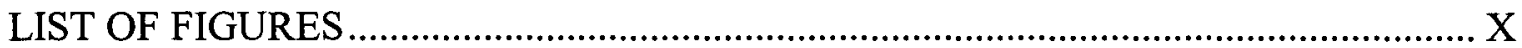

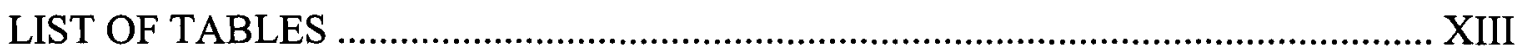

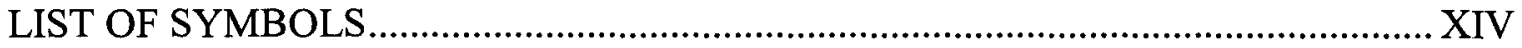

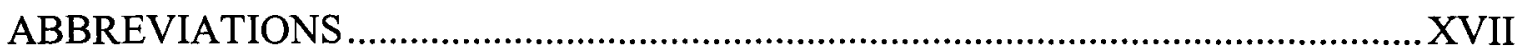

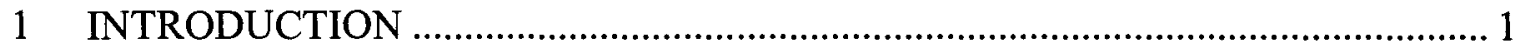

1.1 BACKGROUND AND MOTIVATIONS .................................................... 1

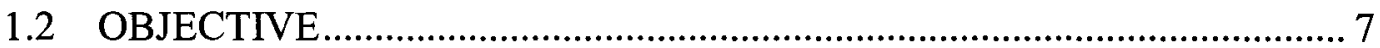

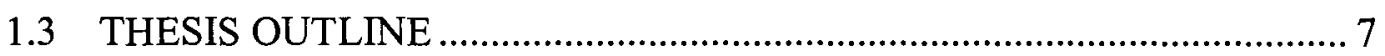

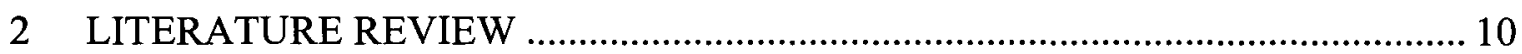

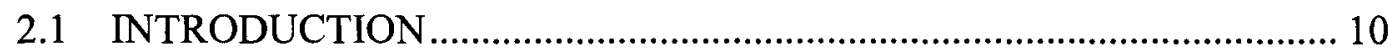

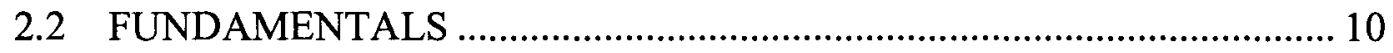

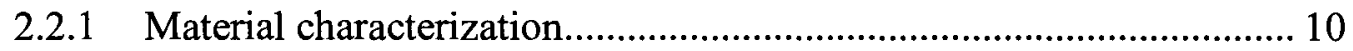

2.2.2 Resilient deformation characteristics of granular materials................. 12

2.3 FACTORS AFFECTING RESILIENT PROPERTIES ............................. 15

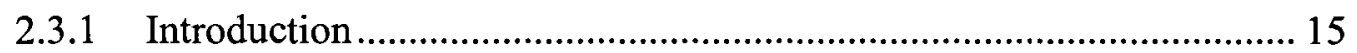

2.3.2 Physical properties ........................................................................... 16

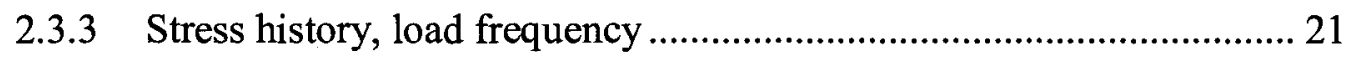

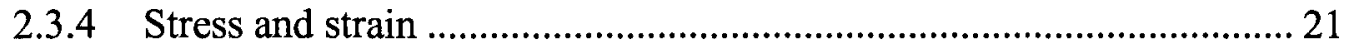

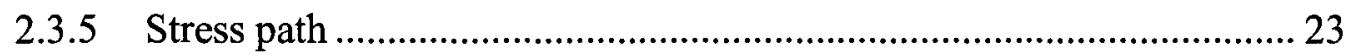

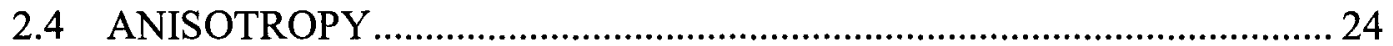

2.5 EQUATIONS FOR RESILIENT MODULUS AND POISSON'S RATIO 32

2.5.1 Resilient modulus as a function of stresses........................................... 32

2.5.2 Poisson's ratio as a function of stresses .............................................. 38 


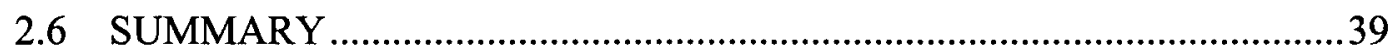

3 TESTING MATERIALS, EQUIPMENT, DESIGN AND PROCEDURES ..............41

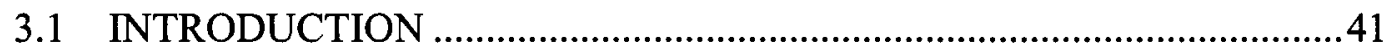

3.2 EXPERIMENTAL DESIGN METHODOLOGY …..................................41

3.2.1 Paths of loading and unloading .......................................................... 41

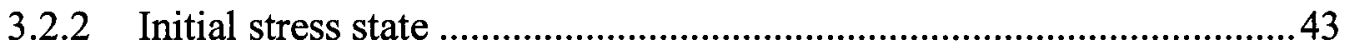

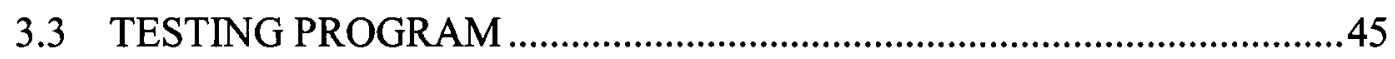

3.4 DESCRIPTION OF TESTING EQUIPMENT .........................................46

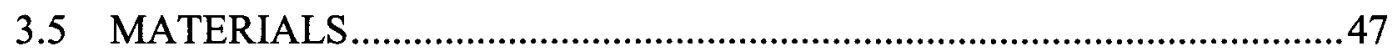

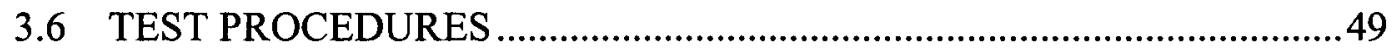

3.6.1 Sample preparation ...........................................................................49

3.6.2 Specimen setup ..................................................................................

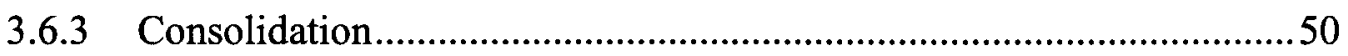

3.6.4 Cyclic loading tests...............................................................................

3.7 PERFORMANCE AND REPEATABILITY .........................................51

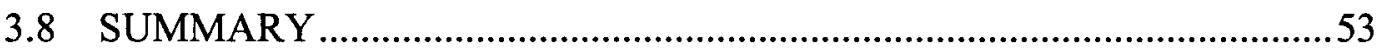

4 RESILIENT BEHAVIOUR FOR ISOTROPIC CONSOLIDATION ........................54

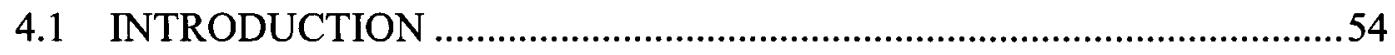

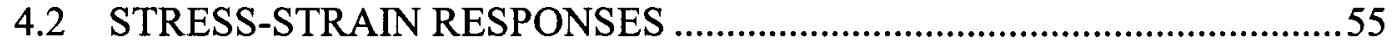

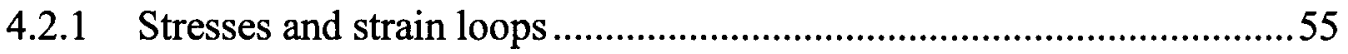

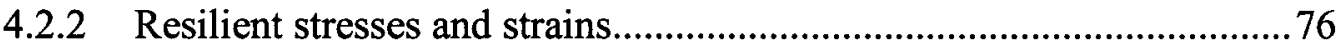

4.2.3 Effect of initial confining pressures.................................................... 81

4.3 DETERMINATION OF CROSS-ANISOTROPIC PROPERTIES ............84

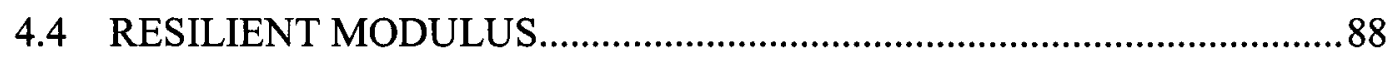

4.4.1 Resilient modulus and its dependency on stress.................................8

4.4.2 Resilient modulus and its dependency on resilient strain.....................95

4.5 RESILIENT POISSON'S RATIO AND STRESS ….................................101

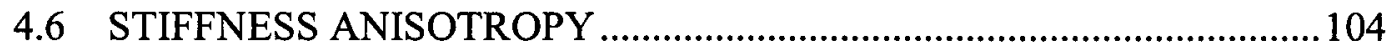

4.6.1 Stiffness anisotropy and deformation ................................................. 104 
4.6.2 Stiffness anisotropy and stress ....................................................... 106

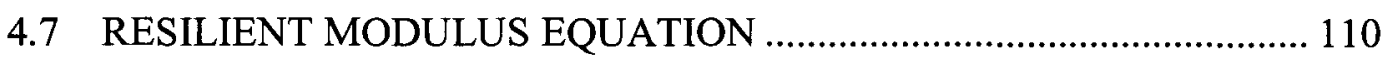

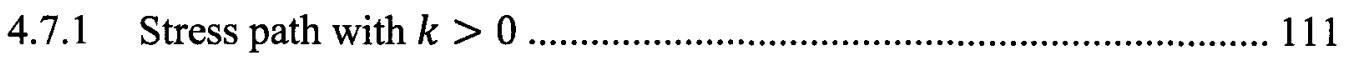

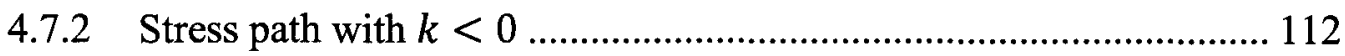

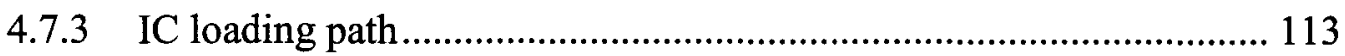

4.8 SELECTION OF RESILIENT POISSON'S RATIO ............................... 115

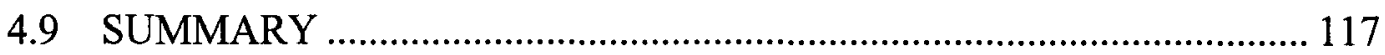

5 RESILIENT BEHAVIOUR FOR ANISOTROPIC CONSOLIDATION ............... 119

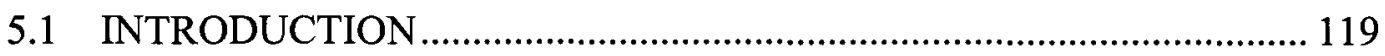

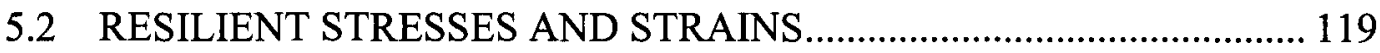

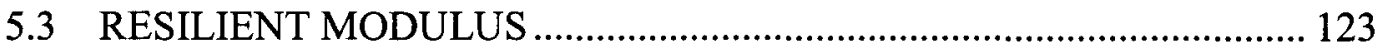

5.3.1 Resilient modulus and it's dependency on stress .............................. 123

5.3.2 Resilient modulus and it's dependency on resilient strain ................. 128

5.4 STIFFNESS ANISOTROPY AND STRESS.......................................... 129

5.4.1 Stress-induced anisotropy .............................................................. 129

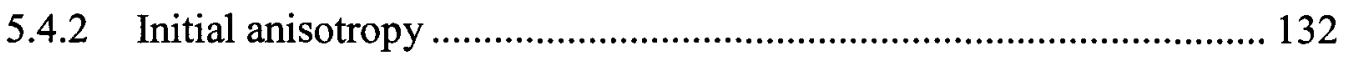

5.5 REVISED RESILIENT MODULUS EQUATION .................................. 133

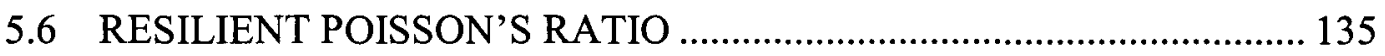

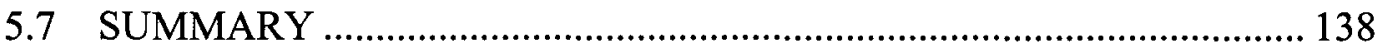

6 ANISOTROPY WITHIN MICROMECHANICAL FRAMEWORK ....................... 140

6.1 FABRIC ANISOTROPY: MICROMECHANICS ..................................... 140

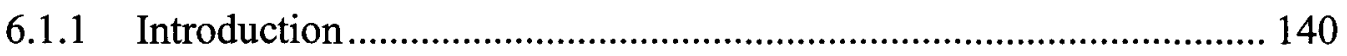

6.1.2 Fundamentals: Description of anisotropic microstructure ................. 141

6.1.3 Macro-Micro relationship ............................................................ 143

6.2 A COMPLIANCE MATRIX FOR GRANULAR ASSEMBLY ............. 143

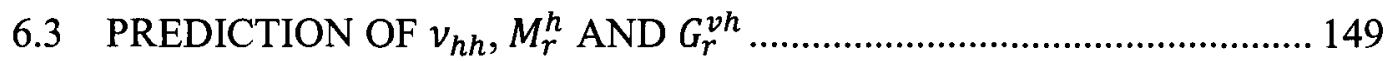

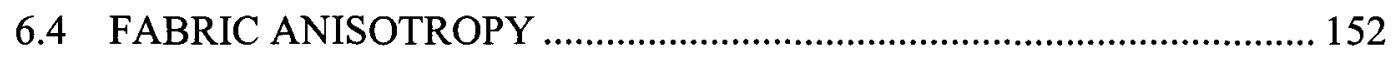

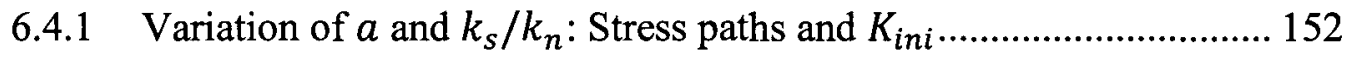

6.4.2 Evolution of fabric anisotropy: fabric anisotropy and stress ............. 157 


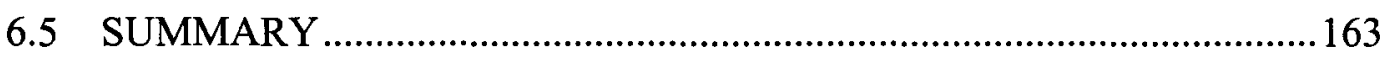

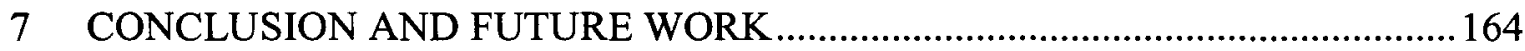

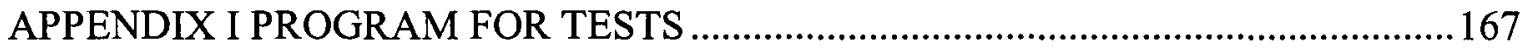

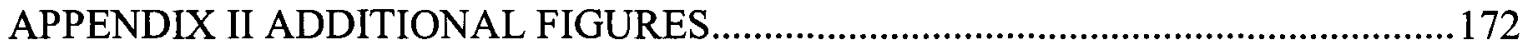
APPENDIX III COMPONENTS OF A COMPLIANCE MATRIX ..............................183

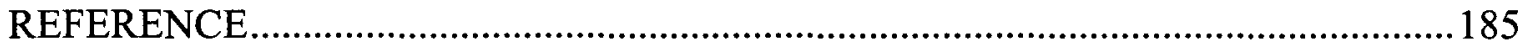




\section{LIST OF FIGURES}

Fig. 1.1 Stress changes due to rolling wheel load (Lekarp, 2000) ................................... 3

Fig. 2.1 Strains during one load cycle and definition of resilient modulus....................... 11

Fig. 2.2 Deformation characteristics for repeated CCP tests ............................................ 13

Fig. 2.3 Deformation characteristics for repeated CCP tests ………............................... 14

Fig. 2.4 Contour model in $p-q$ space (Brown and Pappin, 1985)..................................... 37

Fig. 3.1 Actual rolling wheel stress paths from the National Airport Pavement Tests ..... 42

Fig. 3.2 Schematic of stress paths in testing depicted in stress spaces............................... 43

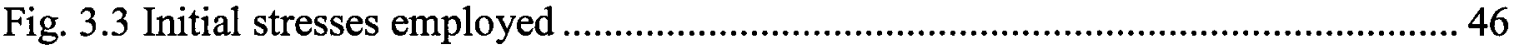

Fig. 3.4 Wykeham Farrance Dynamic Hollow Cylinder Apparatus .................................. 47

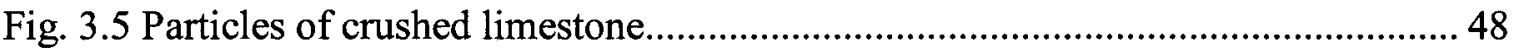

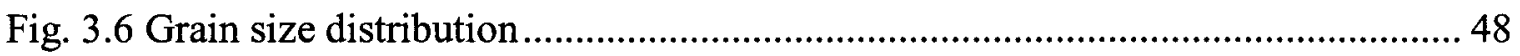

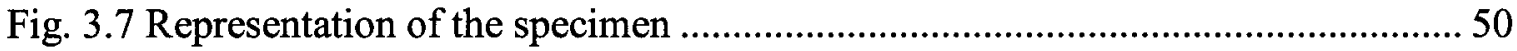

Fig. 3.8 Repeatability of stress path tests ...................................................................... 52

Fig. 4.1 Predicted responses of a linear isotropic material................................................ 57

Fig. 4.2 Predicted responses of a nonlinear isotropic material........................................... 61

Fig. 4.3 Predicted responses of a linear anisotropic material ............................................... 66

Fig. 4.4 The first cycle response loop of test specimen ..................................................... 70

Fig. 4.5 Strain loops for various stress paths.................................................................... 76

Fig. 4.6 Resilient stresses and resilient strains ................................................................. 77

Fig. 4.7 Resilient strain components for all stress paths .................................................... 79

Fig. 4.8 Stress ratios applied and strain ratios for different stress paths ........................... 81

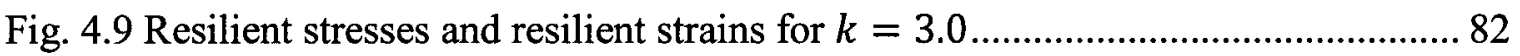

Fig. 4.10 Resilient stresses and resilient strains for $k=-1.5$........................................ 83

Fig. 4.11 Resilient stresses and resilient strains for IC loading ....................................... 84

Fig. 4.12 Stress strain relationships for CCP test .............................................................. 85

Fig. 4.13 Stress strain relationships for triaxial test ......................................................... 85 
Fig. 4.14 Resilient moduli and stress increments for stress paths $k>0$........................90

Fig. 4.15 Resilient moduli and stress increments for stress paths $k<0$.........................91

Fig. 4.16 Resilient moduli and stress increments for IC compression.............................92

Fig. 4.17 Vertical resilient moduli and stress increments for stress paths $k>0$.............93

Fig. 4.18 Horizontal resilient moduli and stress increments for stress paths $k>0$.........93

Fig. 4.19 Vertical resilient moduli and stresses for stress paths $k<0$............................94

Fig. 4.20 Horizontal resilient moduli and stressesfor stress paths $k<0$.........................95

Fig. 4.21 Resilient moduli and resilient strain components for stress paths $k>0$..........96

Fig. 4.22 Resilient moduli and resilient strain components for stress paths $k<0$..........99

Fig. 4.23 Stress and strain components for IC loading paths........................................... 101

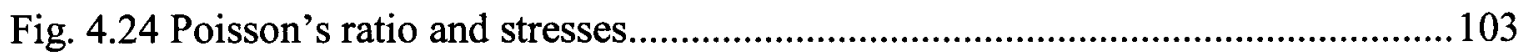

Fig. 4.25 Stiffness anisotropy and deformation characteristics .......................................105

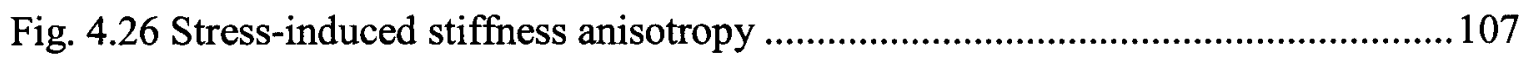

Fig. 4.27 Predicted and calculated modulus ratio for stress paths with $k \neq 0$................ 108

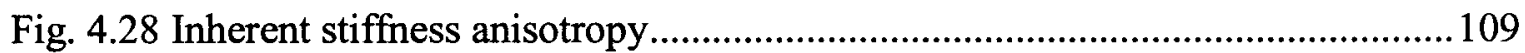

Fig. 4.29 Predicted and measured resilient moduli from positive stress paths ................112

Fig. 4.30 Predicted and measured resilient moduli from negative stress paths ............... 113

Fig. 4.31 Predicted and measured resilient moduli from IC loading paths......................114

Fig. 4.32 Predicted and measured Poisson's ratio for $k=2.0$ and $k=3.0 \ldots \ldots \ldots \ldots \ldots \ldots . . .116$

Fig. 5.1 Variation of void ratios before and after anisotropic consolidation ....................120

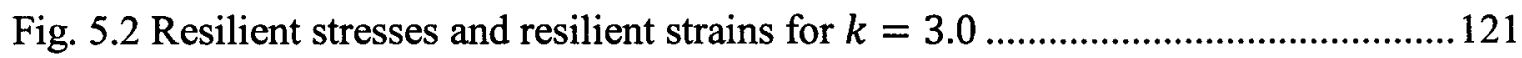

Fig. 5.3 Resilient stresses and resilient strains for $k=-1.5 \ldots \ldots \ldots \ldots \ldots \ldots \ldots \ldots \ldots \ldots \ldots \ldots \ldots . . . . .122$

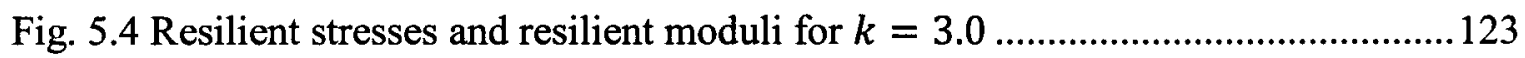

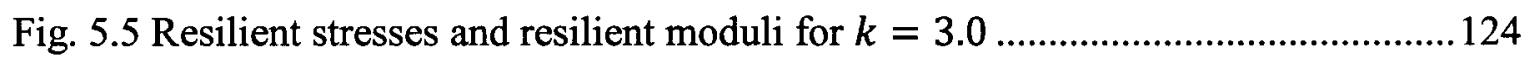

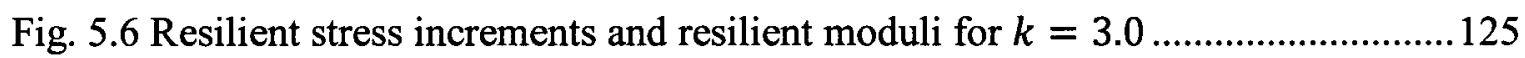

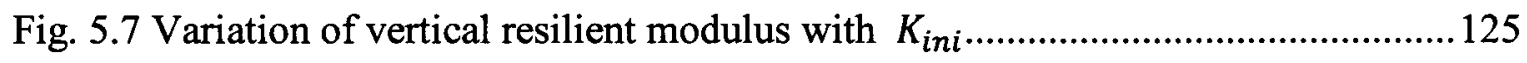

Fig. 5.8 Resilient stresses and resilient moduli for $k=-1.5 \ldots \ldots \ldots \ldots \ldots \ldots \ldots \ldots \ldots \ldots \ldots \ldots \ldots \ldots . . . . .126$

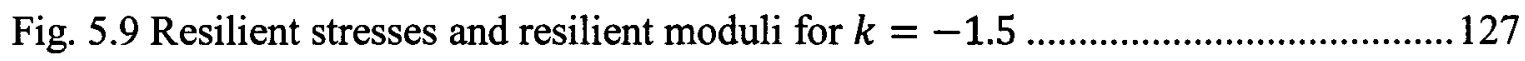

Fig. 5.10 Resilient strains and resilient moduli for $k=3.0 \ldots \ldots \ldots \ldots \ldots \ldots \ldots \ldots \ldots \ldots \ldots \ldots \ldots \ldots \ldots . . . . .128$

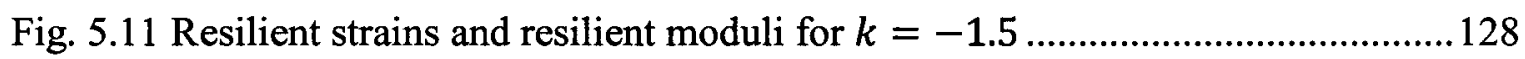


Fig. 5.12 Dependency of stiffness anisotropy on stress states .................................... 130

Fig. 5.13 Stress-induced stiffness anisotropy obtain from test Series A and B ............. 132

Fig. 5.14 Comparison between predicted and measured resilient moduli........................ 135

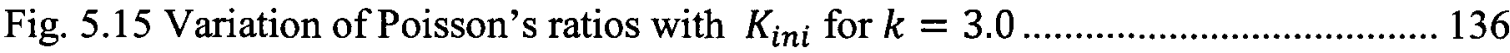

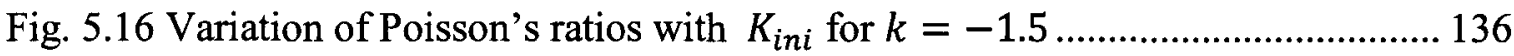

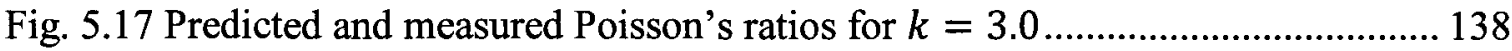

Fig. 6.1 (a) Local coordinate at inter particle contact (b) Definition of branch vector ... 141

Fig. 6.2 Distribution of contact orientations with different value of fabric anisotropy .. 142

Fig. 6.3 Variation of stiffness ratio with degree of fabric anisotropy ........................... 147

Fig. 6.4 Variation of Poisson's ratios ........................................................................... 148

Fig. $6.5 a$ and $k_{s} / k_{n}$ obtained from micromechanics model and G-H assumption ........ 151

Fig. $6.6 v_{h h}$ and $M_{r}^{h}$ obtained from micromechanics model and the G-H assumption... 151

Fig. 6.7 Stiffness anisotropy and fabric anisotropy......................................................... 153

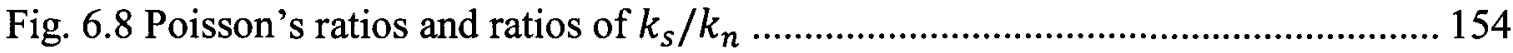

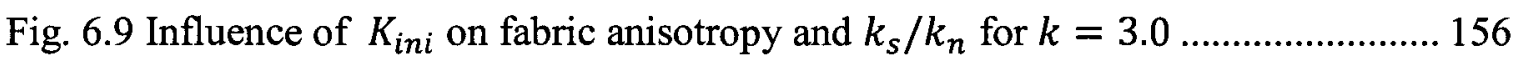

Fig. 6.10 Influence of $K_{i n i}$ on fabric anisotropy and $k_{s} / k_{n}$ for $k=-1.5 \ldots \ldots \ldots \ldots \ldots \ldots \ldots . . .157$

Fig. 6.11 Influence of stress on fabric: initial isotropic stress state ............................. 159

Fig. 6.12 Comparison of $F_{1}$ between micromechanics model and proposed approach .. 161

Fig. 6.13 Influence of stress on fabric: isotropic/anisotropic initial stress state ............. 162

Fig. AI. 1 Sample of stresses and deformation measured in tests .................................. 171

Fig. AII. 1 Resilient moduli and stress increments for stress paths $k>0$.................... 172

Fig. AII. 2 Resilient moduli and stress increments for stress paths $k<0$..................... 173

Fig. AII. 3 Vertical resilient moduli and stress increments for stress paths $k>0$........ 174

Fig. AII. 4 Horizontal resilient moduli and stress increments for stress paths $k>0$.... 175

Fig. AII. 5 Vertical resilient moduli and stress increments for stress paths $k<0 \ldots \ldots \ldots 176$

Fig. AII. 6 Horizontal resilient moduli and stress increments for stress paths $k<0 \ldots . .177$

Fig. AII. 7 Resilient moduli and resilient strain components for stress paths $k>0$..... 178

Fig. AII. 8 Resilient moduli and resilient strain components for stress paths $k<0$..... 180

Fig. AII. 9 Stress-induced stiffness anisotropy ……….................................................. 182 


\section{LIST OF TABLES}

Table 2.1 Previous investigations on cross-anisotropy in soils. ....................................27

Table 2.2 Resilient modulus equations ...............................................................................35

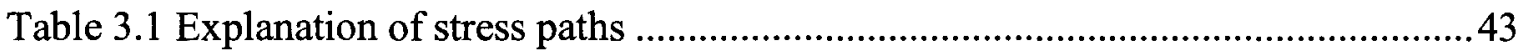

Table 4.1 Samples calculation of cross-anisotropic properties...........................................8 88

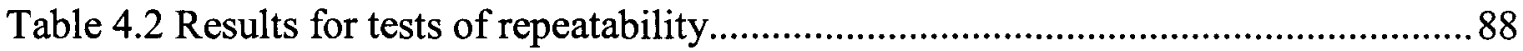

Table 6.1 The local to global coordinate system ........................................................... 144

Table AI. 1 Program for test Series A............................................................................167

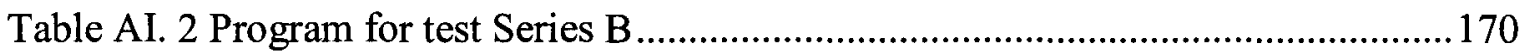

Table AI. 3 Specifications of testing equipment............................................................. 171 
$\varepsilon_{h} \quad$ horizontal strain

$\varepsilon_{h, r} \quad$ recoverable horizontal strain

$\varepsilon_{i} \quad$ strain in any particular direction $i$

$\varepsilon_{p} \quad$ permanent strain

$\varepsilon_{q}=2\left(\varepsilon_{1}-\varepsilon_{3}\right) / 3$, shear strain

$\varepsilon_{r} \quad$ lateral/horizontal strain

$\varepsilon_{v} \quad$ vertical/axial strain

$\varepsilon_{v o l} \quad$ volume strain

$\varepsilon_{v, r} \quad$ recoverable vertical/axial strain

$\varepsilon_{h, r} \quad$ recoverable lateral/horizontal strain

$\gamma=\varepsilon_{1}-\varepsilon_{3}$, shear strain

$\iota=\Delta \sigma_{v} / \Delta \sigma_{h}$, stress ratio

$\phi \quad$ friction angle

$\mu_{h v}=E_{v} /\left(2 v_{v h} F_{h}\right)$

$\Omega \quad$ unit sphere, $d \Omega$ : the solid angle of a sphere

$\sigma_{1}, \sigma_{3}$ major and minor principal stress, respectively

$\sigma_{a} \quad$ repeated axial stress

$\sigma_{c} \quad$ cell/confining pressure

$\sigma_{c}^{\prime} \quad$ confining pressure at the final stress state

$\sigma_{d} \quad$ repeated deviator stress

$\sigma_{d, f} \quad$ deviator stress at failure

$\sigma_{h} \quad$ horizontal/lateral stress

$\sigma_{h}^{i} \quad$ initial lateral stress

$\sigma_{i} \quad$ stress in any particular direction $i$

$\sigma_{v} \quad$ vertical stress

$\tau \quad$ shear stress

$\tau_{\text {oct }}=1 / 3 \sqrt{\left(\sigma_{1}-\sigma_{2}\right)^{2}+\left(\sigma_{2}-\sigma_{3}\right)^{2}+\left(\sigma_{1}-\sigma_{3}\right)^{2}}$, octahedral shear stress

$v \quad$ Poisson's ratio

$v_{h h} \quad$ Poisson's ratio for horizontal strain due to horizontal strain at right angle

$v_{v h} \quad$ Poisson's ratio for horizontal strain due to vertical strain

$\theta \quad=\sigma_{1}+\sigma_{2}+\sigma_{3}$, bulk stress 


\section{ABBREVIATIONS}

AASHTO American Association of State Highway Officials

AC Asphalt concrete

CCP Constant confining pressure

CTC Conventional triaxial compression

CTE Conventional triaxial extension

FAA Federal Aviation Administration

HCA Hollow Cylinder Apparatus

IC Isotropic compression

IMACS Integrated Multi-Axis Control System

LTPP Long Term Pavement Performance

LVDT Linear Variable Differential Transducer

NAPTF National Airport Pavement Test Facility

PL Proportional loading

REV Representative elementary volume

VCP Variable confining pressure 


\section{INTRODUCTION}

\subsection{BACKGROUND AND MOTIVATIONS}

For flexible pavements, the unbound granular base/subbase serves to provide adequate support to the upper asphalt concrete (AC) layer and distribute the load from surface traffic, as well as to keep the subgrade from excessive deformation. A well compacted, high quality granular base can reduce the thickness of the upper AC layer, and extend the service life of the pavement, which in turn reduces the cost of construction and maintenance. In areas where rigid pavements are generally not used, a thin $\mathrm{AC}$ layer over a well compacted, high quality granular base layer is the most economical and effective type of pavement. Since the shear stresses in granular base/subbase layers are usually far less than the shear strength of the materials, the overall structural performance of pavement greatly depends on the mechanical properties of granular base/subbase layers, particularly on their resilient modulus and ability to resist permanent deformation.

The tools available for pavement design have significantly advanced over several decades. Specifically, computerized numerical modelling techniques are now available for complex analysis and design. However, sophisticated and reliable analysis and design rely heavily on accurate characterization of the pavement materials for design inputs. As a result, a proper understanding of the mechanical properties of granular base/subbase materials, particularly the deformation prior to failure and the resilient responses, is a 
prerequisite for a proper, rational design of flexible pavement structures.

The characterization of granular base/subbase materials for pavement design is not a trivial task, for the following reasons:

(1) The resilient response of granular materials is highly nonlinear and generally depends on both stress state and stress path. Under the application of a single rolling wheel load, appropriately designed and constructed pavements respond mainly in a resilient manner, with the plastic/irrecoverable strains being negligible compared to the resilient strains for each stress repetition. However, the accumulated irrecoverable deformation may not be neglected given a large number of repeated load applications, even though the shear stress level is far below the shear strength of the material. Owing to the initial compaction during construction, the base/subbase experiences plastic deformation, hopefully "shaking down" to an elastic response. The granular layers are designed to have resilient responses under subsequent traffic loading, provided the load does not exceed the shakedown limit of the material. If the stress level induced by traffic load is increased above the shakedown limit then additional plastic deformation continues to occur.

(2) Subgrade soils and the granular materials in a pavement structure are subjected to large numbers of load repetitions at stress levels well below their shear strength (Brown, 1996), and stress histories induced in a pavement due to a rolling wheel load are quite complex. Figure 1.1 illustrates the stress pattern due to a moving wheel load, which is transient and repetitive. For an element in the system, the stress pulses consist of vertical, horizontal, and shear components, which result in the rotation of the principal 
stresses. The stress path applied in the laboratory, however, is usually limited by the equipment and cannot reproduce the in-situ stress paths existing in the pavement, which in turn can lead to unrealistic estimates of resilient modulus for pavement design.

(3) The particle sizes of soils used for pavement construction vary over a wide range. These materials usually have more uncertainty and variability compared with "textbook" soils. Large size samples (representative volume element) are required in the laboratory to obtain representative behaviours of the materials. Therefore, it is difficult to define a simple rule for the resilient behaviour of the granular material specimen, since the global and local deformation of the specimen may be different under cyclic loading.
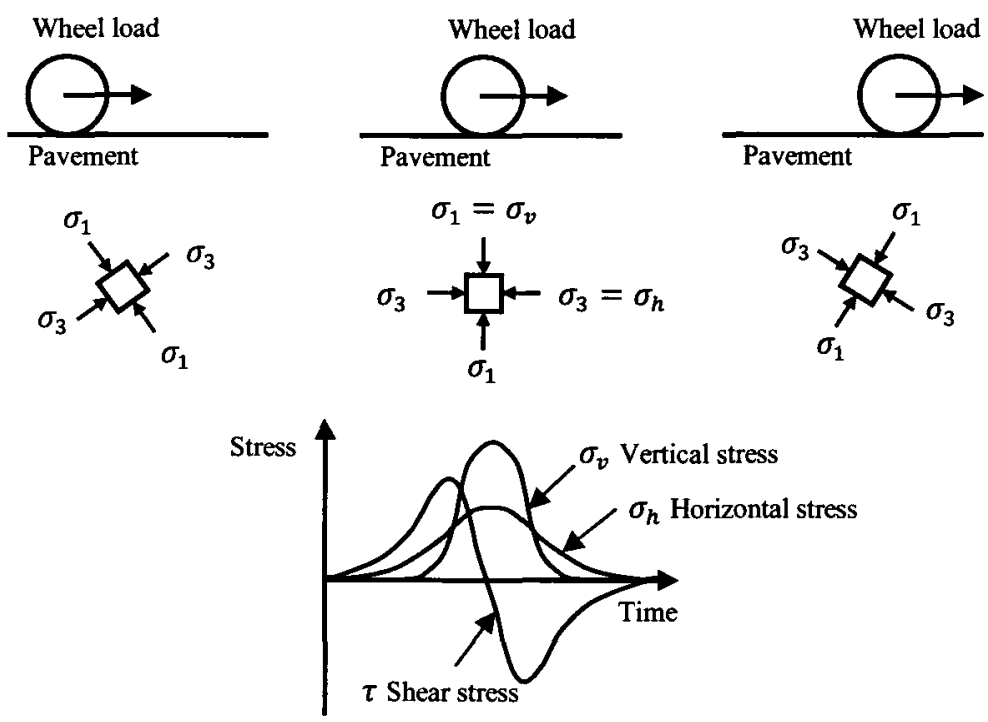

Fig. 1.1 Stress changes due to rolling wheel load (Lekarp, 2000)

A granular material can be considered as a collection of discrete particles with different types of interaction among particles, including interparticle friction and interlocking. On one hand, the particulate nature of the material leads to its nonlinear 
behaviour. On the other hand, its non-spherical particle shape and the fabric anisotropy as a result of disposition history and past loading, cause directional variation of shear resistance (strength) and stiffness; i.e., anisotropy in strength and stiffness. Anisotropy of soil can be inherent anisotropy or stress-induced anisotropy (Casagrande and Carillo 1994). Normally, inherent anisotropy is produced through sedimentation of naturally deposited soils, or artificially prepared/compacted materials. Stress-induced anisotropy is induced by subsequent loading, which causes particle rearrangement through movement and rotation towards some preferential directions. Alternatively, one may use the terms initial and subsequent anisotropy to indicate the sequence of the events. For granular material in a pavement system, the manner that the granular layers are laid, the method of compaction and the subsequent traffic loading, all influence the resilient response of pavement. The directional variation of stiffness relative to loading directions may have significant effect on the performance of the system. From a theoretical point of view, the anisotropy must be included in any analysis due to its effect on the development of stresses and strains.

Over the years, numerous studies have been carried out on granular materials and subgrade soils, from which different constitutive equations have been proposed to characterize the resilient behaviour of these materials. In general, the resilient behaviour is highly nonlinear, and is affected by various factors such as stress state, moisture condition and the nature of the applied stresses. The nonlinearity has been studied extensively via cyclic triaxial tests, and the cross-anisotropy has long been recognized. However, the triaxial stress conditions are very different from those in situ, since the 
principal stress directions in triaxial tests are fixed in the vertical and horizontal directions. The effect of principal stress rotation on the resilient properties of granular materials is not fully understood and is not taken into account in the existing resilient modulus equations.

The anisotropic characteristics of clay and sand, either at small or large strains, have been investigated extensively (Zdravkovic and Jardine, 1997, Tatsuoka 2000, etc.). Zdravkovic and Jardine (1997) reported that the stiffness of soil depends strongly on the stress path direction and principal stress axis inclination. The test results for anisotropy of sand and clay, however, may not be readily applied to granular base/subbase materials in a pavement. This is because the behaviour of coarse aggregates is generally more sensitive to anisotropy than that encountered for typical sand and fine-grained soils, most likely owing to the strong interlocking of large size angular particles. Research has found that the ratio of horizontal stiffness to vertical stiffness of soil tends to increase with a decrease of grain size. For example, the ratio of horizontal to vertical modulus may vary from 1.2 to 4 for clays and from 0.5 to 1.0 for sand, while the ratio for aggregates could be as low as 0.1 (Lings et al. 2000, Chaudhary et al. 2003). Usually, naturally deposited soils develop higher horizontal stiffness than vertical stiffness. However, in a pavement, due to the orientation of the load, the long sides of granular particles tend to lay flat, leading to the vertical stiffness in the granular base being consistently higher than the horizontal stiffness. In addition, most of the current findings are on the anisotropy of sand and clay at very small strains (less than $0.001 \%$ ), while the resilient strains in granular base/subase ranges from very small to intermediate levels $(0.001 \% \sim 1 \%)$. 
At present, several important aspects, such as the effect of stress path and principal stress rotation on the resilient properties of granular materials are not taken into account in material characterization for pavement analysis due to lack of proper laboratory equipment, testing capabilities, and the uncertainty and variability of aggregate behaviour. Although cross-anisotropy has been known for a long time, it is still widely ignored in modelling of unbound granular materials. It remains unclear how these facets of behaviour should be modeled and how the pavement performance may be affected.

The need for proper characterization of granular base/subbase materials in the pavement structures has received more attention in recent years. The Guide for Mechanistic - Empirical Design of New and Rehabilitated Pavement Structures for the Design of Flexible Pavement Structures (NCHRP, 2004) has replaced the largely empirical AASHTO (American Association of State Highway Officials) 1993 design guide. The new guide requires the use of nonlinear stress-dependent resilient modulus as essential parameters to describe the behaviour of granular materials subjected to moving traffic, which represents major progress in pavement design. However, the granular material in the new design guide is still considered to be isotropic and stress-path independent. As a result, different loading stress paths and continuous principal stress rotation experienced in situ cannot be simulated. The use of isotropic resilient modulus may lead to inaccurate predictions of response of pavement structures and under-estimate the critical response of the pavement structures.

For the success of pavement design, the precise stress-strain relations are needed; more research is required on the resilient deformation behaviour of aggregates under 
general stress states.

\subsection{OBJECTIVE}

The main objective of this thesis is to study the resilient properties of granular materials used in flexible pavement structures under various loading conditions. The findings are to improve the analysis and prediction of the performance of flexible pavements, and hence provide improved design. In order to achieve this goal, the research focuses on the following tasks:

- Study the resilient behaviour of granular materials under general stress states by performing systematic testing using an advanced triaixal test apparatus. The influences of stress state and stress path are characterized with the anisotropic resilient behaviour of granular materials being explored.

- Improve, where possible, the existing resilient modulus equations for granular materials that are most important in the design of flexible pavements, and generalize more rational resilient modulus equations.

- Investigate the stiffness and fabric anisotropy of granular material as well as their influence on their resilient modulus under different stress states along various stress paths. The initial anisotropy and induced anisotropy are quantified based on measured resilient modulus. A link between anisotropy and the applied stresses is established within the framework of this research.

\subsection{THESIS OUTLINE}

Chapter 2 presents a review of the basic features of resilient behaviour of granular 
materials, particularly the nonlinear stress dependency and cross-anisotropy. The influences of various factors, including stress state and physical properties, on the resilient properties of unbound granular materials are summarized. Existing resilient modulus equations and their limitations are discussed, with some pending problems such as the influence of stress path and cross-anisotropy being presented. This comprehensive literature review provides insights and appropriate direction for the research in this thesis.

Chapter 3 describes the test set-up, material, as well as the details of the testing program. The procedure for the determination of resilient properties from stress path tests is then described. Typical experimental results are presented in order to demonstrate the consistency and repeatability of the test results.

Chapter 4 explores the resilient behaviour of granular materials along various stress paths corresponding to initial isotropic consolidation. Following a mathematical description for the determination of cross-anisotropic elastic properties, typical test results from repeated triaxial tests are presented. The effects of stress paths, stress states and resilient strains on the anisotropic resilient properties are analyzed, and stiffness anisotropy due to stresses and applied stress paths is discussed. Resilient modulus equations that take into account the effect of stress path and cross-anisotropy are developed based on the experimental results. Selection of appropriate resilient Poisson's ratios is suggested.

Chapter 5 presents the results of stress path tests for various anisotropic initial stress states. The influence of $K_{\text {ini }}$, or the initial stress state, on the anisotropic resilient properties is discussed. Based on the observation and analysis, revised resilient modulus 
and Poisson's ratio equations are proposed to account for the effect of initial stress states.

Chapter 6 investigates the fabric anisotropy of a granular material for different stress paths. Following a discussion on the effect of fabric anisotropy and stress conditions on the resilient properties, a model that describes the stress-strain behaviour of granular materials is derived from the micromechanical analysis of granular material deformation. The evolution of fabric with stresses is then investigated. A quantitative evolution law is proposed to account for the initial and subsequent fabric anisotropy.

Chapter 7 summarizes the conclusions and directions of future work. 


\section{LITERATURE REVIEW}

\subsection{INTRODUCTION}

This chapter presents a review of the literature on the resilient behaviour of unbound granular materials. The resilient deformation characteristics of granular materials are described. Factors affecting the resilient behaviours are discussed, and the anisotropy of granular materials is reviewed. Resilient modulus equations proposed by researchers, particularly the widely used equations, are presented in this chapter.

\subsection{FUNDAMENTALS}

\subsubsection{Material characterization}

Within a mechanistic framework, the structural design of pavement relies on proper characterization of the load-deformation response of the pavement materials corresponding to repeated traffic loading. Since the shear stresses developed in unbound granular material layers are usually much lower than their shear strength, the deformation characteristics are the major consideration for pavement analysis. In general, the deformation of unbound granular materials subjected to cyclic loading is characterized in terms of a resilient (recoverable) deformation and a permanent (irrecoverable) deformation, as shown in Fig. 2.1. The resilient strain is important for the short-term response of the granular base/subbase layer and the permanent strain characterizes the long-term deformation behaviour of the granular base/subbase layer. 


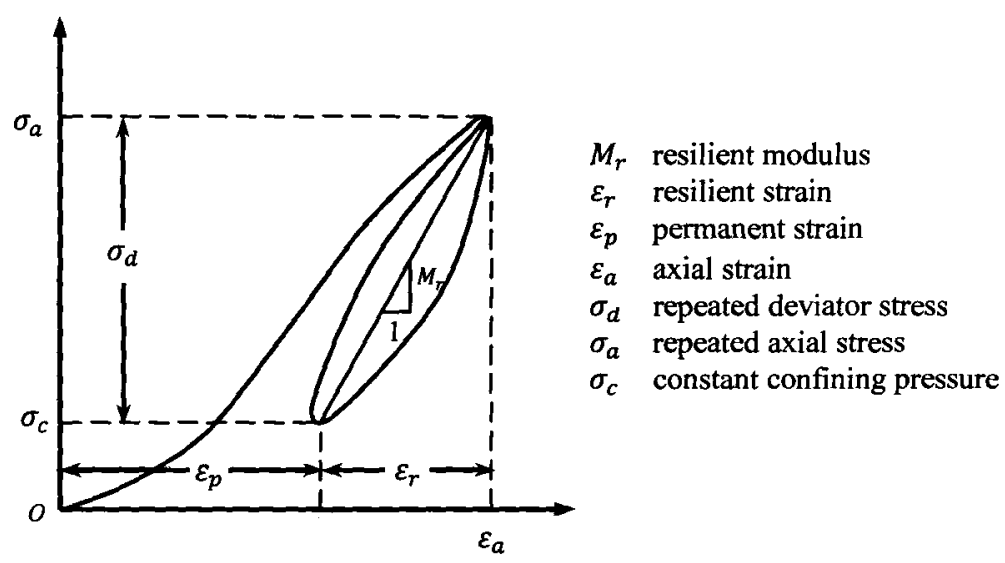

Fig. 2.1 Strains during one load cycle and definition of resilient modulus

The deformation of a granular material under cyclic loading is related to its physical properties and the amplitude of applied cyclic stress. When the cyclic stress is small, the resilient strain dominates compared to the permanent strain, thus the deformation of the granular material can be considered as stable. When the applied cyclic stress reaches a certain threshold value, the permanent strain becomes dominant and the deformation of the granular material may become unstable, which could lead to ultimate failure of the material if the cyclic stress reaches its shear strength.

The resilient response of granular material is usually defined in terms of the resilient modulus and a Poisson's ratio. For cyclic triaxial tests with constant confining pressure (CCP), Seed et al. (1962) defined the axial resilient modulus $M_{r}$ as the ratio of repeated deviator stress $\sigma_{d}$ to the recoverable axial/vertical strain $\varepsilon_{v, r}$; i.e.,

$$
\begin{gathered}
M_{r}=\frac{\sigma_{d}}{\varepsilon_{v, r}} \\
\sigma_{d}=\sigma_{1}-\sigma_{3}=\sigma_{a}-\sigma_{c}
\end{gathered}
$$

with $\sigma_{a}$ and $\sigma_{c}$ being the axial stress and confining/cell pressure, respectively. The 
resilient Poisson's ratio $v$ is defined as

$$
v=-\frac{\varepsilon_{h, r}}{\varepsilon_{v, r}}
$$

where $\varepsilon_{h, r}$ is the recoverable horizontal/lateral strain.

\subsubsection{Resilient deformation characteristics of granular materials}

AASHTO designation T307-99 specifies the standard test protocol for resilient modulus.

The stress paths applied to specimens to determine the resilient modulus are generally limited by the equipment. They can to various degrees reproduce/simulate the in-situ stress paths and stress states in soils. In a CCP triaxial test, repeated vertical pulses are applied on the test sample at a constant confining pressure, and the variation of in-situ horizontal and shear stress induced by moving traffic load cannot be reproduced.

Figures 2.2 and 2.3 show typical responses of crushed limestone under repeated axial loading ( $\sigma_{d}=25 \mathrm{kPa}$ and $50 \mathrm{kPa}$, respectively) at a constant confining pressure of $50 \mathrm{kPa}$ from this study. The sign convention is "+" for compression and "-" for extension. It can be seen that the material developed large plastic/non-recoverable deformation in the first load application. However, the recoverable/resilient deformation dominates compared to the plastic deformation in subsequent load cycles. If the repeated load is well below the strength of the material, after a large number of load cycles, the deformation in every load cycles may be "fully" recovered, which corresponds to the resilient behaviour of a material. The deformation response indicates that the granular material is nonlinear and depends on the amplitude of the applied stress. 

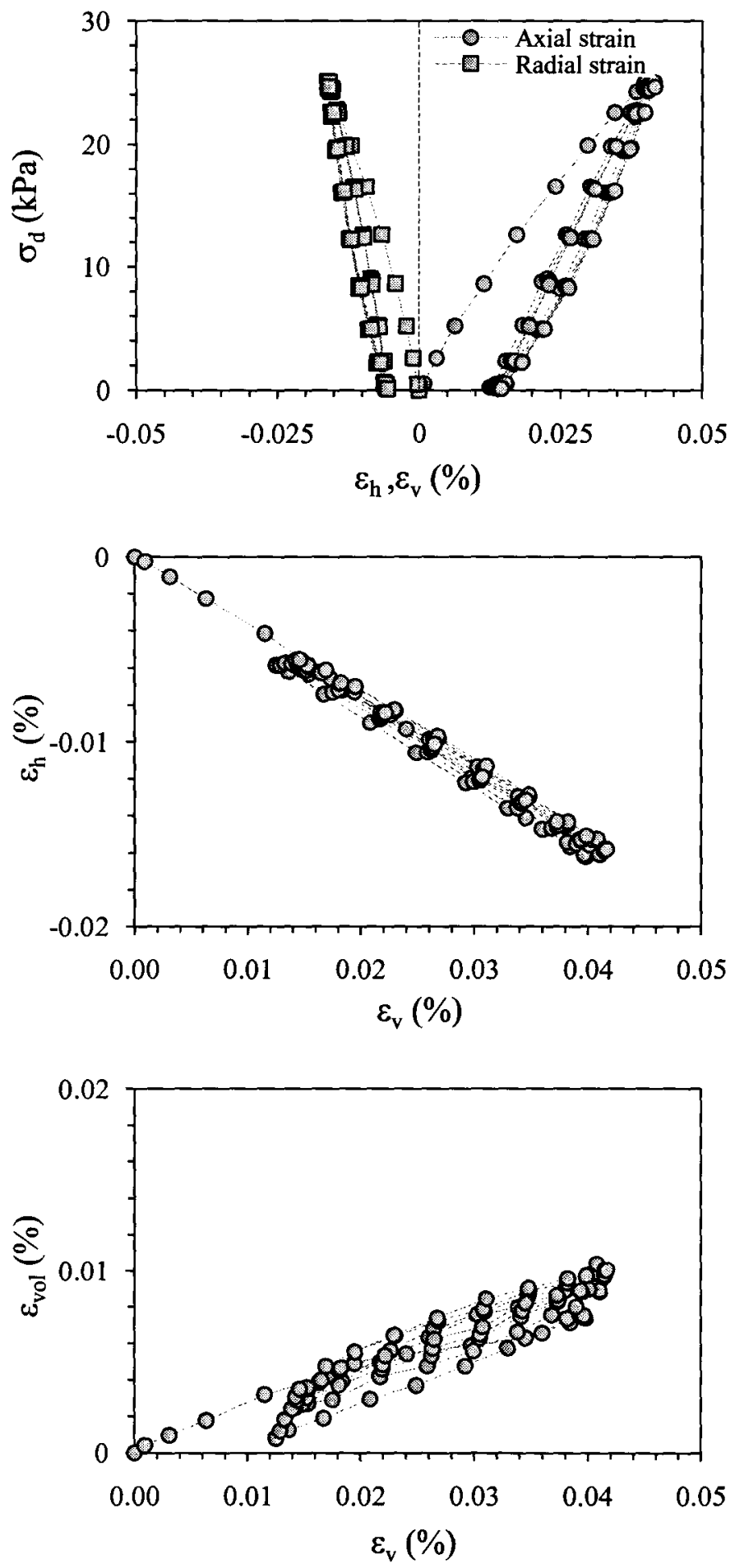

$\varepsilon_{v}:$ vertical/axial strain $\varepsilon_{h}:$ horizontal/radial strain $\varepsilon_{v o l}:$ volumetric strain

Fig. 2.2 Deformation characteristics for repeated CCP tests

$$
\sigma_{c}=50 \mathrm{kPa}, \sigma_{d}=25 \mathrm{kPa}
$$



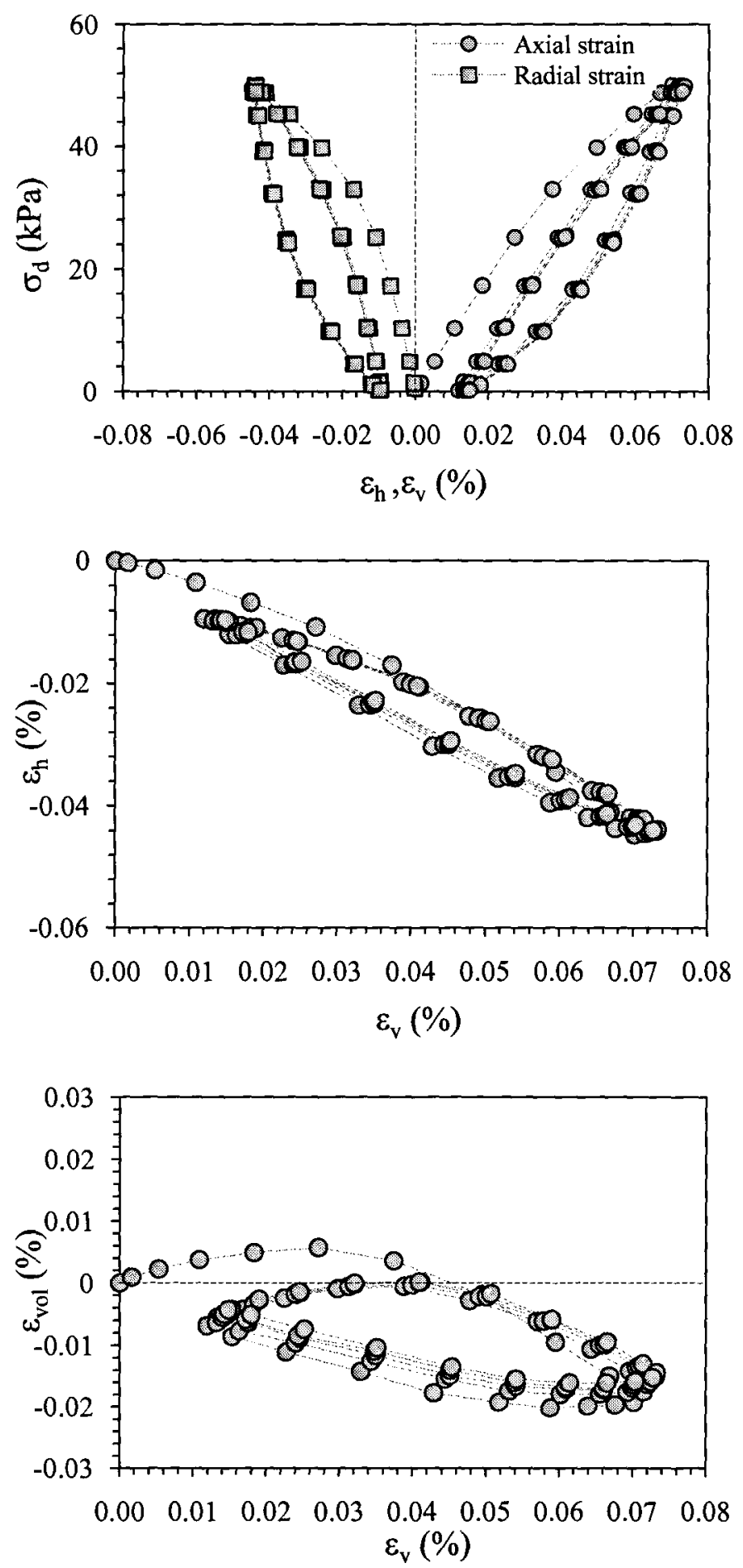

Fig. 2.3 Deformation characteristics for repeated CCP tests

$$
\sigma_{c}=50 \mathrm{kPa}, \sigma_{d}=50 \mathrm{kPa}
$$


For a well compacted material, small cyclic deviator stresses lead to very small resilient strains with the overall volume decrease, which indicates that the material becomes more compacted under cyclic shearing; see Fig. 2.2. Accordingly, one expects that stiffness of the material increases. When the amplitude of repeated cyclic deviator stress becomes larger, as shown in Fig. 2.3, both vertical and horizontal resilient strains increase. When the repeated cyclic deviator stress exceeds a certain level, an increase of specimen volume may be observed. The phenomenon of volume increase associated with particle rearrangement and induced by cyclic shearing is called resilient dilatancy. This is typical for dense compacted materials. As such, the apparent resilient Poisson's ratio is greater than 0.5 . It should be noted that resilient dilatancy does not happen for linear elastic materials.

\subsection{FACTORS AFFECTING RESILIENT PROPERTIES}

\subsubsection{Introduction}

The resilient properties of granular materials under repeated traffic load are generally influenced by factors with various degrees of importance, including stress level, moisture content, degree of compaction and the physical properties of materials.

The findings of the extent and impact of factors, such as fines content, gradation, maximum particle size, and particle shape, on the resilient response of granular materials are somewhat inconsistent. Different or even completely opposite conclusions are often reported in the literature. The discrepancies found in the literature emphasize the need for more intensive research in this area. Aspects, such as the effect of stress path and 
principal stress rotation on the resilient properties of granular materials, are generally not taken into account in material characterization for current pavement analysis due to the inability to measure the required properties because of lack of proper laboratory equipment and testing capabilities. Even though the importance of principal stress rotation on the mechanical behaviour of aggregates has long been recognized, it is still widely ignored in modelling of unbound granular materials owing to the complexity of the problem itself. An argument for neglecting the effect of principal stress rotation is that the complexity, uncertainty and variability of aggregate behaviour sometimes undermine the influence of this effect.

\subsubsection{Physical properties}

Numerous studies have shown that the resilient modulus of granular materials depends on the physical properties of the material, which include: aggregate/soil type, particle shape, gradation, density, moisture content (or the degree of saturation), fines content, etc. (Hicks 1970, Thom and Brown 1988, Brown and Selig 1991, Heydinger et. al. 1996, Kolisoja 1997). As the impact of physical properties of materials on resilient response is not a focus in this research, only major influencing factors are described below.

\section{(a) Density and compaction}

Density is an important factor for the resilient modulus of granular materials. In general, the resilient modulus is expected to increase with density, due to the fact that the particles of the material are closer and more interlocking occurs (Hicks 1970, Rada and Witczak 
1981, Yau and Von Qu, 2002). However, when other factors, such as compaction methods, moisture content, particle shape, and fines content are taken into account, the influence of density on resilient modulus is not as clear as revealed by contradictions in the literature. Thom and Brown (1988), and Brown and Selig (1991) suggested that the density has no significant effect on the resilient modulus. Hicks and Monismith (1971) showed that the resilient modulus of partially crushed aggregates tends to increase with density, while the effect of density is negligible for fully crushed aggregates. They also noted that when the fines content in the partially crushed aggregates increases, the effect of density on the resilient modulus is reduced, which shows that interparticle locking is more important than density. Besides, Barksdale and Itani (1989) suggested that the resilient modulus increases with an increase of density at low bulk stress (sum of principle stresses), while at high bulk stress, the effect of density is undermined by the effect of bulk stress. Such diverse observations indicate that the impact of a physical property on resilient behaviour of a granular material may be influenced by the interaction with other properties, and should not be evaluated separately.

The effect of density or compaction method on resilient modulus can be interpreted from the viewpoint of micromechanics. For a collection of granular particles, any variation in the degree of compaction or density will change the internal structure or particle arrangement and interaction among particles in the ensemble. It has been known that particle contacts contribute to the deformation and strength properties through interparticle cohesion and friction (Desai 2001). Any deformation of the ensemble is accompanied by particle movement (either rotation or slip at contacts or both) and 
particle deformation, including breakage. Since different compaction (dynamic, static, and kneading) methods may induce different fabric, it is expected that the resilient modulus of the same material will be affected by both density and how the density is achieved; i.e., the compaction method. It is important to recognize that laboratory compacted samples may not be representative of compaction in situ, where gyratory compaction is commonly used.

The recent study conducted by Stolle et. al. (2009) shows that compaction method has a profound influence on the moisture sensitivity and the relation of resilient modulus to bulk stress. The difference in the resilient modulus of the specimen prepared by impact method and vibration cannot be explained by the variation in density but should be considered to be the consequence of varying fabric and inter-particle locking, which depends on compaction method.

\section{(b) Moisture content}

The degree of saturation or moisture content has significant influence on the resilient properties of unbound granular materials. It has been shown that environmental wet-dry cycles also cause variations in resilient modulus. The LTPP (Long Term Pavement Performance) experimental data (Yau and Von Qu, 2002) indicates that the moisture content and density are important factors for higher strength unbound aggregate base/subbase materials. More specifically, the resilient modulus of unbound granular materials tends to decrease with increasing moisture content or the degree of saturation; see, for example, Haynes and Yoder (1963), Hicks and Monismith (1971), Dawson 
(1996), and Barksdale et al. (1997). Usually, coarse materials are not as sensitive as finegrained materials to the moisture content. On the wet side, the excess pore water pressure may develop but dissipates quickly under drained conditions due to the high permeability of the material. The effect of moisture content is more significant to the resilient response of unbound aggregates with high percentage of fines.

\section{(c) Fines}

The influence of fines on the stiffness of materials is controversial, but appears to depend on the gradation and particle angularity of aggregate. According to LTPP experimental data (Yau and Von Qu, 2002), the amount of fines is generally important for the resilient modulus of lower strength unbound aggregate base/subbase materials. Hicks and Monismith (1971) reported that increased fines content reduces $M_{r}$ for partially crushed aggregates, but increases $M_{r}$ of fully crushed aggregates. Thom and Brown (1987) observed that the resilient modulus decreases with an increase of fines.

In addition to fines content, the nature of fines, plastic or non-plastic, is considered to be key for the diverse observations regarding the variation of $M_{r}$ with respect to fines content. It has been reported that increasing plastic fines content may reduce resilient modulus. When non-plastic fines is added into clean aggregates, the initial increase of non-plastic fines tends to increase the stiffness of the material, since the fines fill the air spaces among particles and increases the number of particle contacts. However, excessive amount of fines may lubricate the interface of large-size particles and reduce the interlocking of particles, which leads to a reduction of resilient modulus. Barksdale and 
Itani (1989) showed that beyond a certain amount of fines in the aggregate, the resilient modulus decreased dramatically. Tutumluer and Seyhan (2000) proposed an optimum fines content of 7\% for crushed aggregates to achieve high stiffness. Stolle et. al. (2009) showed that the stiffening effect on resilient modulus due to confinement is less when more fines are present, which indicates that fines may act as a "lubricant".

\section{(d) Gradation and particle shape}

Gradation has some influence on the resilient modulus of material, even though it is not considered as a major influential factor for resilient modulus. Thom and Brown (1988) studied the behaviour of crushed limestone with different gradations and found that the stiffness of uniformly graded aggregates could be slightly higher than that of well-graded aggregates. Plaistow (1994) stated that gradation has an indirect effect on the resilient behaviour of granular materials since it affects the moisture sensitivity and density of the material. More specifically, well-graded materials can achieve higher densities than uniformly graded ones since small grains fill the air space between large particles. Thus, well-graded materials tend to have higher stiffness when compared to that of uniformly graded materials. Heydinger et al. (1996) and Van Niekerk et al. (2002) confirmed this for limestone and sand, crushed masonry, and crushed concrete. For gravel, Heydinger et al. (1996) found no big difference in stiffness between well-graded and uniformly graded ones. Studies by Stolle et al. (2005) and Guo et. al. (2006) have shown that aggregate gradation has a small effect on the resilient modulus of tested materials.

When the effect of particle shape is concerned, for the same gradation, crushed 
materials have higher resilient modulus than uncrushed ones; see Hicks (1970), Lam et al. (1982), Barkdale and Itani (1989), and Thom and Brown (1989). This is attributed to angular and subangular particles crushed materials providing better interlocking between particles, which yields higher values of $M_{r}$ than materials with rounded and sub-rounded particles. Lam's (1982) study showed that crushed stone aggregate yields slightly higher values of $M_{r}$ than rounded aggregate, with $M_{r}$ increasing with density, particle angularity and/or surface roughness.

\subsubsection{Stress history, load frequency}

Stress history may have some impact on the resilient behaviour of granular materials as a result of progressive densification and particle rearrangement under repeated application of stresses. However, this can be reduced by preconditioning. In addition, if the applied load is well below the strength of materials, substantial permanent deformation can be avoided, which in turn considerably reduces the influence of stress history.

The impact of load duration and frequency on the resilient behaviour of granular materials is not significant. In general, the resilient modulus of granular materials increases slightly with an increase in load frequency.

\subsubsection{Stress and strain}

Stress is the most important factor that affects the resilient properties of granular materials. In the past, owing to the limitations of equipment and testing capabilities, most resilient modulus testing was limited to CCP triaxial tests. Under CCP test conditions, the 
resilient properties of unbound granular materials are found to depend on the confining pressure/bulk stress and the applied deviator stress. Numerous studies, such as those of Hicks (1970), Uzan (1985), Sweere (1990), and Rada and Witczak (1981), revealed that the resilient modulus of granular materials generally increases with an increase in the confining pressure/bulk stress.

Compared to the effect of confining pressure/bulk stress, the influence of deviator stress on resilient modulus varies. Some researchers observed that the resilient modulus tends to decrease with an increase of deviator/shear stress. According to LTPP data (Yau and Von $\mathrm{Qu}$ 2002), the decrease of resilient modulus with deviator stress is more significant for fine-grained soils than granular base/subbase materials. Some experimental results showed that the resilient moduli of some base/subbase materials are almost independent of deviator stress (Hicks 1970, Stolle et. al. 2009). Croney and Croney (1991) observed that resilient modulus for crushed gravel can increase with an increase in deviator stress. Kolisoja (1997) and Van Niekerk (2002) argued that for coarse materials, when the applied deviator stress is low, the material exhibits stress stiffening, but when the applied deviator stress is closer to the deviator stress at failure, the material exhibit stress softening. All these observations are consistent with those obtained by Lam (1982), Stolle et al. (2005), and Guo et. al. (2006), who found that, even though the resilient modulus of granular base/subbase aggregates from various sources was not affected substantially by the deviator stress, an increase in the deviator stress level may either increase or decrease the resilient modulus depending on the properties of the specific material. Similar observations can be found in the LTPP data report. 
It should be noted that strain hardening of $M_{r}$ (i.e., an increase of $M_{r}$ with deviator stress) is rarely observed for sand and clay, whose stiffness (both the elastic modulus and shear modulus) tends to decrease with an increase in shear strain that depends on the applied shear stress. When the shear stress-induced shear strain is at level of $0.0001 \%$, the material can be considered as linear elastic, which corresponds to the maximum shear modulus. The modulus decreases with the strain amplitude when the strain level is larger than $0.001 \%$. Experimental evidence can be found in Santucci de Magistris et al. (1999), Tatsuoka (2000), and Kweon and Kim (2000); among others.

The resilient Poisson's ratio also depends on the applied stresses and strain levels. When the confining pressure increases, the lateral deformation is restrained and the resilient Poisson's ratio decreases. Higher deviator stress may induce large lateral deformation partially due to dilation, which results in increased resilient Poisson's ratio. Hicks (1970), Brown and Hyde (1975) and Kolisoja (1997) all reported the similar findings under CCP test conditions.

\subsubsection{Stress path}

As mentioned in the previous section, due to the unavailability of proper equipment and testing capabilities, most of the resilient modulus tests are limited to CCP triaxial tests. The important effect of stress path and major principal stress rotation experienced in situ can therefore not be fully simulated. Research on the influence of stress path on the resilient behaviour of granular materials is very limited.

According to Lekarp (1997), Allen and Thompson (1974) compared the results 
It should be noted that strain hardening of $M_{r}$ (i.e., an increase of $M_{r}$ with deviator stress) is rarely observed for sand and clay, whose stiffness (both the elastic modulus and shear modulus) tends to decrease with an increase in shear strain that depends on the applied shear stress. When the shear stress-induced shear strain is at level of $0.0001 \%$, the material can be considered as linear elastic, which corresponds to the maximum shear modulus. The modulus decreases with the strain amplitude when the strain level is larger than $0.001 \%$. Experimental evidence can be found in Santucci de Magistris et al. (1999), Tatsuoka (2000), and Kweon and Kim (2000); among others.

The resilient Poisson's ratio also depends on the applied stresses and strain levels. When the confining pressure increases, the lateral deformation is restrained and the resilient Poisson's ratio decreases. Higher deviator stress may induce large lateral deformation partially due to dilation, which results in increased resilient Poisson's ratio. Hicks (1970), Brown and Hyde (1975) and Kolisoja (1997) all reported the similar findings under $\mathrm{CCP}$ test conditions.

\subsubsection{Stress path}

As mentioned in the previous section, due to the unavailability of proper equipment and testing capabilities, most of the resilient modulus tests are limited to CCP triaxial tests. The important effect of stress path and major principal stress rotation experienced in situ can therefore not be fully simulated. Research on the influence of stress path on the resilient behaviour of granular materials is very limited.

According to Lekarp (1997), Allen and Thompson (1974) compared the results 
obtained from CCP and VCP (variable confining pressure) tests and found that when the bulk stress is smaller than a specific value, the resilient modulus from CCP tests is higher than that from VCP tests; when the bulk stress is larger than the specific value, the modulus from VCP tests is higher. The difference in resilient modulus between these 2 types of tests was found to vary with the stress level. Brown and Hyde (1975) suggested that stress paths (VCP and CCP) have no influence on the resilient modulus, provided that the confining pressure in $\mathrm{CCP}$ test is equal to the mean confining pressure in VCP test. These conclusions may be questionable as the test materials in those studies are considered as isotropic. In other words, these analysis and findings would be inaccurate if the resilient properties of granular materials are direction-dependent.

In contrast to the findings of Brown and Hyde (1975), Seyhan and Tutumluer (2005) found that the stress path has an influence on the resilient properties of crushed stone. More details are presented in the following section.

\subsection{ANISOTROPY}

In addition to stress dependency, the resilient properties of unbound granular materials are direction-dependent; i.e., the stiffness in the horizontal direction is different from that in the vertical direction. Since soils and granular materials have horizontal bedding, it is often assumed that the stiffness properties do not vary in horizontal directions.

The deformation properties of granular materials are inherently anisotropic and can also become more anisotropic at more anisotropic stress states. Anisotropy (inherent anisotropy and stress-induced anisotropy) is a fundamental aspect of resilient behaviour 
of granular materials that is often ignored. Before research on the cross-anisotropy in soils and granular materials is reviewed, the description of cross-anisotropic elasticity is introduced.

To describe the stress-strain relation of a cross-anisotropic elastic material, 5 parameters are needed. They are elastic modulus in the vertical direction $E_{v}$, elastic modulus in the horizontal direction $E_{h}$, shear modulus in the vertical plane $G_{v h}$, Poisson's ratio for the effect of vertical strain on horizontal strain $v_{v h}$, and Poisson's ratio for the effect of horizontal strain on horizontal strain at right angle $v_{h h}$. Within this study, the resilient moduli $M_{r}^{h}, M_{r}^{v}, G_{r}^{h h}$ (shear modulus in the horizontal plane, which can be obtained from $E_{h}$ and $v_{h h}$ ) and $G_{r}^{v h}$ are surrogates for elastic moduli $E_{h}, E_{v}, G_{h h}$, and $G_{v h}$ of Hooke's law.

Table 2.1 summarizes previous investigations on cross-anisotropy in soils and aggregates. It can be seen that the effect of anisotropy on unbound aggregates has not been investigated as much as that on sand and clay.

Hardin (1978) proposed that the elastic modulus $E_{i}=\frac{\partial \sigma_{i}}{\partial \varepsilon_{i}}$ for sands in any particular direction $i$ is a unique function of the normal stress $\sigma_{i}$ working in direction $i$, independent of the normal stresses acting in the other orthogonal directions, which was later extended to a general form (Hardin and Blandford 1989, Jiang et al. 1997, Hoque and Tatsuoka 1998, among others). The elastic modulus $E_{i}$ in any direction is assumed to follow a power law,

$$
E_{i}=C_{i} f(e)\left(\frac{\sigma_{i}}{p_{r}}\right)^{m}
$$


where $f(e)$ is a void ratio function indicating the effect of density, $p_{r}$ is the reference pressure ( $=1 \mathrm{kPa}), m$ and $C_{i}$ are regression constants, which depend on the stress history of the material.

Jiang et al. (1997) investigated small strain $(\sim 0.001 \%)$ deformation characteristics of well-graded sandy gravel using large square prismatic samples $(23 \times 23 \times 57 \mathrm{~cm})$. At each stress state, very small load/unload cycles of vertical stress $\sigma_{v}$, with strain increments less than $0.001 \%$, were first applied at constant confining pressure; then very small unload/load cycles of horizontal stress $\sigma_{h}$, with strain increments less than $0.001 \%$, were applied at constant vertical stress. To explore the inherent anisotropy and stress inducedanisotropy at very small strain, the cyclic triaxial tests were performed at various isotropic and anisotropic stress states, with ratios of $\sigma_{v} / \sigma_{h}$ ranging from 0.5 2.2. The assumption of $v_{v h}=v_{h h}$ was employed in the study to determine the horizontal elastic modulus. The test results showed that at isotropic and anisotropic stress states, $E_{v}^{o}$, where “o" denotes strains less than $0.001 \%$, is consistently higher than $E_{h}^{o}$. Moduli, $E_{v}^{o}$ and $E_{h}^{o}$ are unique functions of $\left(\sigma_{v}\right)^{m}$ and $\left(\sigma_{h}\right)^{m}$, respectively, where $m \cong 0.5$. Therefore, the ratio of $E_{v}^{o} / E_{h}^{o}$ increases in proportion to $\left(\sigma_{v} / \sigma_{h}\right)^{m}$, which indicates that the stress-induced anisotropy increases with an increase in $\sigma_{v} / \sigma_{h}$. They found that $E_{v}^{o} / E_{h}^{o} \cong 2$ at isotropic stress states. As a result, they concluded that the sample has similar inherent anisotropy for both elastic and plastic deformation. However, they reported that $E_{h}^{o}$ and $E_{v}^{o}$ deviate from the trendline and become smaller when $\sigma_{v} / \sigma_{h}>2.2$ or $\sigma_{v} / \sigma_{h}<0.5$. 
Table 2.1 Previous investigations on cross-anisotropy in soils.

\begin{tabular}{|c|c|c|c|c|c|c|c|}
\hline Reference & Material & Stress state & Apparatus & Test & Assumption & Strain level & $\begin{array}{c}E_{h} / E_{v} \text { at isotropic } \\
\text { stress state }\end{array}$ \\
\hline $\begin{array}{l}\text { Graham \& Houlsby } \\
(1983)\end{array}$ & $\begin{array}{l}\text { Lightly over } \\
\text { consolidated clay }\end{array}$ & Isotropic & Triaxial & Proportional loading & $\frac{E_{h}}{E_{v}}=\left(\frac{v_{h h}}{v_{v h}}\right)^{2}$ & $2 \sim 4 \%$ & 1.8 \\
\hline $\begin{array}{l}\text { Hoque et al. } \\
\text { (1996) }\end{array}$ & Sand & $\begin{array}{l}\text { Isotropic } \\
\text { Anisotropic }\end{array}$ & Triaxial RPL & $\begin{array}{l}\Delta \sigma_{v}>0 ; \Delta \sigma_{h}=0 \\
\Delta \sigma_{v}=0 ; \Delta \sigma_{h}>0\end{array}$ & $v_{v h}=v_{h h}$ & $\sim 0.002 \%$ & $0.8 \sim 1$ \\
\hline $\begin{array}{l}\text { Jiang et al. } \\
(1997)\end{array}$ & Gravel & $\begin{array}{l}\text { Isotropic } \\
\text { Anisotropic }\end{array}$ & Triaxial RPL & $\begin{array}{l}\Delta \sigma_{v}>0 ; \Delta \sigma_{h}=0 \\
\Delta \sigma_{v}=0 ; \Delta \sigma_{h}>0\end{array}$ & $v_{v h}=v_{h h}$ & $\sim 0.001 \%$ & 0.5 \\
\hline $\begin{array}{l}\text { Zdravkovic \& Jardine } \\
\text { (1997) }\end{array}$ & $\begin{array}{l}\text { Normal to over } \\
\text { cosolidated clay }\end{array}$ & $\begin{array}{l}\text { Isotropic } \\
\text { Anisotropic }\end{array}$ & $\mathrm{HCA}$ & Various combination & & $\sim 10 \%$ & - \\
\hline $\begin{array}{l}\text { Lings et al. } \\
(2000)\end{array}$ & $\begin{array}{l}\text { Over consolidated } \\
\text { clay }\end{array}$ & $\begin{array}{l}\text { Isotropic } \\
\text { Anisotropic }\end{array}$ & Triaxial with bender & $\begin{array}{l}\Delta \sigma_{v}>0 ; \Delta \sigma_{h}=0 \\
\Delta \sigma_{v}=0 ; \Delta \sigma_{h}>0\end{array}$ & & $\sim 0.0001 \%$ & $\sim 4$ \\
\hline $\begin{array}{l}\text { Kuwano } \\
(2000)\end{array}$ & Sand & $\begin{array}{l}\text { Isotropic } \\
\text { Anisotropic }\end{array}$ & Triaxial with bender & $\begin{array}{l}\Delta \sigma_{v}>0 ; \Delta \sigma_{h}=0 \\
\Delta \sigma_{v}=0 ; \Delta \sigma_{h}>0\end{array}$ & & $\sim 0.0008 \%$ & $\sim 0.56$ \\
\hline $\begin{array}{l}\text { Chaudaahary et.al } \\
\text { (2004) }\end{array}$ & Sand & $\begin{array}{l}\text { Isotropic } \\
\text { Anisotropic }\end{array}$ & $\begin{array}{c}\text { Triaxial with bender } \\
\text { HCA }\end{array}$ & $\begin{array}{c}\Delta \sigma_{v}>0 ; \Delta \sigma_{h}=0 \\
\Delta \sigma_{v}=0 ; \Delta \sigma_{h}>0 \\
\Delta \sigma_{z}>0 ; \Delta \sigma_{\theta}=0 ; \Delta \sigma_{z \theta}=0 \\
\Delta \sigma_{z}=0 ; \Delta \sigma_{\theta}>0 ; \Delta \sigma_{z \theta}=0 \\
\Delta \sigma_{z}=0 ; \Delta \sigma_{\theta}=0 ; \Delta \sigma_{z \theta}>0\end{array}$ & & $\sim 0.005 \%$ & $\sim 0.72$ \\
\hline $\begin{array}{l}\text { Seyhan.et.al (2005) } \\
\text { Tuturmluer \& Seyhan } \\
(1999,2000)\end{array}$ & Aggregate & Isotropic & Triaxial RPL & $\begin{array}{c}\Delta \sigma_{v}=\Delta \sigma_{h} / 4>0 \\
\Delta \sigma_{v}=\Delta \sigma_{h} / 2>0 \\
\Delta \sigma_{v}=\Delta \sigma_{h}>0 \\
\Delta \sigma_{v}=4 \Delta \sigma_{h} / 7>0 \\
\Delta \sigma_{v}=3 \Delta \sigma_{h} / 4>0\end{array}$ & $\frac{E_{h}}{E_{v}}=\left(\frac{v_{h h}}{v_{v h}}\right)^{2}$ & & $0.3 \sim 0.5$ \\
\hline $\begin{array}{l}\text { Adu-Osei et al.(2000) } \\
\text { Masad et al.(2004) } \\
\text { Kim et al. (2005) }\end{array}$ & $\begin{array}{l}\text { Limestone } \\
\text { Gravel }\end{array}$ & Anisotropic & Triaxial RPL & $\begin{array}{c}\Delta \sigma_{v}>0 ; \Delta \sigma_{h}=0 \\
\Delta \sigma_{v}=-2 \Delta \sigma_{h}>0 \\
\Delta \sigma_{v}=-\Delta \sigma_{h}>0\end{array}$ & $\begin{array}{c}\text { Elastic work } \\
\text { potential }\end{array}$ & & $0.41 \sim 0.64$ \\
\hline $\begin{array}{l}\text { AnhDan et al. } \\
(2006)\end{array}$ & Gravel & $\begin{array}{l}\text { Isotropic } \\
\text { Anisotropic }\end{array}$ & Triaxial RPL & $\begin{array}{l}\Delta \sigma_{v}>0 ; \Delta \sigma_{h}=0 \\
\Delta \sigma_{v}=0 ; \Delta \sigma_{h}>0\end{array}$ & & $\sim 0.001 \%$ & $\begin{array}{l}\text { Depending on } \\
\text { stress level }\end{array}$ \\
\hline
\end{tabular}


Similar results have been reported for poor-graded sands (Hoque and Tatsuoka 1998), an undisturbed gravel (Koseki et al. 1999), and a well-graded gravel with crushed sandstone inside (Kohata 1997).

AnhDan et al. (2006) extended the research conducted by Jiang et al. (1997) and Kohata et. al. (1997) and performed tests on the same material using a true triaxial apparatus. With the true triaixal apparatus, the elastic moduli at strain levels of $0.001 \%$ and $0.0003 \%$ in the vertical and horizontal directions, respectively, could be studied directly and no assumption of $v_{h h}$ was required. Some of the test results are similar to those reported by Jiang et al. (1997); i.e., $E_{h}^{o}$ and $E_{v}^{o}$ are unique functions of $\sigma_{h}$ and $\sigma_{v}$, respectively, and can be expressed as

$$
\begin{aligned}
& E_{v}^{o}=\frac{E_{v_{r}}^{o}}{f\left(e_{r}\right)} f(e)\left(\frac{\sigma_{v}}{p_{r}}\right)^{m_{v}} \\
& E_{h}^{o}=\frac{E_{h_{r}}^{o}}{f\left(e_{r}\right)} f(e)\left(\frac{\sigma_{h}}{p_{r}}\right)^{m_{h}}
\end{aligned}
$$

where $p_{r}$ and $e_{r}$ are the reference pressure $(=1 \mathrm{kPa})$ and void ratio, respectively. $E_{v_{r}}^{o}$, $E_{h_{r}}^{o}, m_{v}$, and $m_{h}$ are regression constants.

Similarly, vertical Poisson's ratios $v_{v h}$ and $v_{h v}$ were found to depend on the ratio of $\sigma_{v} / \sigma_{h}$, given as

$$
\begin{aligned}
& v_{v h}=v_{v h_{r}}\left(\frac{\sigma_{v}}{\sigma_{h}}\right)^{n_{v h}} \\
& v_{h v}=v_{h v_{r}}\left(\frac{\sigma_{v}}{\sigma_{h}}\right)^{n_{h v}}
\end{aligned}
$$

where $v_{v h_{r}}$ and $v_{h v_{r}}$ are the values of $v_{v h}$ and $v_{h v}$ when $\sigma_{v}=\sigma_{h}$, respectively. $n_{v h}$ and $n_{h v}$ are regression constants. $v_{v h}$ and $v_{h v}$ are constants at isotropic stress states. 
However, some findings were not consistent with relevant studies conducted by Jiang et al. (1997) and Kohata et. al. (1997). At isotropic stress states, when the stress is low, $E_{v}^{o}$ is greater than $E_{h}^{o}$. When stress is at a level of $400 \mathrm{kPa}$ and higher, $E_{v}^{o}$ and $E_{h}^{o}$ becomes nearly same. These findings indicate that the inherent anisotropy, quantified by $\left(1-E_{h}^{o} / E_{v}^{o}\right)$ at isotropic stress states, is not a constant, but changes with stresses. As the specimens were compacted vertically during preparation stage, at low stress levels during the test, the soil structure may have been more stable in the vertical direction than the horizontal direction; $E_{v}^{o}$ is in turn consistently higher than $E_{h}^{o}$. When the stresses increase, soil fabric rearranges and the contact normal tends to become co-axial with the major principal stress direction in response to the increasing stresses. As such, it is possible that the material becomes isotropic when the applied stresses reach a certain level or higher.

It should be noted that the previous research findings appear to be contradictory to that from the resilient modulus testing, where the vertical resilient modulus not only depends on $\sigma_{v}$ but also on $\sigma_{h}$. One must be aware that for the testing conditions of the studies reported in the previous paragraphs, the strain levels are approximately $0.001 \%$. When $E_{v}^{o}$ was investigated, the strain $\varepsilon_{h}$ is much smaller than $\varepsilon_{v}$ and can be considered negligible, which leads to $E_{v}^{o}$ being independent of $\sigma_{h}$, and vice versa. Therefore the conclusion may only be accepted conditionally for very small strain behaviour of coarse grained materials. For granular materials in pavement practice, the resilient deformation, normally develops within very small to intermediate strains, needs further investigation.

Several researchers have studied the anisotropic resilient behaviour of aggregates (Barksdale et al. 1989, Tuturmluer 1995, Tuturmluer and Seyhan 1999, 
2000, 2004, Karasehin and Dawson 2000, Adu-Osei et al. 2000, Masad et al. 2004, Kim et al. 2005).

Adu-Osei et al. (2000) developed a testing protocol using a triaxial set up to investigate the influence of stress level on the anisotropic resilient properties of crushed limestone and aggregates. Three types of stress paths, triaixial compression, triaxial shear and triaxial extension, as shown in Table 2.1, were applied to the sample. All tests were conducted at anisotropic stress states, where $\sigma_{v} / \sigma_{h}$ ranges from 1.6 2.4. For each stress path test, very small load/unload cycles were applied on the samples. The anisotropic resilient properties back-calculated from the tests are results from 3 different stress paths. The cross-anisotropic moduli are shown to increase with an increase in bulk stress, with the vertical resilient moduli being consistently higher than the horizontal moduli. It should be noted that the effect of stress path on the resilient response of aggregates could however not be identified. In addition, the cyclic load/unload used in the tests was small, being at lower bound of stresses typically experienced in a pavement. The resilient properties obtained from the tests could be considered to be more quasi-elastic.

Seyhan and Tuturmluer (2005) investigated the directional dependency of resilient modulus and Poisson's ratio of a selected crushed aggregate. They conducted 6 constant stress path tests using an advanced triaxial apparatus. Of all tests, 5 "identical" samples were tested for 5 different stress paths, with slope of stress path $k=\Delta q / \Delta p=\frac{\Delta \sigma_{v}-\Delta \sigma_{h}}{\Delta \sigma_{v}+2 \Delta \sigma_{h}}=1.5,0.75,0,-0.5$, and -1 , respectively. To minimize the effect of material property variations, a single sample was tested for the 5 stress paths (stress path sweep) in the sequence of $k=-1.0,-0.5,0,0.75$, and 1.5.

The cross-anisotropic resilient moduli computed from the 5 different stress path 
tests and stress path sweep test, generally increased as the applied bulk stress and vertical deviator stress increase. Higher vertical moduli than horizontal moduli and higher vertical Poisson's ratios than horizontal Poisson's ratios were obtained from all tests. The vertical Poisson's ratio decreased with an increase in the bulk stress. For both vertical and horizontal Poisson's ratios, stress paths having $k=-1.0$ and 0 , yielded the highest and lowest Poisson's ratios, respectively.

There are some discrepancies in the results between stress path tests and stress path sweep test. When compared to stress paths with positive $k$, stress paths with negative $k$ often yielded higher vertical moduli and lower horizontal moduli given constant bulk stress. However, this trend changed for stress path sweep test, where higher vertical moduli were obtained for stress paths with positive $k$ at low bulk stress levels $(0 \sim 400 \mathrm{kPa})$, and horizontal moduli from each stress path were very close. The effect of stress paths on the anisotropic properties was not thoroughly investigated in their study. Despite the discrepancy and limited numbers of tests, their test results show that the direction of stress path does have an influence on the anisotropic moduli, though the influence is not quite clear. The variation of stress path effect on the resilient moduli from the stress path tests and stress path sweep test need further investigation by taking into account the stress and strain level commonly encountered in granular base/subbase layers.

Practical engineering problems invariably involve the rotation of principal stress, as well as changes in the intermediate principal stress. It should be noted that even though the VCP test is superior to the CCP test in simulating the field loading conditions, the principal stresses during the VCP test are only in the vertical and horizontal directions. The overall picture of the field loading conditions is not fully 
reflected in VCP tests as the stress history associated with the rotation of principal stress cannot be reproduced. Consequently, the effect of principal stress rotation (shear stress and reversal) on the resilient response of aggregates cannot be explored. The hollow cylinder apparatus (HCA) provides an opportunity to look into the effect of stress path, particularly principal stress rotation on the anisotropic resilient behaviour of granular materials.

By far, the influence of principal stress rotation on the resilient properties is not fully understood. Chan (1990) demonstrated that resilient strains are unaffected by the rotation of principal stress. He also showed that the principal resilient strains coincide with those of the stresses. The findings are somewhat questionable as granular materials are highly nonlinear and anisotropic, and the principal stresses do not necessarily coincide with principal strains unless the principal stresses act along the material axes.

In addition to the effect of stress path, other aspects, such as the effect of the mineral type, particle shape, fines content on the anisotropy of resilient properties are not well understood. Owing to the limited knowledge of the behaviour of coarse granular materials subjecting to cyclic loading, more research on the anisotropic property of granular materials under general stress conditions is required.

\subsection{EQUATIONS FOR RESILIENT MODULUS AND POISSON'S RATIO}

\subsubsection{Resilient modulus as a function of stresses}

Various relations have been proposed to describe the dependency of the resilient modulus on stress variables. Most of those in the literature are empirical equations based on the analysis of experimental results with regression techniques. Inherent in 
these equations is the assumption that the resilient properties of granular materials are too complex to combine the theoretical principles of soil mechanics with simplicity required in engineering practice. The most appropriate and simple relations for realistic characterizing the granular materials have yet to be found.

Table 2.2 presents some of the equations found in the literature, along with their test conditions and limitations. Among these nonlinear equations, the $k-\theta$ model, Uzan equation and AASHTO equation are the most popular in engineering practice.

Hicks (1971) recommended the use of a simple relation between resilient modulus and bulk stress, commonly known as the $k-\theta$ model, given by Eq. (2.10) in Table 2.2. Some researchers (Brown and Pappin, 1985, Uzan, 1985) have pointed out that this model has serious limitations leading to inaccurate results in pavement analysis, as it is unable to discriminate the effect of confining pressure and deviator stress on resilient modulus. Furthermore, it fails to recognize the effects of dilation and accumulation of permanent shear strains on a material's stiffness, in other words, the model may be insufficient for describing the effect of deviator stress for some subgrade soils or granular materials that have a high percentage of fines.

Realizing the effect of deviator stress on resilient modulus, Uzan $(1985,1992)$ improved the $k-\theta$ model by including the effect of deviator stress. The Uzan equation, as described by Eqs. (2.22) and (2.23) in Table 2.2, also known as the universal constitutive equation, is widely used. It generally yields better results than the $k-\theta$ model and fits the LTPP $M_{r}$ test data fairly well. To avoid the unrealistic prediction of $M_{r}$ at low deviator stress when the regression coefficient $k_{3}$ is negative, the Guide for Mechanistic - Empirical Design of New and Rehabilitated Pavement Structures for the Design of Flexible Pavement Structures, recommends a modified version of the 
Uzan equation, as presented in Eq. (2.24) in Table 2.2. However, it should be noted that these equations are only capable of modelling either increase or decrease of $M_{r}$ as the deviator stress increases, which depends on whether the coefficient $k_{3}$ is positive or negative. The popularity of these equations rests in their ability to fit the experimental data well. The main shortcomings are that they do not have theoretical basis and the success to fitting data is primarily for conventional compression loading paths.

Van Niewerk (2002) examined the influence of deviator stress on the resilient modulus by performing a series of CCP and VCP triaxial tests. The resilient modulus and Poisson's ratio were determined by solving the following equations

$$
\begin{gathered}
\Delta \varepsilon_{v}=\frac{1}{M_{r}}\left(\Delta \sigma_{v}-2 v \Delta \sigma_{h}\right) \\
\Delta \varepsilon_{h}=\frac{1}{M_{r}}\left(\Delta \sigma_{h}(1-v)-v \Delta \sigma_{v}\right)
\end{gathered}
$$

He noticed that for coarse grained materials, at lower ratios of $\sigma_{d} / \sigma_{d, f}$ (deviator stress at failure), the materials displayed stress stiffening behaviour; but at stress levels closer to failure, the materials exhibited stress softening behaviour. Therefore, he suggested that triaxial testing should be extended to more severe loading conditions to measure the important stress-softening behaviour. To model the behaviour observed in the tests, a parabolic resilient modulus equation was proposed to account for the stiffening effect of confining pressure, and initial stiffening and subsequent softening effect of deviator stress, given by Eq. (2.28) in Table 2.2. However, the parabolic resilient equation does not reflect the influence of stress paths or differentiate the resilient properties in different directions as the results from VCP tests are based on the assumption that the tested material is isotropic. 
Table 2.2 Resilient modulus equations

\begin{tabular}{|c|c|c|c|c|c|c|c|}
\hline Reference & Equation & & Test & Isotropic & Anisotropic & Limitation/Drawback & $\begin{array}{l}\text { Symbols } \\
k_{1} \sim k_{9}: \text { regression constants } \\
\theta=\sigma_{1}+\sigma_{2}+\sigma_{3}: \text { bulk stress } \\
q=\sigma_{v}-\sigma_{h} \\
p=\left(\sigma_{1}+2 \sigma_{3}\right) / 3 \\
J_{2 D}=3 / 2\left(\tau_{o c t}\right)^{2}: 2^{\text {nd }} \text { invariant of the } \\
\text { deviator stress } \\
v: \text { constant Poisson's ratio } \\
J_{2}=\sigma_{1} \sigma_{2}+\sigma_{1} \sigma_{3}+\sigma_{2} \sigma_{3} \\
\tau_{o c t}=1 / 3 \\
\sqrt{\left(\sigma_{1}-\sigma_{2}\right)^{2}+\left(\sigma_{2}-\sigma_{3}\right)^{2}+\left(\sigma_{1}-\sigma_{3}\right)^{2}} \\
J_{2}: 2^{\text {nd }} \text { invariant of stress } \\
\tau_{o c t}: \text { octahedral shear stress } \\
p_{r}: \text { reference pressure. } \\
p_{a}: \text { atmouspheric pressure }(=100 \mathrm{kPa}) \\
\varepsilon_{v o l}: \text { volumetric strain } \\
\varepsilon_{q}=2 / 3\left(\varepsilon_{v}-\varepsilon_{h}\right): \text { shear strains } \\
\alpha=(1-c) K_{1} / G_{1}, \\
c, d, h, K_{1} \text { and } G_{1}: \text { material parameters } \\
p_{1}, q_{1}, p_{2}, q_{2}: \text { stress state } 1 \text { and } 2 \\
l=\sqrt{\Delta p^{2}+\Delta q^{2}}: \text { length of stress path } \\
E_{t}: \text { tangent stiffness } \\
E_{t_{r}}: \text { tangent stiffness at reference } \\
\text { stress } \\
\sigma_{d, f}: \text { deviator stress at failure }\end{array}$ \\
\hline $\begin{array}{l}\text { Dunlop (1963) } \\
\text { Monismith (1967) }\end{array}$ & $M_{r}=k_{1}\left(\sigma_{3} / p_{r}\right)^{k 2}$ & $(2.9)$ & $\mathrm{CCP}$ & $\checkmark$ & & Neglecting the effect of deviator stress & \multirow{14}{*}{$\begin{array}{l}k_{1} \sim k_{9}: \text { regression constants } \\
\theta=\sigma_{1}+\sigma_{2}+\sigma_{3}: \text { bulk stress } \\
q=\sigma_{v}-\sigma_{h} \\
p=\left(\sigma_{1}+2 \sigma_{3}\right) / 3 \\
J_{2 D}=3 / 2\left(\tau_{o c t}\right)^{2}: 2^{\text {nd }} \text { invariant of the } \\
\text { deviator stress } \\
v: \text { constant Poisson's ratio } \\
J_{2}=\sigma_{1} \sigma_{2}+\sigma_{1} \sigma_{3}+\sigma_{2} \sigma_{3} \\
\tau_{o c t}=1 / 3 \\
\sqrt{\left(\sigma_{1}-\sigma_{2}\right)^{2}+\left(\sigma_{2}-\sigma_{3}\right)^{2}+\left(\sigma_{1}-\sigma_{3}\right)^{2}} \\
J_{2}: 2^{\text {nd }} \text { invariant of stress } \\
\tau_{o c t}: \text { octahedral shear stress } \\
p_{r}: \text { reference pressure. } \\
p_{a}: \text { atmouspheric pressure (=100kPa) } \\
\varepsilon_{v o l}: \text { volumetric strain } \\
\varepsilon_{q}=2 / 3\left(\varepsilon_{v}-\varepsilon_{h}\right): \text { shear strains } \\
\alpha=(1-c) K_{1} / G_{1}, \\
c, d, h, K_{1} \text { and } G_{1}: \text { material parameters } \\
p_{1}, q_{1}, p_{2}, q_{2}: \text { stress state } 1 \text { and } 2 \\
l=\sqrt{\Delta p^{2}+\Delta q^{2}}: \text { length of stress path } \\
E_{t}: \text { tangent stiffness } \\
E_{t r}: \text { tangent stiffness at reference } \\
\text { stress } \\
\sigma_{d, f}: \text { deviator stress at failure }\end{array}$} \\
\hline Hicks (1971) & $M_{r}=k_{1}(\theta)^{k 2}$ & $(2.10)$ & $\mathrm{CCP}$ & $\checkmark$ & & Neglecting the effect of deviator stress & \\
\hline Boyce (1980) & $\begin{array}{l}K_{r}=p / \varepsilon_{v o l} G_{r}=\sigma_{d} / 3 \varepsilon_{q} \\
\varepsilon_{v o l}=p^{c}\left(1-\beta \sigma_{d}^{2} / p^{2}\right) / K_{1} \\
\varepsilon_{q}=p^{c}(q / p) /\left(3 G_{1}\right)\end{array}$ & $\begin{array}{l}(2.11) \\
(2.12) \\
(2.13)\end{array}$ & $\mathrm{CCP}$ & $\checkmark$ & & $\begin{array}{l}\text { Poor prediction of strains, particularly } \\
\text { volumetric strains (Sweere, 1990) }\end{array}$ & \\
\hline $\begin{array}{l}\text { Pappin \& Brown } \\
(1980,1985)\end{array}$ & $\begin{array}{l}\varepsilon_{v}=p\left(p / p_{r}\right)^{c-1}\left(1-\alpha \sigma_{d}^{2} / p^{2}\right) / K_{1} \\
\varepsilon_{q}=\left(p / p_{r}\right)^{d-1} q /\left(3 G_{1}\right) \\
\Delta \varepsilon_{q}=\left(\left(p_{2} / p_{r}\right)^{d}\left(q_{2} / p_{2}\right)-\right. \\
\left.\left(p_{1} / p_{r}\right)^{d}\left(q_{1} / p_{1}\right)\right) /\left(3 G_{1}\right) l^{h}\end{array}$ & $\begin{array}{l}(2.14) \\
(2.15) \\
(2.16)\end{array}$ & $\mathrm{CCP}$ & $\checkmark$ & & $\begin{array}{l}\text { Neglecting shear stress-induced by } \\
\text { dilatancy in volume }\end{array}$ & \\
\hline Johnson et al. (1986) & $M_{r}=k_{1}\left(J_{2} / \tau_{o c t}\right)^{k_{2}}$ & $(2.17)$ & $\mathrm{CCP}$ & $\checkmark$ & & $\begin{array}{l}\text { Predict unrealistic resilient moduli at } \\
\text { low deviator stresses }\end{array}$ & \\
\hline Lade \& Nelson (1987) & $\begin{array}{l}M_{r}=k_{1} p_{a}\left(\left(\theta / p_{a}\right)^{2}+6 J_{2 D}(1+\right. \\
\left.v) /(1-2 v) / p_{a}^{2}\right)^{k_{2}}\end{array}$ & $(2.18)$ & $\mathrm{CCP}$ & $\checkmark$ & & $\begin{array}{l}\text { Poisson's ratio not a constant, } \\
\text { unrealistic }\end{array}$ & \\
\hline Brown et al. (1987) & $G_{r}=K_{1} \sigma_{d}\left(p / \sigma_{d}\right)^{k_{2}}$ & $(2.19)$ & $\mathrm{CCP}$ & $\checkmark$ & & $\begin{array}{l}\text { Predicting unrealistic resilient moduli at } \\
\text { low deviator stresses }\end{array}$ & \\
\hline $\begin{array}{l}\text { Brown (1975) } \\
\text { Tam \& Brown (1988) }\end{array}$ & $M_{r}=K_{1}\left(p / \sigma_{d}\right)^{k_{2}}$ & $(2.20)$ & $\mathrm{CCP}$ & $\checkmark$ & & $\begin{array}{l}\text { Predicting unrealistic resilient moduli at } \\
\text { low deviator stresses }\end{array}$ & \\
\hline $\begin{array}{l}\text { Pezo (1993) } \\
\text { Garg \& Thompson (1997) }\end{array}$ & $M_{r}=k_{1}{\sigma_{d}}^{k_{2}} \sigma_{3}^{k_{3}}$ & $(2.21)$ & $\mathrm{CCP}$ & $\checkmark$ & & $\begin{array}{l}\text { Predicting unrealistic resilient moduli at } \\
\text { low deviator stresses }\end{array}$ & \\
\hline Uzan $(1985,1992)$ & $\begin{array}{l}M_{r}=k_{1}\left(\theta / p_{a}\right)^{k_{2}}\left(\sigma_{d} / p_{a}\right)^{k_{3}} \\
M_{r}=k_{1}\left(\theta / p_{a}\right)^{k_{2}}\left(\tau_{o c t} / p_{a}\right)^{k_{3}}\end{array}$ & $\begin{array}{l}(2.22) \\
(2.23)\end{array}$ & $\mathrm{CCP}$ & $\checkmark$ & & $\begin{array}{l}\text { Only describing either an increase } \\
\text { /decrease of } M_{r} \text { with increasing } \sigma_{d} \text { or } \\
\tau_{o c t}, \text { neglecting effect of stress path }\end{array}$ & \\
\hline AASHTO (2002) & $M_{r}=k_{1}\left(\theta / p_{a}\right)^{k_{2}}\left(\tau_{o c t} / p_{a}+1\right)^{k_{3}}$ & $(2.24)$ & $\mathrm{CCP}$ & $\checkmark$ & & Same as above & \\
\hline $\begin{array}{l}\text { Tuturmluer \& Seyhan } \\
(1999,2000,2003) \\
\text { Karasehin \& Dawson (2000) } \\
\text { Adu-Osei et al. (2000) } \\
\end{array}$ & $\begin{array}{l}M_{r}^{h}=k_{1}\left(\theta / p_{a}\right)^{k_{2}}\left(\sigma_{d} / p_{a}\right)^{k_{3}} \\
M_{r}^{v}=k_{4}\left(\theta / p_{a}\right)^{k_{5}}\left(\sigma_{d} / p_{a}\right)^{k_{6}} \\
G_{r}^{v h}=k_{7}\left(\theta / p_{a}\right)^{k_{a}}\left(\sigma_{d} / p_{a}\right)^{k_{9}}\end{array}$ & $\begin{array}{l}(2.25) \\
(2.26) \\
(2.27)\end{array}$ & $\begin{array}{c}\mathrm{CCP} \\
\& \\
\mathrm{VCP}\end{array}$ & & $\checkmark$ & Neglecting effect of stress path & \\
\hline Van Niekerk (2002) & $\begin{array}{l}M_{r}=k_{1}\left(\sigma_{3} / p_{r}\right)^{k_{2}}\left(k_{3}\left(\sigma_{3} / \sigma_{d, f}\right)^{2}+\right. \\
\left.k_{4}\left(\sigma_{3} / \sigma_{d, f}\right)+1\right)\end{array}$ & $(2.28)$ & VCP & $\checkmark$ & & Neglecting effect of stress path & \\
\hline Konrad (2006) & $E_{t}=E_{t_{r}}\left(\sigma_{v} / p_{r}\right)^{k_{1}}$ & $(2.29)$ & VCP & $\checkmark$ & & $\begin{array}{l}\text { Predicting unrealistic resilient moduli at } \\
\text { high } \sigma_{v} \text {, neglecting effect of } \sigma_{h}\end{array}$ & \\
\hline
\end{tabular}


CEN (Comité Européen de Normalisation, 2004) also uses Eqs. (2.30) and (2.31) to determine the resilient properties from VCP resilient modulus tests, which may result in lower resilient modulus and Poisson's ratio than obtained from CCP tests. However, the resilient moduli and Poisson's ratios from VCP tests computed by using Eqs. (2.30) and (2.31) are not real vertical moduli or real vertical Poisson's ratios as it assume the material is isotropic.

Apart from resilient modulus, an alternative way of modelling the resilient response is to use resilient bulk modulus and resilient shear modulus. To obtain the resilient bulk modulus and resilient shear modulus, Boyce (1980) proposed an empirical relation to predict the recoverable volumetric and shear strains. Boyce's model, which assumes that the material is isotropic, unfortunately gives poor prediction of strains, particularly volumetric strains (Sweere, 1990). Boyce's model was subsequently modified by other researchers (Pappin \& Brown 1980, 1985, Elhannani 1991, Jouve and Elhannani 1993 as reported by Brown 1996 and Lekarp et al. 2000), with more parameters being introduced to give a better fit to the experimental data. Nevertheless, the determination of the parameters is not an easy task, and therefore has not been received well by design engineers, who prefer simple and reliable procedures.

Pappin and Brown $(1980,1985)$ extended the model developed by Boyce to a contour model, in which the stress dependency of resilient deformation is included in the formulation. The recoverable shear and volumetric strain depend on the length of stress path applied, and are expressed as contours in the $p-q$ stress space, shown in Fig. 2.4. The contour model is however an improvement over Boyce's model, as it is capable of 
predicting resilient test results much better. The limitation of the contour model lies in it not being able to describe the shear-induced dilatancy of some granular materials.
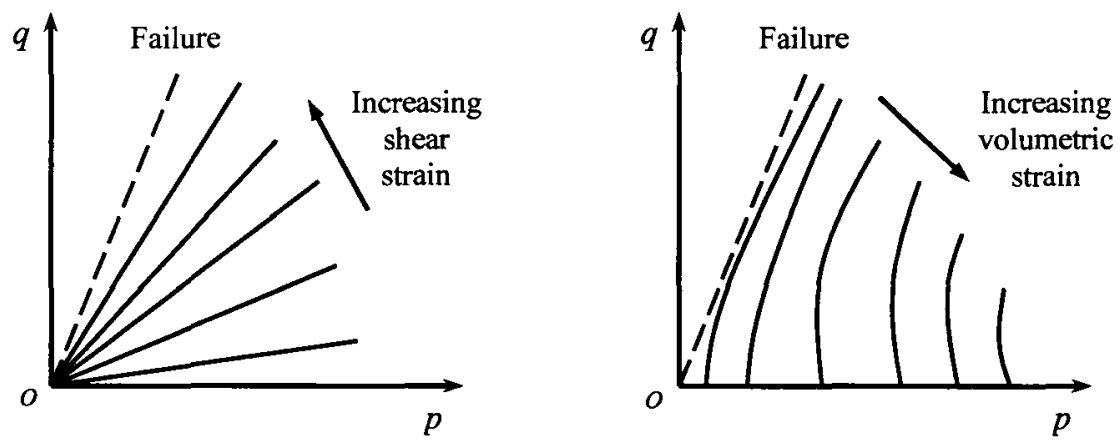

Fig. 2.4 Contour model in $p-q$ space (Brown and Pappin, 1985)

With regard to modelling cross-anisotropic resilient behaviour of unbound aggregates, Tuturmluer and Seyhan (1999, 2000, 2003), Karasehin and Dawson (2000) and Adu-Osei et al. (2000) assumed that the resilient modulus in different directions obeys the Uzan equation and thus, can be represented by the same stress-dependent formulation with different values of coefficients.

All the equations listed in Table 2.2 are based on the results of CCP or VCP triaxial tests. Owing to the limitation of the stress levels and stress paths applied in typical tests, the equations mentioned above are unable to fully describe the resilient response of unbound aggregates under more general stress conditions. More research must be conducted on the influence of stress path on the anisotropic resilient behaviour of granular materials. 


\subsubsection{Poisson's ratio as a function of stresses}

Compared to resilient modulus, very little research has been carried out on the resilient Poisson's ratio. In engineering practice, Poisson's ratio is often assumed to be constant for simplicity. Previous studies however have shown that Poisson's ratio of granular materials is stress- and direction-dependent. Under CCP triaxial test conditions, the Poisson's ratio $v$, strictly speaking the vertical Poisson's ratio $v_{v h}$, clearly depends on the confining pressure and the applied axial deviator stress. It decreases as the confining pressure increases, and increases as $\sigma_{v} / \sigma_{h}$ increases. Hicks and Monismith (1971), proposed a third-degree polynomial expression to model the variation of Poisson's ratio with stresses, given as

$$
v=a_{0}+a_{1}\left(\frac{\sigma_{v}}{\sigma_{h}}\right)+a_{2}\left(\frac{\sigma_{v}}{\sigma_{h}}\right)^{2}+a_{3}\left(\frac{\sigma_{v}}{\sigma_{h}}\right)^{3}
$$

where $a_{0}, a_{1}, a_{2}$, and $a_{3}$ are regression constants.

To account for the observed behaviour in the VCP and CCP tests, Van Niekerk (2002) developed two Poisson's ratio equations

$$
\begin{gathered}
v=A_{0}\left(\frac{\sigma_{d}}{\sigma_{d, f}}\right)^{A_{1}} \sigma_{h}^{A_{2}} \\
v=\left(B_{0}\left(\frac{\sigma_{d}}{\sigma_{d, f}}\right)+B_{1}\left(\frac{\sigma_{d}}{\sigma_{d, f}}\right)^{2}+B_{2}\left(\frac{\sigma_{d}}{\sigma_{d, f}}\right)^{3}\right) \sigma_{h}^{B_{3}}
\end{gathered}
$$

where $A_{0} \sim A_{2}, B_{0} \sim B_{3}$ are regression constants.

As mentioned previously, when Poisson's ratio is greater than 0.5 , the material exhibits dilatant behaviour, which for a truly linear elastic isotropic material is 
impossible. As shown in Figs. 2.3(b) and (c), the material is subjected to volume increase due to the shear stress. The larger the ratio of $\sigma_{v} / \sigma_{h}$ or $\sigma_{d} / \sigma_{d, f}$, the bigger the Poisson's ratio.

\subsection{SUMMARY}

The basic features of the resilient behaviour of granular materials are nonlinear, stress- and direction-dependent; i.e., anisotropy. The anisotropic resilient properties of granular materials are influenced by many factors, including physical properties and stress conditions. With regard to physical properties, the moisture and density are the most important, while the impact of stress level and stress/strain path on the resilient responses is most important of all the influential factors.

The influence of stress level on the resilient properties has long been recognized. A number of empirical resilient modulus equations have been proposed in terms of various stress variables. The $k-\theta$ model, Uzan equation and AASHTO equation are now widely used in practice. In general, resilient modulus and Poisson's ratio, depend on the level of confining pressure and deviator stress.

Research has shown that resilient modulus and Poisson's ratio are directiondependent. Granular materials in pavement generally display higher vertical resilient modulus and Poisson's ratio than horizontal ones, attributed to vertical compaction and horizontal bedding plane.

The potential importance of stress path and principal stress rotation on the resilient response has been noted, but due to the limitation of equipment and complexity of 
materials, how these facets influence the resilient behaviour is unclear and needs to be investigated. In view of the state-of-the-art for pavement analysis, proper characterization of the material properties is crucial for realistic analysis of pavement performance. 


\section{TESTING MATERIALS, EQUIPMENT, DESIGN AND PROCEDURES}

\subsection{INTRODUCTION}

This chapter presents the experimental program including the test matrix, the equipment for the resilient modulus test, material, and procedures for sample preparation and testing. The performance of the testing system and repeatability of the results are also discussed.

\subsection{EXPERIMENTAL DESIGN METHODOLOGY}

\subsubsection{Paths of loading and unloading}

Under a moving wheel load, a representative element in a granular base layer is subjected to stress changes that involve both the magnitude and the direction of the principal stresses. Based on the analysis of the Federal Aviation Administration's (FAA) National Airport Pavement Test Facility (NAPTF) full-scale test data, Tutumluer and Kim (2003) obtained stress paths (loading and unloading curves) induced by a single pass of a slow moving dual wheels configuration. Figure 3.1 shows the stress paths in $p-q$ space of the subgrade based on measured horizontal and vertical stresses at points TO \#1 and TO \#2. As $\Delta \sigma_{v}=\Delta p+\frac{2}{3} \Delta q, \Delta \sigma_{h}=\Delta p-\frac{1}{3} \Delta q$, it can be seen that when the wheel approaches, the soil element is loaded with an increase in stresses. Initially, $\Delta \sigma_{h}$ is higher than $\Delta \sigma_{v}$. As the wheel continues to approach, $\Delta \sigma_{v}$ gradually approaches $\Delta \sigma_{h}$. Afterwards, $\Delta \sigma_{v}$ becomes greater than $\Delta \sigma_{h}$ (It should be noted that $\Delta \sigma_{h}$ may decrease whereas $\Delta \sigma_{v}$ increases during this stage). When the wheel passes the centerline of the element, it is 
unloaded with a decrease in stresses.

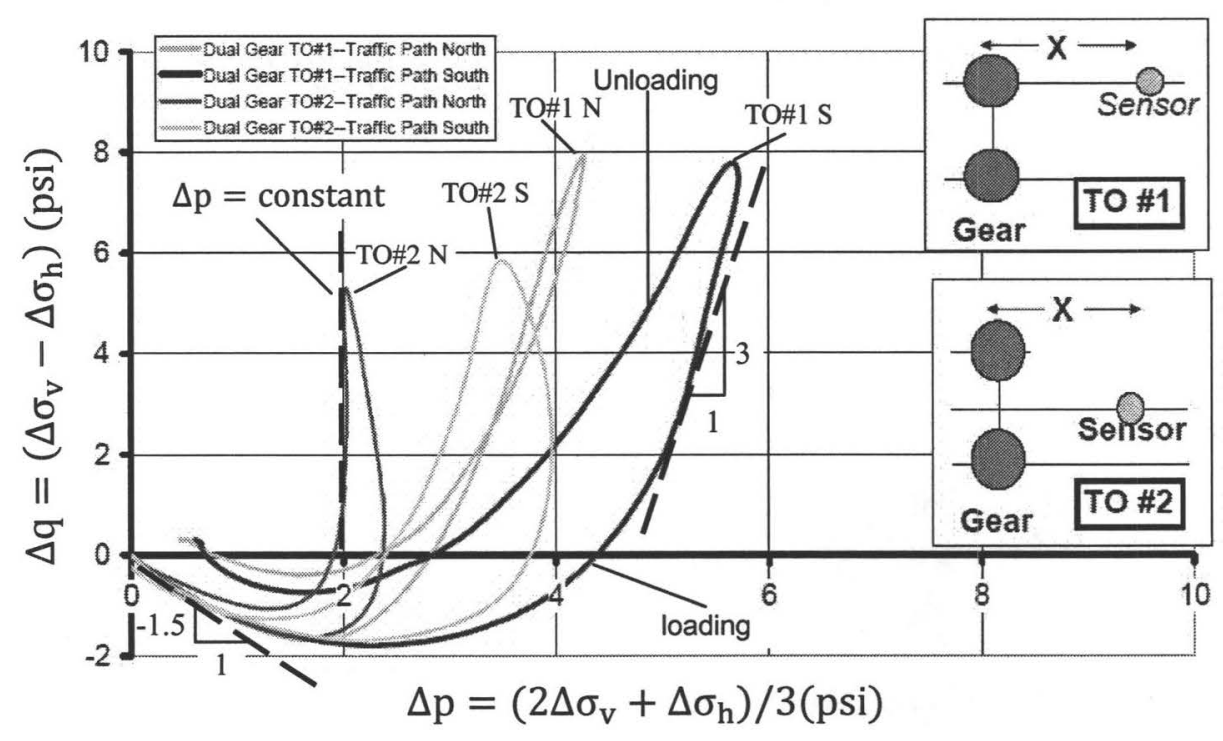

Fig. 3.1 Actual rolling wheel stress paths from the National Airport Pavement Tests Facility (Tutumluer and Kim, 2003)

Referring to the principal stress rotation induced by moving wheels illustrated in Fig. 1.1 and the stress variations in Fig. 3.1, it is observed that stress changes within a pavement layer are quite complex as a vehicle passes. Such a loading history is not adequately reflected in the standard resilient modulus test. Moreover, CCP test has limited capability to evaluate the anisotropic resilient properties. In order to understand the influence of stress path on measured resilient properties of an anisotropic material, multiple stress path tests are considered in this study. Figure 3.2 schematically shows the loading paths used for the resilient modulus tests, which are commonly encountered within a pavement layer. Table 3.1 summarizes the stress increments along these stress paths corresponding to different initial stress conditions. 


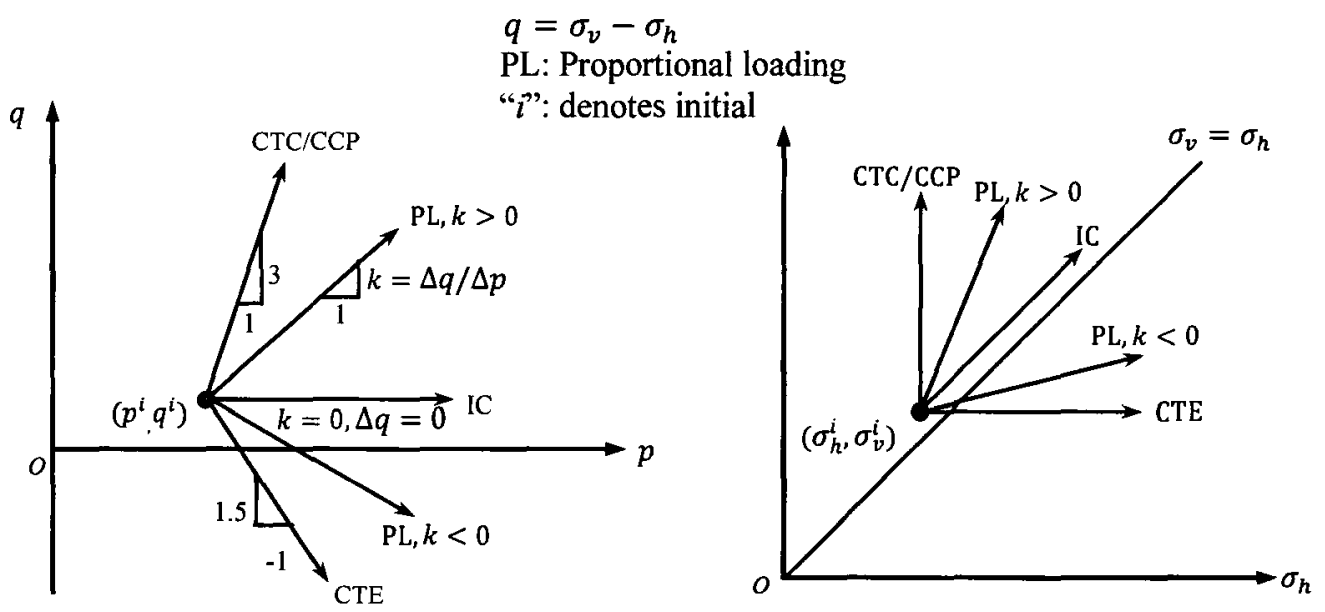

Fig. 3.2 Schematic of stress paths in testing depicted in stress spaces

Table 3.1 Explanation of stress paths

\begin{tabular}{llcc}
\hline Stress Path $^{*}$ & \multicolumn{2}{|c}{ Stress increments } \\
\hline IC $\quad$ Isotropic compression & $k=0$ & $\Delta \sigma_{v}=\Delta \sigma_{h}>0$ \\
CTC Conventional triaxial compression & $k=3$ & $\Delta \sigma_{v}>0 ; \Delta \sigma_{h}=0$ \\
CTE Conventional triaxial extension & $k=-1.5$ & $\Delta \sigma_{v}=0 ; \Delta \sigma_{h}>0$ \\
PL $\quad$ Proportional loading & $k$ & $\Delta \sigma_{v}=(2 k+3) /(3-k) \Delta \sigma_{h}$ \\
\hline
\end{tabular}

* Stress path in testing is followed the definition by Desai (2001)

\subsubsection{Initial stress state}

The resilient modulus of a granular soil is known to be not constant but a state dependent quantity. In general, the initial stress state can be used as an indicator for the stressdependency of resilient modulus. The in-situ initial vertical stress $\sigma_{v}$ at a point of interest in a pavement layer can be estimated as the overburden stress, with the initial horizontal stress $\sigma_{h}$ being determined as

$$
\sigma_{h}=K_{o} \sigma_{v}
$$


where $K_{o}$ is the coefficient of earth pressure at rest. In this study, $K_{\text {ini }}$ is used to replace $K_{o}$ to quantify the ratio of horizontal stress to vertical stress at an initial stress state.

Within the framework of AASHTO T 307-99 Resilient Modulus Testing Protocol, the hydrostatic pressure corresponding to $K_{i n i}=1$ is applied on the specimen prior to the resilient modulus test. Obviously, this stress state is different from real stress conditions in the field. During pavement construction, granular soils are usually well compacted, which induces substantial volume densification and strong fabric (or internal structure). Significant horizontal residual stresses may develop in the granular layers as a result of compaction. As observed in both laboratory and field tests, the large lateral earth pressures may even be higher than the vertical stress (i.e., $K_{i n i}>1$ ). For example, Uzan(1985) reported lateral residual stresses of $15 \mathrm{kPa}$ and $40 \mathrm{kPa}$ in compacted cohessionless and cohesive materials, respectively, behind retaining walls and bridge abutments; Stewart et al. (1985) measured horizontal stresses up to $35 \mathrm{kPa}$ in railway blasts; Selig (1987) carried out a tank model test of 2 layer (sand/clay) soil systems and found that the horizontal compressive stresses which developed at the bottom of the sand layer, were as high as $10 \mathrm{kPa}$ and $20 \mathrm{kPa}$ after 20 and 50 applications of loading cycles, respectively; Rydén (2004) reported a horizontal residual stress of $32 \mathrm{kPa}$ due to compaction in the unbound granular base layer from surface wave testing. These observations are consistent with the following well-accepted equation (Schmidt 1966) for overconsolidated soils

$$
K_{o}=(1-\sin \phi) O C R^{\sin \phi}
$$

where $\phi$ and $O C R$ are friction angle and overconsolidation ratio, respectively. 
In order to understand the influence of initial stress states on the measured resilient properties of an anisotropic material, both insotropic and anisotropic initial stress states are considered in this study.

\subsection{TESTING PROGRAM}

To investigate the resilient behaviour of granular materials with special reference to anisotropy, the following two test series, which accommodate the effect of initial stress state and stress path, were conducted:

- Test Series A: This series of tests was carried out to study the influence of the applied stresses along various stress paths $(-1.5 \leq k \leq 3.0)$. The specimens were subjected to isotropic consolidations and stress paths with various combination of confining pressures and deviator stresses.

- Test Series B: This series of tests was performed to explore the effect of $K_{\text {ini }}$ on the resilient properties. The specimens were subjected to different anisotropic consolidations, with $K_{\text {ini }}$ in the range of 0.5 to 2 , and stress paths ( $k=3.0$ and -1.5 ) at a selected initial lateral confinement of $50 \mathrm{kPa}$.

The stress paths and initial stresses adopted for the tests are presented in Figs. 3.2 and 3.3, respectively. Details of test program are given by Tables AI.1 and AI.2 in Appendix I. When examining the effect of stress path, the same specimen was used to study resilient behaviour for different stress paths provided that the permanent strain did not exceed $1 \%$. This allowed reducing the effect due to the variability between specimens. 


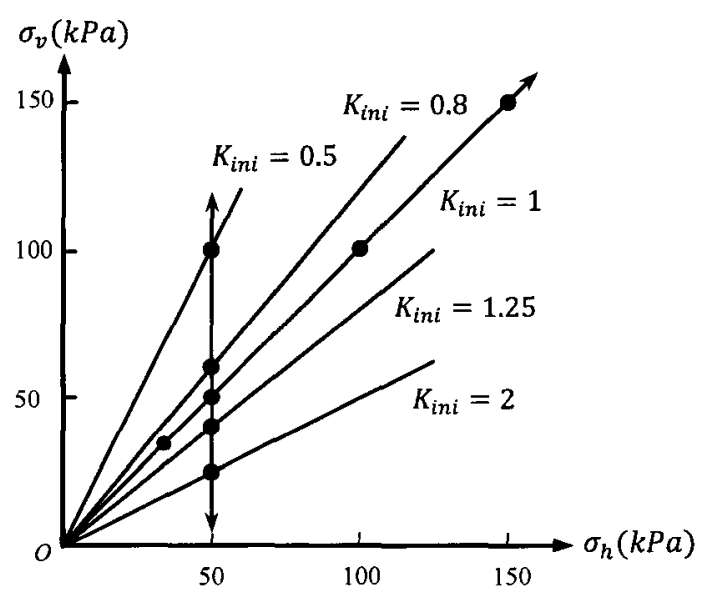

Fig. 3.3 Initial stresses employed

\subsection{DESCRIPTION OF TESTING EQUIPMENT}

The test device used for this study is a Wykeham Farrance Dynamic Hollow Cylinder Apparatus. The apparatus is a digitally controlled, servo-pneumatic (closed loop) system, which is capable of performing isotropic and anisotropic consolidation, standard triaxial tests, as well as HCA and resilient modulus tests. It can also perform $K_{o}$ consolidations, stress/strain path control tests, cyclic/dynamic stress path tests and other user defined tests.

Figure 3.4 shows a picture of Wykeham Farrance Dynamic Hollow Cylinder Apparatus. The axial load is applied though a pneumatic controlled actuator. The cell pressure and back pressure are controlled through pneumatic valves. Axial load, axial displacement, radial displacement, pore water pressure, volume change and cell pressure, are measured by different types of transducers, including load cell, Linear Variable Differential Transducer (LVDT), pore pressure transducer, and volume change transducer. An Integrated Multi-Axis Control System (IMACS) is linked to a laptop to 
provide critical control, timing and data acquisition for tests and the transducers. Detailed information about the testing equipment is presented in Tables AI.3 of Appendix I.

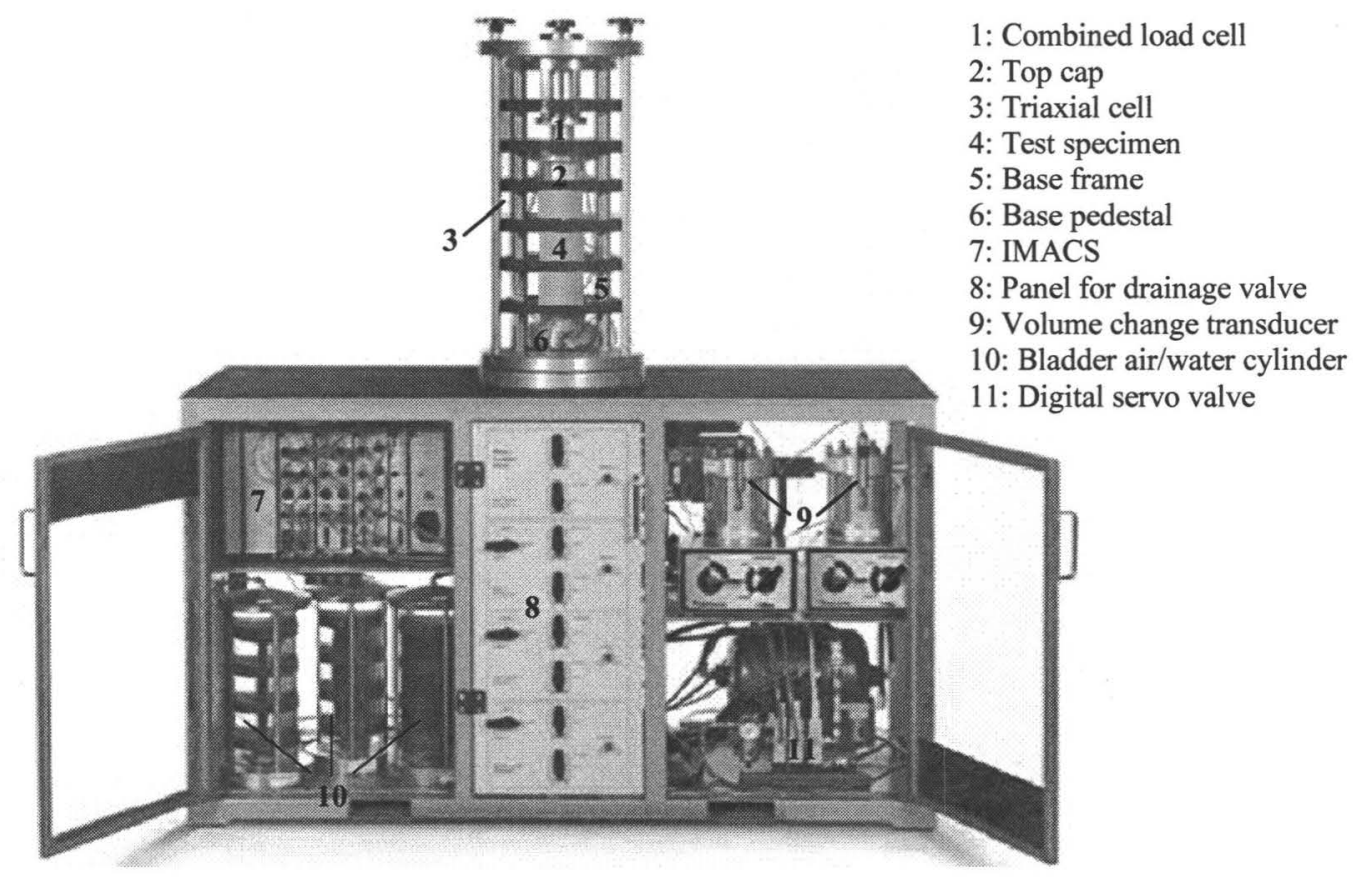

Fig. 3.4 Wykeham Farrance Dynamic Hollow Cylinder Apparatus

\subsection{MATERIALS}

A coarse sand with angular particles derived from crushed limestone was selected for the stress path tests. Figure 3.5 shows a photo of typical particles of the material, and Fig. 3.6 presents the particle size distribution curve obtained from sieve analysis. Following the standard test methods ASTM D4254-00 and D4253-00, the maximum and minimum void ratios were measured as 1.201 and 0.520 , respectively. The specific gravity of the material determined according to the standard test method ASTM C127-07 is 2.75. Before testing, a specimen was compacted to $95 \%$ of maximum dry density, with the moisture content being controlled at $5 \%$. 


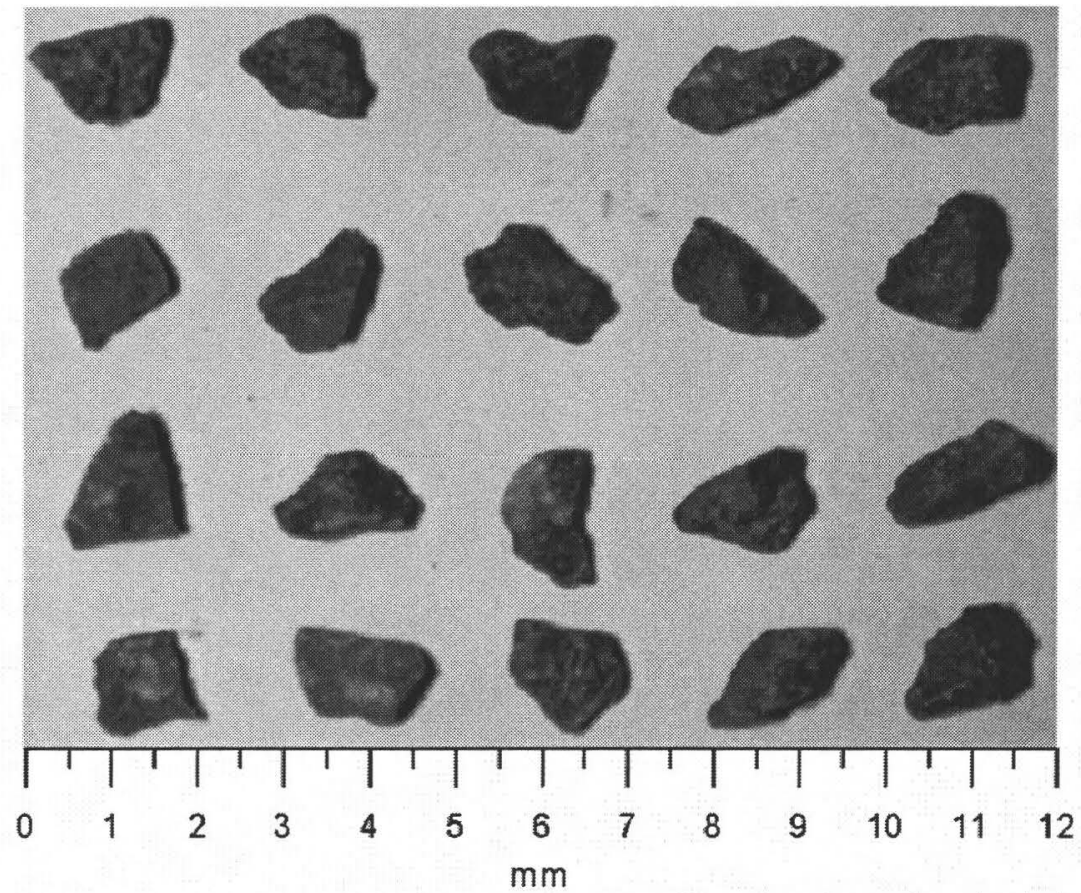

Fig. 3.5 Particles of crushed limestone

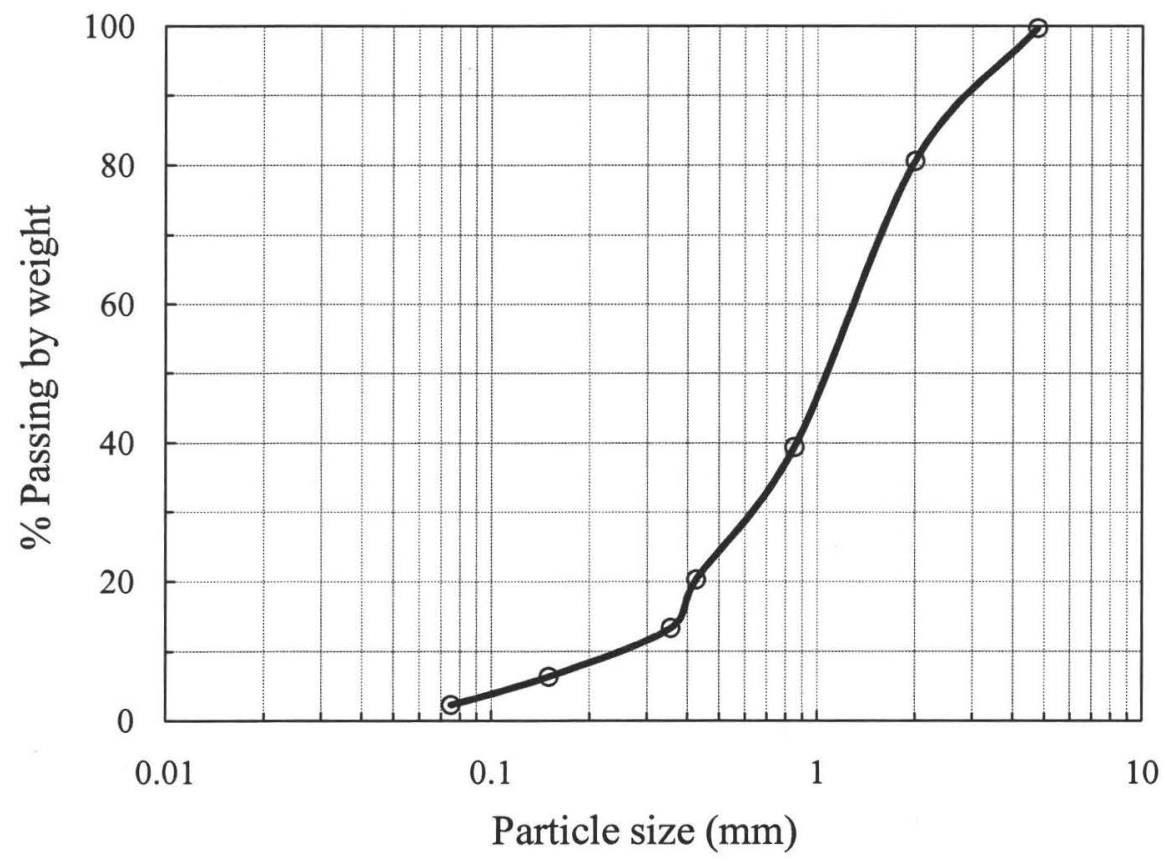

Fig. 3.6 Grain size distribution 


\subsection{TEST PROCEDURES}

\subsubsection{Sample preparation}

Cylindrical specimens, $100 \mathrm{~mm}$ in diameter and $200 \mathrm{~mm}$ high were fabricated in a split mould for stress path testing. A $0.6 \mathrm{~mm}$ membrane was first attached to the pedestal and secured with an O-ring. Thereafter the mould was placed around the pedestal and the membrane was stretched around the mould. The material was placed in the mould in 4 equal layers, with each layer being compacted uniformly to a nominal thickness of $50 \mathrm{~mm}$ by a steel tamper of $50 \mathrm{~mm}$ diameter. Before the next layer of loose material was poured into the mould, the surface of the sample was scratched to avoid layering at interfaces between adjacent layers. The specimen was compacted with the density of each layer being strictly controlled. This allowed consistency to be established between the specimens. After completion of the compaction, the top cap was placed on the sample and the membrane was sealed around the perimeter of the top cap.

\subsubsection{Specimen setup}

Before dismantling the split mould, a vacuum of $10 \mathrm{kPa}$ was applied on the specimen so that it could stand by itself. The axial actuator was brought up as needed to fix the specimen on the base frame, and the chamber was assembled and filled with de-aired water. As shown in Fig. 3.7, two LVDTs were attached to the specimen at mid-height to measure the vertical and radial deformation. The limitation of the equipment lies in that only one LVDT is used for displacement measurement in each direction, while the normal practice is to use two LVDTs. The strain level $(0,0.5 \%)$ in this research is far less than 
$5 \%$ and the nominal height to diameter ratio is 2 , so the effect of end friction may be considered to be very small (Bishop and Henkel 1957, Barden and Khayatt 1966). The end friction was minimized using a highly polished platen with centrally located polyester filter cloth. Before conditioning a specimen, a cell pressure of $100 \mathrm{kPa}$ was applied on the specimen and the vacuum was released at the same time. Thereafter, the cell pressure was maintained and 750 repetitions of a load equivalent to a maximum axial cyclic stress of $100 \mathrm{kPa}$ were applied for conditioning. The load pulse was haversine shaped with a frequency of $0.2 \mathrm{~Hz}$. During the cyclic loading stage, the drainage valve remained open to minimize the potential for the build-up of excess pore pressure.

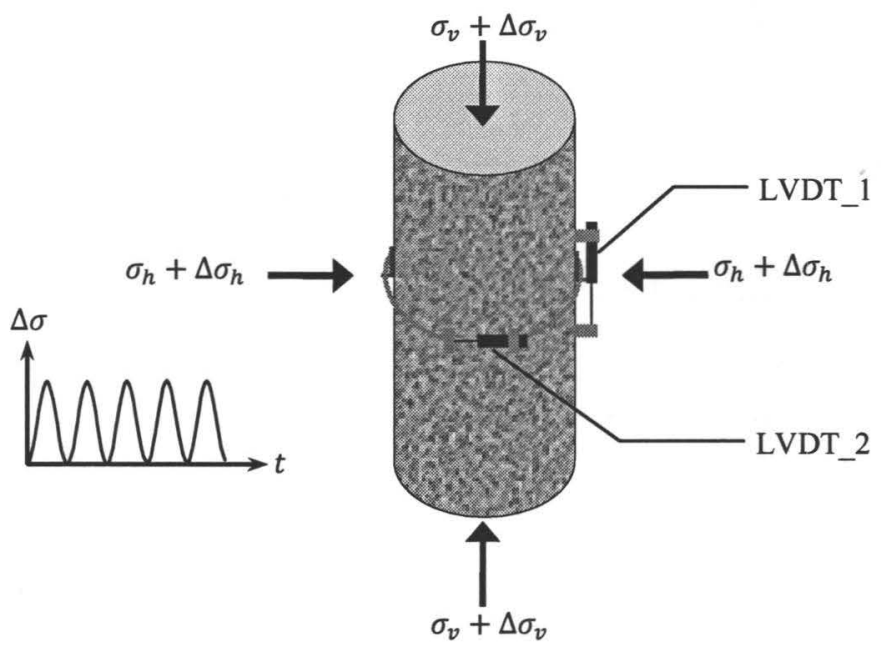

Fig. 3.7 Representation of the specimen

\subsubsection{Consolidation}

Following the conditioning stage, isotropic or anisotropic consolidation was performed according to the selected value of $K_{i n i}$. During consolidation, the vertical and horizontal stresses were brought to desired values by adjusting the cell pressure and axial load 
gradually. The readings of each transducer measuring the axial and radial deformation, cell pressure, and load cell throughout the consolidation were recorded to calculate the deformation of the test specimen.

\subsubsection{Cyclic loading tests}

After completion of consolidation, the specimen was subjected to stress paths listed in Tables AI.1 and AI.2 of Appendix I. In each test, 50 cycles of haversine-shaped load pulses was applied to the specimen. As the impact of loading frequency on the resilient properties is of little significance and the interest of this research is not on the frequency, but on the stress path, a frequency of $0.2 \mathrm{~Hz}$ was used throughout all stages of stress path tests for a good and stable performance of the test apparatus. The readings of LVDTs, cell pressure, and load cell for each load cycle were recorded at $0.1 \mathrm{~s}$ interval.

\subsection{PERFORMANCE AND REPEATABILITY}

To verify the repeatability of the specimen preparation method and stress path tests, preliminary tests were conducted on two specimens. The density and moisture content of the specimens were controlled at $1845 \mathrm{~kg} / \mathrm{m}^{3}$ and $5 \%$, respectively. Following the test procedure, the specimens were preconditioned and consolidated at a cell pressure of 100 $\mathrm{kPa}$. Thereafter cyclic deviator stresses were applied with controlled $\Delta \sigma_{h}$ and $\Delta \sigma_{v}$. A typical example of raw data on the measured stresses and deformation is presented in Fig. AI.1 of Appendix I, it can be seen that the noise in the data is very small. The responses for each sample from both CCP and VCP tests are shown in Fig. 3.8. One observes that 
the repeatability following the designed test procedure is satisfactory since the response curves are very close when the 2 specimens were subjected to same stress paths.
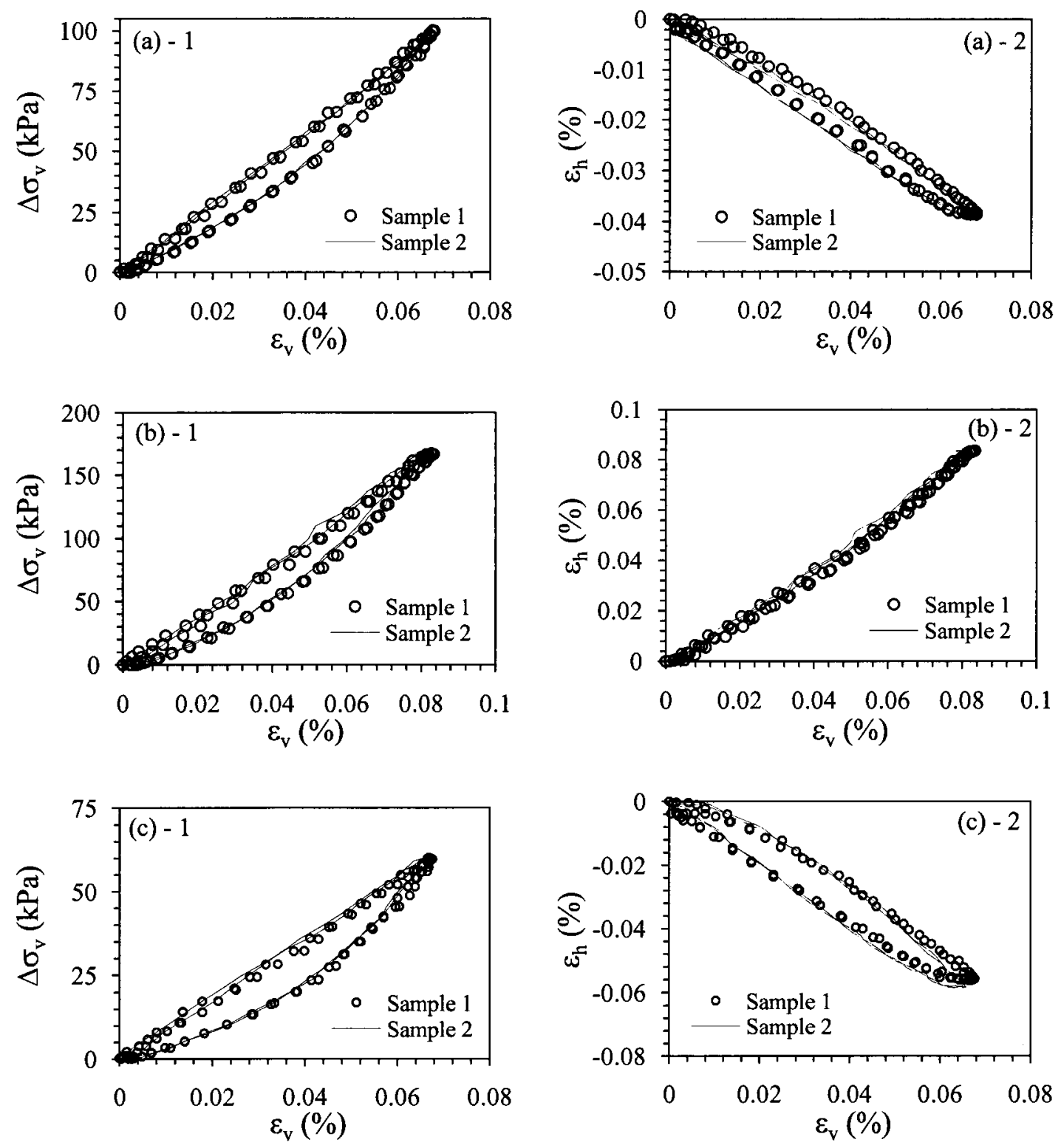

Fig. 3.8 Repeatability of stress path tests (Continued)

(a) $\sigma_{c}=100 \mathrm{kPa} \Delta \sigma_{h}=0 \Delta \sigma_{v}=100 \mathrm{kPa}$

(b) $\sigma_{c}=100 \mathrm{kPa} \Delta \sigma_{h}=67 \mathrm{kPa} \Delta \sigma_{v}=167 \mathrm{kPa}$

(c) $\sigma_{c}=30 \mathrm{kPa} \Delta \sigma_{h}=0 \Delta \sigma_{v}=60 \mathrm{kPa}$ 

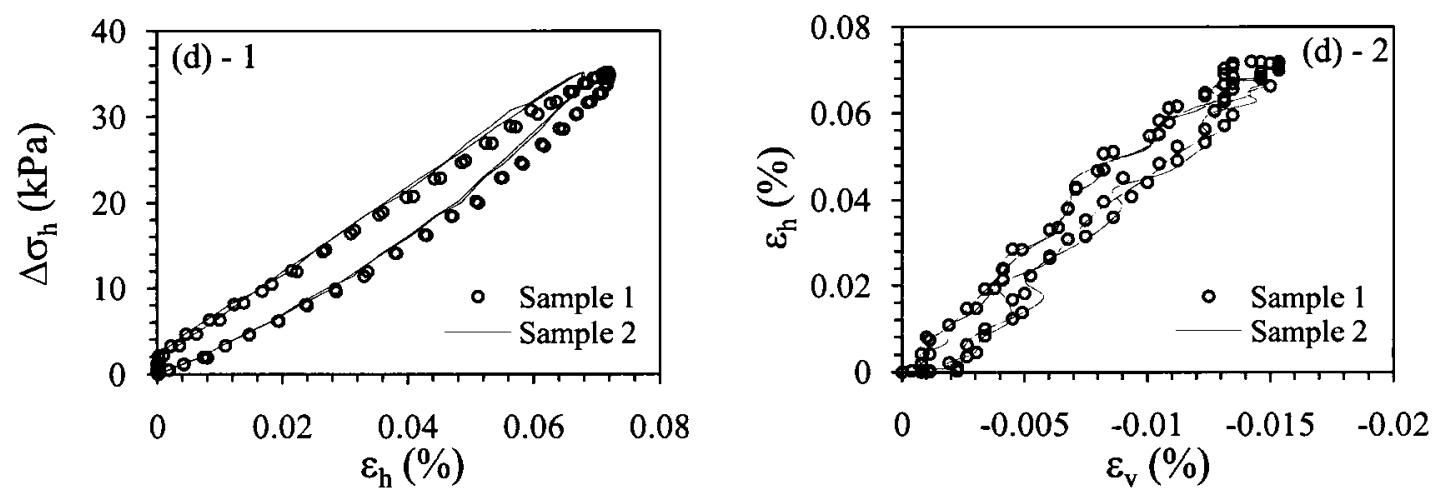

Fig. 3.8 Repeatability of stress path tests

(d) $\sigma_{c}=50 \mathrm{kPa} \Delta \sigma_{h}=35 \mathrm{kPa} \Delta \sigma_{v}=0$

\subsection{SUMMARY}

This chapter presented the methodology for the experimental investigation. Based on the measured stress paths in pavement layers obtained from full-scale tests, an extensive testing program was implemented to investigate the resilient behaviour of a granular material for various stress paths. The influences of initial stresses, residual horizontal stresses induced by compaction during construction, applied stress levels and stress paths on the resilient response of granular base/subbase were all considered in the test program.

In addition, the test apparatus, the specimen preparation method and test procedures were described in detail. Control tests were conducted to evaluate the repeatability and reproducibility of the proposed stress path tests. The results of the control tests demonstrate the repeatability and reliability of the proposed testing procedures are satisfactory. 


\section{RESILIENT BEHAVIOUR FOR ISOTROPIC CONSOLIDATION}

\subsection{INTRODUCTION}

This chapter explores through a series of $\mathrm{CCP}$ and VCP triaxial tests the resilient behaviour of granular materials that has been subjected to initial isotropic consolidation. The resilient responses of the material along different stress paths are described, which include typical stress-strain loops and resilient stress-strain relations. Resilient moduli and Poisson's ratios are presented, along with the directional dependency and the stress/strain path dependency. Both the inherent and stress-induced stiffness anisotropy are characterized in terms of stress state. New equations are developed to characterize the resilient modulus and Poisson's ratio of cross-anisotropic granular materials for general stress/strain conditions.

As stated in Chapter 3, each stress path test consisted of 4 levels of cell pressure $\left(\sigma_{c}=30,50,100\right.$ and $\left.150 \mathrm{kPa}\right)$. For each designated confining pressure, 4-5 levels of repeated stresses were applied on the specimen to obtain the corresponding stress-strain responses and the resilient properties. For each stress path test, the stresses and the corresponding deformations of the last 5 cycles were averaged to obtain the representative stresses and resilient strains that were used to calculate the resilient moduli and Poisson's ratios of each stage. 


\subsection{STRESS-STRAIN RESPONSES}

\subsubsection{Stresses and strain loops}

\section{(a) Isotropic material}

For an ideal linear elastic isotropic material, the stress-strain relations are expressed as

$$
\begin{gathered}
\varepsilon_{v}=\frac{\Delta \sigma_{v}-2 v \Delta \sigma_{h}}{E} \\
\varepsilon_{h}=\frac{(1-v) \Delta \sigma_{h}-v \Delta \sigma_{v}}{E}
\end{gathered}
$$

Scrutiny of Eq. (4.2) reveals that the coefficient associated with $\Delta \sigma_{h}$ is not $\frac{1}{E}$, but rather $\frac{1-v}{E}$. This is due to stress contributions coming from 2 principal stress directions. For this reason, the horizontal stiffness appears to be greater than the vertical stiffness when interpreting the experimental results; i.e. $\frac{E}{1-v}>E$.

The shear modulus $G$ and bulk modulus $K$, can be related to $E$ and $v$ via

$$
\begin{gathered}
G=\frac{E}{2(1+v)} \\
K=\frac{E}{3(1-2 v)}
\end{gathered}
$$

where Eq. (4.3) also holds for cross-anisotropic material, with $G, E$ and $v$ being replaced with $G_{h h}, E_{h}$ and $v_{h h}$, respectively.

For CCP tests, in which $\Delta \sigma_{h}=0$, the relations of $\varepsilon_{v} v s \varepsilon_{h}$ and $\varepsilon_{v o l} v s \gamma_{o c t}$ (volume $v s$ octahedral shear strain) are given via

$$
\frac{\varepsilon_{v}}{\varepsilon_{h}}=-\frac{1}{v}
$$




$$
\begin{gathered}
\varepsilon_{v o l}=3 \varepsilon_{o c t}=\varepsilon_{1}+\varepsilon_{2}+\varepsilon_{3}=\frac{\Delta \sigma_{v}}{E}(1-2 v) \\
\gamma_{o c t}=\frac{2 \sqrt{2}}{3}\left|\varepsilon_{1}-\varepsilon_{3}\right|=\frac{2 \sqrt{2}}{3} \frac{\Delta \sigma_{v}}{E}|1+v| \\
\frac{\varepsilon_{v o l}}{\gamma_{o c t}}=\frac{3 \sqrt{2}}{4} \frac{1-2 v}{|(1+v)|}
\end{gathered}
$$

For other stress paths with $\Delta \sigma_{h}>0$ and $-1.5 \leq k<3.0$, let $\iota=\frac{\Delta \sigma_{v}}{\Delta \sigma_{h}}=\frac{2 k+3}{3-k}$, the relations of $\varepsilon_{h} v s \varepsilon_{v}$ and $\varepsilon_{v o l} v s \gamma_{o c t}$ are given by

$$
\begin{gathered}
\frac{\varepsilon_{v}}{\varepsilon_{h}}=\frac{\iota-2 v}{1-v-\imath v} \\
\varepsilon_{v o l}=3 \varepsilon_{o c t}=\varepsilon_{1}+\varepsilon_{2}+\varepsilon_{3}=\frac{\Delta \sigma_{h}}{E}(\iota+2)(1-2 v) \\
\gamma_{o c t}=\frac{2 \sqrt{2}}{3}\left(\varepsilon_{1}-\varepsilon_{3}\right)=\frac{2 \sqrt{2}}{3} \frac{\Delta \sigma_{h}}{E}|(\iota-1)(1+v)| \\
\text { For } \iota \neq 1 \quad \frac{\varepsilon_{v o l}}{\gamma_{o c t}}=\frac{3 \sqrt{2}}{4}\left|\frac{(\iota+2)(1-2 v)}{(\iota-1)(1+v)}\right|=\frac{9 \sqrt{2}}{4} \frac{1-2 v}{|k(1+v)|}
\end{gathered}
$$

Equations (4.1) through (4.4) indicate that the stress-strain relations for an ideal isotropic material are linear and depend on $E, v$ and/or $\iota$ (or $k$ ). It can be seen from Figs. 4.1(a) and (b) that the trends of variations of $\sigma_{h} v s \varepsilon_{h}$ and $\sigma_{v} v s \varepsilon_{v}$ are similar and stresspath dependent, whereas the relations of $p$ vs $\varepsilon_{v o l}$ and $\tau_{o c t} v s \gamma_{o c t}$ are independent of stress path, see Figs. 4.1(c) and (d).

Equations (4.5), (4.8), (4.9) and (4.12) reveal that the relations of $\varepsilon_{v} v s \varepsilon_{h}$ and $\varepsilon_{o c t} v s \gamma_{o c t}$ are linear and depend on stress path, as demonstrated in Figs. 4.1(e) and (f). An important observation for a linear isotropic material is that the major principal strain is 
coaxial with the major principal stress. For the IC loading path, $\iota=1, \varepsilon_{h}=\varepsilon_{v}$, therefore $\gamma_{o c t}=0$, which implies that there is no shear strain coupled with volume change.

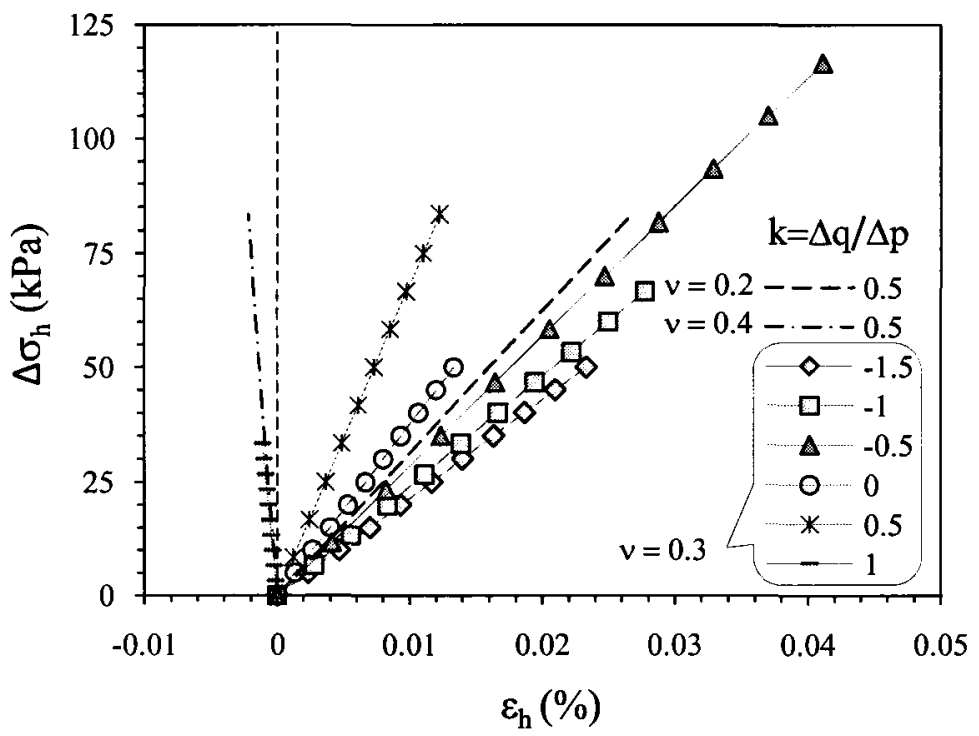

(a)

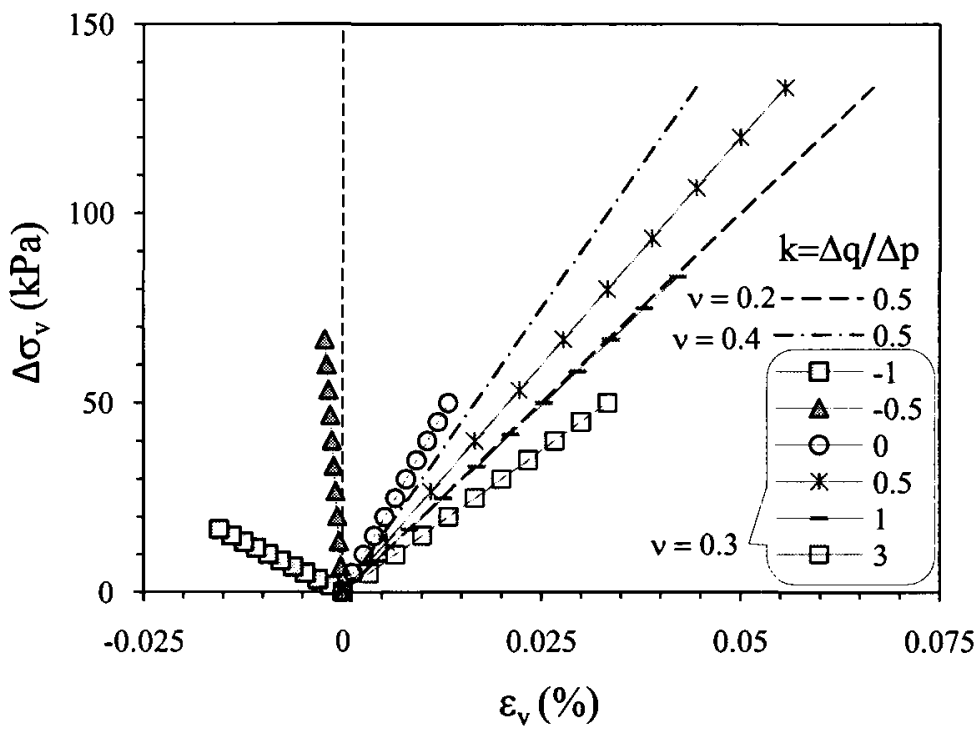

(b)

Fig. 4.1 Predicted responses of a linear isotropic material (continued)

$$
\begin{gathered}
E=150 \mathrm{MPa} \quad v=0.3 \\
\sigma_{d}=\sigma_{1}-\sigma_{3}=50 \mathrm{kPa} \quad \sigma_{c}=50 \mathrm{kPa}
\end{gathered}
$$




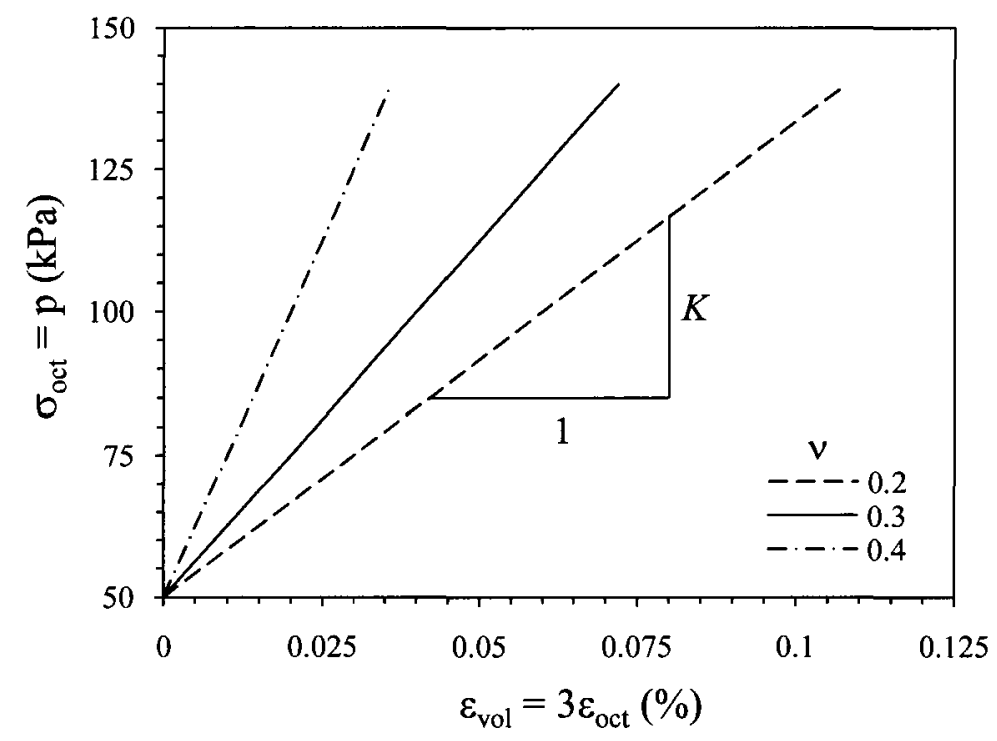

(c)

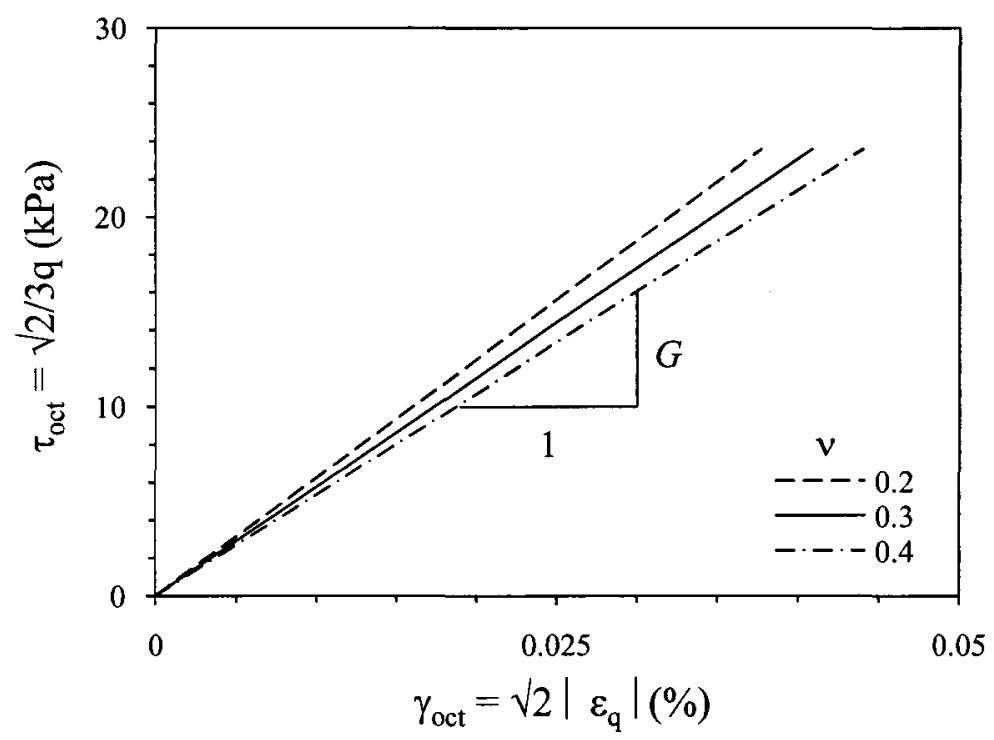

(d)

Fig. 4.1 Predicted responses of a linear isotropic material (continued)

$$
\begin{gathered}
E=150 \mathrm{MPa} \quad v=0.3 \\
\sigma_{d}=50 \mathrm{kPa} \quad \sigma_{c}=50 \mathrm{kPa}
\end{gathered}
$$




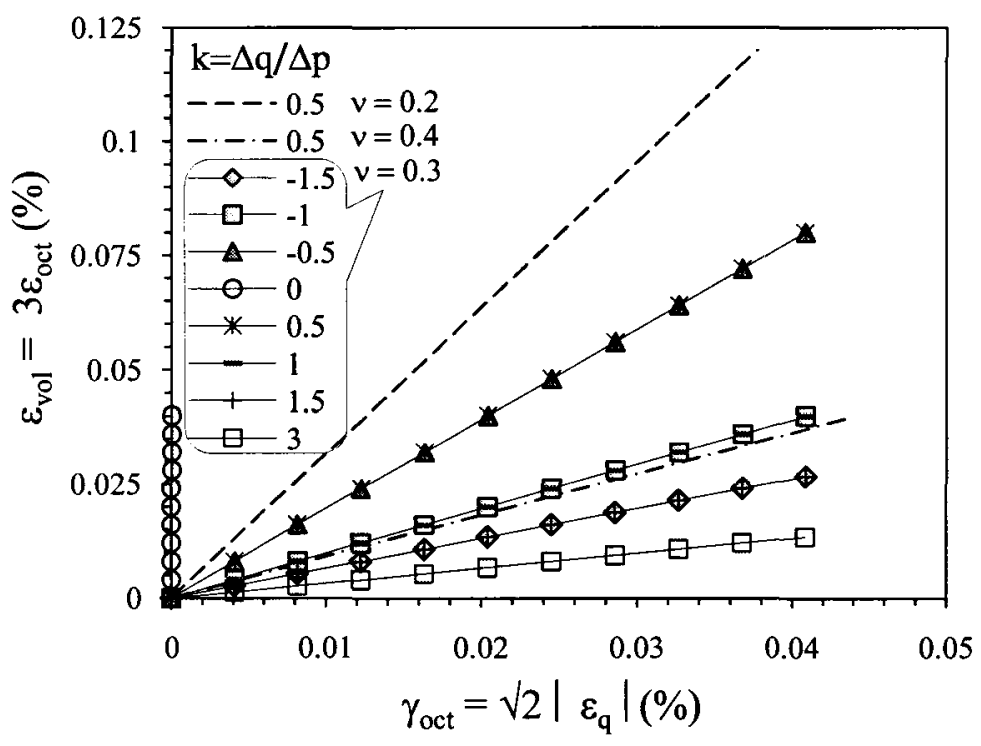

(e)

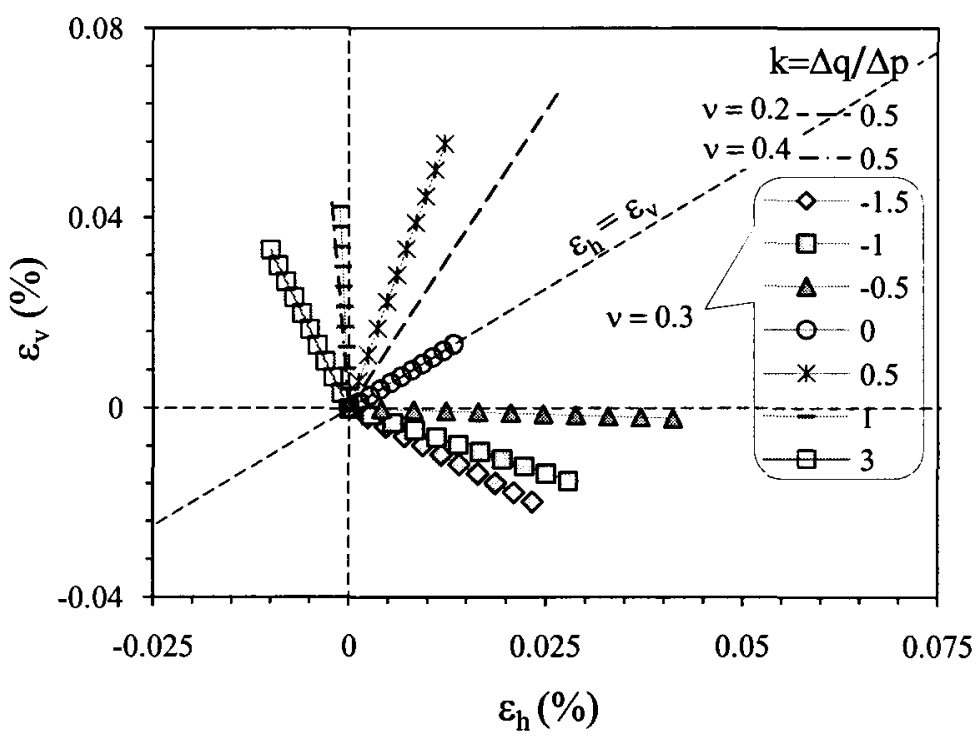

(f)

Fig. 4.1 Predicted responses of a linear isotropic material

$$
\begin{gathered}
E=150 \mathrm{MPa} \quad v=0.3 \\
\sigma_{d}=50 \mathrm{kPa} \quad \sigma_{c}=50 \mathrm{kPa}
\end{gathered}
$$


With respect to the nonlinear isotropic material, Eqs. (4.1) and (4.2) do not hold, but the equations for the stress-strain relations are similar and one must proceed incrementally to capture changes in stiffness due to stress sensitive moduli. For nonlinear isotropic materials subjected to the same stress paths and initial stress states, the overall trends of the stress-strain relations are similar to those of linear isotropic materials, but variations are nonlinear, as shown in Figs. 4.2(a) through (d), which summarize the responses of a nonlinear isotropic material, whose elastic modulus is assumed to be pressure-dependent. Based on this assumption, the relations of $p v s \varepsilon_{v o l}$ only depend on $v$ and $E$, and are independent of stress path. The $\tau_{o c t} v s \gamma_{o c t}$ relation is however not only depends on $v$ and $E$, but also on stress path, as seen in Figs. 4.2(c) and (d). When the values of $|k|$ are the same, the $\tau_{o c t} v s \gamma_{o c t}$ relations are identical given constant $v$. With respect to the trends of $\varepsilon_{v} v s \varepsilon_{h}$ and $\varepsilon_{v o l} v s \gamma_{o c t}$ given constant $v$ and $\iota$ (or $k$ ), they are identical to those of linear isotropic materials, respectively, as Eqs. (4.5), (4.8), (4.9) and (4.12) still hold for nonlinear isotropic materials. 


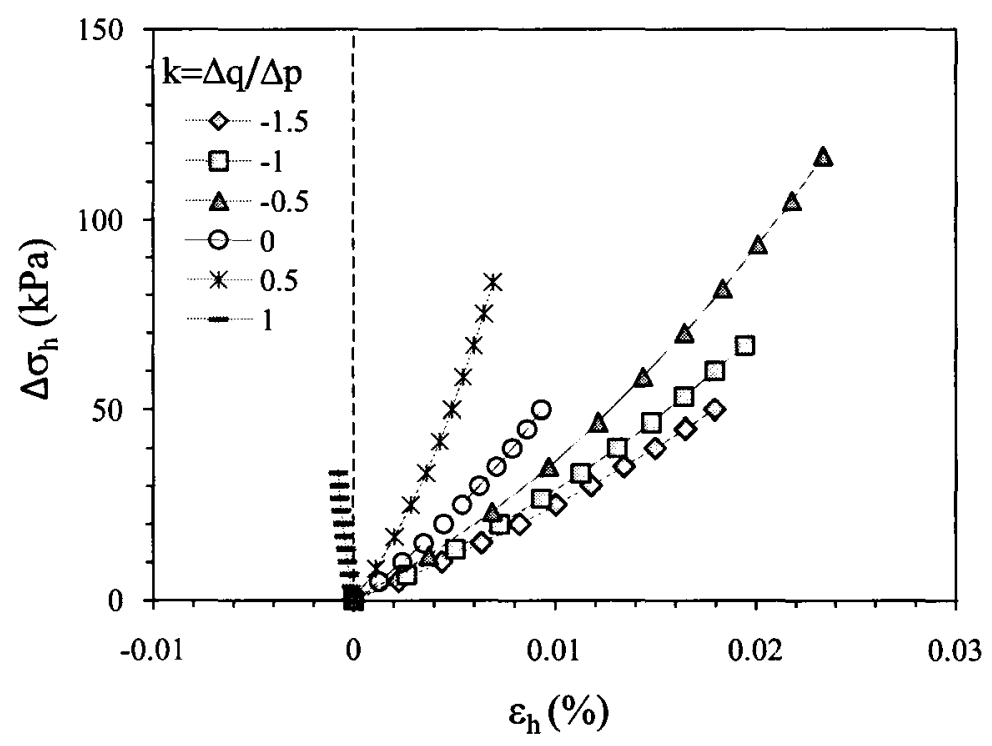

(a)

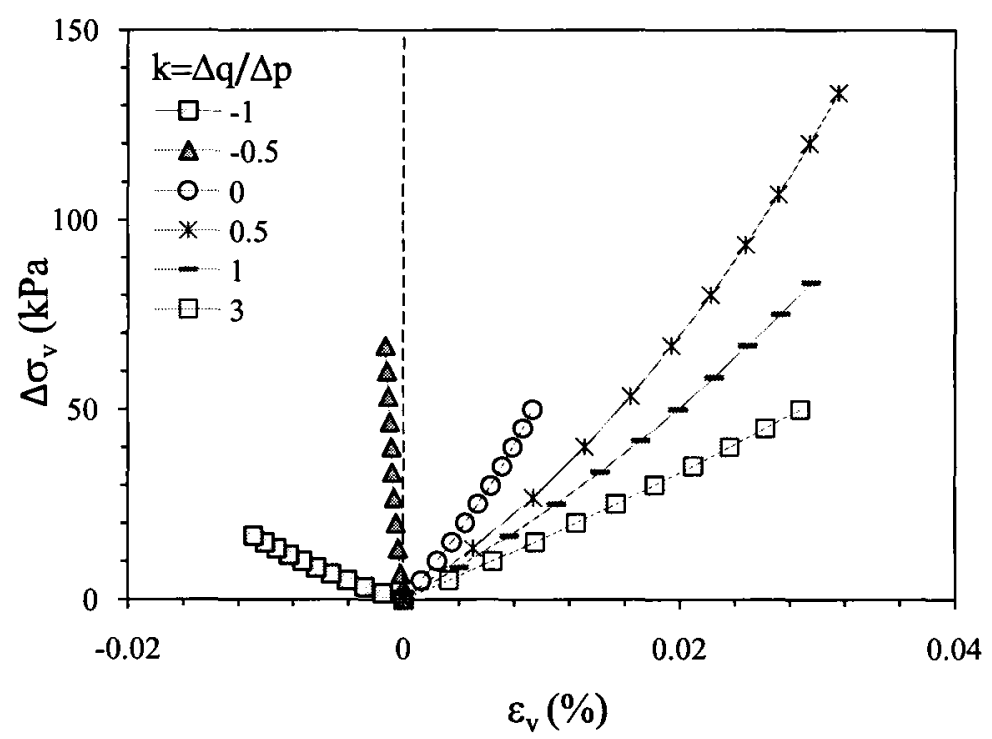

(b)

Fig. 4.2 Predicted responses of a nonlinear isotropic material (continued)

$$
\begin{gathered}
E=20 p^{0.515}(\mathrm{MPa}) \quad v=0.3 \\
\sigma_{d}=50 \mathrm{kPa} \quad \sigma_{c}=50 \mathrm{kPa}
\end{gathered}
$$




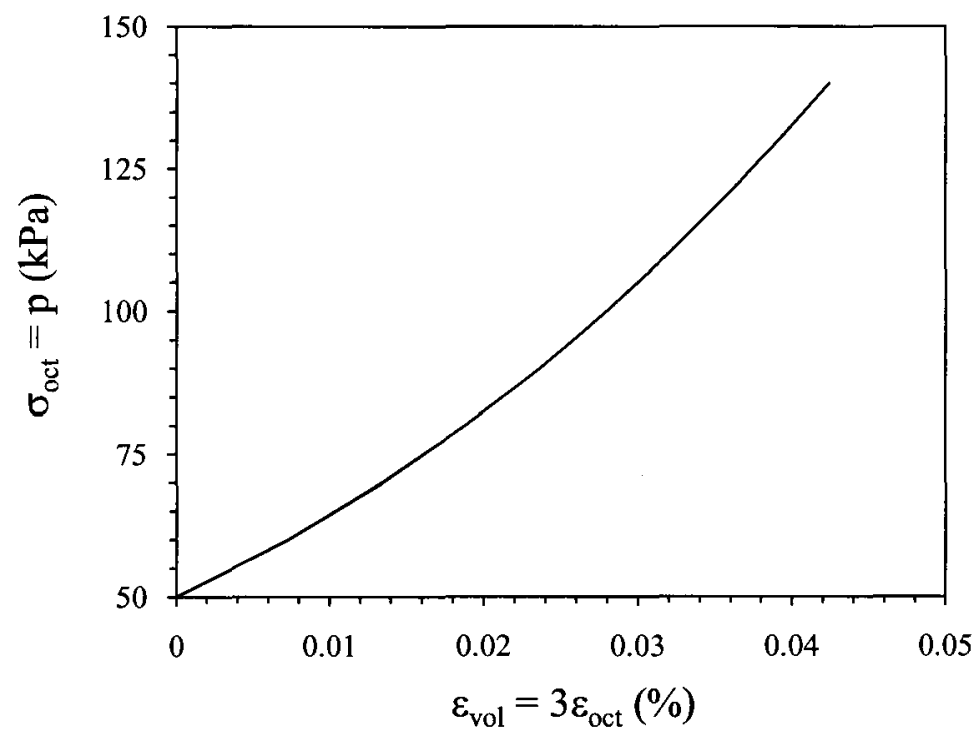

(c)

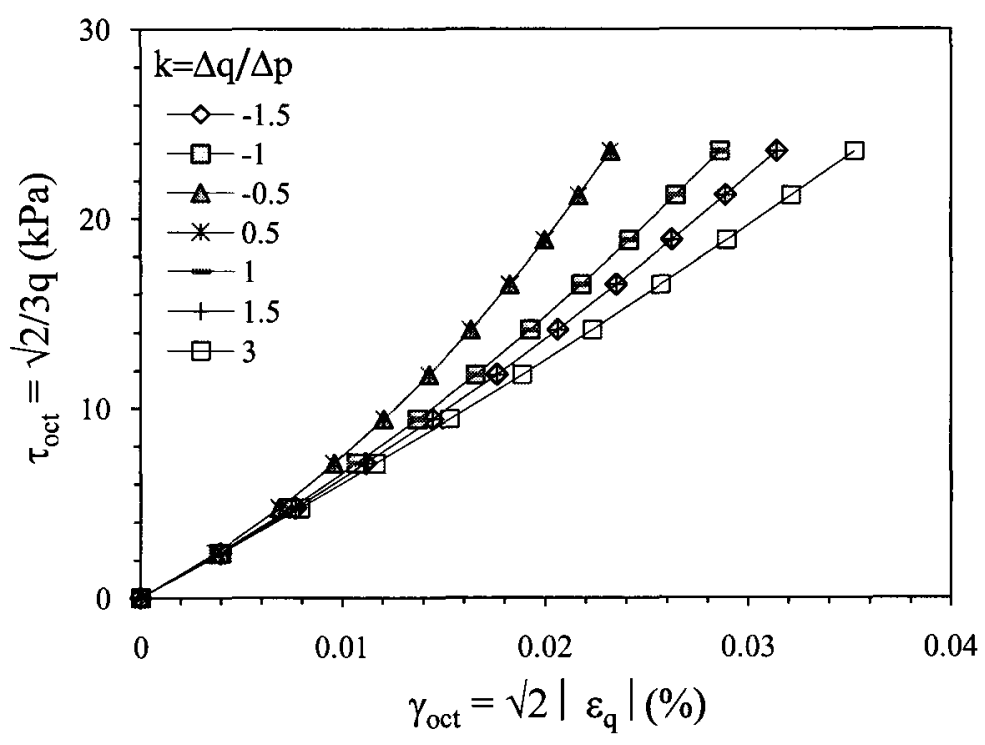

(d)

Fig. 4.2 Predicted responses of a nonlinear isotropic material

$$
\begin{gathered}
E=20 p^{0.515}(\mathrm{MPa}) \quad v=0.3 \\
\sigma_{d}=50 \mathrm{kPa} \quad \sigma_{c}=50 \mathrm{kPa}
\end{gathered}
$$


(b) Linear anisotropic material

The cross-anisotropic elastic constitutive relation is expressed as

$$
\left[\begin{array}{ccccc}
\frac{1}{E_{v}} & -\frac{v_{h v}}{E_{h}} & -\frac{v_{h v}}{E_{h}} & & \\
-\frac{v_{v h}}{E_{v}} & \frac{1}{E_{h}} & -\frac{v_{h h}}{E_{h}} & & \\
-\frac{v_{v h}}{E_{v}} & -\frac{v_{h h}}{E_{h}} & \frac{1}{E_{h}} & & \\
& & & \frac{1}{G_{v h}} & \\
& & & \frac{1}{G_{v h}} & \\
& & & \frac{1}{G_{h h}}
\end{array}\right]\left[\begin{array}{l}
\Delta \sigma_{z z} \\
\Delta \sigma_{x x} \\
\Delta \sigma_{y y} \\
\Delta \tau_{x z} \\
\Delta \tau_{y z} \\
\Delta \tau_{x y}
\end{array}\right]=\left[\begin{array}{l}
\Delta \varepsilon_{z z} \\
\Delta \varepsilon_{x x} \\
\Delta \varepsilon_{y y} \\
\Delta \gamma_{x z} \\
\Delta \gamma_{y z} \\
\Delta \gamma_{x y}
\end{array}\right]
$$

where the $x$ - $y$ plane corresponds to the 2 orthogonal directions in the horizontal plane in which the material is isotropic and $z$ is the vertical direction. The $\Delta$ has been introduced here in front of stresses to reinforce the fact that the properties are obtained through stress probe experiments that induce changes in strain relative to same initial states. The symmetry of the elastic stiffness matrix requires (Love, 1927)

$$
\frac{v_{h v}}{E_{h}}=\frac{v_{v h}}{E_{v}}
$$

The shear modulus in horizontal plane, $G_{h h}$, can be related to $E_{h}$ and $v_{h h}$ via

$$
G_{h h}=\frac{E_{h}}{2\left(1+v_{h h}\right)}
$$

When replacing the Cartesian coordinates $(x, y, z)$ with cylindrical coordinates $(r, \theta, z)$, the shear modulus in vertical plane $G_{v h}$ can be determined independently from a hollow cylinder test where $\Delta \tau_{x z}=\Delta \tau_{x y}=0$, via 


$$
G_{v h}=\frac{\Delta \tau_{z \theta}}{\Delta \gamma_{z \theta}}
$$

For an axial symmetric system, in which we only consider direct stresses, Eq. (4.13) becomes

$$
\left[\begin{array}{ccc}
\frac{1}{E_{v}} & -\frac{v_{v h}}{E_{v}} & -\frac{v_{v h}}{E_{v}} \\
-\frac{v_{v h}}{E_{v}} & \frac{1}{E_{h}} & -\frac{v_{h h}}{E_{h}} \\
-\frac{v_{v h}}{E_{v}} & -\frac{v_{h h}}{E_{h}} & \frac{1}{E_{h}}
\end{array}\right]\left[\begin{array}{l}
\Delta \sigma_{z} \\
\Delta \sigma_{r} \\
\Delta \sigma_{\theta}
\end{array}\right]=\left[\begin{array}{l}
\Delta \varepsilon_{z} \\
\Delta \varepsilon_{r} \\
\Delta \varepsilon_{\theta}
\end{array}\right]
$$

where the four independent parameters, $E_{h}, E_{v}, v_{v h}$ and $v_{h h}$, can be determined when the stress and strain increments are known. Eq. (4.17) can be rearranged as

$$
\left[\begin{array}{cccc}
\Delta \sigma_{z} & -\left(\Delta \sigma_{\theta}+\Delta \sigma_{r}\right) & 0 & 0 \\
0 & -\Delta \sigma_{z} & \Delta \sigma_{r} & -\Delta \sigma_{\theta} \\
0 & -\Delta \sigma_{z} & \Delta \sigma_{\theta} & -\Delta \sigma_{r}
\end{array}\right]\left[\begin{array}{c}
\frac{1}{E_{v}} \\
\frac{v_{v h}}{E_{v}} \\
\frac{1}{E_{h}} \\
\frac{v_{h h}}{E_{h}}
\end{array}\right]=\left[\begin{array}{c}
\Delta \varepsilon_{z} \\
\Delta \varepsilon_{r} \\
\Delta \varepsilon_{\theta}
\end{array}\right]
$$

For tests under triaxial stress conditions, the stress and strain increments satisfy $\Delta \sigma_{\theta}=\Delta \sigma_{r}$ and $\Delta \varepsilon_{\theta}=\Delta \varepsilon_{r}$. When substituting $\Delta \sigma_{z}, \Delta \sigma_{r}, \Delta \varepsilon_{z}$, and $\Delta \varepsilon_{r}$ with $\Delta \sigma_{v}, \Delta \sigma_{h}$, $\Delta \varepsilon_{v}$, and $\Delta \varepsilon_{h}$, respectively, Eq. (4.18) becomes

$$
\left[\begin{array}{ccc}
\Delta \sigma_{v} & -2 \Delta \sigma_{h} & 0 \\
0 & -\Delta \sigma_{v} & \Delta \sigma_{h}
\end{array}\right]\left[\begin{array}{c}
\frac{1}{E_{v}} \\
\frac{v_{v h}}{E_{v}} \\
\frac{1-v_{h h}}{E_{h}}
\end{array}\right]=\left[\begin{array}{l}
\Delta \varepsilon_{v} \\
\Delta \varepsilon_{h}
\end{array}\right]
$$


Assume that $E_{v}=150 \mathrm{MPa}, E_{h}=50 \mathrm{MPa}, v_{v h}=0.3$, and $v_{h h}=0.1$, the responses of an ideal linear cross-anisotropic material subjected to various stress paths are predicted using Eq. (4.19), as shown by the summary of results presented in Fig. 4.3. In contrast to the linear isotropic material, one observes:

- The trends of $\sigma_{h} v s \varepsilon_{h}$ and $\sigma_{v} v s \varepsilon_{v}$ for various stress paths, in particular the trends of $\sigma_{h} v s \varepsilon_{h}$, are different from those for the linear isotropic materials, see Figs. 4.1(a) and (b), 4.3(a) and (b). This may be attributed to the anisotropic nature of the material.

- The stress-strain relations in terms of invariants are very different and are stresspath dependent. Figures 4.3(c) and (d) show that both the bulk modulus and shear modulus are direction and stress-path dependent, whereas the bulk modulus and shear modulus of a linear isotropic material are constants.

- The trends of $\varepsilon_{v o l} v s \gamma_{o c t}$ and $\varepsilon_{v} v s \varepsilon_{h}$ are different from those of isotropic materials given $v$ and $\iota$ (or $k$ ), see Figs. 4.1(e) and (f), 4.3(e) and (f). For the IC loading path, the volume change is coupled to shear strain due to the anisotropic nature of the material, with $\varepsilon_{v}$ being smaller than $\varepsilon_{h}$. There is however no shear strain coupled with volume change for the linear isotropic material subjected to IC compression as $\varepsilon_{v}$ and $\varepsilon_{h}$ are the same.

- The major principal strain is not necessarily co-axial with the major principal stress, such as stress paths with $k=0.5$ and 1.0 , for which the major principal strain appears in the direction of the minor principal stress, as reflected by Fig. 4.3(f). 


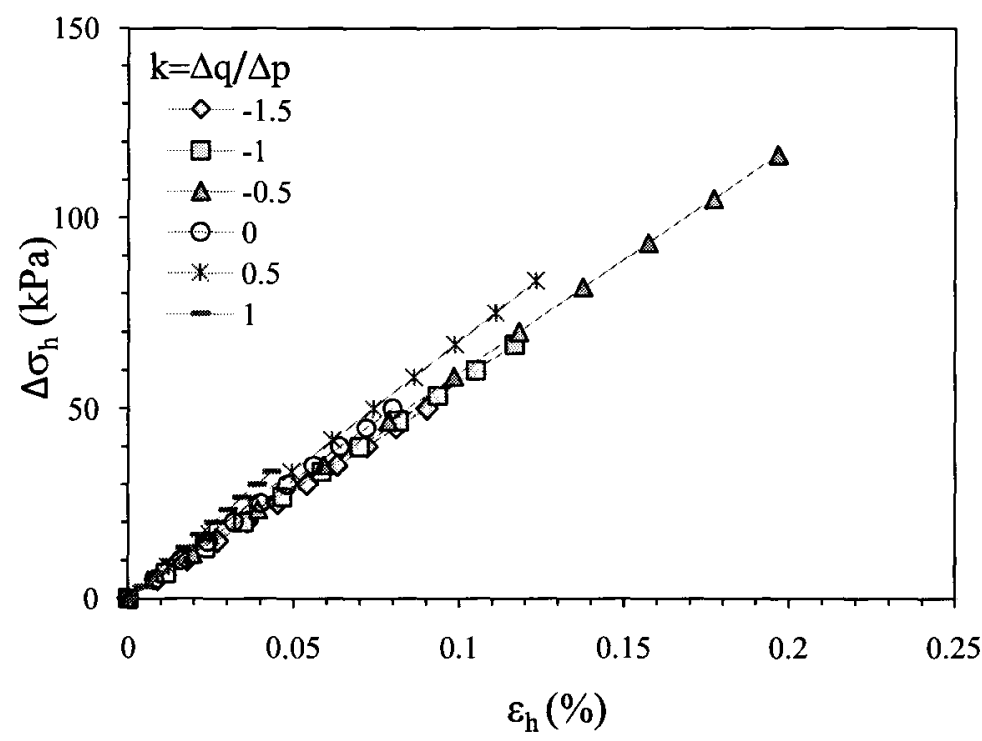

(a)

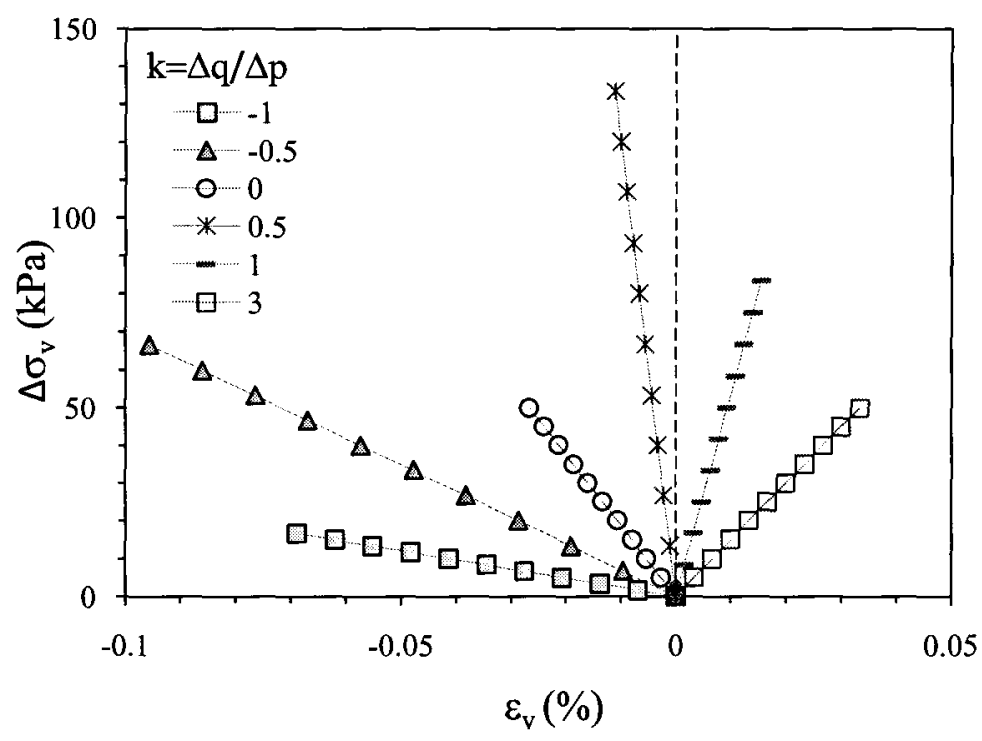

(b)

Fig. 4.3 Predicted responses of a linear anisotropic material (continued)

$$
\begin{gathered}
E_{v}=150 \mathrm{MPa} \quad E_{h}=50 \mathrm{MPa} \quad v_{v h}=0.3 \quad v_{h h}=0.1 \\
\sigma_{d}=50 \mathrm{kPa} \quad \sigma_{c}=50 \mathrm{kPa}
\end{gathered}
$$




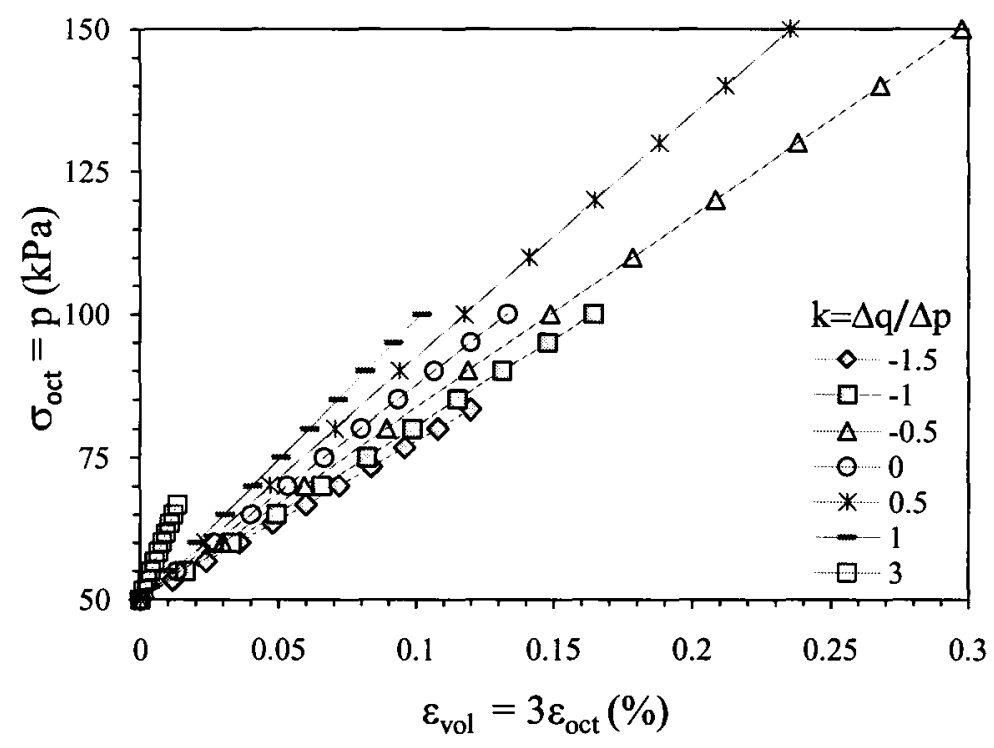

(c)

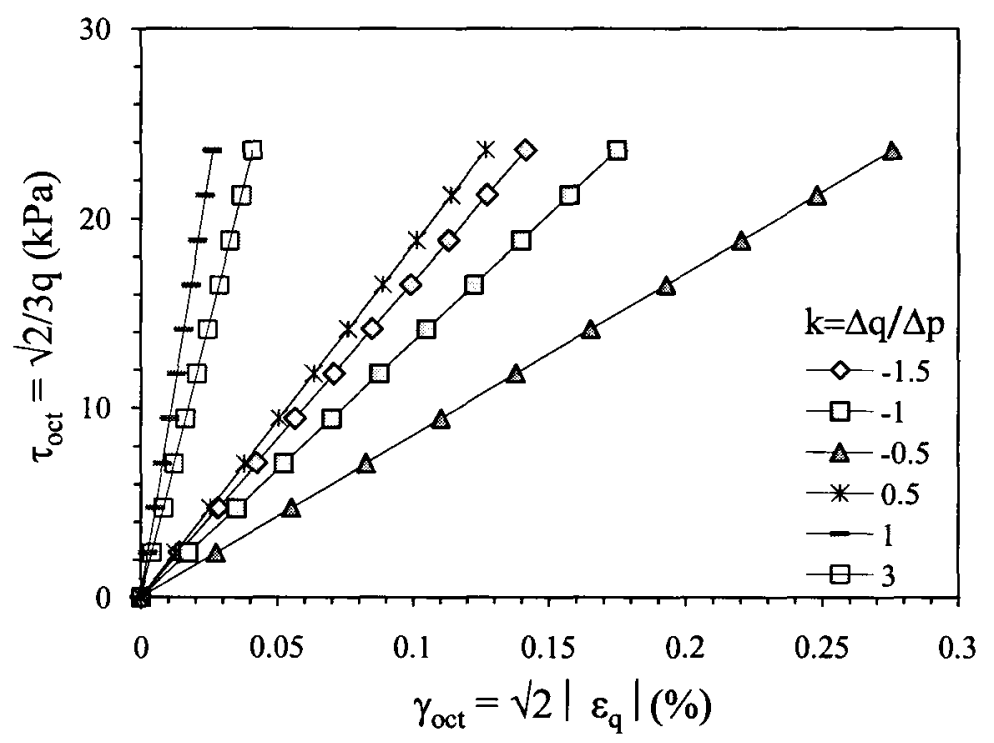

(d)

Fig. 4.3 Predicted responses of a linear anisotropic material (continued)

$$
\begin{gathered}
E_{v}=150 \mathrm{MPa} E_{h}=50 \mathrm{MPa} \quad v_{v h}=0.3 \quad v_{h h}=0.1 \\
\sigma_{d}=50 \mathrm{kPa} \quad \sigma_{c}=50 \mathrm{kPa}
\end{gathered}
$$




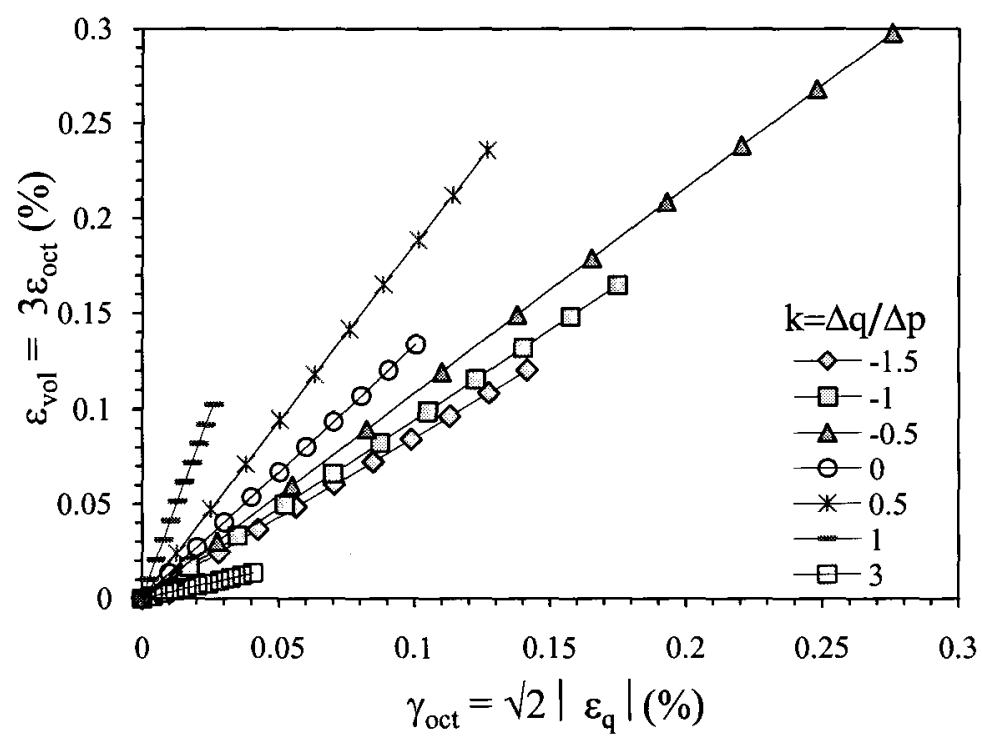

(e)

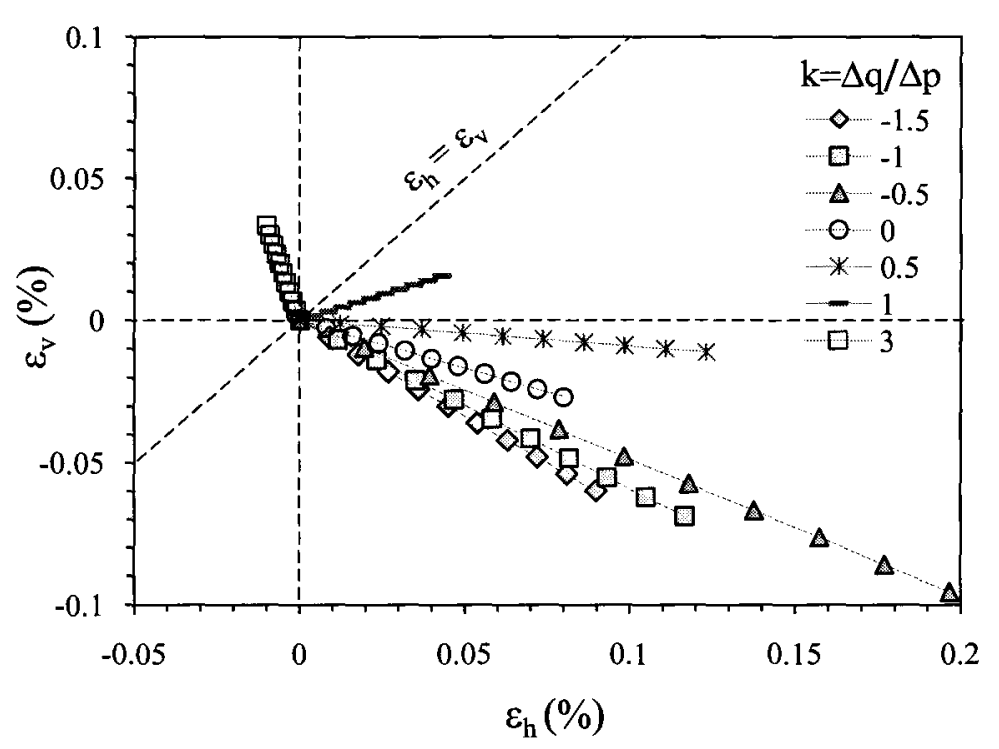

(f)

Fig. 4.3 Predicted responses of a linear anisotropic material

$$
\begin{gathered}
E_{v}=150 \mathrm{MPa} E_{h}=50 \mathrm{MPa} \quad v_{v h}=0.3 \quad v_{h h}=0.1 \\
\sigma_{d}=50 \mathrm{kPa} \quad \sigma_{c}=50 \mathrm{kPa}
\end{gathered}
$$




\section{(c) Test specimen}

Figure 4.4 presents typical responses of the first load cycle of a test specimen subjected to various stress paths under the same initial stress condition as idealized ones in previous sections. The effect of plasticity is small when compared to the resilient response. In comparison to the linear elastic isotropic/anisotropic and nonlinear elastic isotropic materials, one observes:

- The responses of the test specimen along various stress paths all show some degree of nonlinearity and display a resilient hysteresis loop during loading and unloading process. Hysteresis was also observed in later loading cycles. It should be noted for some cases where one observes large hysteresis loops, the resilient response however dominates. There is some similarity in the nonlinearity of the stress-strain relations with nonlinear isotropic materials. But all responses are stress-path dependent.

- An examination of the stresses and strains reveals that the relations of $\sigma_{v} v s \varepsilon_{v}$ and $\sigma_{h} v s \varepsilon_{h}$ depend on stress path. Therefore, the suggested use of tangent stiffness to characterize the resilient response of granular materials proposed by Konrad (2006) may not be accurate in reflecting the dependency of resilient modulus on stress path. Despite the nonlinearity, the trend of $\sigma_{h} v s \varepsilon_{h}$ is similar to those of the linear anisotropic material, with the dependency on stress path being less sensitive, as shown when comparing Figs. 4.1(a), 4.2(a), 4.3(a) and 4.4(a). This suggested that the test specimen's properties in the horizontal directions may be less influenced by stress path, which would need further investigation. 


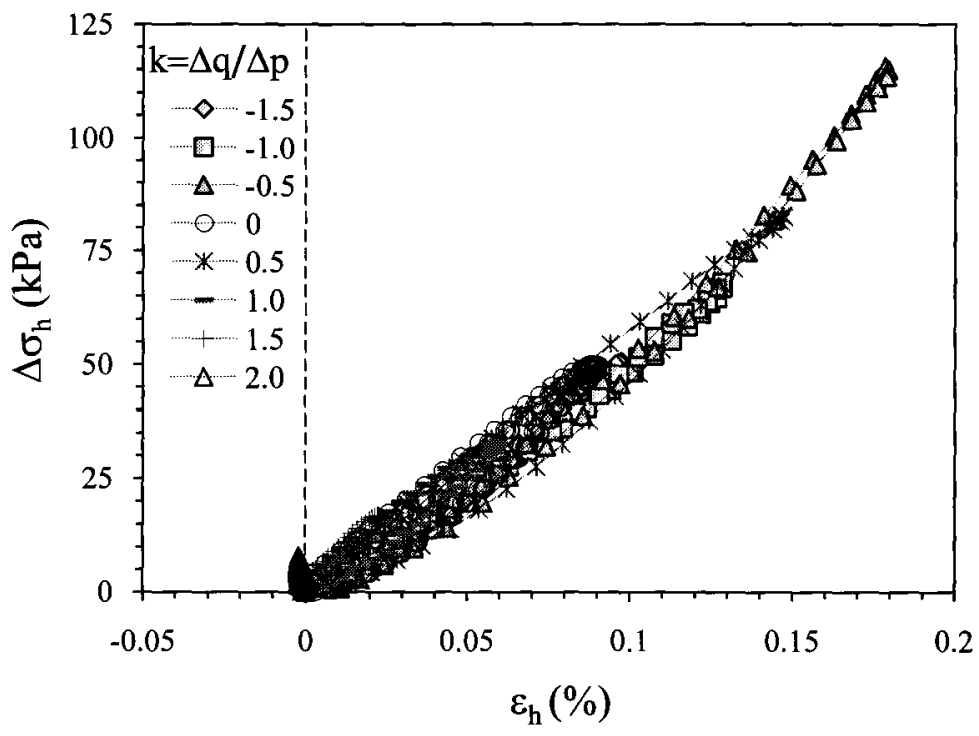

(a)

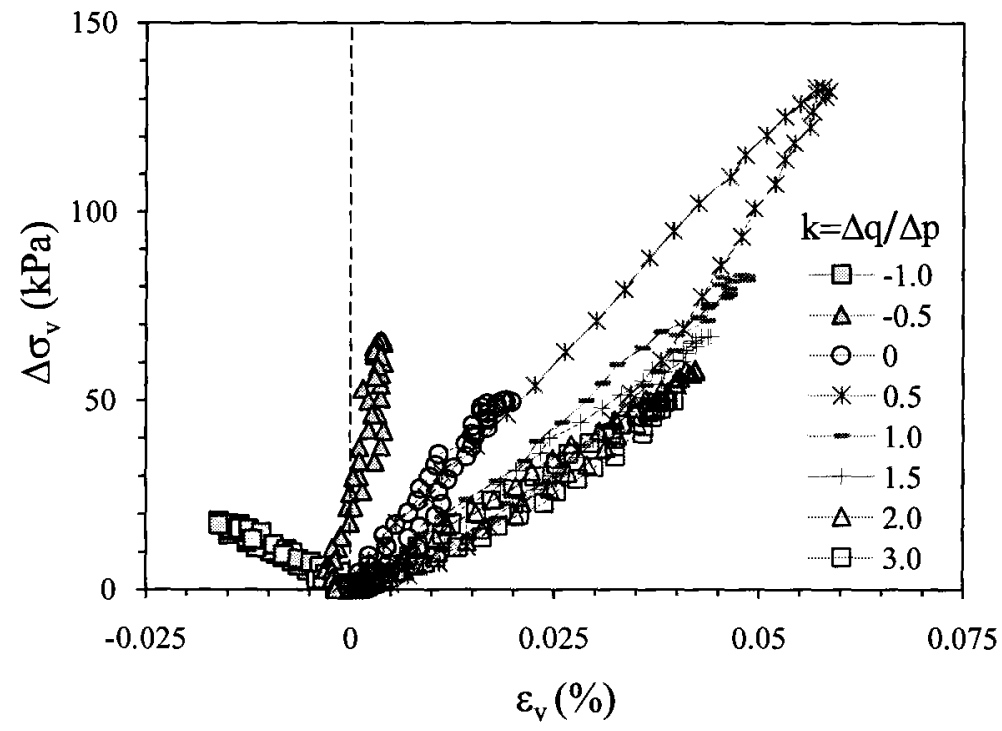

(b)

Fig. 4.4 The first cycle response loop of test specimen (continued)

$$
\sigma_{d}=50 \mathrm{kPa} \quad \sigma_{c}=50 \mathrm{kPa}
$$




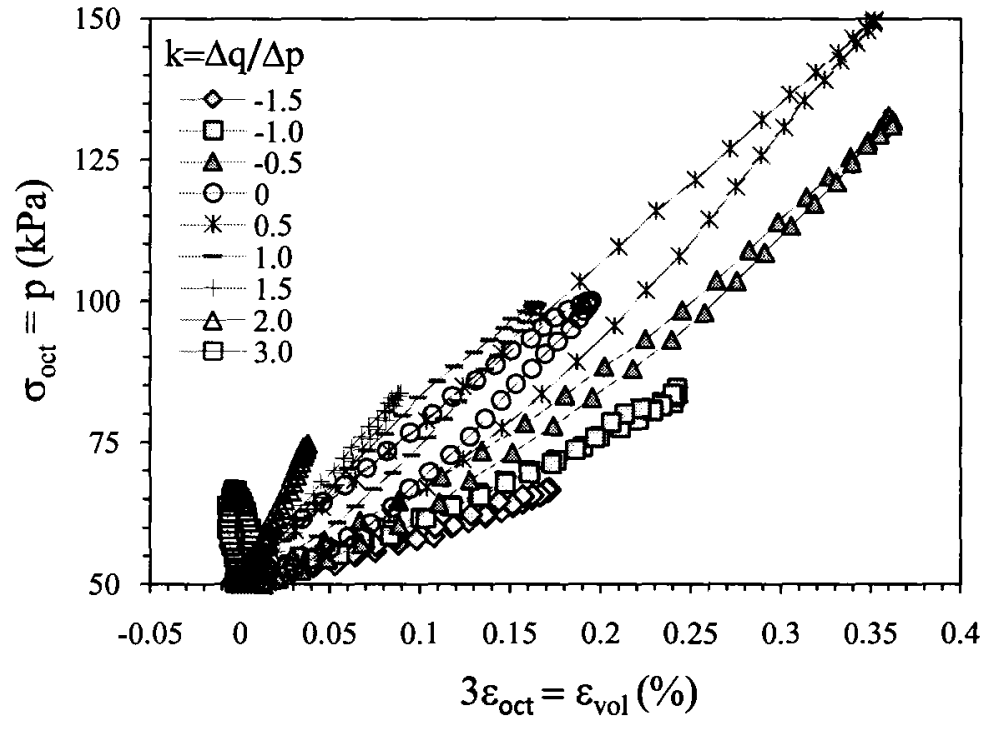

(c)

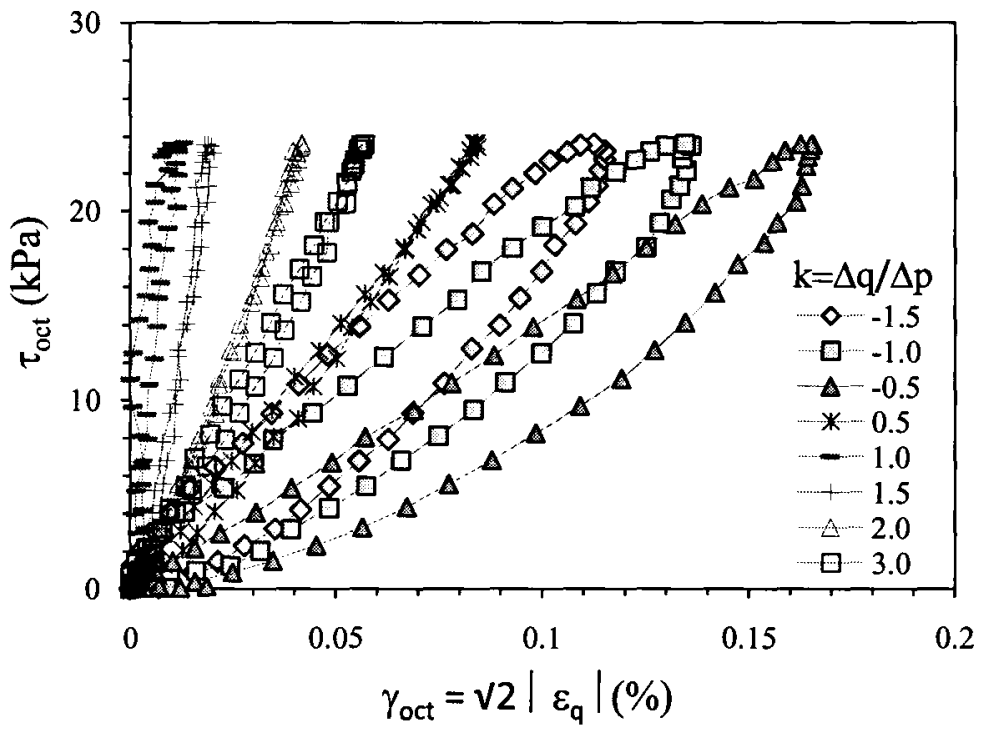

(d)

Fig. 4.4 The first cycle response loop of test specimen (continued)

$$
\sigma_{d}=50 \mathrm{kPa} \quad \sigma_{c}=50 \mathrm{kPa}
$$




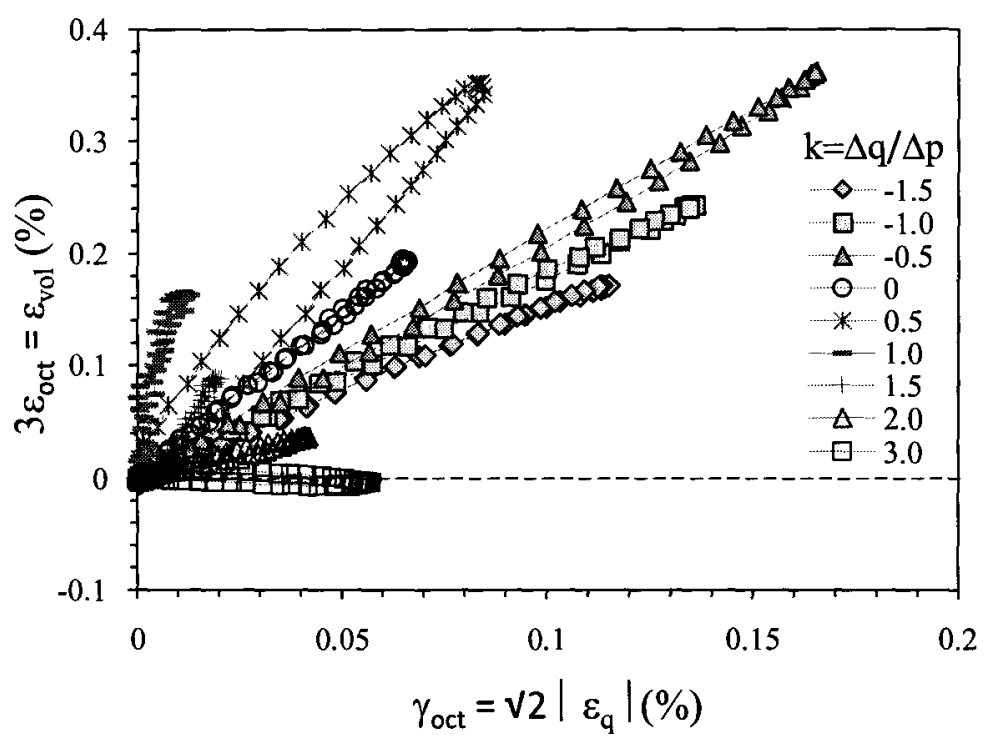

(e)

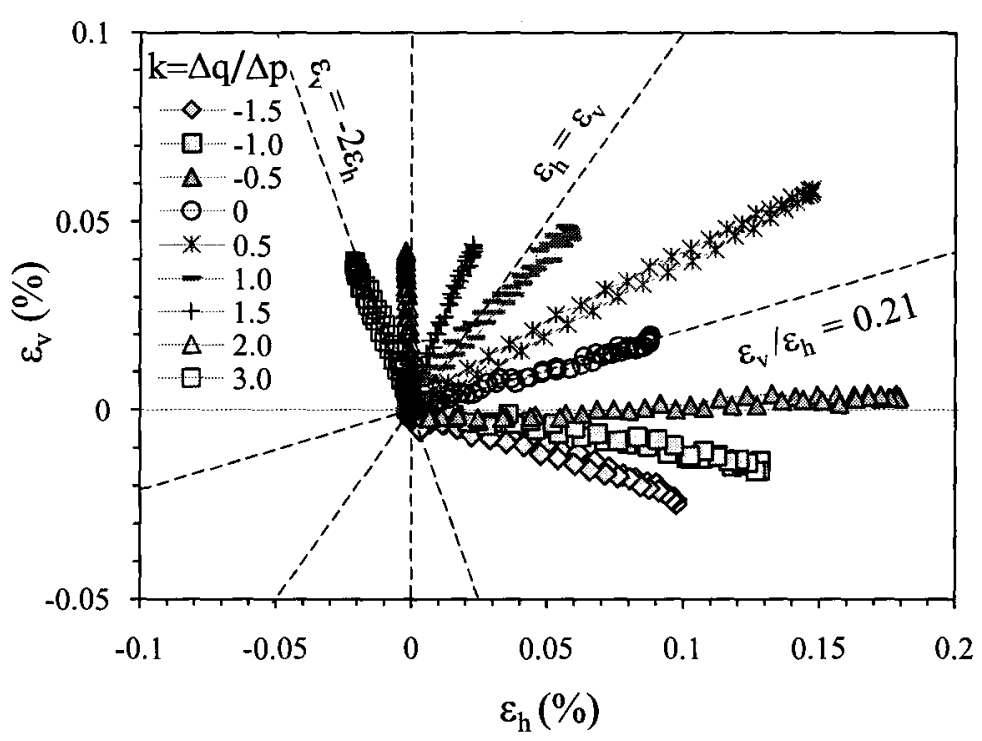

(f)

Fig. 4.4 The first cycle response loop of test specimen

$$
\sigma_{d}=50 \mathrm{kPa} \quad \sigma_{c}=50 \mathrm{kPa}
$$


- Figures 4.4(c) and (d) reveal that the different resilient bulk modulus and shear modulus are obtained when the stress path is changed due to the anisotropic nature of the material. In general, the bulk modulus for stress paths with $k \geq 0$ is higher than those having $k<0$. However, if resilient dilatancy occurs, negative bulk modulus is observed, such as for stress paths having $k=3.0$.

- The trends of $\varepsilon_{v o l} v s \gamma_{o c t}$ and $\varepsilon_{v} v s \varepsilon_{h}$ are stress-path dependent, which are similar to those of the linear anisotropic material, but the variations are nonlinear. In addition, resilient dilatancy may be induced by large deviator/shear stress for some stress paths with $k=2.0$ and 3.0 , which is however impossible for the idealized isotropic elastic material.

- For the IC loading path, volume change is coupled to shear strain due to material anisotropy, which is similar to that for linear anisotropic material.

- The major principal strain is not necessarily co-axial with the major principal stress, which is consistent to observations made for the linear anisotropic material.

Scrutinizing the experimental results and the predicted responses of the idealized materials, one concludes that the difference in the resilient responses of the specimen for various stress paths is attributed to the nonlinear anisotropic nature of the material.

For all stress paths applied within the framework of this research, there are noticeable differences in the patterns of deformation responses. As shown in Figs. 4.4(a), (b) and (f), for the IC loading paths with $\Delta q=0$, the material is consistently contractive in both vertical and horizontal directions despite the difference in the magnitude of 
horizontal and vertical strains. For $k>0$, the material is contractive vertically, but may be either contractive or expansive depending on the slope of stress paths and the material's anisotropy. When the ratio of $\Delta \sigma_{v} / \Delta \sigma_{h}$ is small (such as $k=0.5$ ), both $\sigma_{v}$ and $\sigma_{h}$ increase with $\sigma_{v}$ only increasing slightly faster than $\sigma_{h}$, while the lateral confinement is high enough to resist the vertical load, the specimen is contracive in both the vertical and horizontal directions. For a stress path with larger $k$, such as $k=2.0$ or 3.0 with higher $\Delta \sigma_{v} / \Delta \sigma_{h}$, the much quicker increase in $\sigma_{v}$ can induce lateral expansion of the specimen, which is similar to what is normally encountered in axial compression tests. For the case of larger deviator stress where plasticity develops, one can also have shearinduced volume change causing dilation.

Similarly, for negative $k$ stress paths that correspond to a more rapid increase in $\sigma_{h}$ than $\sigma_{v}$, the specimen always has compressive deformation in the horizontal direction, while the vertical strain can be either compressive or extensive depending on the value of $k$. As shown in Fig. 4.4(f), a steeper stress path with negative $k$ can cause substantial extension of the specimen in the vertical direction.

The anisotropy of material behaviour can be seen clearly from Figs. 4.4(e) and (f), which show $\varepsilon_{v o l} v s \gamma_{o c t}$ loops and $\varepsilon_{v} v s \varepsilon_{h}$ loops obtained from stress path tests with different $k$, respectively. For isotropic compression with $k=0$ and $\Delta q=0$, one observes that the shear strain is induced and $\frac{\Delta \varepsilon_{v}}{\Delta \varepsilon_{h}} \approx 0.21$ (corresponding to $\frac{\Delta \varepsilon_{v o l}}{\Delta \varepsilon_{v}} \approx 10.7$ ) is much smaller than $\frac{\Delta \varepsilon_{v}}{\Delta \varepsilon_{h}}=1$ (corresponding to $\frac{\Delta \varepsilon_{v o l}}{\Delta \varepsilon_{v}}=3$ for isotropic materials), which implies that the specimen is highly anisotropic and the deformation in the horizontal direction is 
much higher than that in the vertical direction. Unfortunately, the effect of anisotropy on material response cannot be easily visualized for other stress paths involving variations of both $\sigma_{v}$ and $\sigma_{h}$, due to the fact that the effect of material anisotropy and the effect of anisotropic stresses on the deformation response are coupled and cannot be distinguished and separated visually.

Similar trends of stress-strain responses are observed for each stress path at different confining pressures. However, an increase of initial cell pressure can increase the resistance to deformation as one would expect and reduce the material's anisotropy. Figure 4.5 shows the $\varepsilon_{v} v s \varepsilon_{h}$ loops obtained from tests along stress paths of different $k$ at an initial confining pressure of $150 \mathrm{kPa}$. For isotropic compression, it can be seen that $\frac{\Delta \varepsilon_{v}}{\Delta \varepsilon_{h}} \approx 0.24$ (corresponding to $\frac{\Delta \varepsilon_{v o l}}{\Delta \varepsilon_{v}} \approx 9.4$ ) is slightly larger than $\frac{\Delta \varepsilon_{v}}{\Delta \varepsilon_{h}} \approx 0.21$ (corresponding to $\frac{\Delta \varepsilon_{v o l}}{\Delta \varepsilon_{v}} \approx 10.7$ ) for $\sigma_{c}=50 \mathrm{kPa}$, which indicates that the difference between the horizontal strain and the vertical strain appears to decrease. This would suggest that the material becomes less anisotropic when the initial confining pressure increases. The conclusion is consistent with the results for other stress paths shown in Fig. 4.5. For example, when $k=1.0, \frac{\Delta \varepsilon_{v}}{\Delta \varepsilon_{h}}<1$ at $\sigma_{c}=50 \mathrm{kPa}$, while $\frac{\Delta \varepsilon_{v}}{\Delta \varepsilon_{h}}>1$ at $\sigma_{c}=150 \mathrm{kPa}$, implying that the material anisotropy is reduced as a result of increased cell pressure. 


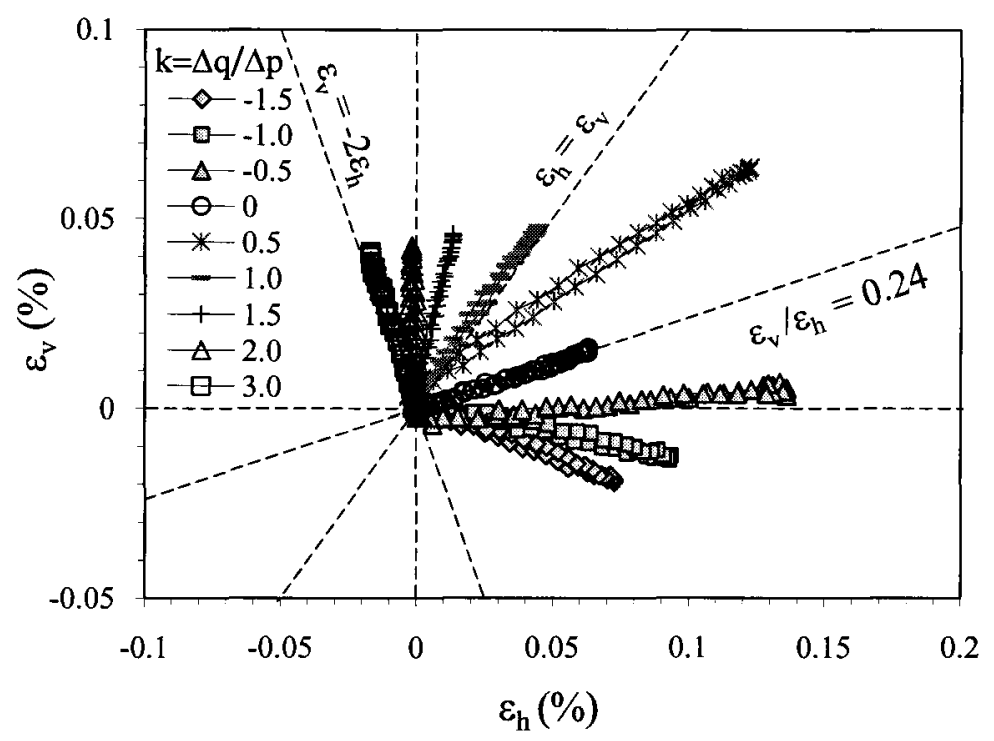

Fig. 4.5 Strain loops for various stress paths

$$
\sigma_{c}=150 \mathrm{kPa}, \sigma_{d}=75 \mathrm{kPa}
$$

\subsubsection{Resilient stresses and strains}

Figure 4.6 presents typical resilient responses for all stress paths at an initial cell pressure of $50 \mathrm{kPa}$. The variations of $\sigma_{d}\left(=\sigma_{1}-\sigma_{3}\right.$; i.e., $\left(\sigma_{v}-\sigma_{h}\right)$ for $k>0$ and $\left(\sigma_{h}-\sigma_{v}\right)$ for $k<0$ ) with the resilient strain $\varepsilon_{r}^{\text {major }}$ developed in the major principal stress direction (i.e., $\varepsilon_{v}$ for $k>0$ and $\varepsilon_{h}$ for $k<0$ ), are shown in Fig. 4.6(a). As expected, $\varepsilon_{r}^{\text {major }}$ increases as $\sigma_{d}$ increases. In general, the relation of $\sigma_{d} v s \varepsilon_{r}^{\text {major }}$ is affected by stress path. For example, for the same value of $\sigma_{d}, \varepsilon_{r}^{\text {major }}$ for $k<0$ is consistently larger than that for $k>0$ whereas according to Eqs. (4.1) and (4.2), $\varepsilon_{r}^{\text {major }}$ for $k<0$ is consistently smaller than that of $k>0$ for an idealized isotropic material. This trend clearly indicates that the test specimen is anisotropic and has a strong fabric in the vertical direction, as one might expect. 


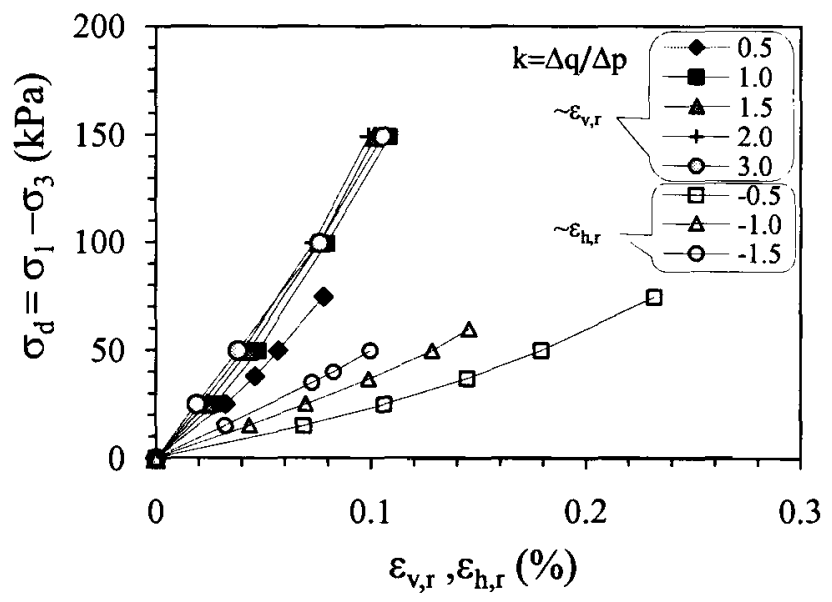

(a)

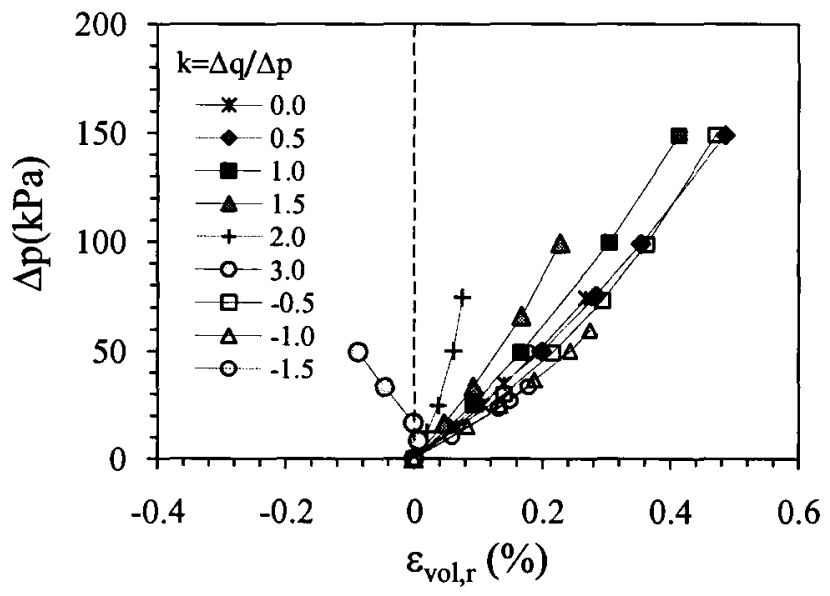

(b)

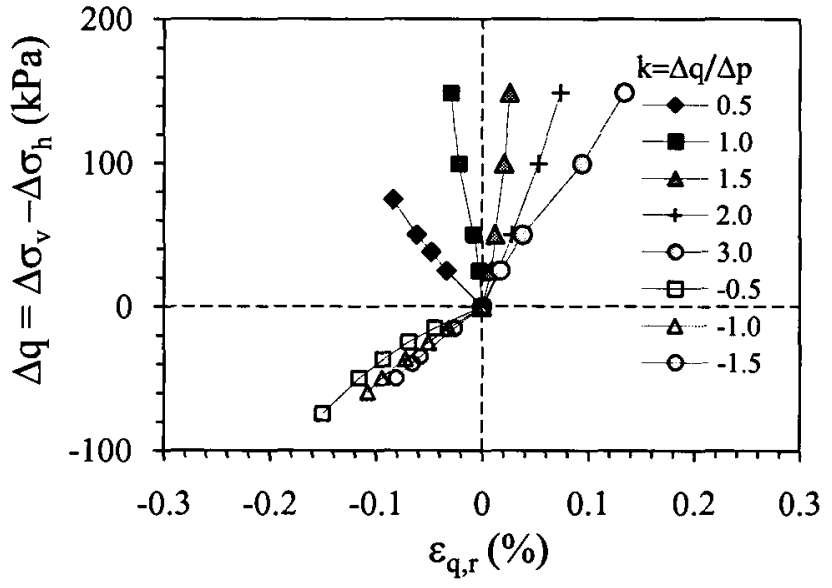

(c)

Fig. 4.6 Resilient stresses and resilient strains

$$
\sigma_{c}=50 \mathrm{kPa}
$$


The $\sigma_{d} v s \varepsilon_{v, r}$ relation for $k>0$ does not vary much when the stress path is changed. In comparison, the relation of $\sigma_{d} v s \varepsilon_{h, r}$ for $k<0$ varies with the stress path. The more significant change in the relation of $\sigma_{d} v s \varepsilon_{h, r}$ for $k<0$, on the other hand, implies that the influence of stress path on the resilient response is affected by the relative direction between the major principal stress and the direction of deposition. In other words, inherent fabric is a factor that should be examined when stress path effect is concerned.

Figure 4.6(b) presents the relations of $\Delta p v s \varepsilon_{v o l, r}$. One observes significant change in material response for different stress paths, which again indicates the influence of stress path and inherent fabric on material deformation properties. The relations of $\Delta q v s \varepsilon_{q, r}$ presented in Fig. 4.6(c) confirm this conclusion. In other words, the resilient bulk and shear moduli both have strong stress-path dependency. One observes that in Fig. 4.6(c) the dilatant resilient volumetric strain develops along the stress path with $k=3$ $\left(\frac{\Delta \sigma_{v}}{\Delta \sigma_{h}}=0\right)$, which is impossible for idealized isotropic elastic materials. This deformation phenomenon implies that fabric change takes place due to the increase in $\sigma_{v}$ while $\sigma_{h}$ stays constant. On the other hand, as shown in Fig. 4.6(c) $\varepsilon_{v, r}<\varepsilon_{h, r}$ is observed when $\Delta q=\Delta \sigma_{v}-\Delta \sigma_{h}>0$ along stress paths with $k=0.5\left(\frac{\Delta \sigma_{v}}{\Delta \sigma_{h}}=1.6\right)$ and $k=1.0\left(\frac{\Delta \sigma_{v}}{\Delta \sigma_{h}}=\right.$ 2.5), which implies that the maximum resilient strain does not necessarily develop in the direction of the major principal stress direction that is normal to the plane of deposition.

Figure 4.7 presents the resilient strain components for all stress path tests. In summary, there are 3 deformations patterns: 
- Contractive in both the vertical and horizontal directions, such as for $k=$ $-0.5 \sim 1.5$

- Contractive in the vertical direction, but dilative in the horizontal directions, such as for $k=2$ and 3 ; when the deviator stress exceeds a specific value, resilient dilatancy may be induced;

- Dilative in the vertical direction, but contractive in the horizontal directions, such as for $k=-1.0$ and -1.5 etc.

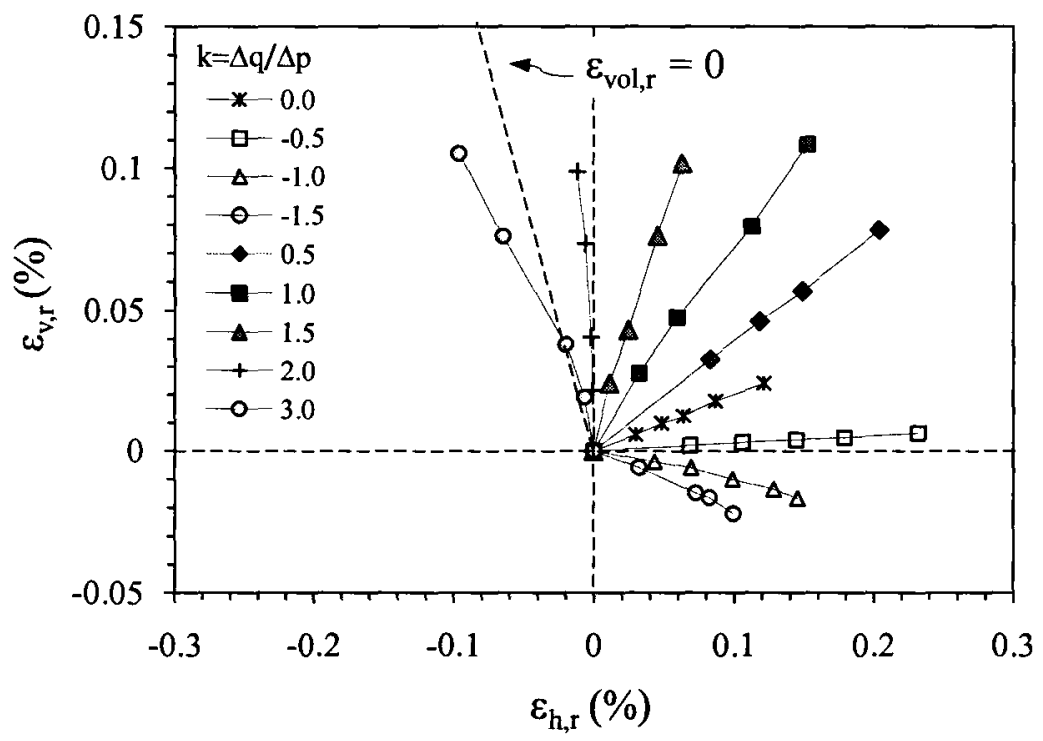

Fig. 4.7 Resilient strain components for all stress paths

$$
\sigma_{c}=50 \mathrm{kPa}
$$

Moreover, strain components for stress paths with $k=-0.5$ and 2 imply that there could be another two possible deformation patterns in some scenarios:

- When $k=2$, contractive in the vertical direction, very small strain $(\sim 0.001 \%)$ in the horizontal directions; 
- When $k=-0.5$, contractive in the horizontal directions, very small strain $(\sim$ $0.001 \%)$ in the vertical direction.

The variation of $\varepsilon_{v, r} v s \varepsilon_{h, r}$ along different stress paths is due to the combined influence of Poisson effect, inherent anisotropy and deviator stress that contributes to shear-induced volume change. The change of resilient volumetric strains from compaction to dilation is also attributed to the coupling of these factors. It is interesting to note from Fig. 4.7 that for the IC test with $k=0$, the ratio of resilient strain components $\frac{\varepsilon_{v, r}}{\varepsilon_{h, r}} \approx \frac{1}{5}$, indicating that the specimen has stronger fabric in vertical direction.

Figure 4.8 presents the relations $\frac{\varepsilon_{v, r}}{\varepsilon_{h, r}}$ vs $\frac{\sigma_{1}}{\sigma_{3}}$ for $k>0$ and $\frac{\varepsilon_{h, r}}{\varepsilon_{v, r}} v s \frac{\sigma_{1}}{\sigma_{3}}$ for $k \leq 0$. One observes that the value of $\varepsilon_{h, r} / \varepsilon_{v, r}$ for the IC loading test at a given $\sigma_{c}$ is almost constant $(\neq 1)$, implying that changes in the soil structure/fabric may be very small. For $k=$ $0.5 \sim 1.0$, the variations $\varepsilon_{h, r} / \varepsilon_{v, r}$ are also small, implying that changes in soil fabric might not be significant. For stress paths with $k>0, \varepsilon_{v, r} / \varepsilon_{h, r}$ tends to become negative with an increase of $k$, which indicates the material is compressed in the vertical direction and tends to expand in the horizontal directions. Similarly, for stress paths with $k<0$, $\varepsilon_{h, r} / \varepsilon_{v, r}$ tends to become negative as $k$ decreases, indicating that the material is contractive in the horizontal directions and tends to expand in the vertical direction. The variation of $\varepsilon_{v, r} / \varepsilon_{h, r}$ or $\varepsilon_{h, r} / \varepsilon_{v, r}$ with $\sigma_{1} / \sigma_{3}$ for different stress paths implies that the fabric of the material undergoes certain changes when the material is sheared along various stress paths, in particular those where extensive strains, either in the vertical direction or in the horizontal directions, are observed. 


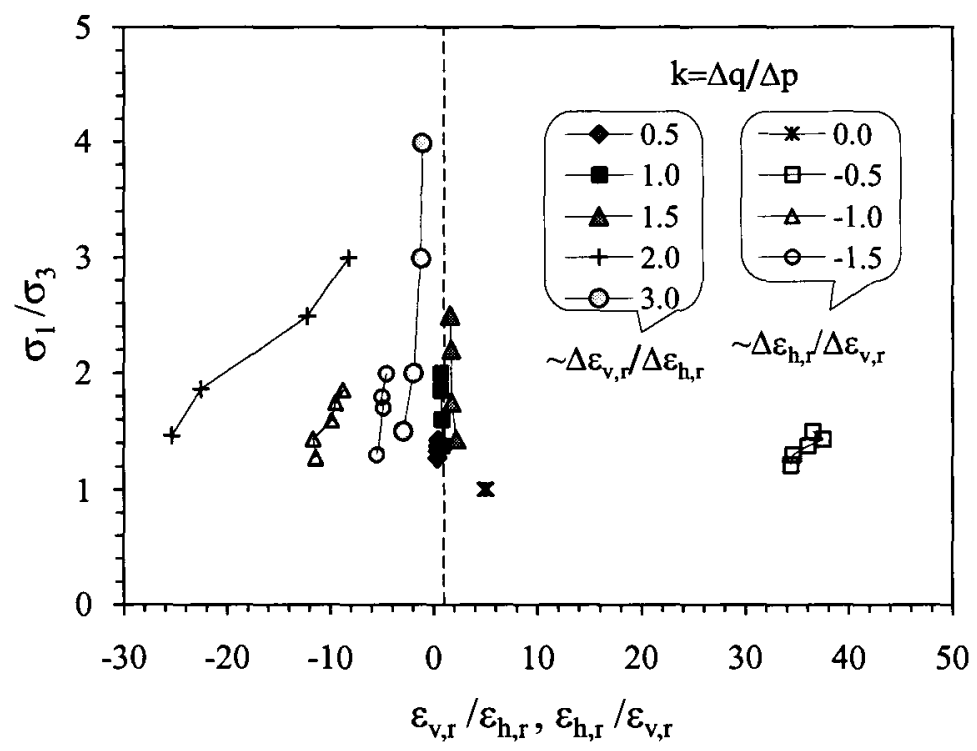

Fig. 4.8 Stress ratios applied and strain ratios for different stress paths $\sigma_{c}=50 \mathrm{kPa}$

\subsubsection{Effect of initial confining pressures}

For conventional resilient modulus tests $(k=3.0)$, the influence of cell pressure on the resilient modulus has long been recognized. As shown in Fig. 4.9, at a given repeated deviator stress, an increase in cell pressure tends to reduce the vertical resilient strains, which in turn leads to an increase in the resilient modulus. And the increased confinement tends to restrain lateral expansion of the specimen. As a result, the resilient dilative volumetric strain decreases when the initial confining pressure increases. Once again, the relations of $\Delta p v s \varepsilon_{v o l, r}$ suggest that the test material is anisotropic. When resilient dilatancy occurs, the resilient bulk modulus becomes negative and the Poisson's ratio $v_{v h}$ is greater than 0.5 . The variations of $\Delta q v s \varepsilon_{q, r}$ clearly show that the resilient shear modulus increases as confining pressure increases. 

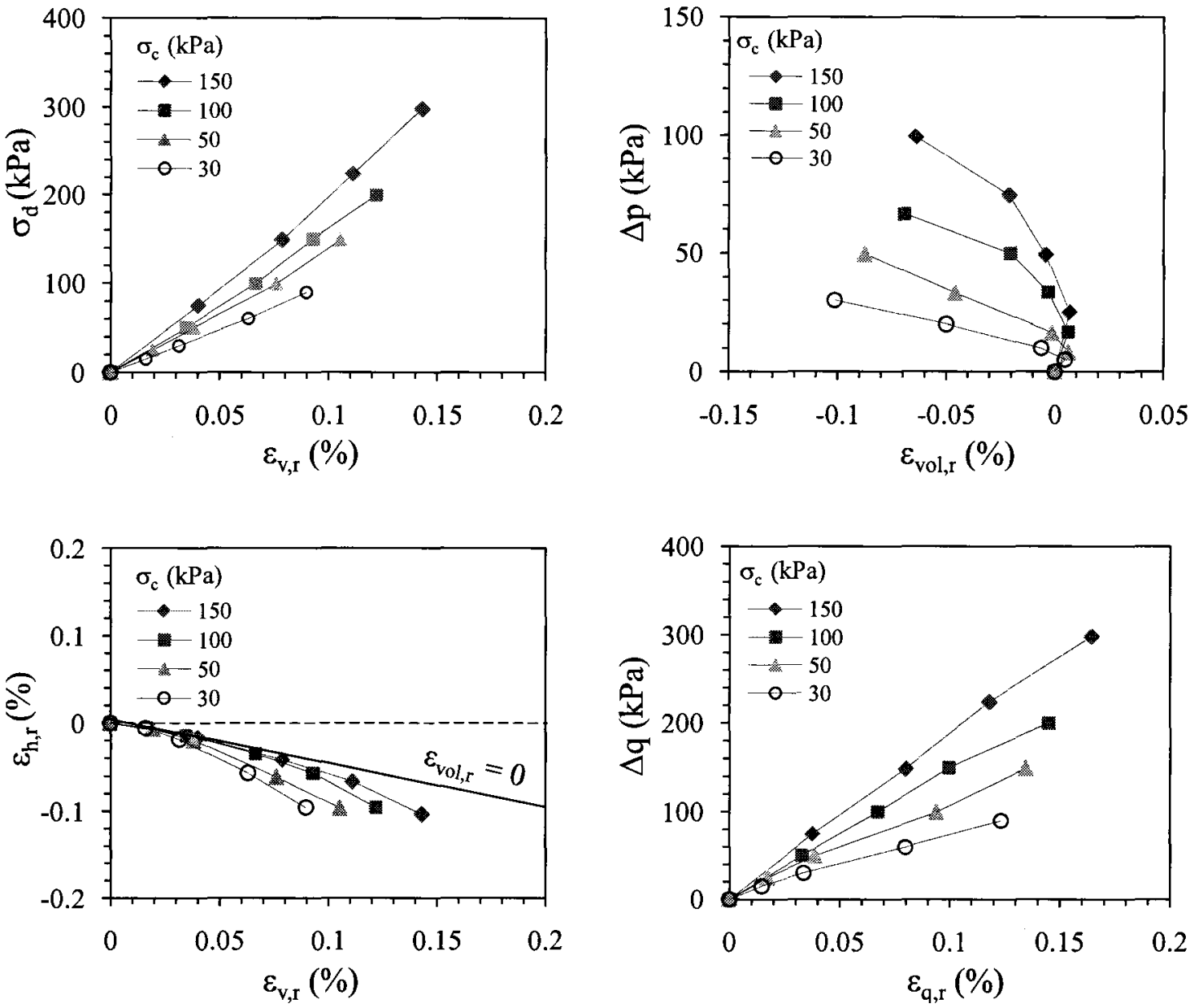

Fig. 4.9 Resilient stresses and resilient strains for $k=3.0$

For the other stress path tests $(k \neq 0)$, an increase in the initial cell pressure also improves the material's resistance to deformation. For example, Fig. 4.10 shows the variation of resilient strain with stress for the stress path of $k=-1.5$. Given constant $\sigma_{d}$, the resilient strains $\varepsilon_{h, r}$ and $\varepsilon_{v, r}$ become smaller when the material is sheared at a higher confining pressure, and the data clearly show increases in both bulk modulus and shear modulus as the confining pressure increases. 

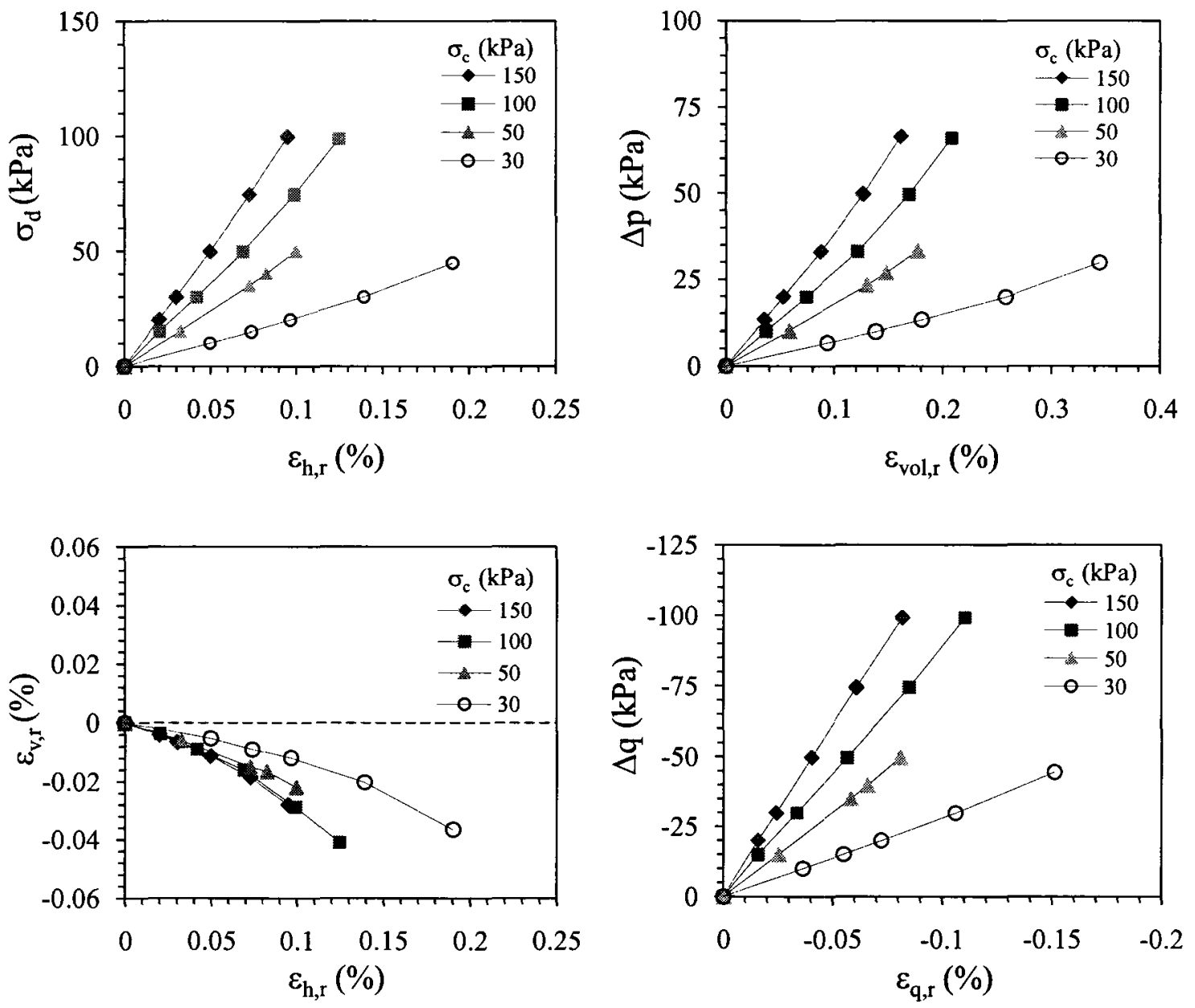

Fig. 4.10 Resilient stresses and resilient strains for $k=-1.5$

For the IC loading path, as seen in Fig. 4.11, for a given $\Delta \sigma_{\mathrm{c}}$, the measured resilient strains $\varepsilon_{h, r}$ and $\varepsilon_{v, r}$ decrease as $\sigma_{c}$ increases. Therefore the resilient bulk modulus increases as one expects. Moreover, the ratio of $\varepsilon_{h, r} / \varepsilon_{v, r}$ is consistently higher than unity, but decreases when the initial confinement increases, indicating that the inherent anisotropy of the material is reduced and the material becomes less anisotropic. This finding is consistent to the results obtained by AnhDan et. al. (2006), among others. 

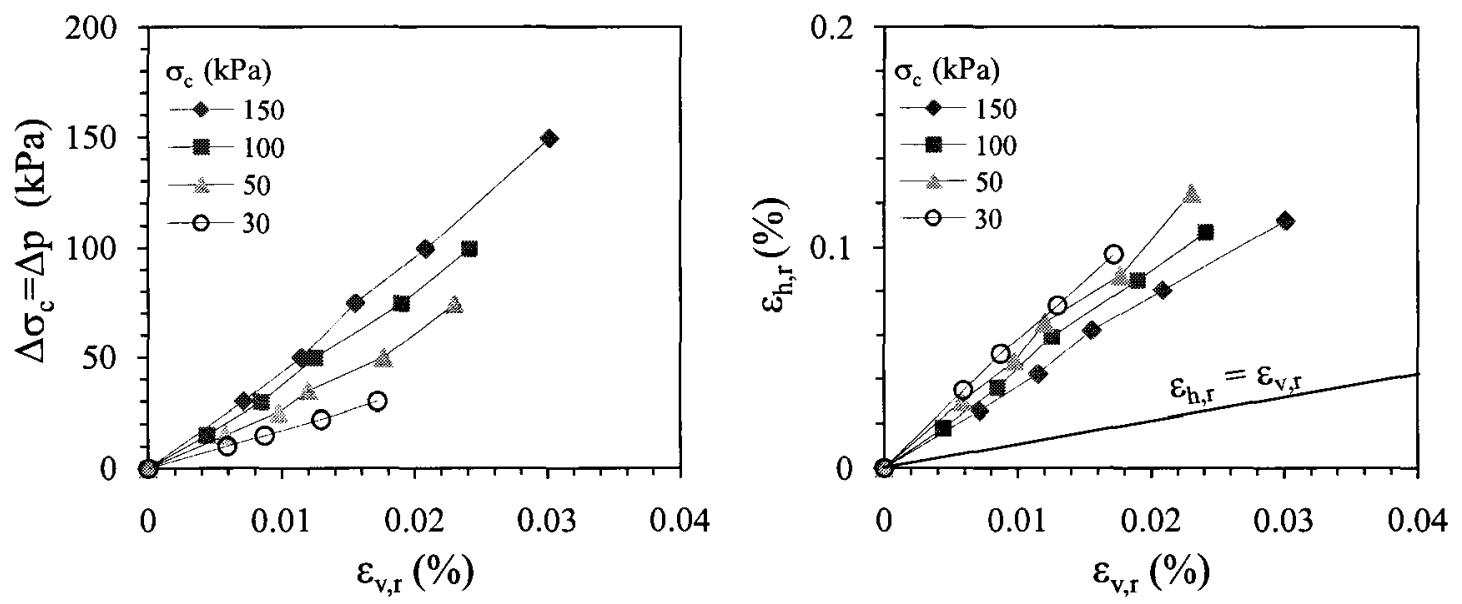

Fig. 4.11 Resilient stresses and resilient strains for IC loading

\subsection{DETERMINATION OF CROSS-ANISOTROPIC PROPERTIES}

The elastic properties can be determined from the measurement of stress increment and strain increment in triaxial tests. For CCP triaxial tests with $\Delta \sigma_{h}=0, v_{v h}$ and $E_{v}$ can be solved from Eq. (4.19) as

$$
\left[\begin{array}{ccc}
\Delta \sigma_{v} & 0 & 0 \\
0 & -\Delta \sigma_{v} & 0
\end{array}\right]\left[\begin{array}{c}
\frac{1}{E_{v}} \\
\frac{v_{v h}}{E_{v}}
\end{array}\right]=\left[\begin{array}{l}
\Delta \varepsilon_{v} \\
\Delta \varepsilon_{h}
\end{array}\right]
$$

Alternatively, one can plot the relations between $\Delta \sigma_{v} v s \Delta \varepsilon_{v}$ and $\Delta \varepsilon_{h} v s \Delta \varepsilon_{v}$ to obtain $E_{v}$ and $v_{v h}$, as shown in Fig. 4.12. For triaxial tests with $\Delta \sigma_{v}=0$, as shown in Fig. 4.13, one can only obtain the following relations from Eq. (4.19)

$$
\begin{gathered}
\frac{\Delta \sigma_{h}}{\Delta \varepsilon_{h}}=\frac{E_{h}}{1-v_{h h}} \\
\frac{\Delta \varepsilon_{v}}{\Delta \varepsilon_{h}}=\frac{\left(1-v_{h h}\right) E_{v}}{2 v_{v h} E_{h}}
\end{gathered}
$$


from which the values of $E_{h}$ and $v_{h h}$ are coupled and cannot be uniquely determined.

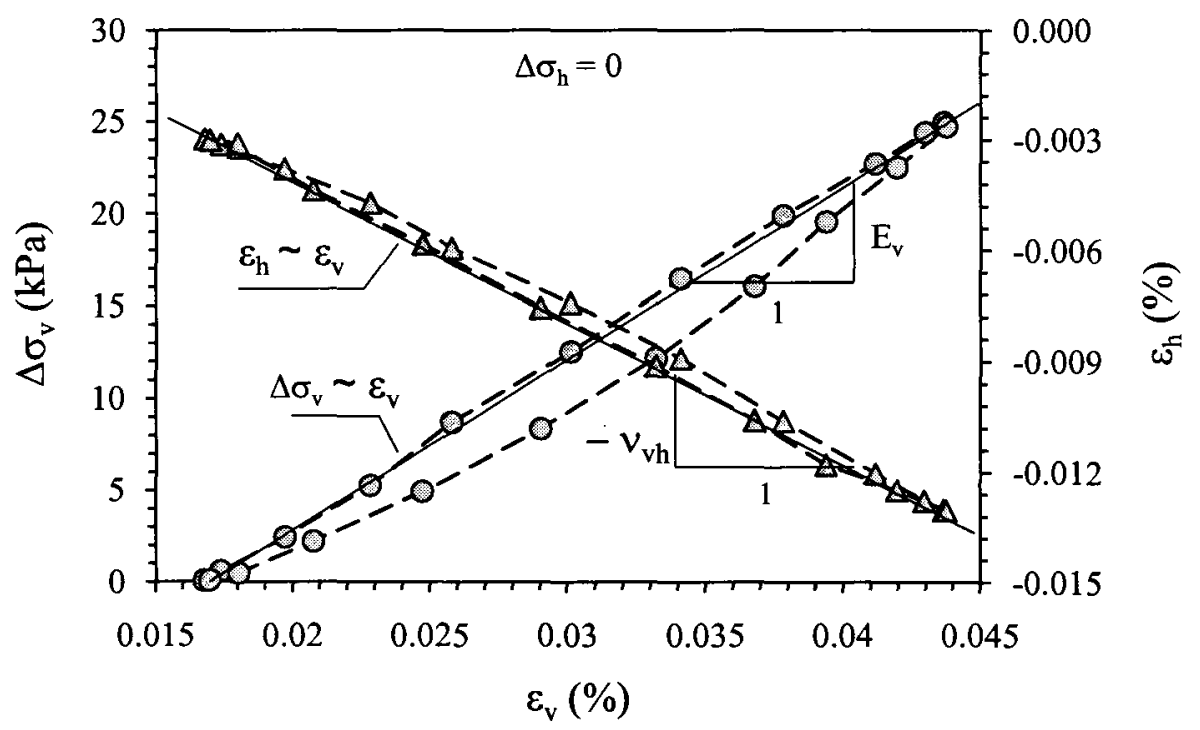

Fig. 4.12 Stress strain relationships for CCP test

$$
\sigma_{c}=50 \mathrm{kPa}
$$

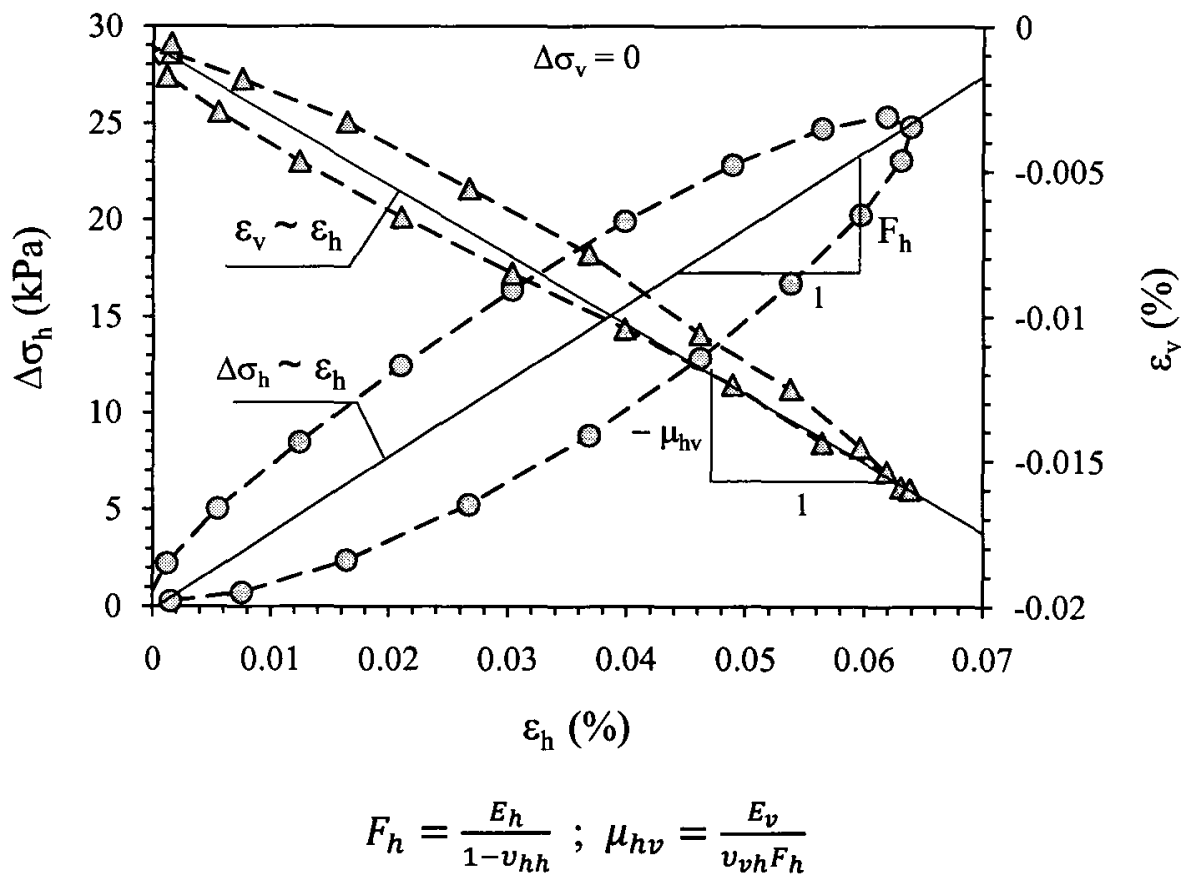

Fig. 4.13 Stress strain relationships for triaxial test $\sigma_{h}^{i}=50 \mathrm{kPa}$ (Initial horizontal stress) 
For trixial tests with $\Delta \sigma_{h} \neq 0$ and $\Delta \sigma_{v} \neq 0$, Eq. (4.19) is an ill-posed system since it has only two equations for four unknowns. Therefore, additional equations are needed to determine all of the cross-anisotropic elastic properties, which also requires necessary assumptions to decouple $v_{h h}$ and $E_{h}$ from $\frac{E_{h}}{1-v_{h h}}$.

Tuturmluer and Seyhan (2003) indicated that one can perform 3 slightly different but very close stress path tests (i.e., $k \pm 0.1$ and $k$, respectively) to obtain the required numbers of equations. Therefore, for each stress path test listed in Tables AI.1 and AI.2, 2 additional stress path tests were carried out. When applying Eq. (4.19) to each individual test, six equations can be obtained as follows

$$
\left[\begin{array}{ccc}
\Delta \sigma_{v}{ }^{1} & -2 \Delta \sigma_{h}{ }^{1} & 0 \\
0 & -\Delta \sigma_{v}{ }^{1} & \Delta \sigma_{h}{ }^{1} \\
\Delta \sigma_{v}{ }^{2} & -2 \Delta \sigma_{h}{ }^{2} & 0 \\
0 & \Delta \sigma_{v}{ }^{2} & \Delta \sigma_{h}{ }^{2} \\
\Delta \sigma_{v}{ }^{3} & -2 \Delta \sigma_{h}{ }^{3} & 0 \\
0 & \Delta \sigma_{v}{ }^{3} & \Delta \sigma_{h}{ }^{3}
\end{array}\right]\left[\begin{array}{c}
\frac{1}{E_{v}} \\
\frac{v_{v h}}{E_{v}} \\
\frac{1-v_{h h}}{E_{h}}
\end{array}\right]=\left[\begin{array}{c}
\Delta \varepsilon_{v}{ }^{1} \\
\Delta \varepsilon_{h}{ }^{1} \\
\Delta \varepsilon_{v}{ }^{2} \\
\Delta \varepsilon_{h}{ }^{2} \\
\Delta \varepsilon_{v}{ }^{3} \\
\Delta \varepsilon_{h}{ }^{3}
\end{array}\right]
$$

Equation (4.23) can be written as

$$
A X=\varepsilon
$$

where, $\boldsymbol{A}, \boldsymbol{X}$ and $\boldsymbol{\varepsilon}$ can be identified when comparing Eqs. (4.23) and (4.24). Equation (4.24) is an over-determined system of linear equations. The least square method can be used to solve for the unknowns in $\boldsymbol{X}$ by solving $\boldsymbol{A}^{T} \varepsilon=\boldsymbol{A}^{T} \boldsymbol{A X}$.

After the three components, which include $\frac{1-v_{h h}}{E_{h}}$, in $X$ are determined, the following assumption (Graham and Houlsby 1983) is adopted to decouple $v_{h h}$ and $E_{h}$ 
from $\frac{1-v_{h h}}{E_{h}}$.

$$
\sqrt{\frac{E_{h}}{E_{v}}}=\frac{v_{h h}}{v_{v h}}
$$

The values of $E_{h}, E_{v}, v_{v h}$ and $v_{h h}$ that are determined from the experimental data must be further examined to ensure that the compliance matrix in Eq. (4.17) is positive definite, which is a fundamental requirement for non-negative strain energy. To fulfill this requirement, Pickering (1970) and Raymond (1970) have shown that elastic properties must satisfy the following inequalities

$$
\begin{gathered}
-1<v_{h h}<1 \\
\frac{E_{v}}{E_{h}}\left(1-v_{h h}\right)-2 v_{v h}^{2}>0
\end{gathered}
$$

In summary, the cross-anisotropic resilient properties of granular material for a specific stress path with constant $k$ are determined experimentally in this study as follows:

- Carry out 3 slightly different but very close stress path tests (i.e., $k \pm 0.1$ and $k$, respectively) to determine $E_{h}, E_{v}, v_{v h}$ and $v_{h h}$ according to Eqs. (4.24) and (4.25);

- Check the constraints of inequalities (4.26) and (4.27).

Table 4.1 presents sample calculations for cross-anisotropic properties from 2 stress path tests. A comparison between the measured and predicted deformations indicates that the approach employed in solving for resilient properties is satisfactory. The results 
obtained from the tests to check for the repeatability are also listed in Table 4.2. One observes that the resilient properties of "identical" samples are very close to each other for the same stress path.

Table 4.1 Samples calculation of cross-anisotropic properties

\begin{tabular}{|c|c|c|c|c|c|c|c|c|c|c|}
\hline Test code & $\sigma_{c}(k P a)$ & $\left(\Delta \sigma_{h}, \Delta \sigma_{v}\right)(k P a)$ & $\varepsilon_{h, r}(\%)$ & $\varepsilon_{v, r}(\%)$ & $\begin{array}{l}\text { Predicted } \\
\varepsilon_{v, r}(\%)\end{array}$ & $\begin{array}{l}\text { Predicted } \\
\varepsilon_{h, r}(\%)\end{array}$ & $M_{r}^{h}(M P a)$ & $M_{r}^{v}(M P a)$ & $v_{h h}$ & $v_{v h}$ \\
\hline A_CTC & 50 & $(0.15,50.07)$ & -0.02435 & 0.03692 & -0.02435 & 0.03692 & - & 135.6 & - & 0.66 \\
\hline \multirow{3}{*}{ A_CTC } & \multirow{3}{*}{50} & $(0.15,50.07)$ & 0.02435 & 0.03692 & -0.02439 & 0.03763 & 20.7 & 132.6 & 0.26 & 0.66 \\
\hline & & $(2.83,52.77)$ & -0.01629 & 0.03817 & -0.01617 & 0.03699 & & & & \\
\hline & & $(-2.75,47.21)$ & -0.03340 & 0.03778 & -0.03328 & 0.03834 & & & & \\
\hline \multirow{3}{*}{ A_PL2 } & \multirow{3}{*}{100} & $(66.7,166.13)$ & 0.08465 & 0.07839 & 0.08453 & 0.07847 & 50.5 & 165.1 & 0.15 & 0.27 \\
\hline & & $(76.7,176.15)$ & 0.09930 & 0.08082 & 0.09968 & 0.08121 & & & & \\
\hline & & $(56.76,156.27)$ & 0.06982 & 0.07632 & 0.06946 & 0.0758 & & & & \\
\hline
\end{tabular}

Table 4.2 Results for tests of repeatability

\begin{tabular}{|c|c|c|c|c|c|c|c|}
\hline Test code & $\sigma_{c}(k P a)$ & $\left(\Delta \sigma_{h}, \Delta \sigma_{v}\right)(k P a)$ & Sample \# & $M_{r}^{v}(M P a)$ & $M_{r}^{h}(M P a)$ & $v_{h h}$ & $v_{v h}$ \\
\hline \multirow{2}{*}{ A_CTC } & \multirow{2}{*}{100} & \multirow{2}{*}{$(0,100)$} & 1 & 150.9 & - & - & 0.56 \\
\hline & & & 2 & 152.3 & - & - & 0.55 \\
\hline \multirow{2}{*}{ A_PL2 } & \multirow{2}{*}{100} & \multirow{2}{*}{$(66.7,166.7)$} & 1 & 50.5 & 165.1 & 0.15 & 0.27 \\
\hline & & & 2 & 51.6 & 168.5 & 0.15 & 0.27 \\
\hline \multirow{2}{*}{ A_CTC } & \multirow{2}{*}{30} & \multirow{2}{*}{$(0,60)$} & 1 & 93.5 & - & - & 0.81 \\
\hline & & & 2 & 95.2 & - & - & 0.90 \\
\hline \multirow{2}{*}{ A_CTE } & \multirow{2}{*}{50} & \multirow{2}{*}{$(35,0)$} & 1 & 41.6 & 100.2 & 0.14 & 0.21 \\
\hline & & & 2 & 43.8 & 105.3 & 0.13 & 0.20 \\
\hline
\end{tabular}

\subsection{RESILIENT MODULUS}

\subsubsection{Resilient modulus and its dependency on stress}

\section{(a) Stress path with $k \neq 0$}

According to the previous discussion, the initial confining pressure and stress path have significant impact on the resilient modulus, as well as on the development of resilient strains. Figure 4.14 shows the variations of $M_{r}^{v}$ and $M_{r}^{h}$ (the vertical resilient modulus and the horizontal resilient modulus) with respect to the applied $\Delta \sigma_{v}$ for stress paths with 
$k>0$ at initial confining pressures of $30 \mathrm{kPa}$ and $150 \mathrm{kPa}$, respectively ${ }^{1}$. The following are the major findings from the experimental data:

- Along a given stress path, both $M_{r}^{v}$ and $M_{r}^{h}$ increase with an increase in the initial confining pressure.

- For stress paths with $k>0, M_{r}^{v}$ increases with the applied $\Delta \sigma_{v}$. In other words, $M_{r}^{v}$ increases with the overall vertical stress $\sigma_{v}$.

- For a given initial confining pressure, when vertical stress increments $\Delta \sigma_{v}$ are the same, smaller $M_{r}^{v}$ is obtained for stress paths of increased $k$ (corresponds to larger deviator stress). The increase of $M_{r}^{v}$ is small when $k$ varies from 1.5 to 3.0.

- The variation of $M_{r}^{h}$ with $\Delta \sigma_{v}$ also depends on $k$. When $k=1.5, M_{r}^{h}$ is almost constant irrespective any change of $\Delta \sigma_{v}$, whereas for $k=0.5$ and $1.0, M_{r}^{h}$ tends to increase with $\Delta \sigma_{v}$. An opposite trend is observed for the stress path of $k=2$ and 3. In comparison to $M_{r}^{v}$, one clearly observes that the deviator stress has an more substantial influence on $M_{r}^{h}$, particularly for stress paths of increased $k$. It is likely that when $k$ increases $\left(\Delta \sigma_{v} / \Delta \sigma_{h}\right.$ increases), the lateral confinement is not strong enough to resist larger deviator stress, which in turn leads to the development of extensive lateral strain even resilient dilatancy. As such, $M_{r}^{h}$ decreases.

- From a given initial stress state, $M_{r}^{h}$ is consistently lower than $M_{r}^{v}$ for the stress path tests of different $k$ performed in this study.

\footnotetext{
${ }^{1}$ The complete results for all initial pressures employed in this study are shown in Appendix II.
} 

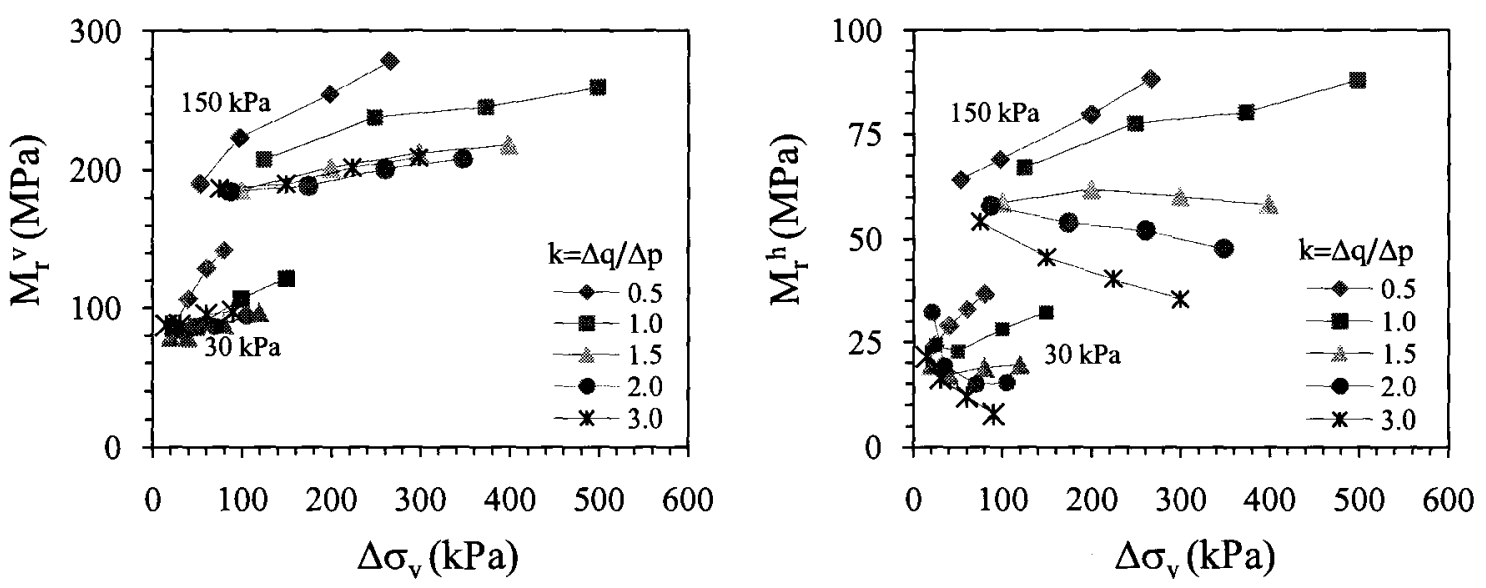

Fig. 4.14 Resilient moduli and stress increments for stress paths $k>0$

Figure 4.15 shows the variations of $M_{r}^{v}$ and $M_{r}^{h}$ with respect to the applied $\Delta \sigma_{h}$ for stress paths having $k<0$. Similar to stress paths with $k>0$, both $M_{r}^{v}$ and $M_{r}^{h}$ increase with an increase in the initial confining pressure. $M_{r}^{h}$ obtained from stress paths with $k<0$ tends to increase slightly with $\Delta \sigma_{h}$, and the dependeny of $M_{r}^{h}$ on $\Delta \sigma_{h}$ appears to be independent of stress path. The influence of $\Delta \sigma_{h}$ on $M_{r}^{v}$ depends on $k$. Specifically, $M_{r}^{v}$ increases with $\Delta \sigma_{h}$ when $k=-0.5$, but decreases with $\Delta \sigma_{h}$ when $k=-1.0$ and -1.5 as the extensive vertical strains occur.

The results lead to the following conclusion: as shown in Fig.1.1, when a rolling wheel moves immediately above a pavement section, the stress path experienced by the granular base element is similar to the positive stress path with a high ratio of $\Delta q / \Delta p$ (close to 3) or $\Delta \sigma_{v} / \Delta \sigma_{h}$. This change in stress conditions can lead to development of extensive lateral strain and a reduction of $M_{r}^{h}$. When a moving wheel is not directly above the granular base element, whether approaching or leaving, the pavement element undergoes a major principal stress rotation that corresponds to stress paths with reduced 
$k$. For stress paths having negative $k$, although $M_{r}^{h}$ increases, the low ratio of $\Delta q / \Delta p$ or $\Delta \sigma_{v} / \Delta \sigma_{h}$ may result in a drop of $M_{r}^{v}$. The reduced $M_{r}^{v}$ and $M_{r}^{h}$ of granular base could lead to a situation where less than assumed support for the upper AC layer and underlying subgrade develops. Therefore, the selection of $M_{r}^{v}$ and $M_{r}^{h}$ is critical for realistic flexible pavement design.
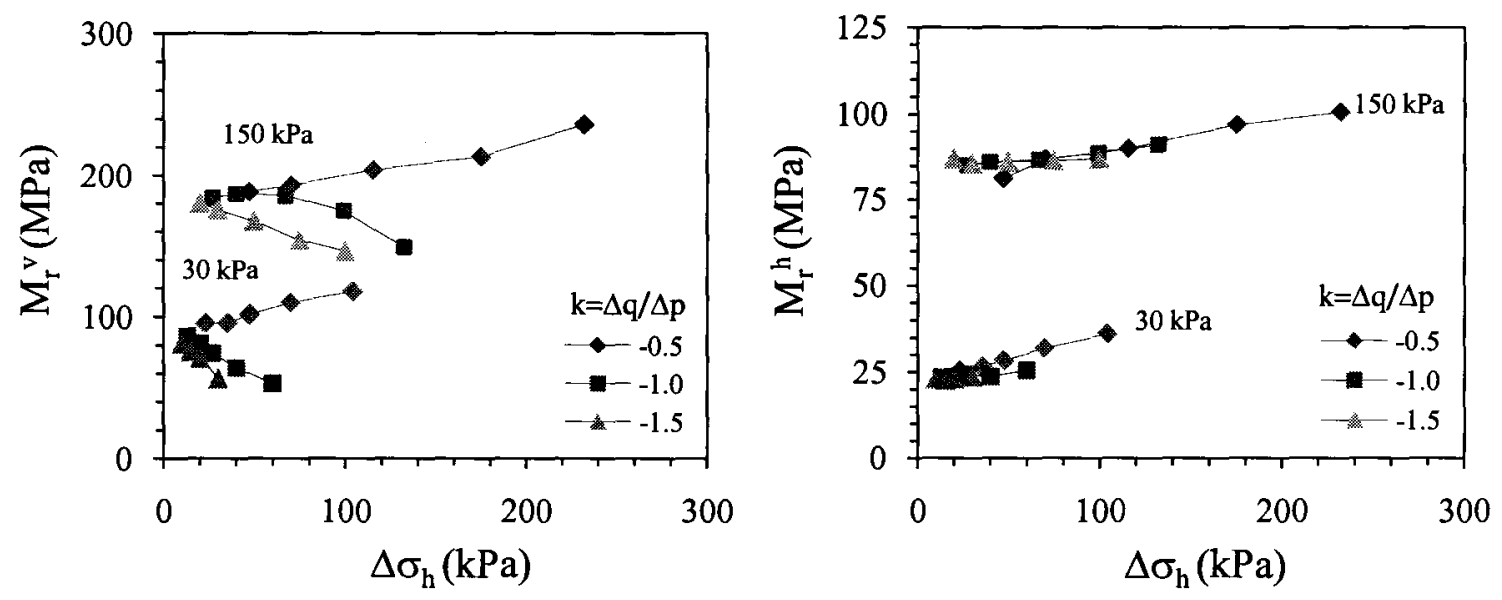

Fig. 4.15 Resilient moduli and stress increments for stress paths $k<0$

\section{(b) IC loading path}

Figure 4.16 shows that variations of $M_{r}^{v}$ and $M_{r}^{h}$ with applied stresses for IC loading paths. As outlined previously, the material is contractive in both directions during loading, with $M_{r}^{v}$ and $M_{r}^{h}$ increasing with the initial confining pressures $\sigma_{c}$ and the peak stress $\sigma_{c}^{\prime}\left(=\sigma_{c}+\Delta \sigma_{c}\right)$ in each stress cycle. Similar to stress paths having $k \neq 0, M_{r}^{h}$ is consistently lower than $M_{r}^{v}$ within the framework of this study. The significant differences between $M_{r}^{v}$ and $M_{r}^{h}$ can be attributed to the inherent fabric. It can be seen that both $M_{r}^{v}$ and $M_{r}^{h}$ can be expressed as power functions of the mean effective stress 
$p\left(=\sigma_{c}^{\prime}\right)$, but with different regression constants.

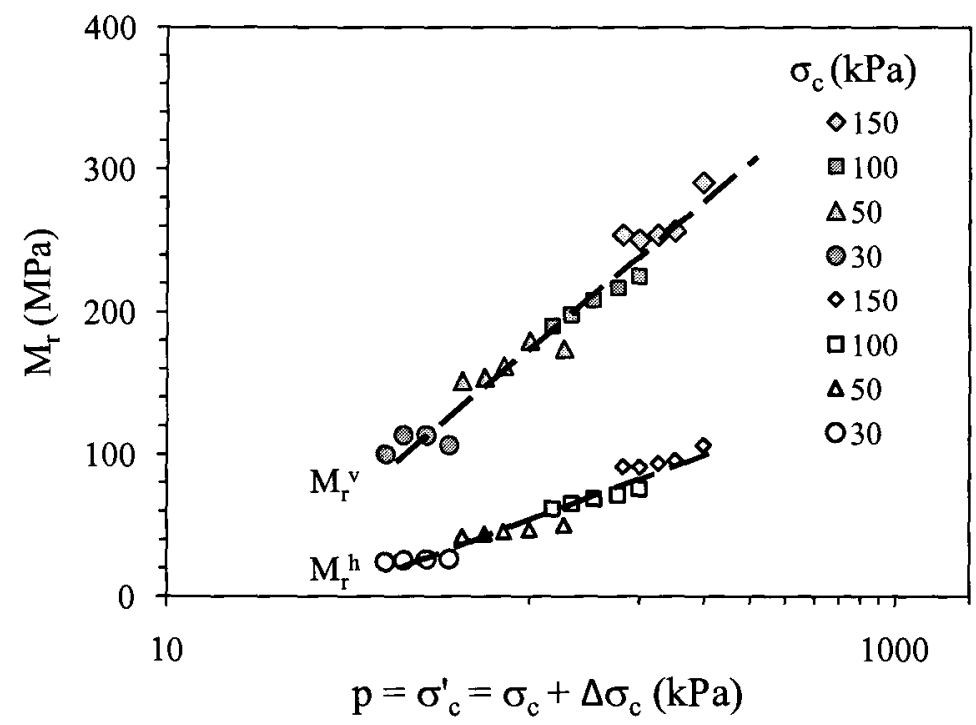

Fig. 4.16 Resilient moduli and stress increments for IC compression

\section{(c) Validity of the Uzan and AASHTO resilient modulus equations}

Figures 4.14 through 4.16 clearly show that $M_{r}^{v}$ and $M_{r}^{h}$ depend on both the initial stress state and the stress path. Therefore, the characterization of resilient modulus simply based on final stress state (i.e., state which corresponds to the peak stress during cyclic loading), such as the Uzan and AASHTO resilient modulus equations, may be considered inadequate and incomplete for describing complex resilient properties of granular materials along general stress paths. Figures 4.17 and 4.18 show the variations of $M_{r}^{v}$ and $M_{r}^{h}$ as a function of the mean effective stress $p(=\theta / 3)$ and the deviator stress $\sigma_{d}\left(=\sigma_{1}-\right.$ $\left.\sigma_{3}\right)$. It can be seen from Fig. 4.17 that for stress paths with positive $k, M_{r}^{v}$ increases with $p$ and $\sigma_{d}$, but the extent of dependency on $p$ and $\sigma_{d}$ depends on $k$. More specifically, $M_{r}^{v}$ tends to increase more with $p$ and $\sigma_{d}$ when $k$ is small. Figure 4.18 shows that $M_{r}^{h}$ may 
either increase or decrease with $p$ and $\sigma_{d}$, depending on the stress path along which the test is performed. For example, for stress paths of $k=0.5,1.0$ and 1.5, the horizontal resilient deformation is compressive, $M_{r}^{h}$ increases with applied stresses; when $k$ is increased to 2.0 and $3.0, M_{r}^{h}$ decreases with $p$ and $\sigma_{d}$. This is attributed to higher $k$ values inducing larger deviator stresses during loading, which in turn causes resilient dilation with horizontal extensive resilient strain developed and smaller values of $M_{r}^{h}$.
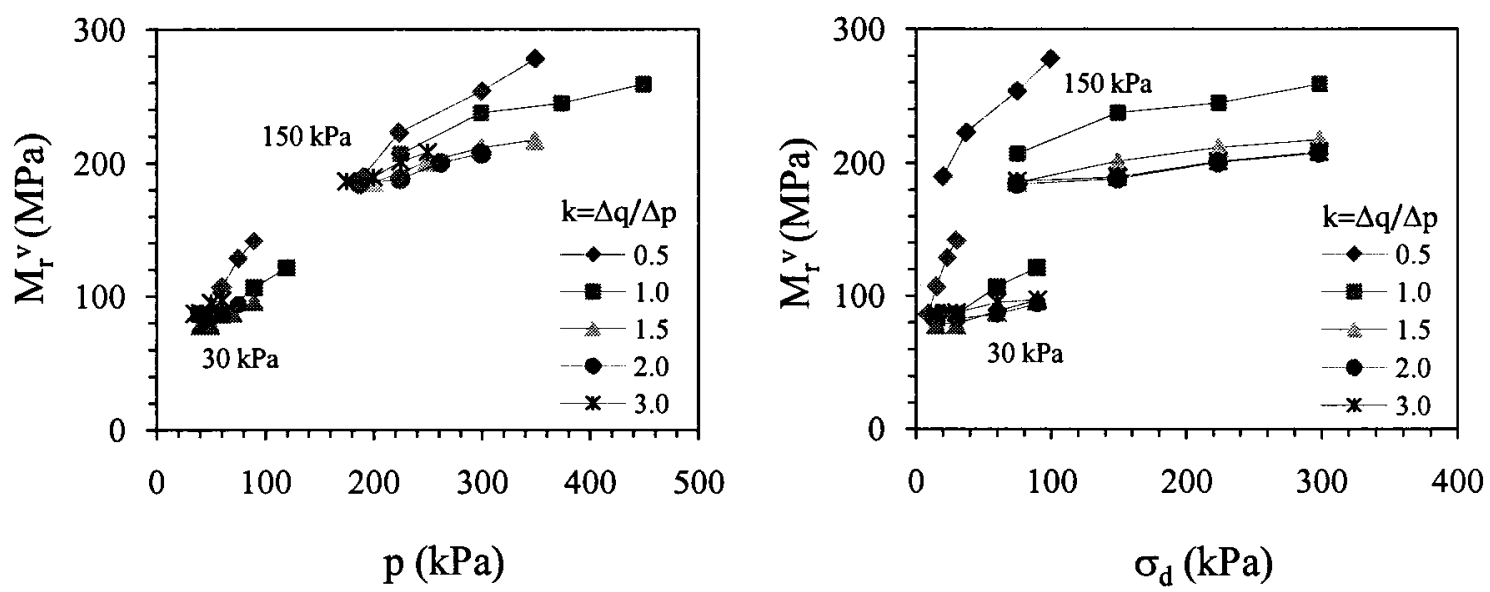

Fig. 4.17 Vertical resilient moduli and stress increments for stress paths $k>0$
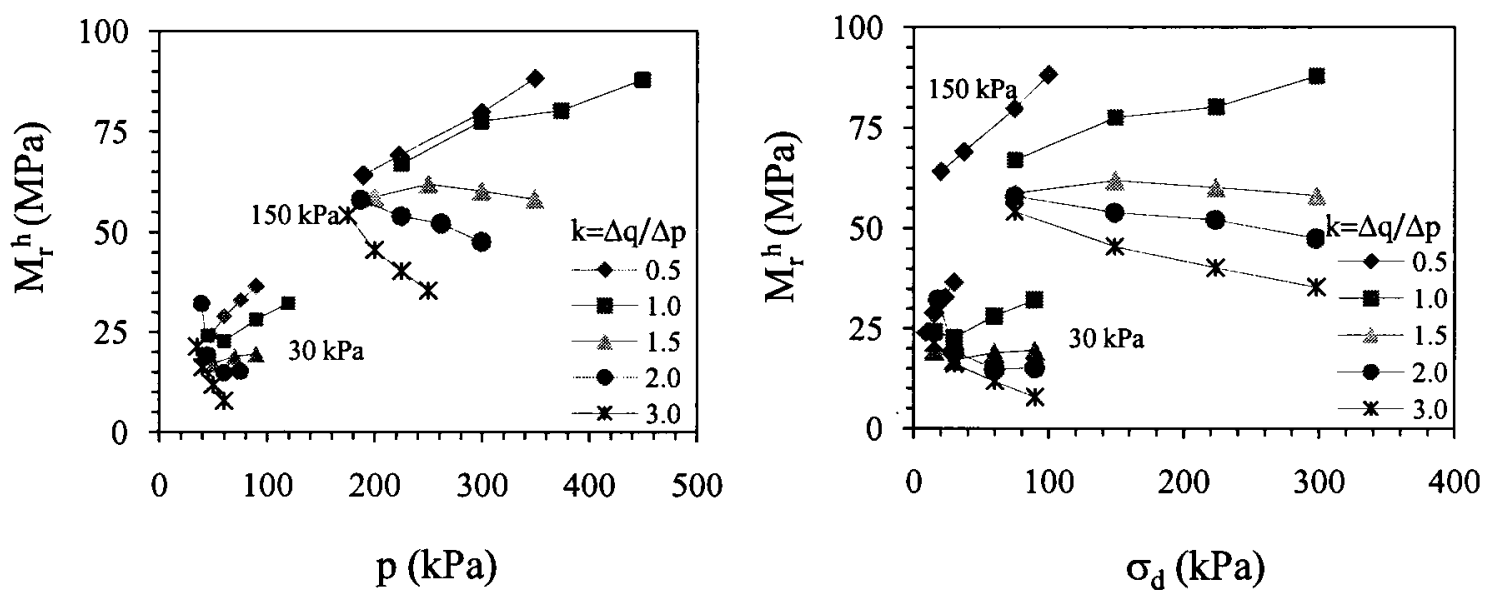

Fig. 4.18 Horizontal resilient moduli and stress increments for stress paths $k>0$ 
Similar to $M_{r}^{h}$ obtained from positive $k$ stress paths, $M_{r}^{v}$ obtained from negative $k$ stress paths may either increase or decrease with $p$ and $\sigma_{d}$, depending on the applied stress paths, along which $\varepsilon_{v, r}$ may either be compressive or extensive, see Fig. 4.19.
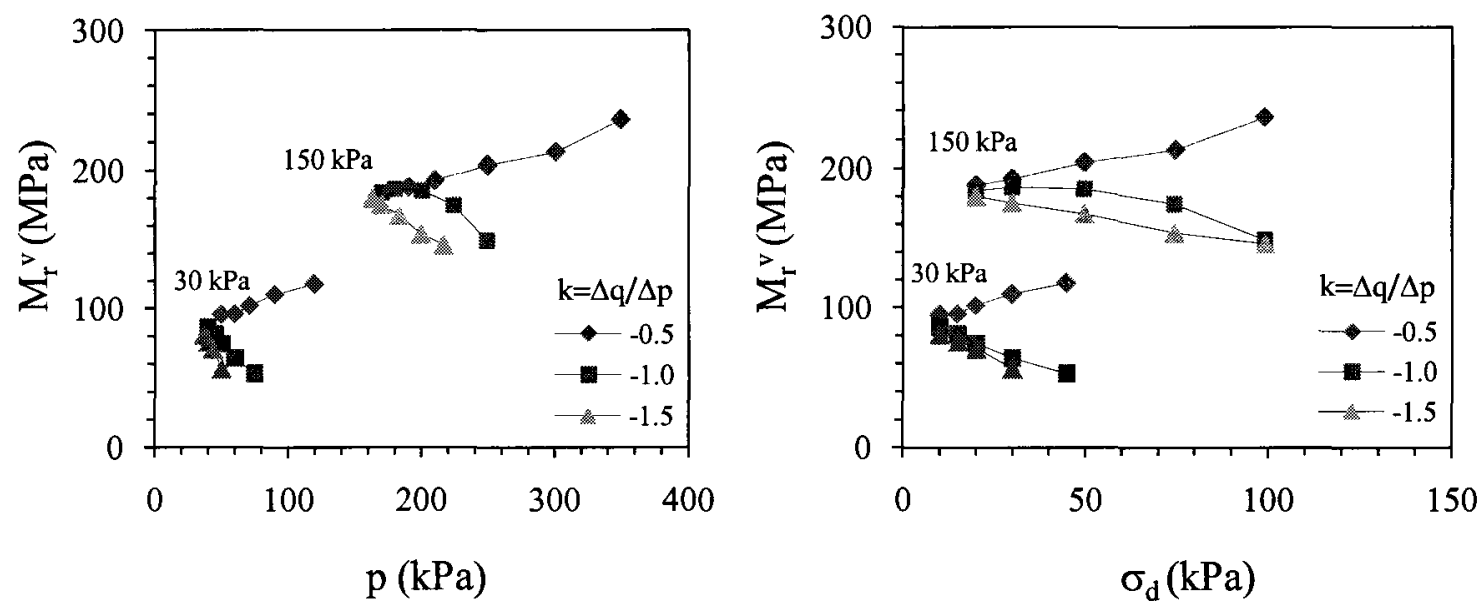

Fig. 4.19 Vertical resilient moduli and stresses for stress paths $k<0$

As shown in Fig. 4.20, $M_{r}^{h}$ obtained from negative $k$ stress paths is found to increase with $p$, however, higher initial confining pressures yield higher $M_{r}^{h}$ for the same final mean stress $p$. In addition, $M_{r}^{h}$ also increases with $\sigma_{d}$, but the dependency on $\sigma_{d}$ is slightly different for each negative stress path.

According to Figs 4.17 through 4.20, one concludes that the dependency of resilient modulus, either $M_{r}^{h}$ and $M_{r}^{v}$, on $p$ and $\sigma_{d}$ varies with stress path. In other words, the coefficients associated with $\theta$ and $\sigma_{d}$ in the Uzan and AASHTO resilient modulus equations depend on stress path and are not fixed values. Therefore, one may argue that the Uzan and AASHTO resilient modulus equations, which are based on classical cyclic triaxial tests with constant confining pressure, are not appropriate for the characterization 
of resilient modulus for various stress paths. Equations, which are based on standard resilient modulus tests, tend to over-estimate $M_{r}^{h}$ and $M_{r}^{v}$ for stress paths with $k<0$. Caution must therefore be exercised when using these equations.
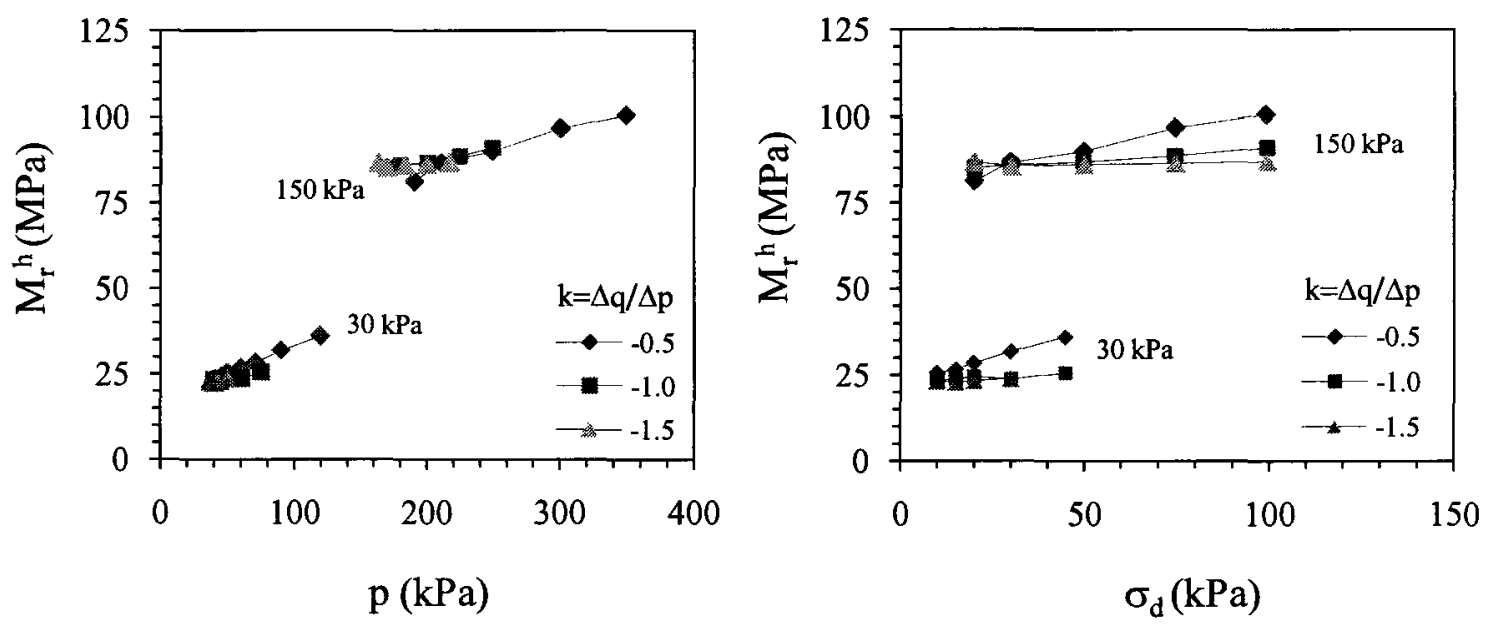

Fig. 4.20 Horizontal resilient moduli and stresses for stress paths $k<0$

\subsubsection{Resilient modulus and its dependency on resilient strain}

\section{(a) Stress path with $k \neq 0$}

As discussed previously, the dependency of $M_{r}^{v}$ and $M_{r}^{h}$ on applied stress varies with the stress path, due to the fact that various ratios of $\Delta \sigma_{v} / \Delta \sigma_{h}$ lead to different resilient deformation characteristics. This section however focuses on the relations between resilient modulus and resilient strain for various stress paths.

Figure 4.21 presents the variations of $M_{r}^{v}$ and $M_{r}^{h}$ with $\varepsilon_{h, r}$ and $\varepsilon_{v, r}$ for stress paths with $k>0$ for initial $\sigma_{c}$ of $30 \mathrm{kPa}$ and $150 \mathrm{kPa}$, respectively ${ }^{2}$. Depending on the values of

\footnotetext{
${ }^{2}$ The complete results for all initial pressures employed in this study are shown in Appendix II.
} 
$k$, three deformation patterns are observed for this material: (a) $\varepsilon_{v, r}>0$ and $\varepsilon_{h, r}>0$ when $k=0.5 \sim 1.5$; (b) $\varepsilon_{v, r}>0$ and $\varepsilon_{h, r} \sim 0.001 \%$ for $k=2$; and (c) $\varepsilon_{v, r}>0$ and $\varepsilon_{h, r}<0$ for $k=3$. This deformation pattern is closely related to the shear stress level, or resilient dilatancy in the tests. Moreover, for a given stress path, the relations of $M_{r}^{v}$ vs $\varepsilon_{v, r}, M_{r}^{v} v S \varepsilon_{h, r}, M_{r}^{h} v s \varepsilon_{v, r}$ and $M_{r}^{h} v S \varepsilon_{h, r}$ vary with initial $\sigma_{c}$; i.e., both $M_{r}^{v}$ and $M_{r}^{h}$ increase with an increase in $\sigma_{c}$ given the same resilient strain.

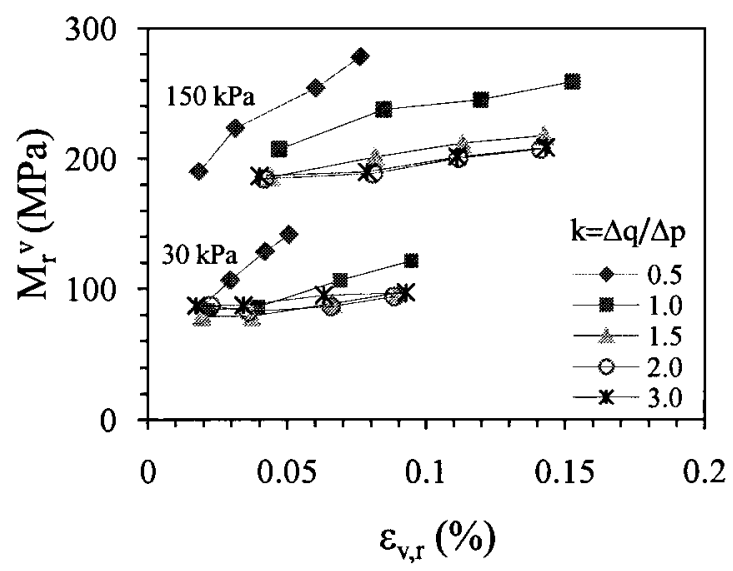

(a)

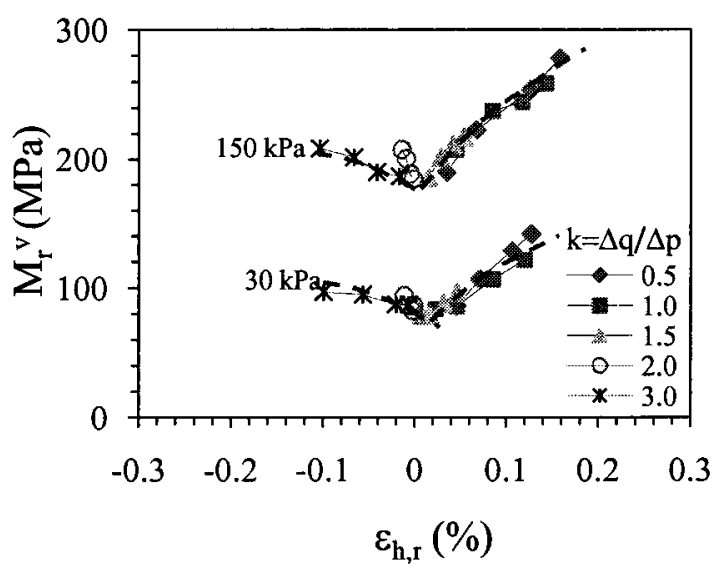

(c)

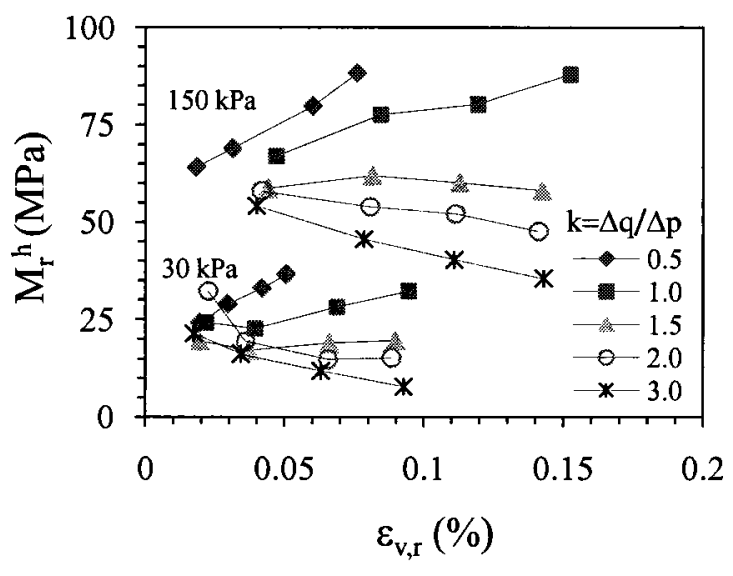

(b)

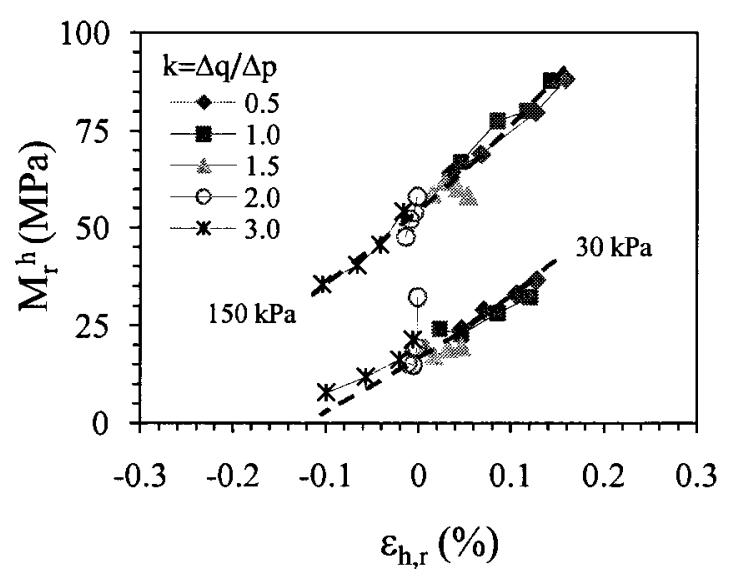

(d)

Fig. 4.21 Resilient moduli and resilient strain components for stress paths $k>0$ 
As can be seen from Figs. 4.21(a) and (b), the dependency of resilient modulus, either $M_{r}^{v}$ or $M_{r}^{h}$, on $\varepsilon_{v, r}$ depends on $k$ given constant $\sigma_{c}$. Specifically, $M_{r}^{v}$ increases as $\varepsilon_{v, r}$ increases, with $M_{r}^{v}$ increasing more rapidly when $k$ decreases. $M_{r}^{h}$, on the hand, increases with $\varepsilon_{v, r}$ when $\varepsilon_{h, r}>0$ for $k=0.5 \sim 1.5$, but decreases with $\varepsilon_{v, r}$ when $\varepsilon_{h, r}<0$ for $k=2 \sim 3$.

For stress path tests with $k>0$, one observes that $\varepsilon_{h, r}$ has an important influence on $M_{r}^{v}$ and $M_{r}^{h}$. As shown in Fig. 4.21(c), the dependency of $M_{r}^{v}$ on $\varepsilon_{h, r}$ varies with the sign of $\varepsilon_{h, r}$; i.e., compressive (+) or extensive (-). For example, for $k=0.5 \sim 1.5$, given constant $\sigma_{c}, M_{r}^{v}$ tends to increase with $\varepsilon_{h, r}$ when $\varepsilon_{h, r}>0$. For $k=3.0$, When $\varepsilon_{h, r}<0$, $M_{r}^{v}$ increases as $\varepsilon_{h, r}$ decreases but to a lesser degree when compared to stress paths with $k=0.5 \sim 1.5$. The trend for $M_{r}^{v}$ variation for $k=2.0$, however, seems to be different from the others. This is attributed to the fact that the level of $\varepsilon_{h, r}$ developed is very small $(\sim 0.001 \%)$ compared to $\varepsilon_{v, r}$, which in turn could lead to round off errors.

It is noted that within the range of the stresses in this study, the phenomenon that $M_{r}^{v}$ decreases with extensive $\varepsilon_{h, r}$ (corresponding to higher deviator stress) was not observed. However, in reality, when the repeated deviator stress is greater than a threshold, such as the deviator stress at failure proposed by Van Niekerk (2002), the material may display stress/strain softening, therefore, it is very likely that $M_{r}^{v}$ first increases with extensive $\varepsilon_{h, r}$ then decreases when the magnitude of extensive $\varepsilon_{h, r}$ becomes larger as resilient dilatancy becomes greater.

Fig. 4.21(d) shows that the dependency of $M_{r}^{h}$ on $\varepsilon_{h, r}$ is relatively independent of 
the stress path, with $M_{r}^{h}$ consistently increasing as $\varepsilon_{h, r}$ increases. When extensive $\varepsilon_{h, r}$ develops, $M_{r}^{h}$ decreases and can be very low especially at a low confining pressure.

For stress paths with $k<0$, the major principal stress is in the horizontal direction, the specimen is found to develop compressive horizontal strain and possible compressive, extensive or very small vertical strain. The dependency of $M_{r}^{v}$ and $M_{r}^{h}$, on resilient strains is well defined in Fig. 4.22. The influence of initial confining pressure on the relations of resilient moduli and resilient strains is consistent with those having $k>0$. One observes from Figs. 4.22(a) and (d) that the dependencies of $M_{r}^{v}$ on $\varepsilon_{v, r}$ and $M_{r}^{h}$ on $\varepsilon_{h, r}$ are independent of stress path, with $M_{r}^{v}$ consistently increasing with $\varepsilon_{v, r}$, and $M_{r}^{h}$ consistently increasing with $\varepsilon_{h, r}$. However, dependencies of $M_{r}^{v}$ on $\varepsilon_{h, r}$ and $M_{r}^{h}$ on $\varepsilon_{v, r}$ vary with stress path. As shown in Fig. 4.22(b), when $\varepsilon_{v, r}>0, M_{r}^{h}$ increases with an increase in $\varepsilon_{v, r}$. When $\varepsilon_{v, r}<0, M_{r}^{h}$ also increases as extensive $\varepsilon_{v, r}$ decreases, but to a lesser degree in comparison to those having compressive $\varepsilon_{v, r}$. Similar to $M_{r}^{v}$ for stress paths with $k>$ $0, M_{r}^{h}$ for stress paths with $k<0$ may also exhibit stress/strain softening when the magnitude of extensive $\varepsilon_{v, r}$ becomes larger or resilient dilatancy becomes greater. The variation of $M_{r}^{v}$ with $\varepsilon_{h, r}$ is presented in Fig. 4.22(c). When $\varepsilon_{v, r}>0, M_{r}^{v}$ increases with $\varepsilon_{h, r}$ for $k=-0.5$. When $\varepsilon_{v, r}<0, M_{r}^{v}$ decreases with $\varepsilon_{h, r}$ for $k=-1.0$ and -1.5 .

As such, one concludes that the resilient modulus $M_{r}^{\text {minor }}$ in the direction of minor principal stress, tends to increase monotonically with the resilient strain $\varepsilon_{r}^{\text {minor }}$ in the same direction. However, $M_{r}^{\text {major }}$ tends to increase with the amplitude of $\left|\varepsilon_{r}^{\text {minor }}\right|$, implying either compression or extension in the direction of minor principal stress causes 
increased $M_{r}^{\text {major }}$, which is likely the coupled effects of stress level and dilation. In other words, $M_{r}^{v}$ can be expressed as a function of $\varepsilon_{r}^{\text {minor }}$. While $M_{r}^{h}$ can be expressed as a function of $\varepsilon_{h, r}$. The relations of $M_{r}^{v} v s \varepsilon_{r}^{\text {minor }}$ for $\varepsilon_{r}^{\text {minor }}>0$ and $\varepsilon_{r}^{\text {minor }}<0$, and $M_{r}^{h} v s \varepsilon_{h, r}$ for $k>0$ and $k<0$ are independent of $k$, respectively. The differences in the dependency on resilient strain between $M_{r}^{v}$ and $M_{r}^{h}$ may be attributed to the soil fabric or bedding plane orientation, which needs further investigation.

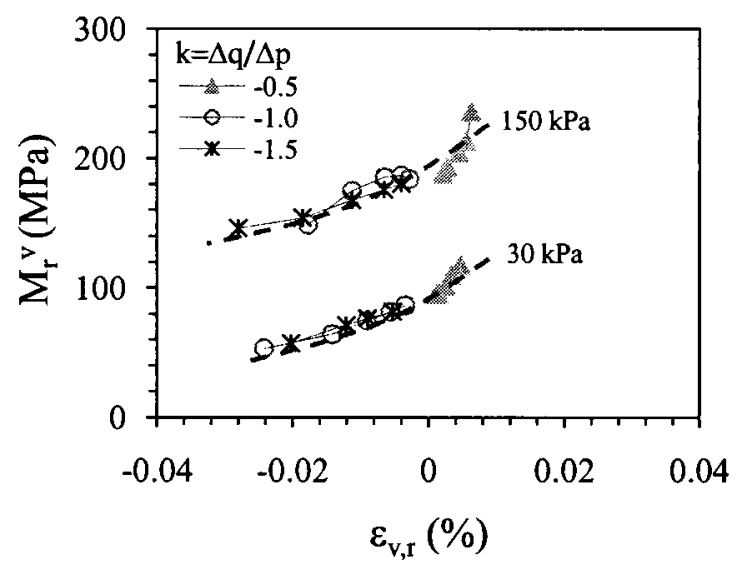

(a)

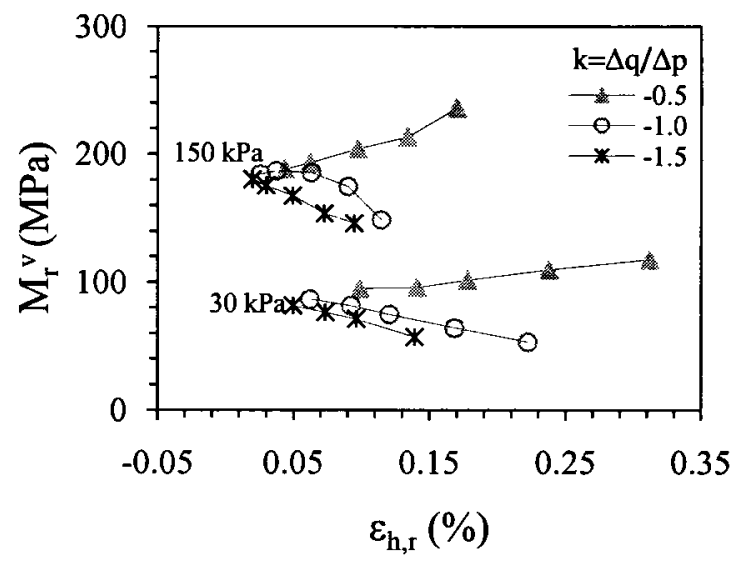

(c)

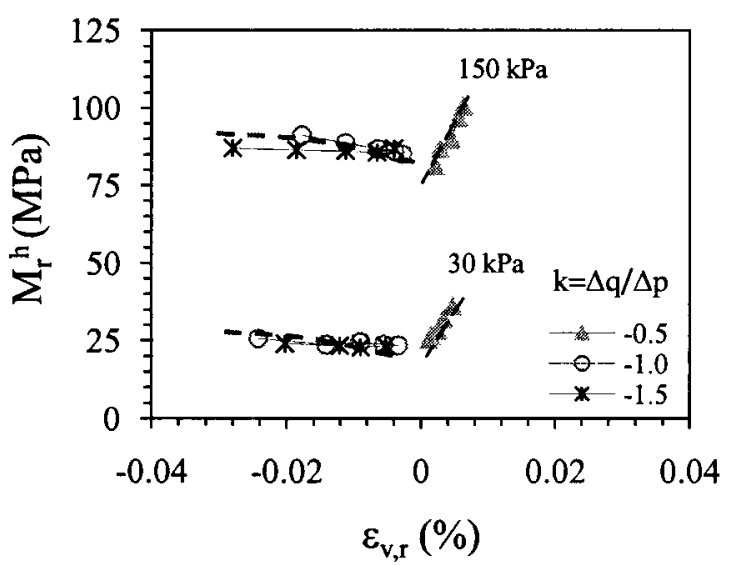

(b)

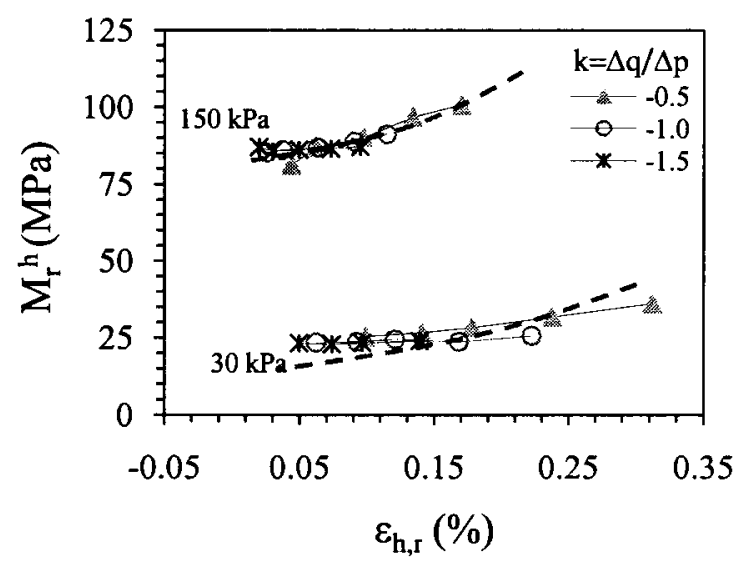

(d)

Fig. 4.22 Resilient moduli and resilient strain components for stress paths $k<0$ 
Scrutinizing the experimental data reveals that the strain dependency of resilient modulus in this study is not consistent with the general understanding on the strain level dependency of modulus. According to the shear modulus reduction curve for most geomaterials, when the shear stress-induced shear strain is at the level of $0.0001 \%$, the material can be considered as linear elastic, which corresponds to the maximum shear modulus. When the strain level is larger than $0.001 \%$, the shear modulus decreases with an increase in the shear strain level. However, the strain level within the framework of this research ranges from $0 \sim 0.5 \%$. The dependency of the resilient modulus, either $M_{r}^{v}$ or $M_{r}^{h}$ on resilient strains varies with direction and stress path.

\section{(b) IC loading path}

For the IC loading path, the specimen is loaded hydrostatically. As shown in Fig. 4.11, the ratio of $\varepsilon_{h, r} / \varepsilon_{v, r}$ decreases as the initial confining pressure $\sigma_{c}$ increases, indicating that the deformation anisotropy decreases. Fig. 4.23 shows that both $M_{r}^{v}$ and $M_{r}^{h}$ increase as $\sigma_{c}$ and $\varepsilon_{h, r}$ increase, but the effect of $\sigma_{c}$ is more significant, implying that the subsequent stress changes has less influence on the increase in the resilient modulus, at least for the conditions investigated in this research. As $\varepsilon_{v, r} / \varepsilon_{h, r}$ is almost constant for a given $\sigma_{c}$, both $M_{r}^{v}$ and $M_{r}^{h}$ can be expressed as functions of $\varepsilon_{v, r}$ or $\varepsilon_{h, r}$ in addition to $\sigma_{c}$. 


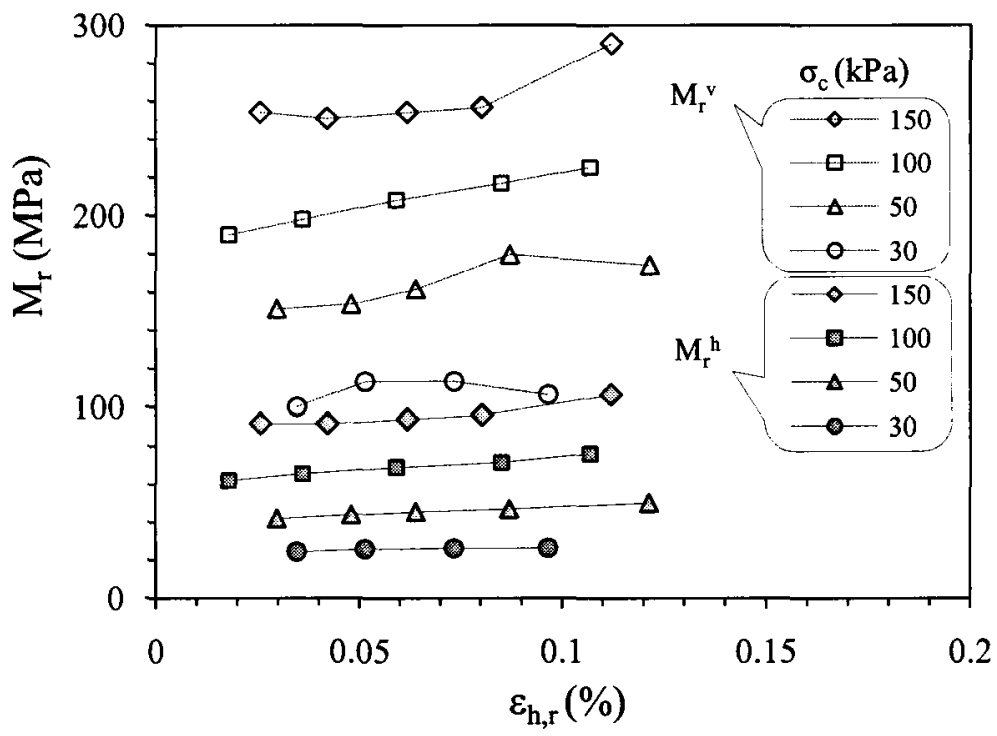

Fig. 4.23 Stress and strain components for IC loading paths

\subsection{RESILIENT POISSON'S RATIO AND STRESS}

The use of constant Poisson's ratio for geomaterials is common practice. However, the experimental results in this study reveal that the resilient Poisson's ratio is not constant, but depends on direction and stress path. The relation between Poisson's ratio and stress appears to be quite complex as shown by the summary of results presented in Fig. 4.24.

For stress paths with $k>0$, as shown in Figs. 4.24(a) and (b), there is a systematic change in the way the Poisson's ratio vary with $\sigma_{v} / \sigma_{h}$ as the stress path changes from $k=0.5 \sim 3.0$. Given constant $\sigma_{v} / \sigma_{h}$, both $v_{v h}$ and $v_{h h}$ increase as $k$ increases. When $k=0.5$ and 1.0, $v_{v h}$ and $v_{h h}$ decrease as $\sigma_{v} / \sigma_{h}$ increases. When $k=1.5, v_{v h}$ and $v_{h h}$ seem to be independent of $\sigma_{v} / \sigma_{h}$, and can be considered as constant. However, when the extensive $\varepsilon_{h, r}$ occurs for $k=2.0$ and 3.0 , both $v_{v h}$ and $v_{h h}$ increases as $\sigma_{v} / \sigma_{h}$ increases, 
and $v_{v h}$ can even exceed 0.5 when resilient dilatancy occurs.

In comparison to stress paths with $k>0$, the variations of $v_{v h}$ and $v_{h h}$ with $\sigma_{v} / \sigma_{h}$ for stress paths with $k<0$ are relatively small and can be considered as constant., see Fig. 4.24(c). Figure 4.24 (d) presents the variations of $v_{v h}$ and $v_{h h}$ with $\sigma_{c}^{\prime} / \sigma_{c}$ for the IC loading path. In general, given constant $\sigma_{c}$, one observes that both $v_{v h}$ and $v_{h h}$ slightly decrease as $\sigma_{c}^{\prime} / \sigma_{c}$ increases, but the variations are small. The trends of variations are similar to those for stress paths with $k=0.5$ and 1.0. This might be attributed to the constraints on deformation as the specimen is densely compacted in both the vertical and the horizontal directions, which leads to reductions of $v_{v h}$ and $v_{h h}$. These results are in a good agreement with the findings reported by Chaudhary et.al. (2004) from IC tests and HCA tests with $k=0.5$ on dense Toyoura sand.

Overall, $v_{v h}$ and $v_{h h}$ do not appear to be too sensitive to the confining pressure with the stress range studied. The value of $v_{h h}$ appears to be smaller than $v_{v h}$ for all stress path tests. More specifically, $v_{v h}$ may vary over a wider range of $0.02 \sim 1.1$ depending on the $k$ value, while $v_{h h}$ is generally in the range of $0.01 \sim 0.3$. For specimens subjected to hydrostatic compression, both $v_{v h}$ and $v_{h h}$ vary in relatively small ranges with typical values being $v_{v h}=0.2 \sim 0.25$ and $v_{h h}=0.1 \sim 0.2$. It is suggested that the higher value of $v_{v h}$ reflects anisotropy of the material and resilient dilatancy induced by large shear stress during the test. The value of $v_{v h}$ can exceed 0.5 and may even be greater than 1 . As the dilatant behaviour of granular materials for pavement base layer cannot be neglected, selection of $v_{v h}$ and $v_{h h}$ for mechanical analysis of pavement must be prudent. 


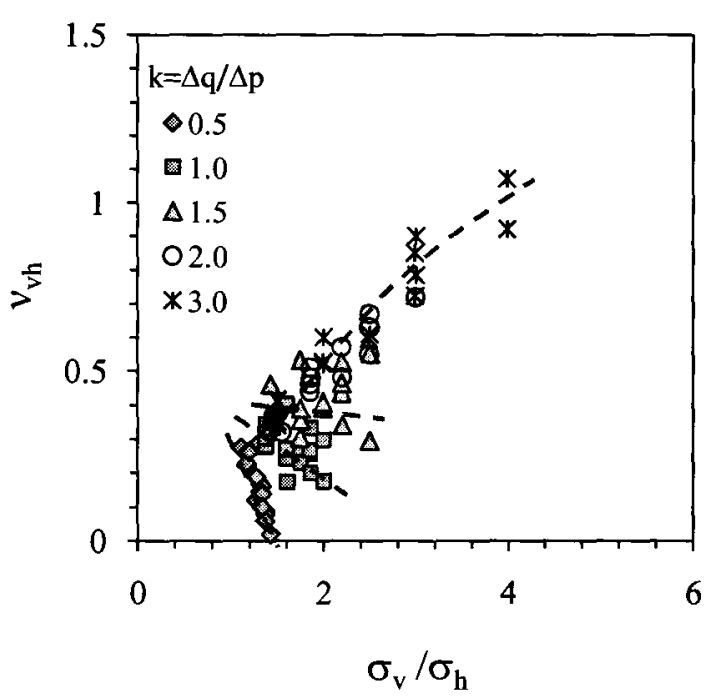

(a)

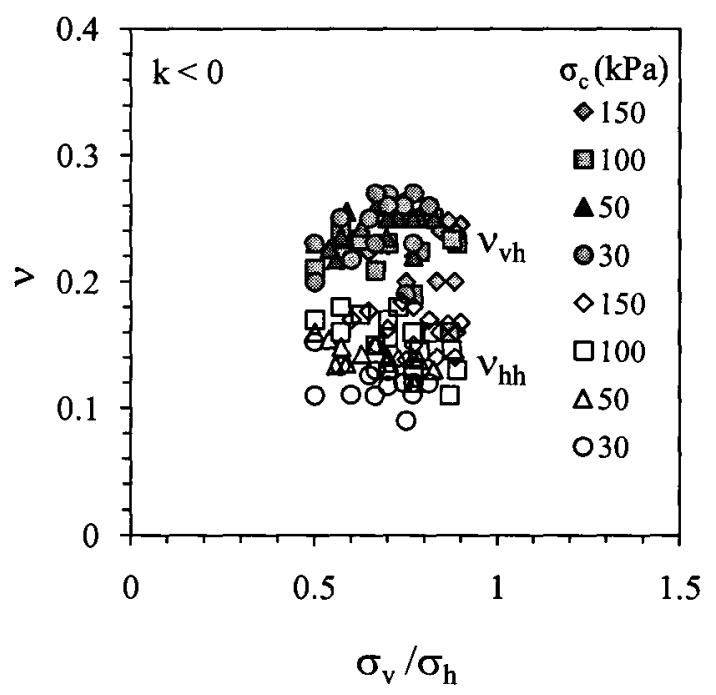

(c)

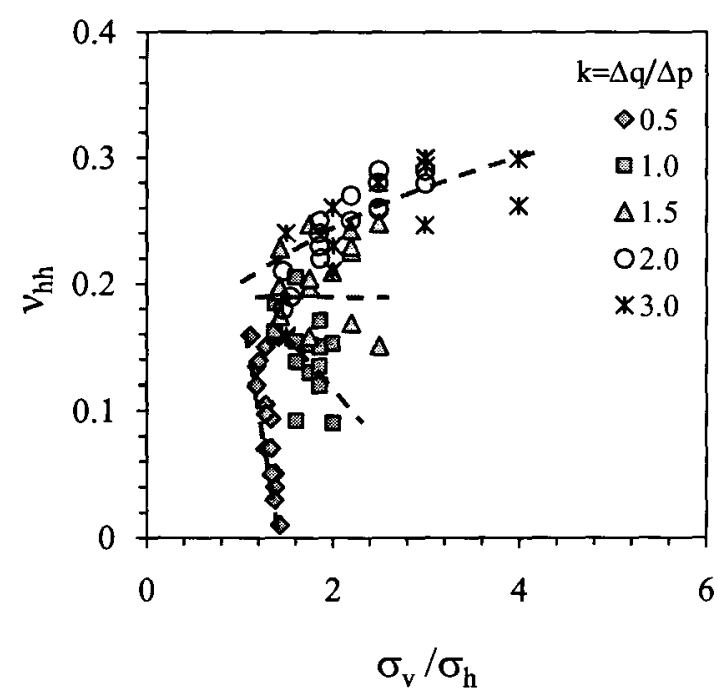

(b)

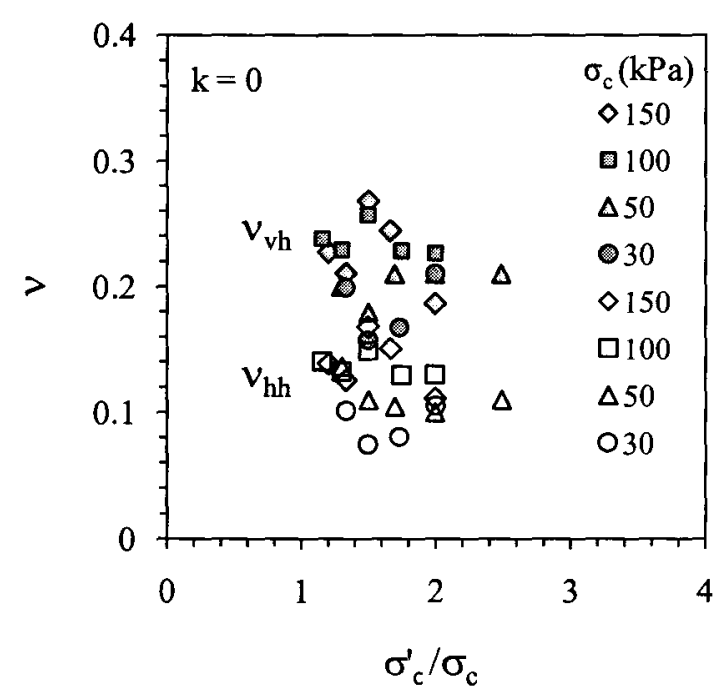

(d)

Fig. 4.24 Poisson's ratio and stresses 


\subsection{STIFFNESS ANISOTROPY}

\subsubsection{Stiffness anisotropy and deformation}

As the stiffness anisotropy can be quantified by $\left(1-M_{r}^{h} / M_{r}^{v}\right)$, it is explored next by examining the modulus ratio of $M_{r}^{h} / M_{r}^{v}$ for each stress path. For isotropic materials, one expects $M_{r}^{h} / M_{r}^{v}=1$. A value of $M_{r}^{h} / M_{r}^{v}$ lower than unity stands for stronger fabric in the vertical direction than that in the horizontal direction.

Figure 4.25 shows that relations between $M_{r}^{h} / M_{r}^{v}$ and $\varepsilon_{v o l, r} / \varepsilon_{v, r}$ (for $k \geq 0$ ) or $\varepsilon_{v o l, r} / 2 \varepsilon_{h, r}$ (for $k \leq 0$ ) for different strain paths. It can be seen that the stiffness anisotropy depends on the stress path along which stress increments are applied to measure the resilient deformation. For stress paths with $k>0$, when extensive $\varepsilon_{h, r}$ develops, the modulus ratio $M_{r}^{h} / M_{r}^{v}$ drops rapidly, and can be as low as 0.05 for $k=2.0$ and 3.0, see Fig. 4.25(a). For other stress paths with $k>0$, although $\sigma_{v} / \sigma_{h}>1$, the deviator stress are relatively lower than that of increased $k$, hence $M_{r}^{h} / M_{r}^{v}$ decreases slower.

For the IC loading path, as discussed already, for a given $\sigma_{c}$, both $M_{r}^{h}$ and $M_{r}^{v}$ increase as stress increases, but $\varepsilon_{h, r} / \varepsilon_{v, r}$ almost remains constant, see Figs. 4.23 and 4.11, which implies changes in fabric are very small. It can be seen from Figs. 4.25(a) and (b) that at a given $\sigma_{c}$, changes in the stiffness anisotropy for the IC loading path are also very small and can be considered as constant. Therefore, there must be something behind that links the fabric and stiffness anisotropy, which need further investigation. On the other hand, the stiffness anisotropy decreases as $\sigma_{c}$ increases. 


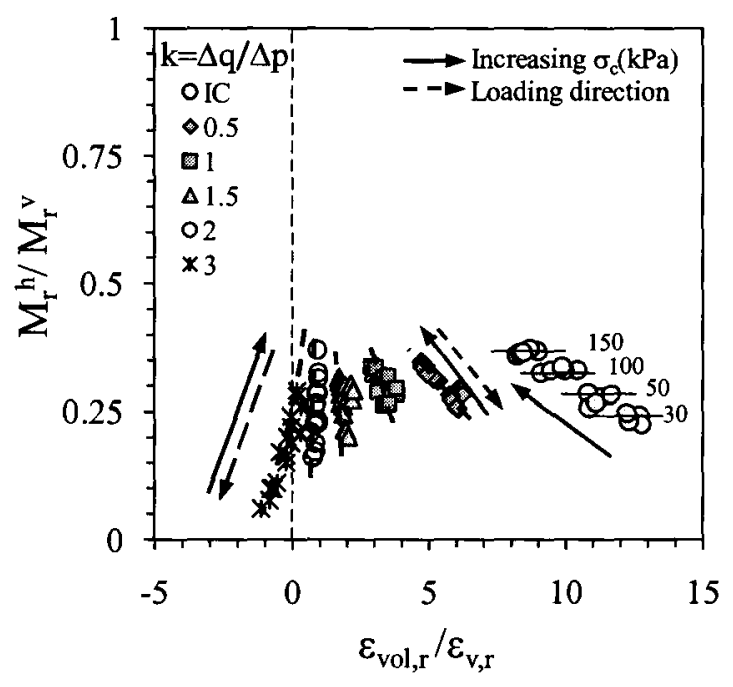

(a)

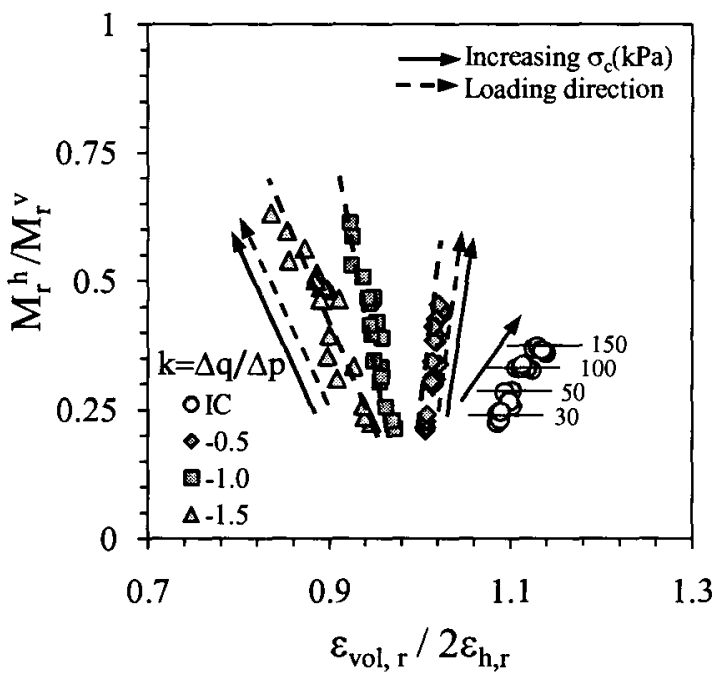

(b)

Fig. 4.25 Stiffness anisotropy and deformation characteristics

For stress paths with $k<0, \frac{\sigma_{v}}{\sigma_{h}}<1$, the material appears to become less anisotropic along the loading path; as shown by the evaluation of $M_{r}^{h} / M_{r}^{v}$ in Fig. 4.25(b). When the material is compacted in both the vertical and horizontal directions, as discussed in previous sections, both $M_{r}^{h}$ and $M_{r}^{v}$ increases along the loading path (e.g. $k=-0.5$ ), but $M_{r}^{h}$ increases more rapidly than $M_{r}^{v}$, thus $M_{r}^{h} / M_{r}^{v}$ increases. When extensive $\varepsilon_{v, r}$ develops for $k=-1.0$ and $-1.5, M_{r}^{h} / M_{r}^{v}$ increases as $M_{r}^{h}$ increases and $M_{r}^{v}$ decreases.

Although the variation of stiffness anisotropy for each stress path varies, the stiffness anisotropy for all stress paths is reduced when confining pressure increases; as illustrated by the solid arrows that show the direction of increasing initial confining pressures in Fig. 4.25. Another conclusion that can be made is that the $M_{r}^{h} / M_{r}^{v}$ may not be directly related to the resilient strain ratio, since $M_{r}^{h} / M_{r}^{v}$ may either increase or 
decrease when the resilient strain ratio increases.

\subsubsection{Stiffness anisotropy and stress}

\section{(a) Stress-induced stiffness anisotropy}

As indicated already, $M_{r}^{h} / M_{r}^{v}$ cannot be directly related to resilient strain ratio. In this section, the stiffness anisotropy, quantified by $\left(1-M_{r}^{h} / M_{r}^{v}\right)$, is examined to find its relation with the applied stresses.

Figure 4.26(a) shows the variation of $M_{r}^{h} / M_{r}^{v}$ with $\sigma_{v} / \sigma_{h}$ at $\sigma_{c}=50 \mathrm{kPa}$ in this study ${ }^{3}$. It can be seen that for some stress paths with $k=-0.5 \sim 1.0$ that corresponds to lower deviator stress levels, the material is contractive in both horizontal and vertical directions, the ratios of $M_{r}^{h} / M_{r}^{v}$ change within a small range. For other stress paths, such as stress paths with $k=1.5 \sim 3$ or $k \leq-1.0$, a relation between $M_{r}^{h} / M_{r}^{v}$ and $\sigma_{v} / \sigma_{h}$ appears to be well defined. More specifically, $M_{r}^{h} / M_{r}^{v}$ decreases when $\sigma_{v} / \sigma_{h}$ is increased. The small variation of $M_{r}^{h} / M_{r}^{v}$ when $k=-0.5 \sim 1.0$, may imply that the inherent anisotropy dominates the material's performance at relatively lower deviator stress level, and $\sigma_{v} / \sigma_{h}$ has a secondary effect on the stiffness anisotropy. At increased (or decreased) $\sigma_{v} / \sigma_{h}$ ratios or $k$ values, the stiffness anisotropy tends to be governed by stress-induced anisotropy, which results in a well-defined relation between $M_{r}^{h} / M_{r}^{v}$ and $\sigma_{v} / \sigma_{h}$; i.e., $M_{r}^{h} / M_{r}^{v} \propto\left(\sigma_{v} / \sigma_{h}\right)^{m_{1}}$, where $m_{1}$ is a regression constant.

${ }^{3}$ The complete results for all initial pressures employed in this study are shown in Appendix II. 


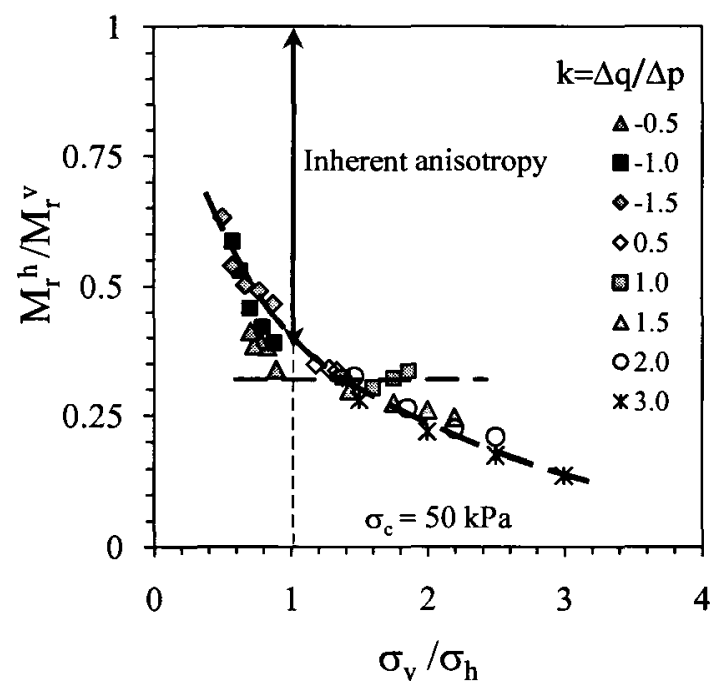

(a)

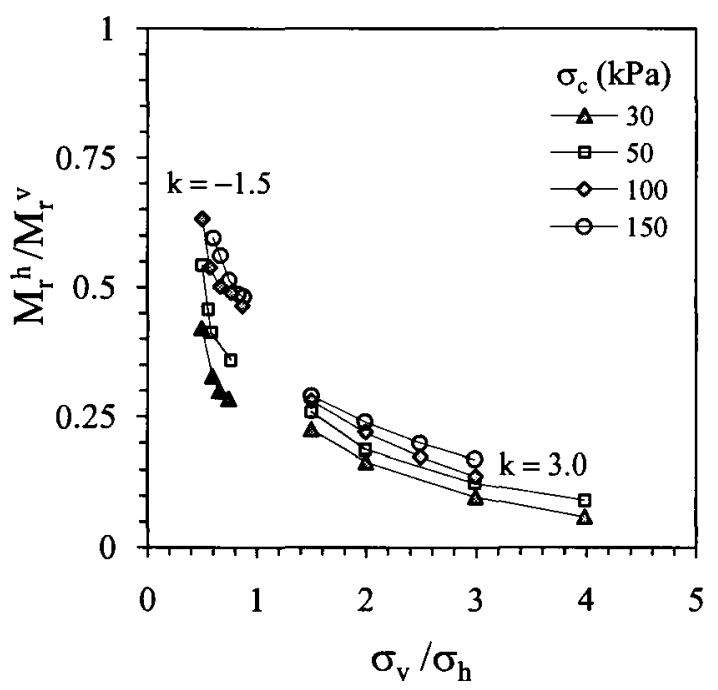

(b)

Fig. 4.26 Stress-induced stiffness anisotropy

Jiang et.al. (1997) and Tasuoka (2000) proposed that $E_{h}^{o} / E_{v}^{o}$ at very small strain decreases in proportion to $\sigma_{h} / \sigma_{v}$. Referring to Eqs. (2.5) and (2.6), $E_{h}^{o} / E_{v}^{o}$ can be expressed as

$$
\frac{E_{h}^{o}}{E_{v}^{o}}=\frac{E_{h_{r}}^{o}}{E_{v_{r}}^{o}}\left(\frac{\sigma_{h}}{\sigma_{v}}\right)^{m}
$$

Equation (4.28) implies that the stiffness anisotropy is independent of initial confinement. The results from this study, however, show that $M_{r}^{h} / M_{r}^{v}$ not only depends on $\sigma_{v} / \sigma_{h}$, but also on $\sigma_{c}$. It is well recognized that for a given stress path, an increase in the initial confinement can reduce the stiffness anisotropy, as shown in Fig. 4.26(b). Figures 4.25 and 4.26 imply that the initial stress state has an important influence on the fabric anisotropy and stiffness anisotropy. The stress-induced stiffness anisotropy can be approximated as a function of $\sigma_{c}$ and $\sigma_{v} / \sigma_{h}$ in the following form 


$$
\frac{M_{r}^{h}}{M_{r}^{v}}=m_{0}\left(\frac{\sigma_{c}}{p_{r}}\right)^{m_{c}}\left(\frac{\sigma_{v}}{\sigma_{h}}\right)^{m_{1}}
$$

where $m_{0}>0, m_{1}<0$ and $m_{c}>0$ are regression constants, $p_{r}=1 \mathrm{kPa}$.

Equation (4.29) was used to fit the calculated ratios of $M_{r}^{h} / M_{r}^{v}$ from stress path tests with $k \neq 0$. Figure 4.27 compares the predicted $M_{r}^{h} / M_{r}^{v}$ and calculated $M_{r}^{h} / M_{r}^{v}$. The regression coefficients and $R^{2}$ are also listed. The equation is seen to provide a good fit to the calculated $M_{r}^{h} / M_{r}^{v}$ for stress path tests with $k \neq 0$.

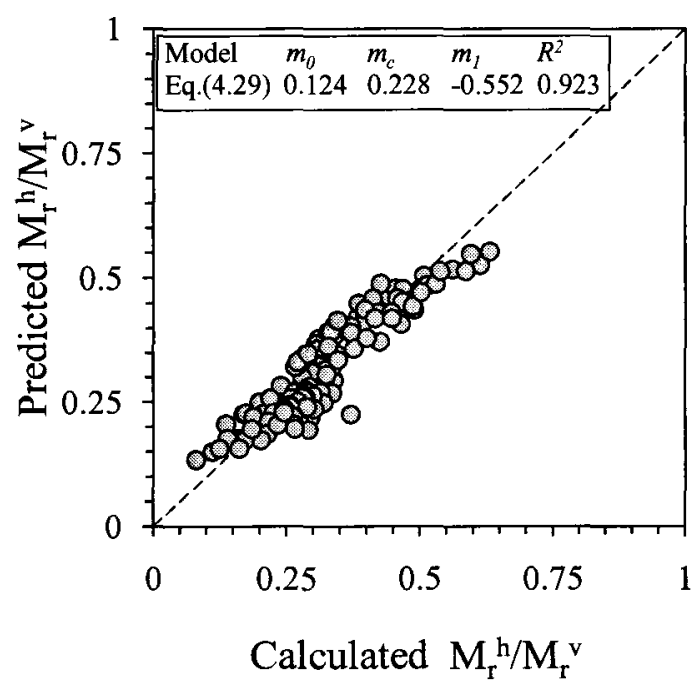

Fig. 4.27 Predicted and calculated modulus ratio for stress paths with $k \neq 0$

\section{(b) Inherent anisotropy}

After compaction during construction or sample fabrication stage, the granular material develops an anisotropic fabric, which leads to an inherent anisotropy even when the material is subjected to hydrostatic stresses.

Figure 4.28 shows that the inherent stiffness anisotropy obtained from the results of 
IC tests. It can be seen that $M_{r}^{h} / M_{r}^{v}$ increases with $\sigma_{c}$, indicating that $M_{r}^{h}$ gradually approaches $M_{r}^{v}$ and the material becomes less anisotropic. When $\sigma_{c}$ exceeds a certain level, the material may become isotropic. The subsequent stress changes however have little influence on the stiffness anisotropy, at least for the conditions investigated in this research. Therefore, the resilient modulus ratio $M_{r}^{h} / M_{r}^{v}$ used to quantify the inherent stiffness anisotropy for an isotropic initial stress state can be expressed as a power function of $\sigma_{c}$.

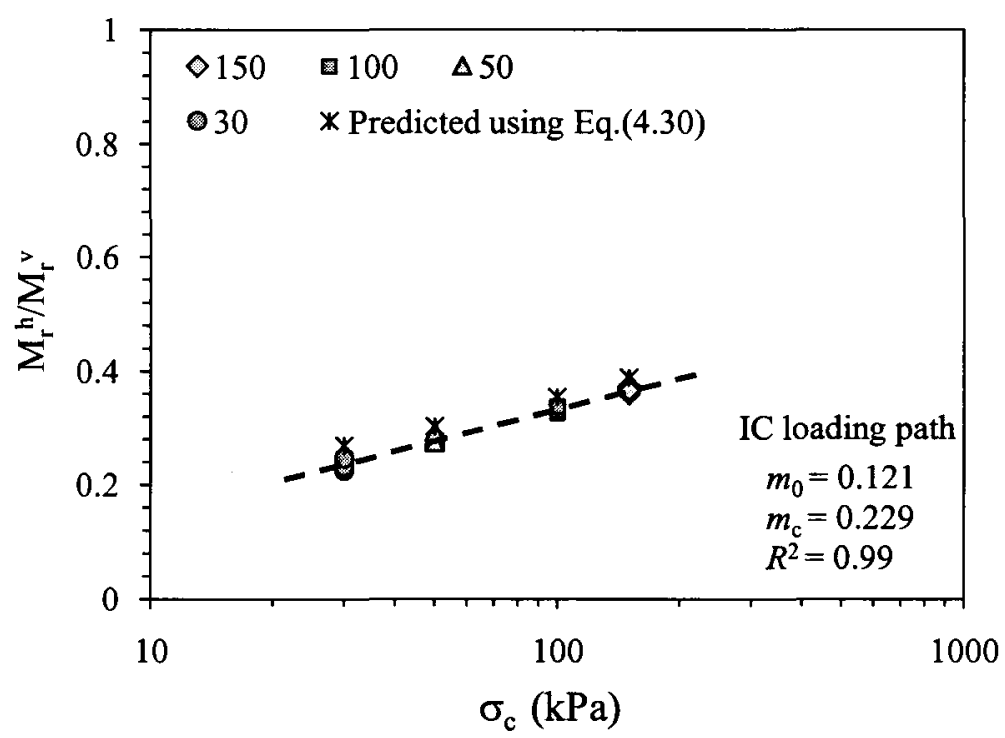

Fig. 4.28 Inherent stiffness anisotropy

An alternative way to identify the inherent stiffness anisotropy is to predict it from Eq. (4.29), which is used to provide an estimate of the stress-induced stiffness anisotropy in previous section. When $\sigma_{v} / \sigma_{h}=1$, the term $\left(\sigma_{v} / \sigma_{h}\right)^{m_{1}}$ in Eq. (4.29) can be removed. Thus Eq. (4.29) becomes 


$$
\left(\frac{M_{r}^{h}}{M_{r}^{v}}\right)_{\text {inherent }}=m_{0}\left(\frac{\sigma_{c}}{p_{r}}\right)^{m_{c}}
$$

Equation (4.30) indicates that $M_{r}^{h} / M_{r}^{v}$ which is used to quantify the inherent stiffness anisotropy for an isotropic initial stress state depends on $\sigma_{c}$ and $m_{0}$. It can be seen from Fig. 4.30 that the inherent stiffness anisotropy predicted using Eq. (4.30) is very close to that obtained from the results of IC tests.

\subsection{RESILIENT MODULUS EQUATION}

The resilient modulus test results for different stress paths reveal that the Uzan and AASHTO resilient modulus equations, which are currently used in engineering practice, are not able to properly characterize the resilient behaviour of granular materials for general stress conditions.

In pavement engineering, the preferred method of modelling $M_{r}$ is to estimate $M_{r}$ based on stress variables such as mean effective stress, bulk stress, deviator stress. As discussed already, the tests results have shown that the influence of stresses on $M_{r}$ is stress path dependent. Therefore, it is not a trivial task to estimate a design $M_{r}$ under general stress states solely using stress variables. In soil mechanics, the strain-level dependency of modulus has been well recognized for a long time. As such, an attempt is made in this section to relate $M_{r}$ with both stress and strain quantities. Considering the various deformation characteristics and variation of resilient moduli for different stress paths, it is unlikely that one can describe the complex resilient behaviour with a single formulation. Different formulations are proposed for different stages of a stress path. 


\subsubsection{Stress path with $k>0$}

The significant influence of confining pressure on resilient modulus is well established; i.e., both $M_{r}^{h}$ and $M_{r}^{v}$ increase with an increase in the confining pressure. In addition, the test results have shown that both $M_{r}^{h}$ and $M_{r}^{v}$ can be expressed as functions of $\varepsilon_{h, r}$. When $\varepsilon_{h, r}>0, M_{r}^{v}$ increases as $\varepsilon_{h, r}$ increases; When $\varepsilon_{h, r}<0, M_{r}^{v}$ also increases as the amplitude of $\varepsilon_{h, r}$ increases, but in a different manner as that when $\varepsilon_{h, r}>0$. It is noteworthy that the material may experience stress/strain softening when the load level exceeds the load applied in this study. Therefore, $M_{r}^{v}$ for $k>0$ can be described as

$$
\begin{gathered}
\text { for } \varepsilon_{h, r}>0 \\
\qquad \begin{array}{c}
M_{r}^{v}=k_{1} \sigma_{c}{ }^{k_{2}}\left(\varepsilon_{h, r}^{2}+k_{3} \varepsilon_{h, r}+k_{4}\right) \\
\text { or } \\
\text { for } \varepsilon_{h, r}<0 \quad M_{r}^{v}=k_{1}^{\prime} \sigma_{c}{ }^{k_{2}^{\prime}} \varepsilon_{h, r}^{k_{3}^{\prime}}
\end{array} \\
M_{r}^{v}=k_{5} \sigma_{c}^{{ }^{k}}\left(\begin{array}{c}
\left.k_{h, r}^{2}+k_{7} \varepsilon_{h, r}+k_{8}\right) \\
\text { or } \\
M_{r}^{v}=k_{5}^{\prime} \sigma_{c}{ }^{k_{6}^{\prime}}\left|\varepsilon_{h, r}\right|^{k_{7}^{\prime}}
\end{array}\right.
\end{gathered}
$$

where $k_{1} \sim k_{8}, k_{1}^{\prime} \sim k_{3}^{\prime}$, and $k_{5}^{\prime} \sim k_{7}^{\prime}$ are regression constants. Equations (4.31) and (4.33) are able to simulate the stress/strain softening resilient behaviour of granular materials.

$M_{r}^{h}$, on the other hand, is observed to consistently increase as $\varepsilon_{h, r}$ increases, and follows an exponential law

$$
M_{r}^{h}=k_{9} \sigma_{c}^{k_{10}} \exp ^{k_{11} \varepsilon_{h, r}}
$$

where $k_{9} \sim k_{11}$ are regression constants.

As no obvious stress/strain softening was found in this study, Eqs. (4.32), (4.34) and (4.35) are used to fit the measured $M_{r}^{v}$ and $M_{r}^{h}$, respectively. As mentioned previously, the trend of $M_{r}^{v}$ for $k=2.0$ seems somehow different from the other stress paths due to 
very small $\varepsilon_{h, r}$ induced. When doing the curve fitting, the measured data for $k=2.0$ was however also included. Figure 4.29 compares the predicted resilient moduli and measured ones. The coefficients and $R^{2}$ are also listed. The figure shows that the proposed equations fit the measured resilient moduli for $k>0$ fairly well.
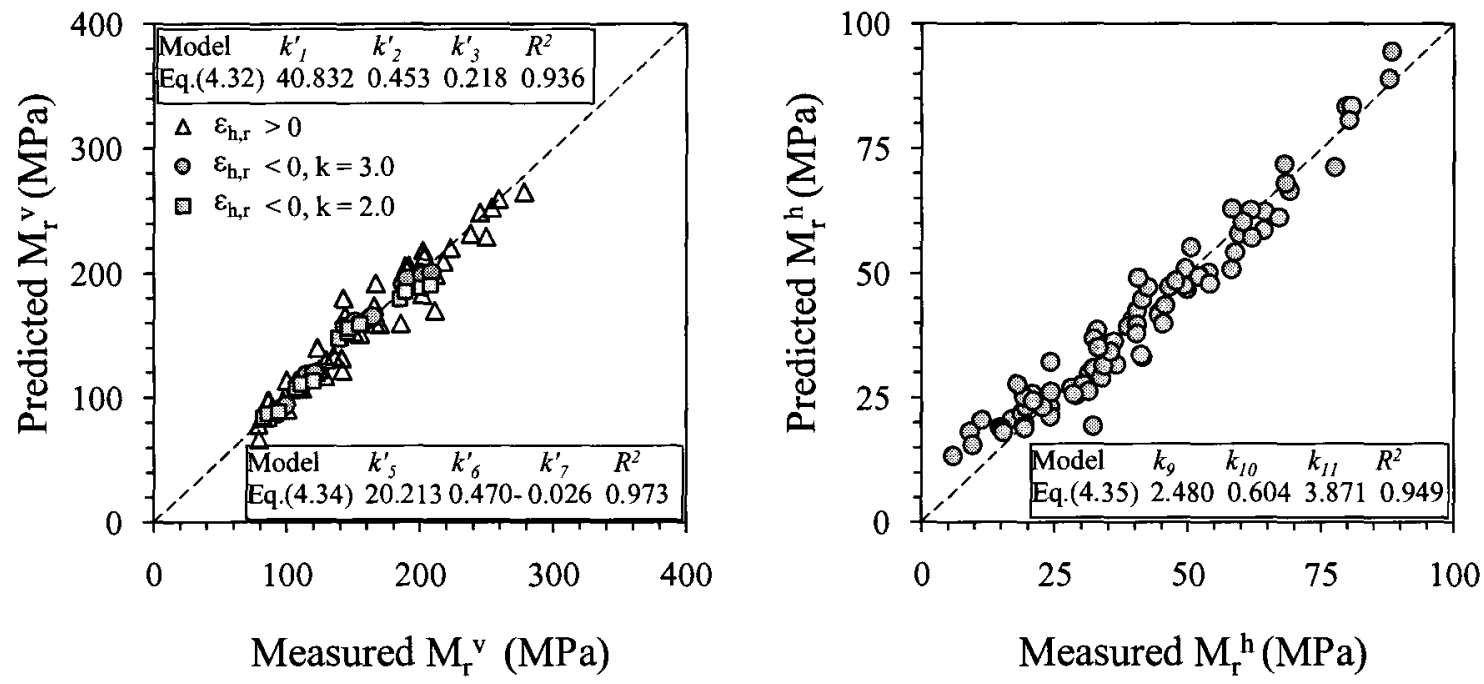

Fig. 4.29 Predicted and measured resilient moduli from positive stress paths

\subsubsection{Stress path with $k<0$}

When the major principal stress is in the horizontal direction, $M_{r}^{v}$ and $M_{r}^{h}$ are shown to depend on $\varepsilon_{v, r}$ and $\varepsilon_{h, r}$, respectively. $M_{r}^{v}$ is found to consistently increase as $\varepsilon_{v, r}$ increases, with extensive $\varepsilon_{v, r}$ yielding lower $M_{r}^{v}$, and $M_{r}^{h}$ consistently increases as $\varepsilon_{h, r}$ increases, with smaller $\varepsilon_{h, r}$ yielding lower $M_{r}^{h}$. Similar to $M_{r}^{h}$ for $k>0, M_{r}^{v}$ and $M_{r}^{h}$ for $k<0$ can be described as

$$
M_{r}^{v}=k_{12} \sigma_{c}^{k_{13}} \exp ^{k_{14} \varepsilon_{v, r}}
$$




$$
M_{r}^{h}=k_{15} \sigma_{c}^{k_{16}} \exp ^{k_{17} \varepsilon_{h, r}}
$$

where $k_{12} \sim k_{17}$ are regression constants.

Equations (4.36) and (4.37) were used to fit the measured $M_{r}^{v}$ and $M_{r}^{h}$ from stress path tests with $k<0$, respectively. Figure 4.30 compares the predicted resilient moduli and measured ones. The coefficients and $R^{2}$ are also listed. The figure shows that the proposed equations give good fit to the measured resilient moduli.
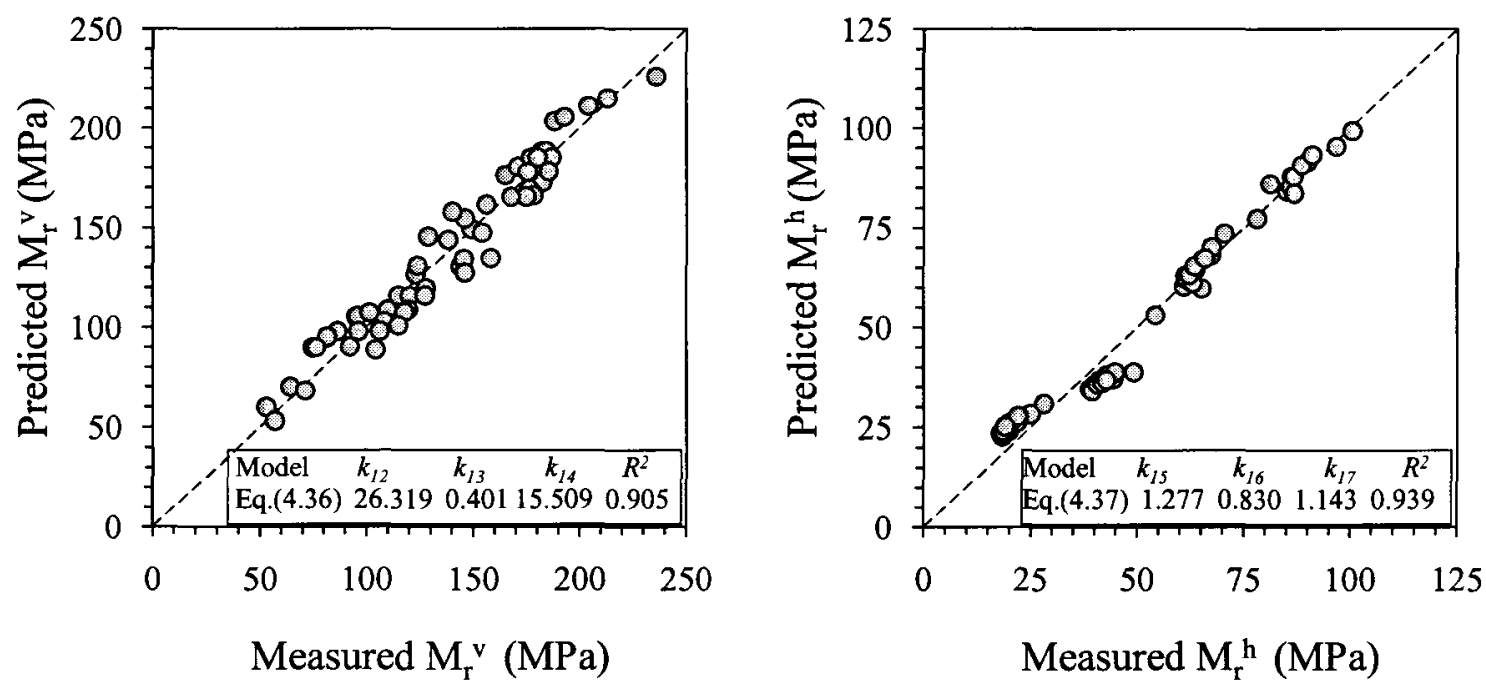

Fig. 4.30 Predicted and measured resilient moduli from negative stress paths

\subsubsection{IC loading path}

The analysis of $M_{r}^{v}$ and $M_{r}^{h}$ data obtained from IC loading paths shows that $M_{r}^{v}$ and $M_{r}^{h}$ can be estimated using $\sigma_{c}$ and $\sigma_{c}^{\prime}$ using the following relations:

$$
\begin{aligned}
& M_{r}^{v}=k_{18}{\sigma_{c}}^{k_{19}}{\sigma_{c}^{\prime}}^{k_{20}} \\
& M_{r}^{h}=k_{21}{\sigma_{c}}^{k_{22}}{\sigma_{c}^{\prime k_{23}}}^{k_{23}}
\end{aligned}
$$


where $k_{18} \sim k_{23}$ are regression constants. In these expressions, $\sigma_{c}$ reflects the influence of initial confinement on inherent fabric while $\sigma_{c}^{\prime}$ reflects the influence of stress increments.

Alternatively, $M_{r}^{v}$ and $M_{r}^{h}$ from an IC loading path can be estimated by $\sigma_{c}$ and the induced strain $\varepsilon_{v, r}$ or $\varepsilon_{h, r}$, since $\varepsilon_{h, r} / \varepsilon_{v, r}$ can be considered as constant along the loading path for a given $\sigma_{c}$.

$$
\begin{aligned}
& M_{r}^{v}=k_{24} \sigma_{c}{ }^{k_{25}} \varepsilon_{v, r}{ }^{k_{26}} \\
& M_{r}^{h}=k_{27} \sigma_{c}{ }^{k_{28}} \varepsilon_{v, r}{ }^{k_{29}}
\end{aligned}
$$

where $k_{24} \sim k_{29}$ are regression constants.

Equations (4.38) through (4.41) were used to fit the measured $M_{r}^{v}$ and $M_{r}^{h}$ from IC tests, respectively. Figure 4.31 compares the predicted resilient moduli and measured ones. It shows that the equations are capable of estimating $M_{r}^{v}$ and $M_{r}^{h}$ with confidence for the IC loading paths.
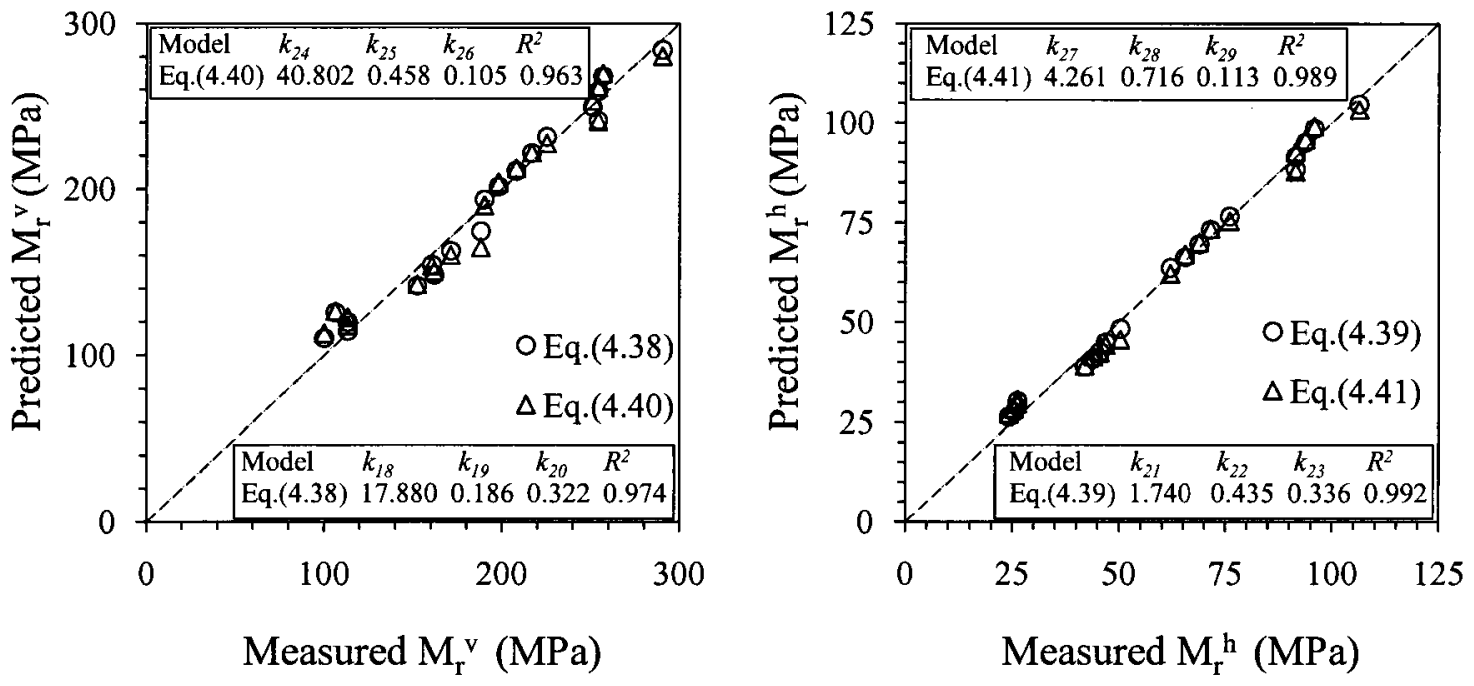

Fig. 4.31 Predicted and measured resilient moduli from IC loading paths 
One observes that the coefficients $k_{20}$ and $k_{23}$ are very close, indicating that $\sigma_{c}^{\prime}$ has little influence on $M_{r}^{h} / M_{r}^{v}$. The result is consistent with that in Eq. (4.30). In contrast, the initial confining pressure $\sigma_{c}$ has more significant influence on the stiffness anisotropy, which can be seen from the coefficients obtained from the regression analysis.

\subsection{SELECTION OF RESILIENT POISSON'S RATIO}

The variations in $v_{v h}$ and $v_{h h}$ with stresses were presented earlier in Fig. 4.24. Similar to resilient modulus, it is unlikely that one can use a single formulation to model the resilient Poisson's ratios. Based on the observation and analysis of the previous sections, the approximations for both $v_{v h}$ and $v_{h h}$ depend on stress paths, or the deformation characteristics.

For the IC loading paths and stress paths with $k>0$, when the material is contractive in both directions (e.g. $k=0 \sim 1.5$ ), the variations of $v_{v h}$ and $v_{h h}$ with $\sigma_{v} / \sigma_{h}$ are rather small. For simplicity and easy implementation in engineering practice, both $v_{v h}$ and $v_{h h}$ may be considered as constant. The variations of $v_{v h}$ and $v_{h h}$ for stress paths with $k<0$ are small and can also be considered approximately as constant.

For stress paths with noticeable extensive $\varepsilon_{h, r}\left(\right.$ e.g. $k=2.0$ and 3.0), $v_{v h}$ and $v_{h h}$ increase as $\sigma_{v} / \sigma_{h}$ increases. The ranges of $v_{v h}$ and $v_{h h}$ are much wider than those of other stress paths without extensive horizontal strains, which have a remarkable influence on resilient dilatancy. In the structural analysis of flexible pavement, one of the critical locations is the bottom of base right beneath the wheel load, where extensive radial strain is usually observed. Therefore, $v_{v h}$ and $v_{h h}$ at the bottom of base layer right beneath the 
wheel load or for stress paths with $k=2.0$ and 3.0 cannot be taken as constant for realistic analysis. Equation (2.32), a third degree polynomial proposed by Hicks and Monismith (1971) or truncated second degree polynomial, can be used to predict the resilient Poisson's ratios, with different coefficients for $v_{v h}$ and $v_{h h}$ :

$$
\begin{aligned}
& v_{v h}=a_{0}+a_{1}\left(\frac{\sigma_{v}}{\sigma_{h}}\right)+a_{2}\left(\frac{\sigma_{v}}{\sigma_{h}}\right)^{2} \\
& v_{h h}=b_{0}+b_{1}\left(\frac{\sigma_{v}}{\sigma_{h}}\right)+b_{2}\left(\frac{\sigma_{v}}{\sigma_{h}}\right)^{2}
\end{aligned}
$$

where the coefficients $a_{0} \sim a_{2}$ and $b_{0} \sim b_{2}$ are functions of $k$, as shown in Fig. (4.24)

Equations (4.42) and (4.43) were then used to fit the measured $v_{v h}$ and $v_{h h}$ from stress path tests with $k=2.0$ and 3.0 , respectively. Figure 4.32 compares the predicted resilient Poisson's ratios and measured ones. It shows that the proposed resilient Poisson's ratio equations are "good enough" to estimate the Poisson's ratios along stress paths where extensive lateral strains are induced.
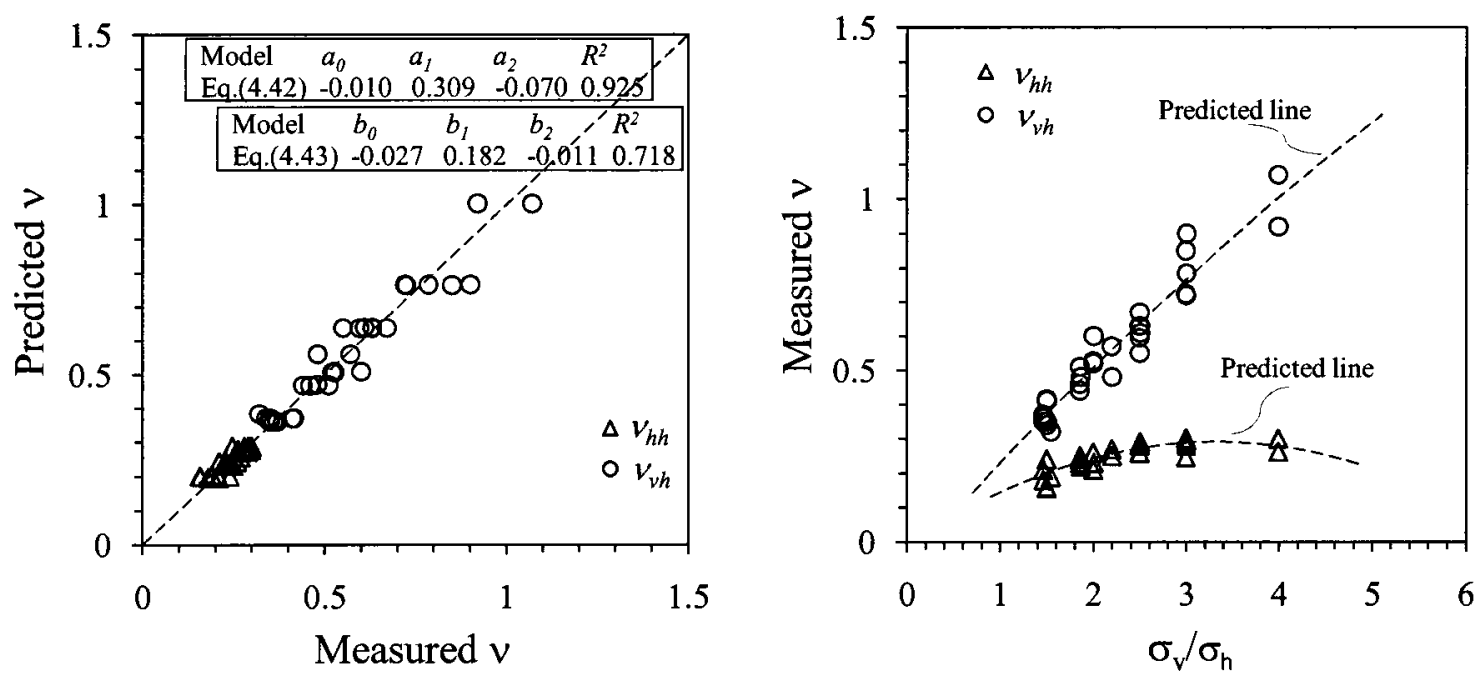

Fig. 4.32 Predicted and measured Poisson's ratio for $k=2.0$ and $k=3.0$ 


\subsection{SUMMARY}

This chapter attempted to explore the cross-anisotropic resilient behaviour of initially isotropic consolidated granular materials along different stress paths. Different deformation characteristics were observed for the stress path tests performed in the study. In general, the possible combinations of deformations are: contractive in both the vertical and horizontal directions; contractive in the vertical/horizontal direction and extensive in the horizontal/vertical direction; and contractive in the vertical/horizontal direction and very small strain in the horizontal/vertical direction. The different patterns of stress-strain curves are attributed to both the anisotropic nature of the material and the influence of applied stresses. Owing to the various deformation characteristics, the variation of resilient modulus with stresses depends on stress path. For all stress path tests performed, $M_{r}^{h}$ is consistently lower than $M_{r}^{v}$. For stress paths with positive $k, M_{r}^{v}$ increases with the stress, but $M_{r}^{h}$ may decrease with the stress when $k$ increases, as a result of the onset of extensive horizontal resilient strain. For stress paths with negative $k, M_{r}^{h}$ increases as the stress increases, but $M_{r}^{v}$ may decrease when extensive vertical resilient strain develops.

The inherent stiffness anisotropy of the test material is found to depend on initial confining pressure. When $\sigma_{c}$ increases, the stiffness anisotropy decrease, indicating the material becomes less anisotropic. It is found that for a given $\sigma_{c}$, the stiffness anisotropy from the IC tests almost remains constant, independent of the subsequent stress changes. For stress paths with $k>0$, the stress-induced stiffness anisotropy increases as the stresses become more anisotropic; i.e., $\sigma_{v} / \sigma_{h}$ increases. However, when the major principal stresses are in horizontal directions, the stress-induced stiffness anisotropy 
decreases as $\sigma_{v} / \sigma_{h}$ decreases. In general, the stress-induced anisotropy depends on $\sigma_{c}$ and $\sigma_{v} / \sigma_{h}$, with $M_{r}^{h} / M_{r}^{v}$ decreasing and increasing as $\sigma_{c}$ and $\sigma_{v} / \sigma_{h}$ increase, respectively.

Test results show that the common preferred resilient modulus equations, such as the Uzan and AASHTO resilient modulus equations, are not capable of characterizing the cross-anisotropic resilient modulus for general stress paths. Based on the observation and analysis, several equations are proposed to approximate the cross-anisotropic resilient properties using combination of stress variables or stress and strain variables for different stress paths. It is found that for stress paths with $k \neq 0, M_{r}^{v}$ can be expressed as a function of $\sigma_{c}$ and the resilient strain in the direction of minor principal stress. And $M_{r}^{h}$ can be approximated as a function of $\sigma_{c}$ and $\varepsilon_{h, r}$. It has been shown that the proposed equations give good fit to the measured data in the stress path tests. 


\section{RESILIENT BEHAVIOUR FOR ANISOTROPIC CONSOLIDATION}

\subsection{INTRODUCTION}

This chapter presents the resilient modulus test results under conditions of anisotropic consolidation. In particular, the resilient responses, and the stress/strain dependency of resilient moduli and Poisson's ratios corresponding to various anisotropic consolidation histories, are described. As explained in Section 4.3, the moduli and Poisson's ratios were obtained by carrying out stress path tests at the various stress levels and applying the least square fit together with the Graham-Houlsby assumption. Based on the test results, the effect of $K_{\text {ini }}$ on the resilient properties of granular materials for various initial stress states is identified. The influence of $K_{i n i}$ on the stiffness anisotropy is also investigated. Finally, the resilient modulus equations developed in Chapter 4 are extended to take into account of the effect of $K_{i n i}$ and stress/strain path on resilient properties of granular materials for general axi-symmetric stress conditions that are commonly experienced by base layers.

\subsection{RESILIENT STRESSES AND STRAINS}

For standard $M_{r}$ tests, a seating load corresponding to a small deviator stress in axial direction is applied to maintain a good contact between the top loading plate and the specimen. Since the deviator stress induced by the seating load is relatively small compared to the applied confining pressure, the initial stress states can be considered as 
approximately isotropic. However, as mentioned previously, based on in-situ measurements, residual horizontal stresses may be larger than the overburden stress, implying that $K_{\text {ini }}$ in a granular base layer can be greater than unity. Nevertheless, the commonly used resilient modulus equations, such as the Uzan and AASHTO resilient modulus equations, which originated from standard $M_{r}$ tests, assumes that $M_{r}$ depends only on the final stress states (i.e., bulk stress and deviator stress corresponding to the peak stresses during cyclic loading), with the effect of initial stress state and $K_{\text {ini }}$ being neglected. In this study, the effect of initial stress state and $K_{i n i}$ is investigated by performing $M_{r}$ tests on compacted specimens subjected to consolidation with different $K_{\text {ini }}$ values. In each test, the stresses prior to applying cyclic stresses (i.e., at the end of a consolidation stage) are taken as the initial stresses for $M_{r}$ tests.

Figure 5.1 shows the variation in void ratio before and after consolidation for stress paths corresponding to $k=3.0$ and -1.5 , respectively. It can be clearly seen that initial stresses have a negligible influence on void ratios of the densely compacted specimens.
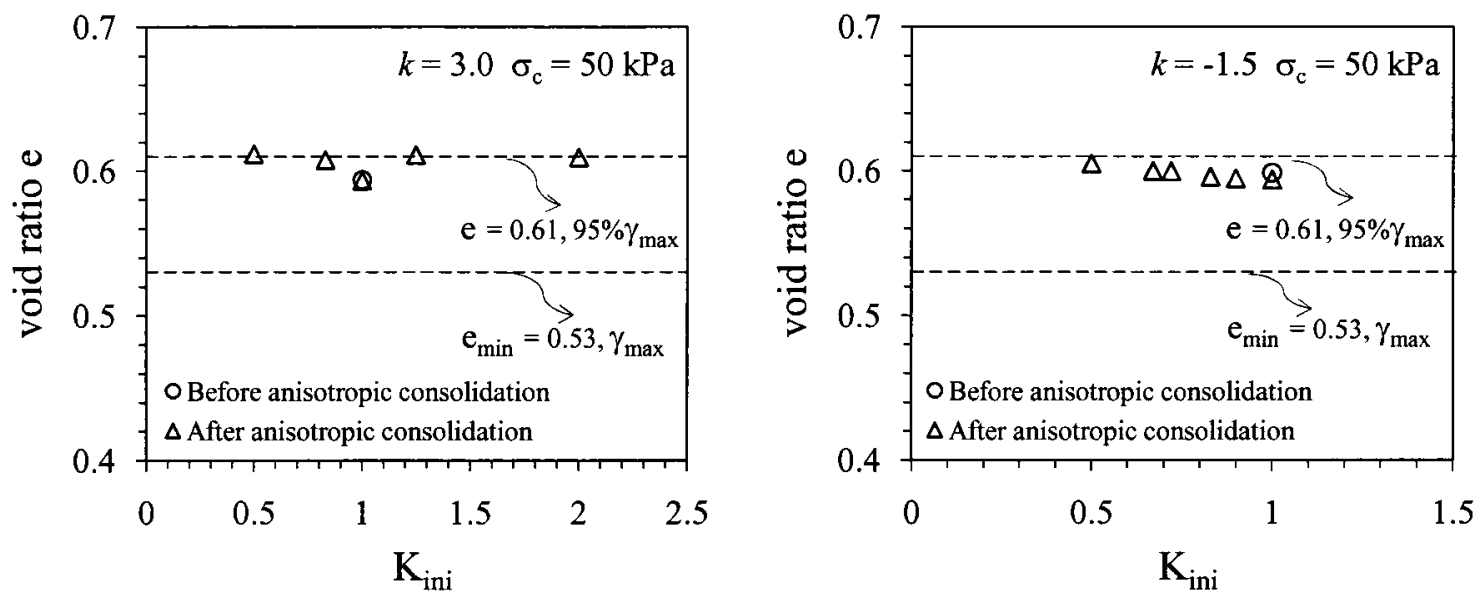

Fig. 5.1 Variation of void ratios before and after anisotropic consolidation 
Initial stress states may however play an important role on the resilient responses of granular materials. Figure 5.2 presents the relations between the repeated vertical stresses and resilient strains for various $K_{\text {ini }}$ when $k=3.0$ and $\sigma_{c}=50 \mathrm{kPa}$. It is observed that the resilient responses vary with the initial stress states, even though the finial stress states are the same. The resistance of deformation to the vertical stress increases as $K_{i n i}$ decreases. Whereas $\sigma_{d}$ for $K_{\text {ini }}=2$ is 2.5 times that for $K_{\text {ini }}=0.5$ when $\sigma_{v}=150 \mathrm{kPa}$, $\varepsilon_{v, r}$ increases by approximately a factor 6 and the magnitude of extensive $\varepsilon_{h, r}$ increases by approximately a factor 9 . Hence the resilient dilatancy is more pronounced for a higher $K_{i n i}$. The apparent Poisson's ratio $v_{v h}$ also appears to increase as $K_{i n i}$ increases.

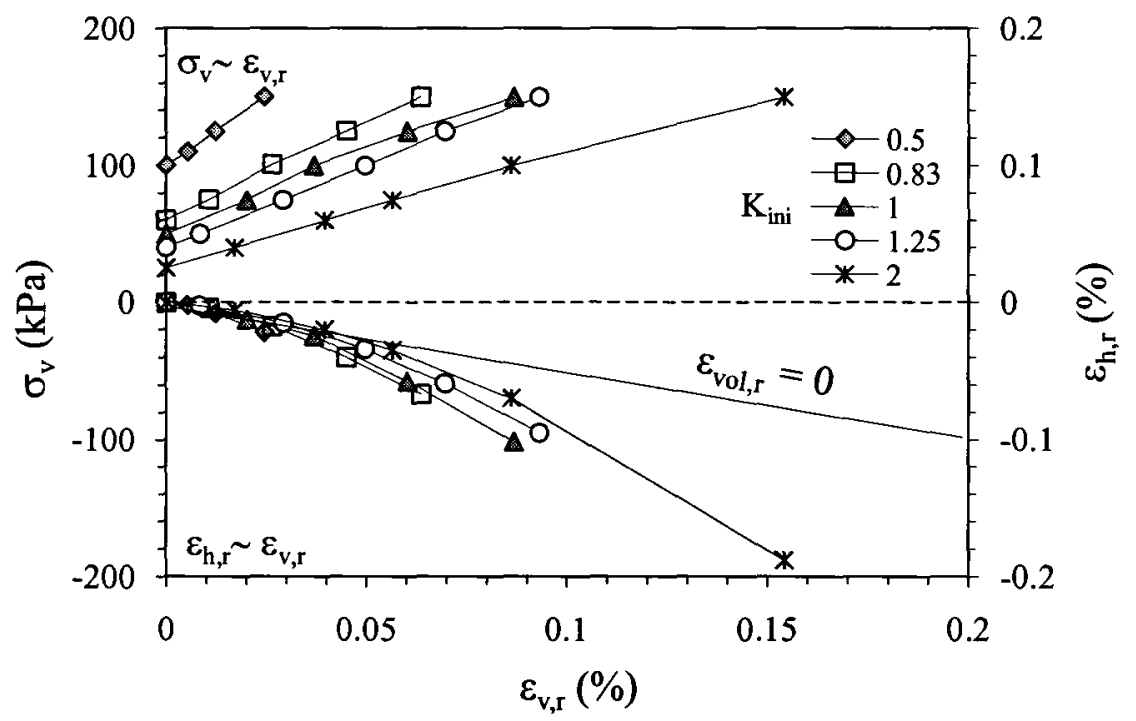

Fig. 5.2 Resilient stresses and resilient strains for $k=3.0$

$$
\sigma_{h}=50 \mathrm{kPa}
$$

When the repeated load is in the horizontal direction, the differences in the resilient deformation response for various $K_{i n i}$ conditions become smaller. As shown in Fig. 5.3, the increase in $\varepsilon_{h, r}$ with an increases in $\sigma_{h}$, is similar for all $K_{i n i}$ although $\sigma_{v}$ is 
different. The relations of $\varepsilon_{v, r} v S \varepsilon_{h, r}$ and $\varepsilon_{v o l, r} v S \varepsilon_{h, r}$ also appear to be independent of $K_{\text {ini }}$. Similar to standard $M_{r}$ tests, the resistance of deformation to the repeated $\sigma_{h}$ for $k=-1.5$ also slightly increases as $K_{i n i}$ decreases. These observations imply that $K_{i n i}$ may have different influences on the development of resilient deformations for different directions of major principal stresses. As a result, the impact of $K_{i n i}$ on the resilient properties for different directions of major principal stresses is not same.

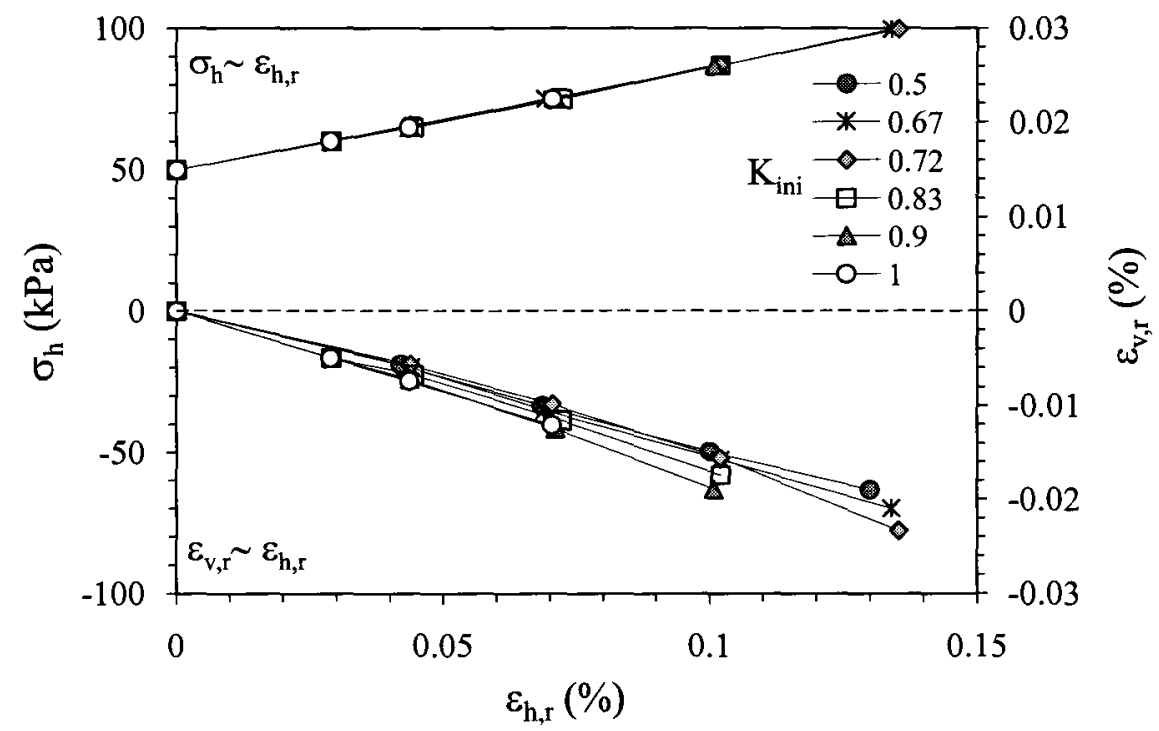

Fig. 5.3 Resilient stresses and resilient strains for $k=-1.5$

$$
\sigma_{h}^{i}=50 \mathrm{kPa}
$$

Therefore, one concludes that the resilient responses of granular material are affected by initial stress states. In other words, the resilient response for initial anisotropic stress states is different from that corresponding to isotropic stress states, which suggests that equations that neglect the influence of the initial stress state, in particular the anisotropic stress state, may not be able to characterize the resilient properties of granular materials under general stress conditions properly and need to be revised. 


\subsection{RESILIENT MODULUS}

\subsubsection{Resilient modulus and it's dependency on stress}

As the density of the specimen after consolidation in test Series B is slightly smaller than that in Series A, the trends for the variation of $M_{r}^{v}$ and $M_{r}^{h}$ in test Series B with stress are different from those of test Series A. Density-dependency of $M_{r}$ is not addressed further here, although it is recognized that $M_{r}$ does depend on density.

Figure 5.4 shows the variations of $M_{r}^{v}$ and $M_{r}^{h}$ with the applied vertical stresses (in terms of final stress states: $\mathrm{S} 1, \mathrm{~S} 2, \cdots \mathrm{S} 8$ ) for different $K_{i n i}$ conditions when $k=3.0$. As expected, the dependency of $M_{r}$ on stress is influenced by $K_{\text {ini }}$. The trends of either $M_{r}^{v}$ or $M_{r}^{h}$, as a function of stresses are similar, but the magnitudes vary with $K_{i n i}$ when the final stress states are the same. Both $M_{r}^{v}$ and $M_{r}^{h}$ tend to increase as $K_{i n i}$ decreases. $M_{r}^{v}$ for $K_{i n i}=0.5$ is found to exceed $60 \%$ of $M_{r}^{v}$ for $K_{i n i}=1$ and $M_{r}^{v}$ for $K_{i n i}=2$ is $30 \%$ lower than that for $K_{i n i}=1$. A noticeable variation in $M_{r}^{h}$ with $K_{i n i}$ is also observed.
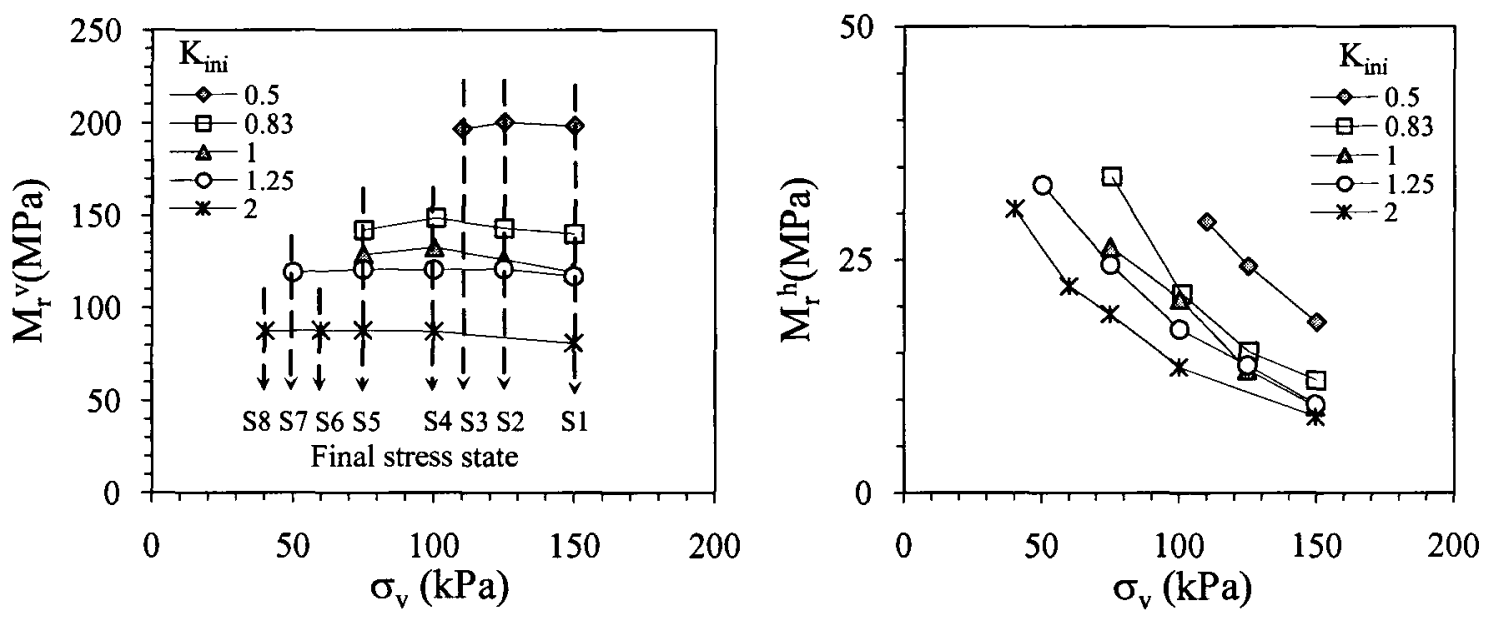

Fig. 5.4 Resilient stresses and resilient moduli for $k=3.0$ $\sigma_{h}=50 \mathrm{kPa}$ 
Figure 5.5 shows the variations of both $M_{r}^{h}$ and $M_{r}^{v}$ with $K_{i n i}$ in terms of $p$ and $\sigma_{d}$ for $k=3.0$. One observes that whereas $M_{r}^{v}$ is insensitive to $p$ and $\sigma_{d}, M_{r}^{h}$ shows sensitivity to both. And higher $M_{r}^{h}$ and $M_{r}^{v}$ are obtained for a lower $K_{i n i}$ when $p$ and $\sigma_{d}$ are fixed. As such, resilient modulus equations based on standard $M_{r}$ test results solely for isotropic consolidation stresses are not able to predict $M_{r}$ correctly.
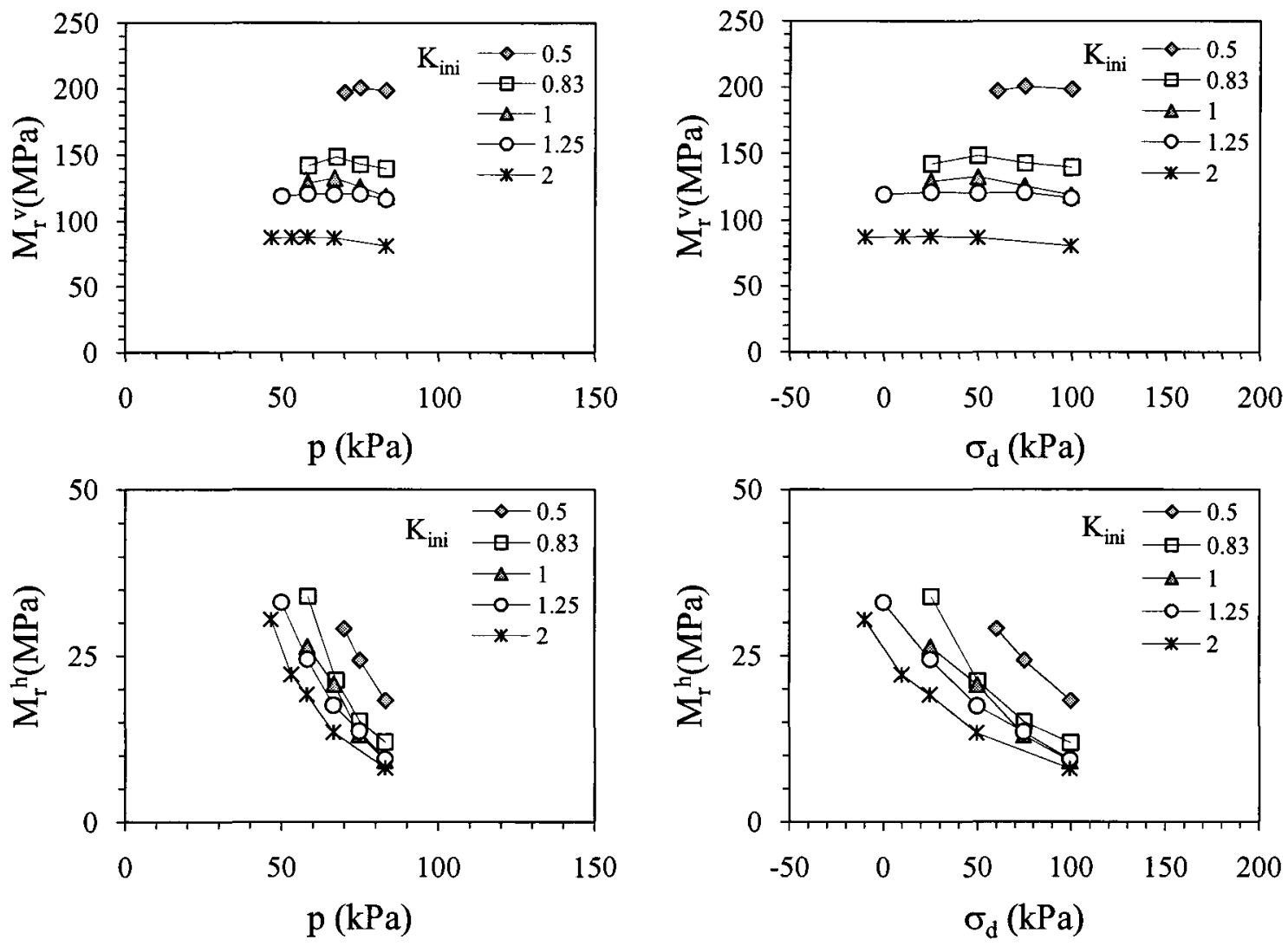

Fig. 5.5 Resilient stresses and resilient moduli for $k=3.0$

$$
\sigma_{h}=50 \mathrm{kPa}
$$

When the repeated vertical stress increment $\Delta \sigma_{v}$ is used to investigate the impact of $K_{\text {ini }}$ on resilient moduli, as shown in Fig. 5.6, one observes that $M_{r}^{v}$ is influenced significantly by $K_{i n i}$, but $\Delta \sigma_{v}$ only has minor effect on $M_{r}^{v}$. While $M_{r}^{h}$ is relatively 
insensitive to $K_{\text {ini }}$, but decreases as $\Delta \sigma_{v}$ increases.

Since $M_{r}^{v}$ for $k=3.0$ is insensitive to $\Delta \sigma_{v}$ or $\sigma_{v}$ in this study, it can be seen from Fig. 5.7 that $M_{r}^{v}$ decreases with an increase in $K_{i n i}$, independent of the final stress state, which follows a power law. It is interesting to note that $M_{r}^{v}$ has a unique relation with $K_{\text {ini }}$ at a given initial confinement $\left(\sigma_{h}^{i}=\sigma_{h}\right.$ for $\left.k=3.0\right)$, which implies that $M_{r}^{v}$ is uniquely determined by the bulk stress prior to cyclic loading since $\theta_{\text {ini }}=\left(2+\frac{1}{K_{\text {ini }}}\right) \sigma_{h}^{i}$.
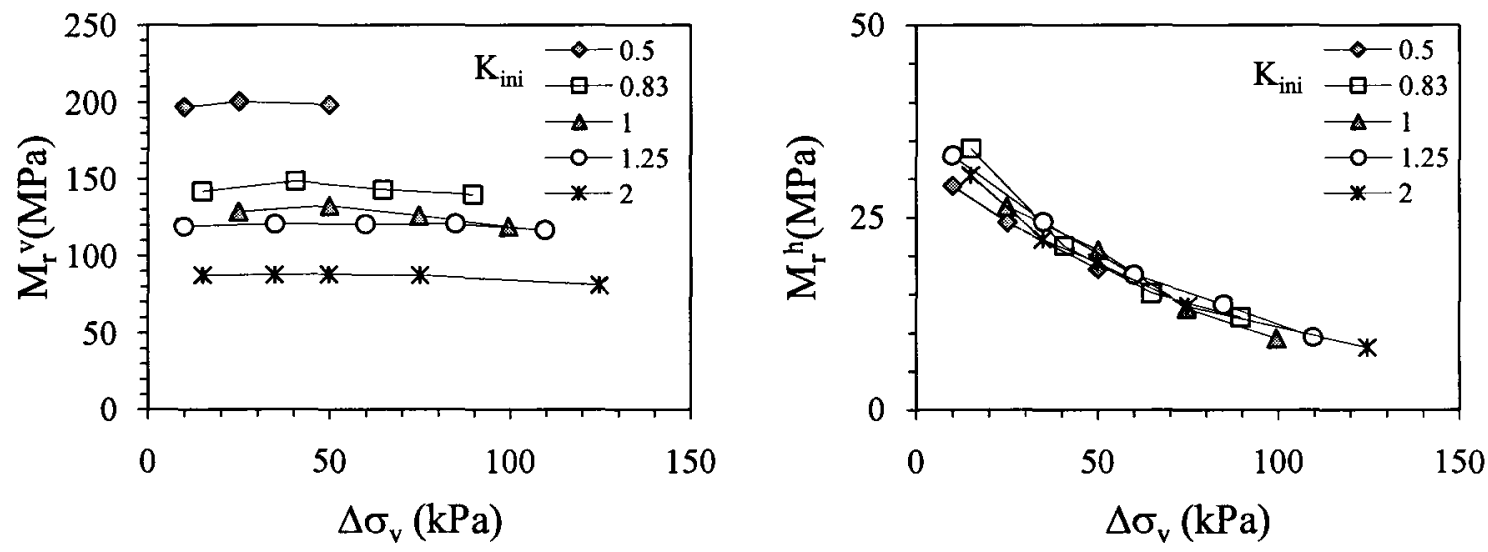

Fig. 5.6 Resilient stress increments and resilient moduli for $k=3.0$

$$
\sigma_{h}=50 \mathrm{kPa}
$$

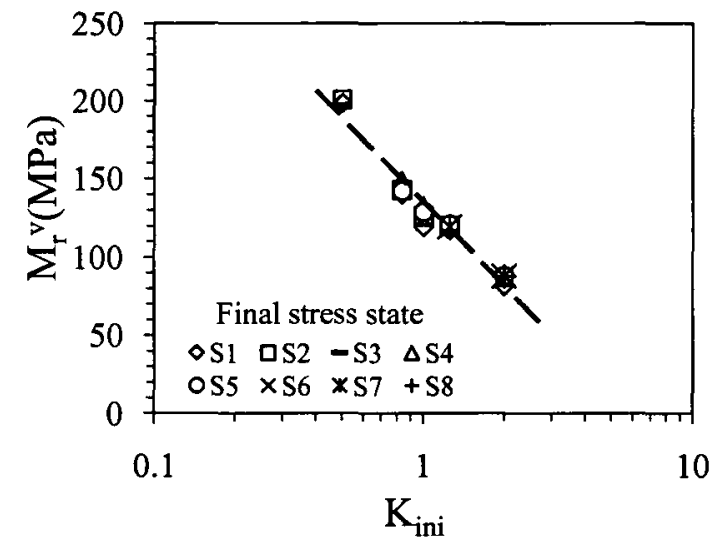

Fig. 5.7 Variation of vertical resilient modulus with $K_{\text {ini }}$

$$
k=3.0, \sigma_{h}=50 \mathrm{kPa}
$$


The resilient moduli for stress paths with $k=-1.5$ for different $K_{i n i}$ consolidation histories are presented in Fig. 5.8. As expected, the dependency of modulus, either $M_{r}^{h}$ or $M_{r}^{v}$, on $K_{i n i}$ and stress is different from that for stress paths with $k=3.0$. For a given $K_{i n i}, M_{r}^{v}$ decreases as $\sigma_{h}$ increases as a result of the onset of extensive $\varepsilon_{v, r}$. When $\sigma_{h}$ is the same, $M_{r}^{v}$ is bigger for a lower $K_{\text {ini }}$ since the vertical confinement $\sigma_{v}$ is higher. However, $M_{r}^{h}$ appears to be not influenced by $K_{i n i}$ and consistently increases with $\sigma_{h}$. The variations of $M_{r}^{h} v s \Delta \sigma_{h}$ and $M_{r}^{v} v s \Delta \sigma_{h}$ are similar to those shown in Fig. 5.8 since the initial horizontal confinements are same for all stress paths having $k=-1.5$.
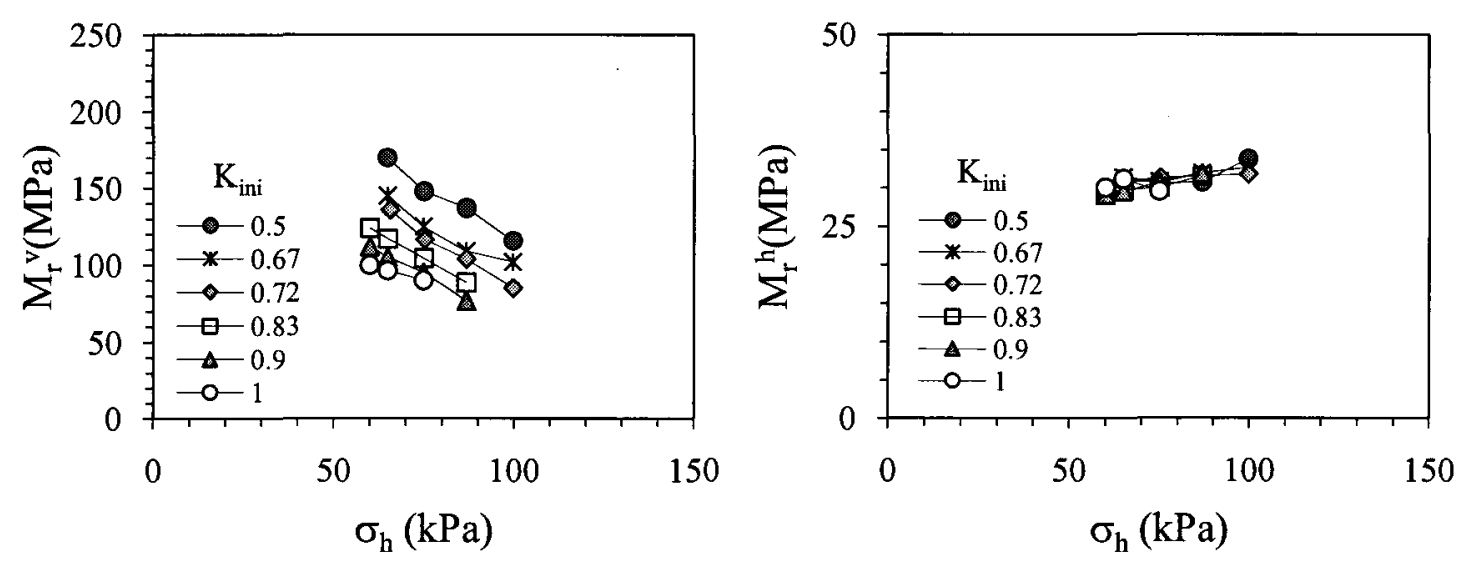

Fig. 5.8 Resilient stresses and resilient moduli for $k=-1.5$

$$
\sigma_{h}^{i}=50 \mathrm{kPa}
$$

Unlike the stress path with $k=3.0, M_{r}^{v}$ for stress path with $k=-1.5$ tends to decrease with $p$, but increases with $\sigma_{d}$; see Fig. 5.9. Given constant $p$, a higher $M_{r}^{v}$ is obtained when $K_{i n i}$ is lower. However, the dependency of $M_{r}^{v}$ on $\sigma_{d}$ is shown to be insensitive to $K_{i n i} . M_{r}^{h}$, on the other hand, increases slightly with $p$, but is almost not influenced by $\sigma_{d} . M_{r}^{h}$ is also independent of $K_{\text {ini }}$ when described in terms of $p$ and $\sigma_{d}$. 

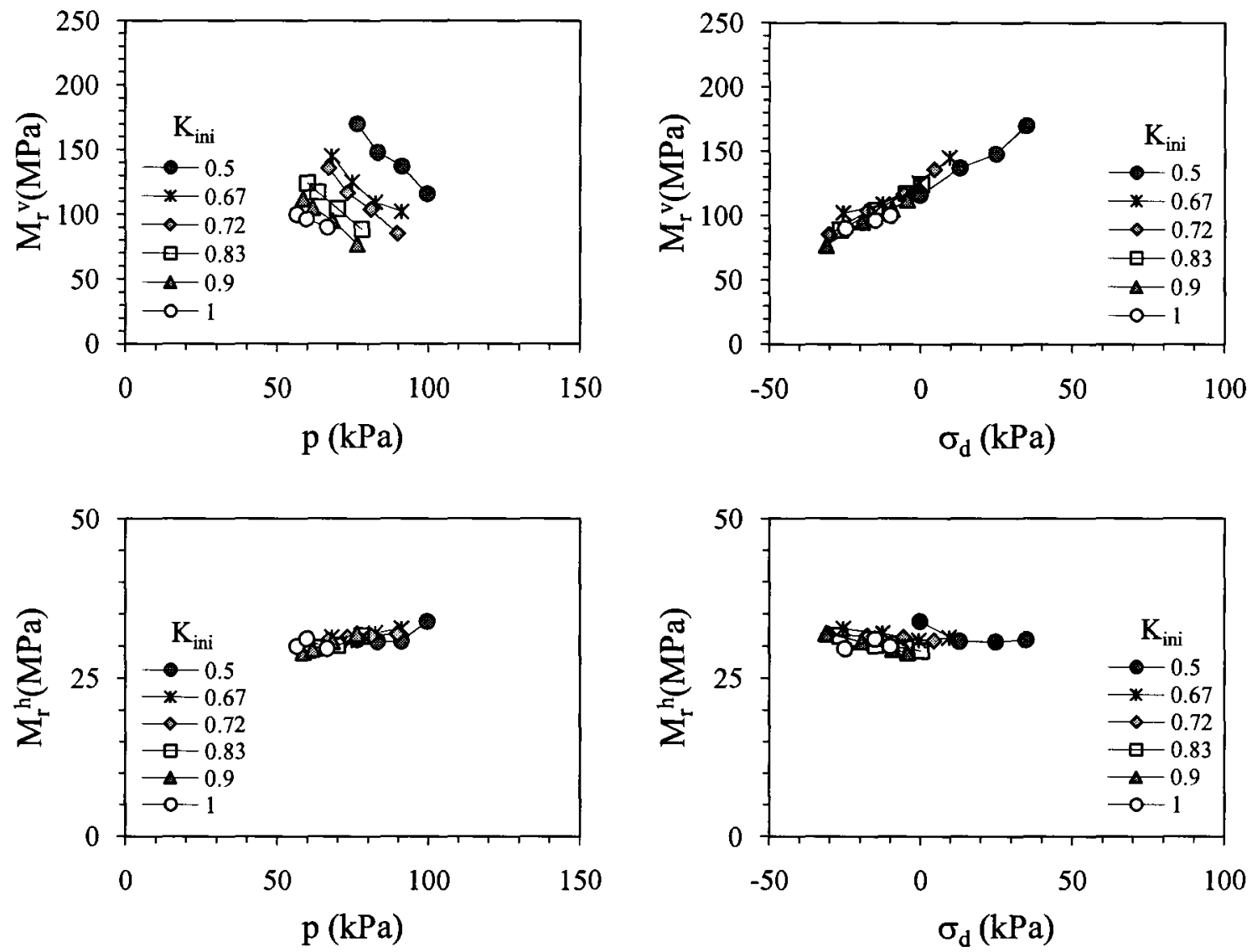

Fig. 5.9 Resilient stresses and resilient moduli for $k=-1.5$

$$
\sigma_{h}^{i}=50 \mathrm{kPa}
$$

In summary, the dependencies of $M_{r}^{h}$ and $M_{r}^{v}$ on stresses vary with the initial stress state and the stress/strain path followed. The test results imply that $M_{r}^{v}$ is more influenced by $K_{i n i}$ than $M_{r}^{h}$ for both stress paths having $k=3.0$ and -1.5 . This might be the result of soil fabric or bedding plane orientation, which need to be further investigated. In addition, the experimental results imply that if the Uzan or AASHTO resilient modulus equations are used when determining $M_{r}$ simply based on the peak stresses during cyclic loading, care must be exercised to take into account the influence of $K_{i n i}$ and stress/strain path on the resilient properties for realistic analysis. 


\subsubsection{Resilient modulus and it's dependency on resilient strain}

In Chapter 4, strain-path dependency of $M_{r}$ was recognized for samples prepared under conditions of isotropic consolidation. Figures 5.10 and 5.11 show the variation of $M_{r}^{v}$ and $M_{r}^{h}$ as a function of resilient strains for different $K_{i n i}$ and stress paths with $k=3.0$ and -1.5 , respectively. In addition to the impact of $K_{i n i}$ on $M_{r}$, it confirms the findings in the last section that $M_{r}^{v}$ is more influenced by initial stress states than $M_{r}^{h}$.
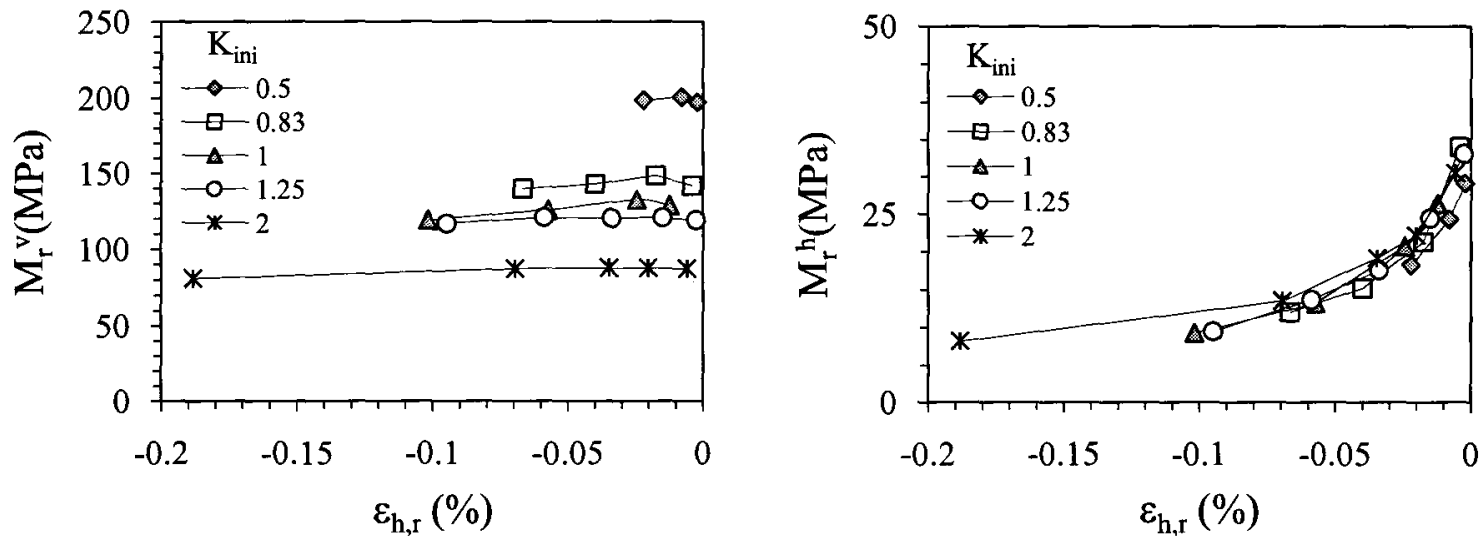

Fig. 5.10 Resilient strains and resilient moduli for $k=3.0$

$$
\sigma_{h}=50 \mathrm{kPa}
$$
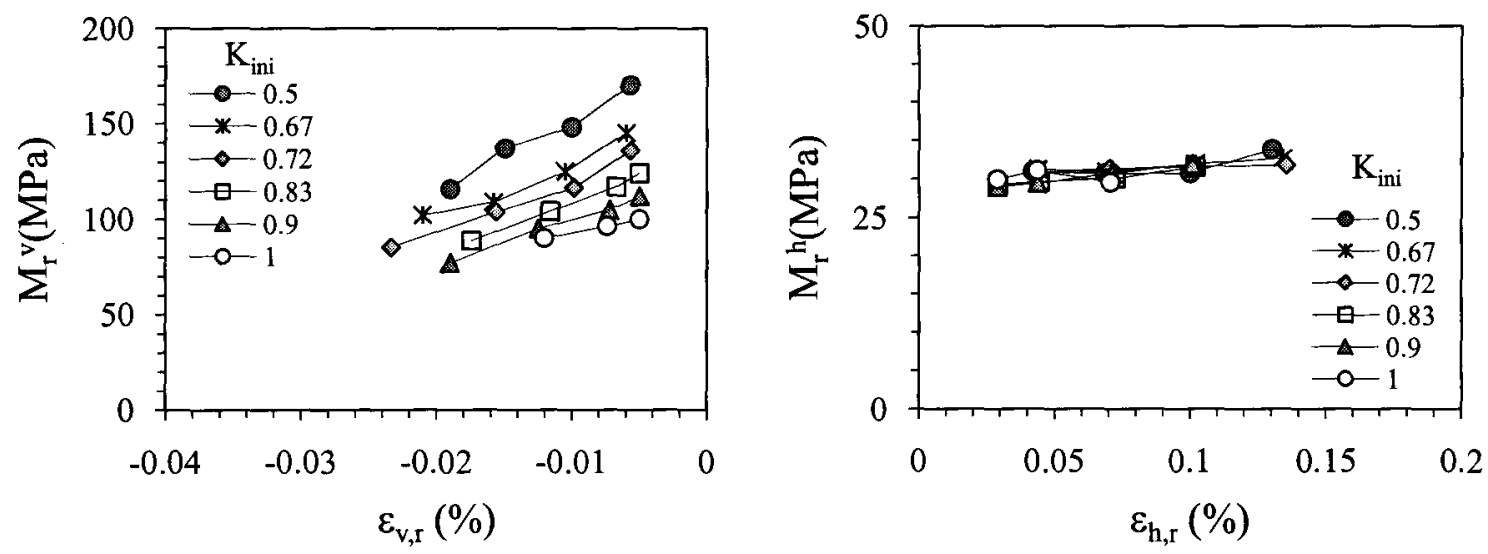

Fig. 5.11 Resilient strains and resilient moduli for $k=-1.5$

$$
\sigma_{h}^{i}=50 \mathrm{kPa}
$$


The findings about the relations of resilient moduli to resilient strains, which are generally in agreement with results presented in Figs. 4.21 and 4.22 under isotropic initial stress conditions, have important implications for pavement engineering and suggest that an alternative approach is required to characterize the resilient properties of granular materials in granular base layers.

In summary, the results from test Series A and B suggest that $M_{r}^{v}$ should be related to $K_{i n i}, \sigma_{h}^{i}$, and resilient strain in the direction of cyclic minor principal stress; i.e., $\varepsilon_{h, r}$ (for stress paths with $k>0$ ) or $\varepsilon_{v, r}$ (for stress paths with $k<0$ ), and $M_{r}^{h}$ can be related to $\sigma_{h}^{i}$ and $\varepsilon_{h, r}$. But the relations of $M_{r}^{h} v s \varepsilon_{h, r}$ for $k>0$ and $M_{r}^{h} v s \varepsilon_{h, r}$ for $k<0$ may be different as the influence of direction of major principal stress on $M_{r}^{h}$ varies.

\subsection{STIFFNESS ANISOTROPY AND STRESS}

\subsubsection{Stress-induced anisotropy}

In Chapter 4, it is recognized that under conditions of isotropic consolidation, the stressinduced stiffness anisotropy, depends on $\sigma_{v} / \sigma_{h}$ and $\sigma_{c}$. The results of test Series A clearly show that the stiffness anisotropy increases as $\sigma_{v} / \sigma_{h}$ increases; as shown in Fig. 4.26. Moreover, at a given initial confinement, the stiffness anisotropy remains constant if $\sigma_{v} / \sigma_{h}$ ratios at the final stress states (corresponding to peak stresses during cyclic loading) are identical, regardless of the history of the stress paths and the final stress states.

Figure 5.12(a) shows the dependency of stiffness anisotropy on $\sigma_{v} / \sigma_{h}$ in another way when the specimens are under various initial isotropic stress conditions. For 
example, $S_{I}^{i}$ and $S_{F}^{i}(i=1,2,3 \ldots)$ stands for initial and final stress states, respectively. When the sample is subjected to different stress path histories, such as Path $1\left(S_{I}^{2} \rightarrow S_{F}^{1}\right)$ and Path $2\left(S_{I}^{2} \rightarrow S_{F}^{2}\right)$, the resilient modulus obtained from the two stress path histories, either $M_{r}^{h}$ or $M_{r}^{v}$, are different (known in Chapter 4). But the stiffness anisotropy at final states $S_{F}^{1}$ and $S_{F}^{2}$ is identical when the ratios of $\sigma_{v} / \sigma_{h}$ at final stress states are the same.

$S_{I}^{1} \sim S_{I}^{4}$ and $S_{I}^{A} \sim S_{I}^{D}:$ Initial stress states

$S_{F}^{1} \sim S_{F}^{4}$ and $S_{F}^{A} \sim S_{F}^{D}$ : Final stress states

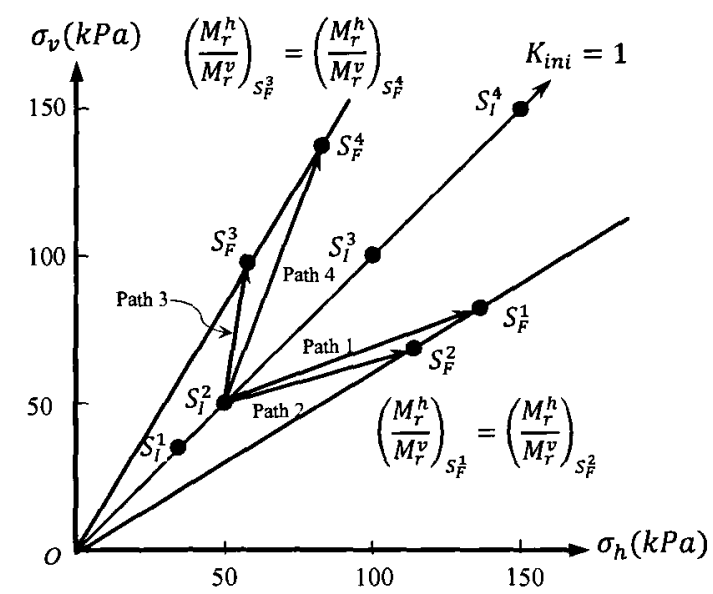

(a) Test series A (Isotropic consolidation)

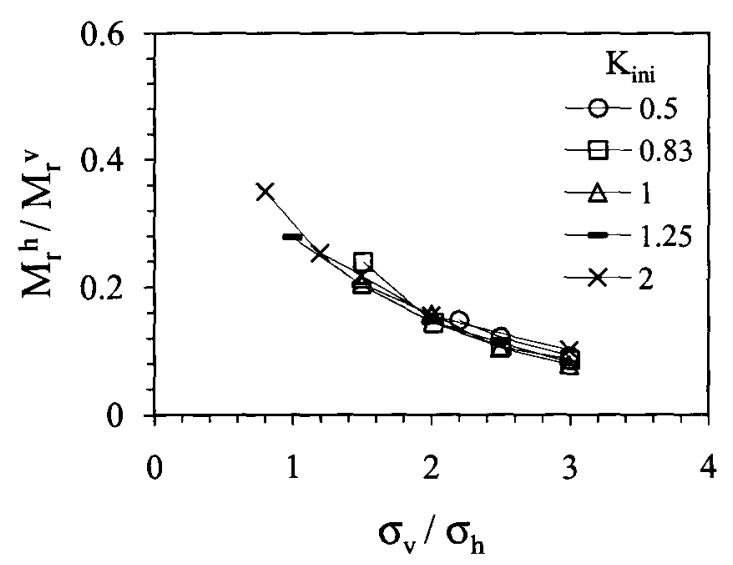

(c) Test Series B: $k=3.0$

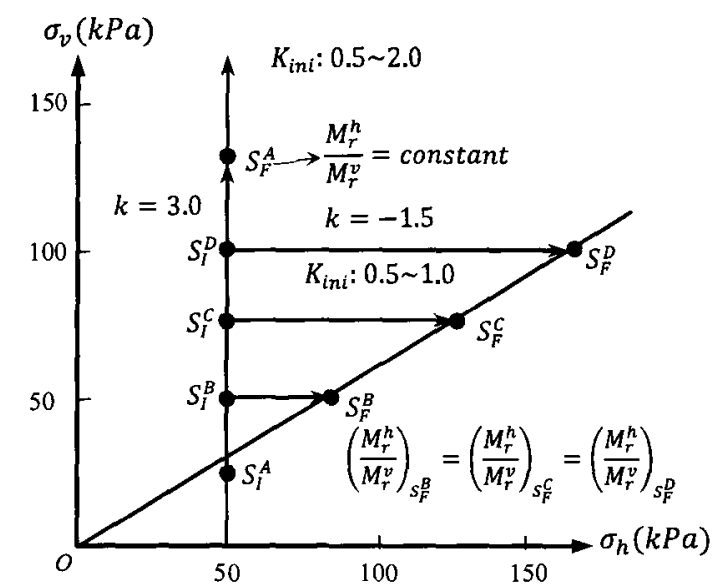

(b) Test series B (Anisotropic consolidation)

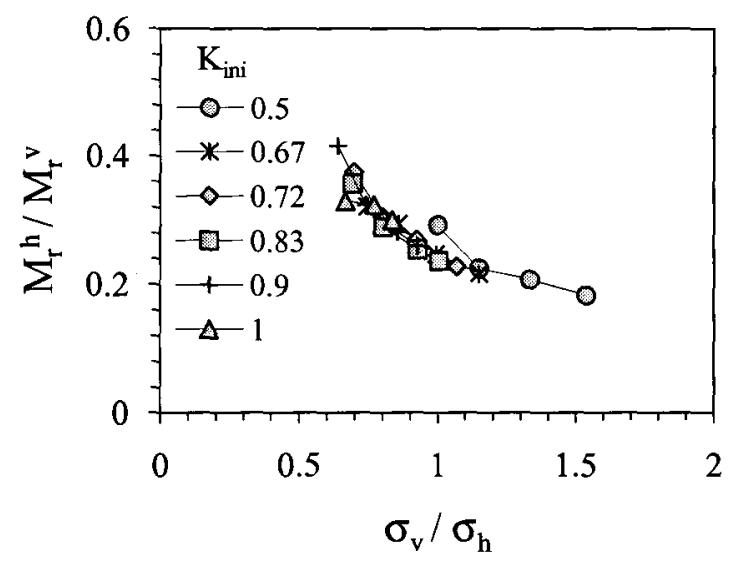

(d) Test Series B: $k=-1.5$

Fig. 5.12 Dependency of stiffness anisotropy on stress states 
Figures 5.12(b) through (d) present the variation of stiffness anisotropy with the stress ratio $\sigma_{v} / \sigma_{h}$ for test Series B. As shown in Figs. 5.12(b), $S_{I}^{A}, S_{I}^{B}, S_{I}^{C}$ and $S_{I}^{D}$ represent various initial anisotropic stress states, $S_{F}^{A}, S_{F}^{B}, S_{F}^{C}$ and $S_{F}^{D}$ represent various final stress states. When the sample is subjected to various stress path histories with $k=3.0$ and the same final stress states, such as $S_{I}^{A} \rightarrow S_{F}^{A}, S_{I}^{B} \rightarrow S_{F}^{A}, S_{I}^{C} \rightarrow S_{F}^{A}$ and $S_{I}^{D} \rightarrow S_{F}^{A}$, a higher resilient modulus, either in the vertical or in the horizontal direction, is obtained at lower $K_{\text {ini }}$ (see Fig. 5.4). However, the stiffness anisotropy obtained from these stress path histories is identical when the final stress states are the same, as shown in Fig. 5.12(c).

With respect to stress paths with $k=-1.5$ in test Series $\mathrm{B}$, different resilient moduli, either $M_{r}^{h}$ or $M_{r}^{v}$ are obtained from stress path histories $S_{I}^{B} \rightarrow S_{F}^{B}, S_{I}^{C} \rightarrow S_{F}^{C}$ and $S_{I}^{D} \rightarrow S_{F}^{D}$, but the same ratio of $\sigma_{v} / \sigma_{h}$ at the final states $S_{F}^{B}, S_{F}^{C}$ and $S_{F}^{D}$ also leads to identical stiffness anisotropy, see Figs. 5.8, 5.12(b) and (d).

Figures 5.12(c), (d) and 5.13 show that given an initial confining pressure $\sigma_{c}$ (strictly speaking, $\sigma_{h}^{i}$ ), the stress-induced anisotropy depends on $\sigma_{v} / \sigma_{h}$ at the final stress state, with $M_{r}^{h} / M_{r}^{v}$ increasing as $\sigma_{v} / \sigma_{h}$ decreases and being independent of $K_{\text {ini }}$. Furthermore, the relation of $\frac{M_{r}^{h}}{M_{r}^{v}}$ vs $\frac{\sigma_{v}}{\sigma_{h}}$ obtained from test Series B for various $K_{i n i}$ is very close to that of test Series A $\left(K_{i n i}=1\right)$ and follows a power law. Consequently, Eq. (4.29), which is used to predict the stress-induced stiffness anisotropy for initial isotropic stress states, can be used to evaluate the stress-induced stiffness anisotropy for both initial isotropic and anisotropic stress states by replacing the term $\sigma_{c}$ with $\sigma_{h}^{i}$; i.e., 


$$
\frac{M_{r}^{h}}{M_{r}^{v}}=m_{0}\left(\frac{\sigma_{h}^{i}}{p_{r}}\right)^{m_{c}}\left(\frac{\sigma_{v}}{\sigma_{h}}\right)^{m_{1}}
$$

Equation (5.1) suggests that the stress-induced stiffness anisotropy depends on the initial lateral confinement and subsequent $\sigma_{v} / \sigma_{h}$ ratio and is independent of $K_{i n i}$. It should be noted, $K_{i n i}$ may however have a significant influence on resilient modulus as presented in previous sections.

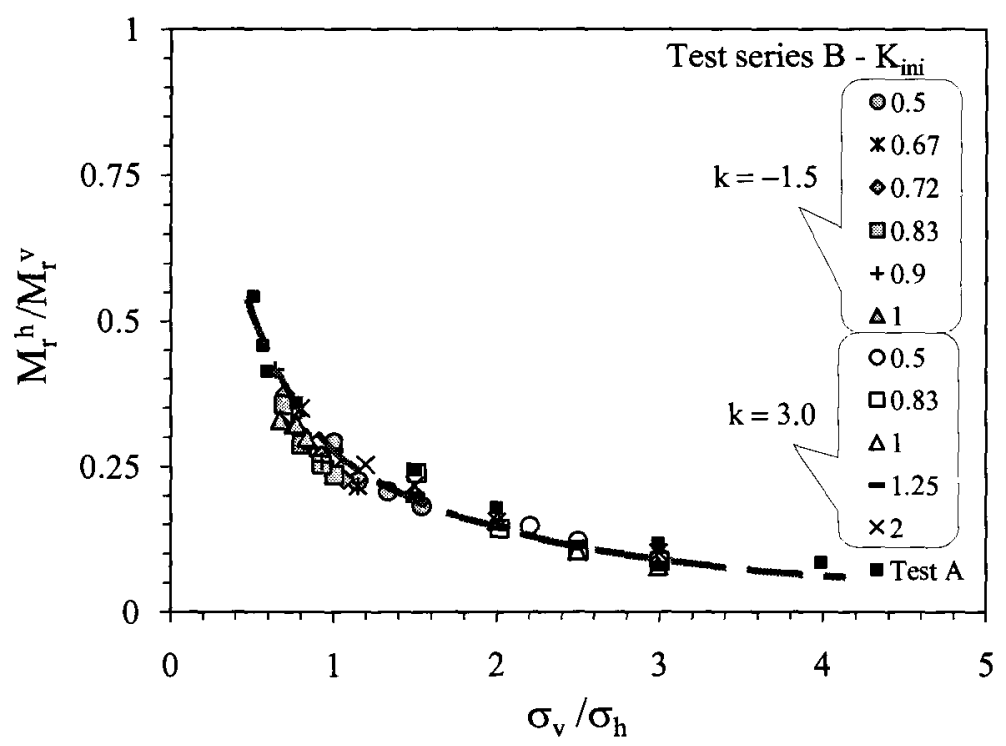

Fig. 5.13 Stress-induced stiffness anisotropy obtain from test Series A and B

$$
\sigma_{h}^{i}=50 \mathrm{kPa}
$$

\subsubsection{Initial anisotropy}

Similar to the derivation of the inherent stiffness anisotropy for an isotropic stress state in Chapter 4, the initial/inherent stiffness anisotropy for an anisotropic/isotropic initial stress state can be obtained from Eq. (5.1) by substituting the term $\sigma_{v} / \sigma_{h}$ at final stress states with ratio of $\sigma_{v}^{i} / \sigma_{h}^{i}$ (at initial stress state); i.e., $K_{\text {ini }}^{-1}$, therefore the initial stiffness 
anisotropy is given by

$$
\left(\frac{M_{r}^{h}}{M_{r}^{v}}\right)_{\text {initial }}=m_{0}\left(\frac{\sigma_{h}^{i}}{p_{r}}\right)^{m_{c}}\left(K_{\text {ini }}\right)^{-m_{1}}
$$

Equation (5.2) suggests that the initial stiffness anisotropy for an initial stress state depends on the initial lateral confinement and $K_{i n i}$, with $M_{r}^{h} / M_{r}^{v}$ increasing with $\sigma_{h}^{i}$ and $K_{\text {ini }}$. When $K_{\text {ini }}=1$, Eq. (5.2) becomes Eq. (4.30), which is used for predicting the inherent stiffness anisotropy at an isotropic stress state. It can be seen that the initial stiffness anisotropy depends on $K_{i n i}$, while the stress-induced stiffness anisotropy is independent of $K_{\text {ini }}$, but depends on the subsequent $\sigma_{v} / \sigma_{h}$ ratio.

\subsection{REVISED RESILIENT MODULUS EQUATION}

The $K_{i n i}$ effect on the resilient properties is incorporated now into the resilient modulus equations proposed in Chapter 4. The main ingredients of the equations are outlined as follows:

- $\sigma_{h}^{i}$ : the initial horizontal confinement on the soil sample or residual horizontal stress in the granular course.

- $K_{\text {ini }}$ : the ratio of $\sigma_{h} / \sigma_{v}$ at the initial stress state in the soil sample or the coefficient of earth pressure at rest.

- $\varepsilon_{h, r}$ and $\varepsilon_{v, r}$ : the resilient strains

Experiment evidence indicates that $K_{i n i}$ has no influence on $M_{r}^{h}$ if $M_{r}^{h}$ is modeled as a function of the horizontal resilient strains. Therefore, Eqs. (4.35) and (4.37) which 
characterize $M_{r}^{h}$ for stress paths with different principal stress directions, respectively, remain unchanged. Since the test results show that $M_{r}^{v}$ is greatly affected by $K_{\text {ini }}$, Eqs. (4.31) through (4.34) and (4.36) are modified by including the effect of $K_{i n i}$ on $M_{r}^{v}$ for stress paths with $k>0$ and $k<0$, respectively, via

$$
\begin{gathered}
\text { for } \varepsilon_{h, r}>0 \quad M_{r}^{v}=k_{1}\left(K_{i n i}\right)^{k_{01}}\left(\sigma_{h}^{i}\right)^{k_{2}}\left(\varepsilon_{h, r}^{2}+k_{3} \varepsilon_{h, r}+k_{4}\right) \\
\text { or } \\
\text { for } \varepsilon_{h, r}<0 \quad k_{1}^{\prime}\left(K_{i n i}\right)^{k_{o 2}}\left(\sigma_{h}^{i}\right)^{k_{2}^{\prime}} \varepsilon_{h, r}^{k_{3}^{\prime}} \\
M_{r}^{v}=k_{5}\left(K_{i n i}\right)^{k_{03}}\left(\sigma_{h}^{i}\right)^{k_{6}}\left(\varepsilon_{h, r}^{2}+k_{7} \varepsilon_{h, r}+k_{8}\right) \\
\text { or } \\
M_{r}^{v}=k_{5}^{\prime}\left(K_{i n i}\right)^{k_{o 4}}\left(\sigma_{h}^{i}\right)^{k_{6}^{\prime}}\left|\varepsilon_{h, r}\right|^{k_{7}^{\prime}} \\
M_{r}^{v}=k_{12}\left(K_{i n i}\right)^{k_{o 5}}\left(\sigma_{h}^{i}\right)^{k_{13}} \exp ^{k_{14} \varepsilon_{v, r}}
\end{gathered}
$$

where $k_{1} \sim k_{8}, k_{12} \sim k_{14}, k_{1}^{\prime} \sim k_{3}^{\prime}, k_{5}^{\prime} \sim k_{7}^{\prime}$, and $k_{o 1} \sim k_{o 5}$ are regression constants. Equations (5.3) through (5.6) are for stress paths $k>0$, and Eq. (5.7) is for stress paths with $k<0$.

Equations (5.5) through (5.7) are then used to fit the measured $M_{r}^{v}$ for the various tests of Series B, respectively. Throughout test Series B, a single initial lateral stress of 50 $\mathrm{kPa}$ was applied, so the terms $\left(\sigma_{h}^{i}\right)^{k_{6}},\left(\sigma_{h}^{i}\right)^{k_{6}^{\prime}}$, and $\left(\sigma_{h}^{i}\right)^{k_{13}}$ in the equations above are omitted and merged into terms $k_{5}, k_{5}^{\prime}$ and $k_{12}$, respectively. Figure 5.14 compares the predicted and measured resilient moduli. The figures confirm that Eqs. (5.5) through (5.7) provide good fit to the measured resilient moduli and can be used to estimate $M_{r}^{v}$ with confidence for stress paths with $k \neq 0$, respectively. 

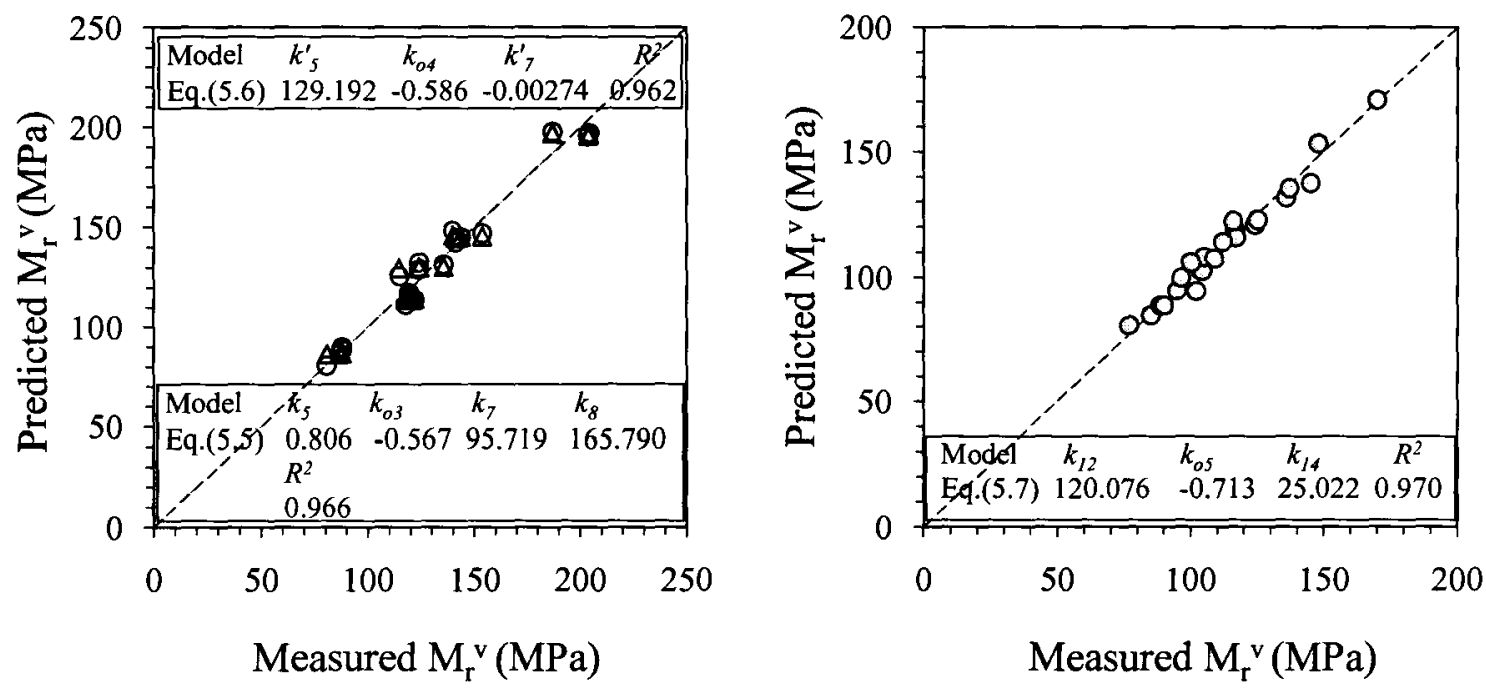

Fig. 5.14 Comparison between predicted and measured resilient moduli

It is noted that the coefficient, $k_{o 5}$, which quantifies the effect of $K_{i n i}$ on $M_{r}^{v}$ for stress paths with $k=-1.5$ is smaller than $k_{o 3}$ and $k_{o 4}$ for $k=3.0$. This implies that larger $K_{i n i}$ value can reduce $M_{r}^{v}$ for stress paths with $k<0$ more rapidly than that for stress paths with $k>0$. As such, larger $K_{i n i}$ has an adverse effect on $M_{r}^{v}$, and great care must be taken when selecting the parameters of $M_{r}^{v}$ for design. This is particularly true for the areas of critical location and those where the major principal stress is in the horizontal direction.

\subsection{RESILIENT POISSON'S RATIO}

The general variations of $v_{v h}$ and $v_{h h}$ with $\sigma_{v} / \sigma_{h}$ and $K_{i n i}$ for stress paths $k=3.0$ and -1.5 , are presented in Figs. 5.15 and 5.16, respectively. In addition to the observation that both $v_{v h}$ and $v_{h h}$ increase with an increase in $\sigma_{v} / \sigma_{h}$, it is observed that at a given ratio of $\sigma_{v} / \sigma_{h}, v_{v h}$ and $v_{h h}$ decrease as $K_{i n i}$ decreases. 

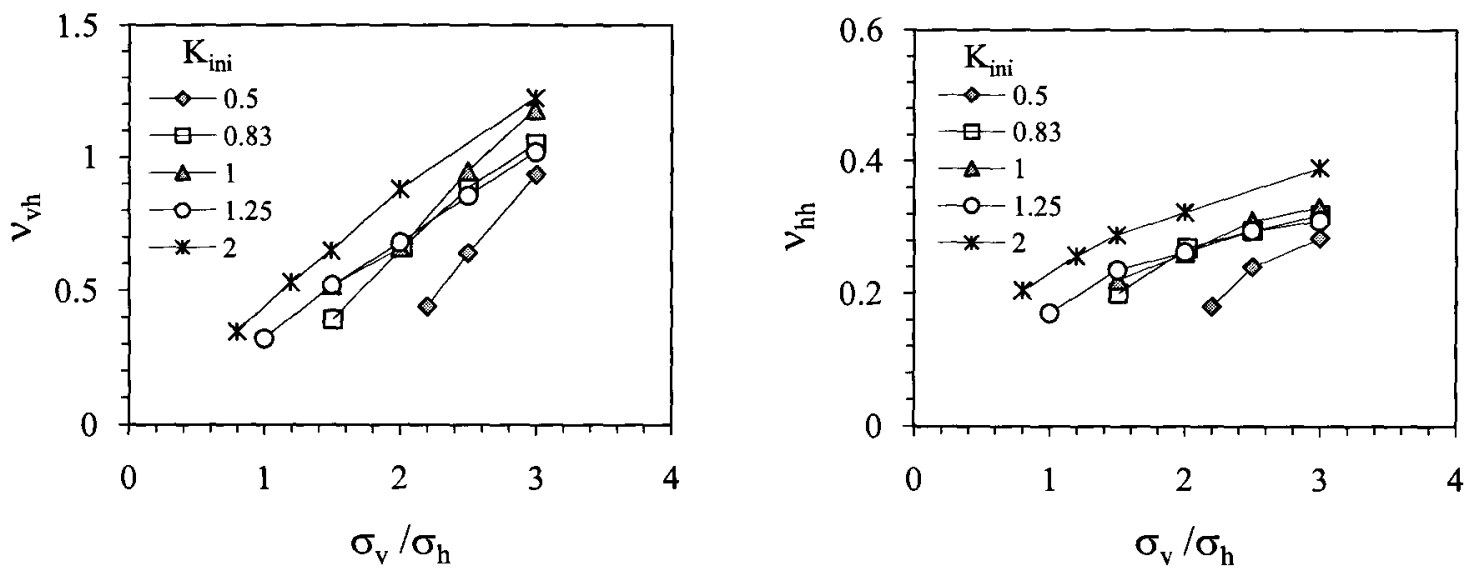

Fig. 5.15 Variation of Poisson's ratios with $K_{i n i}$ for $k=3.0$

$\sigma_{c}=50 \mathrm{kPa}$
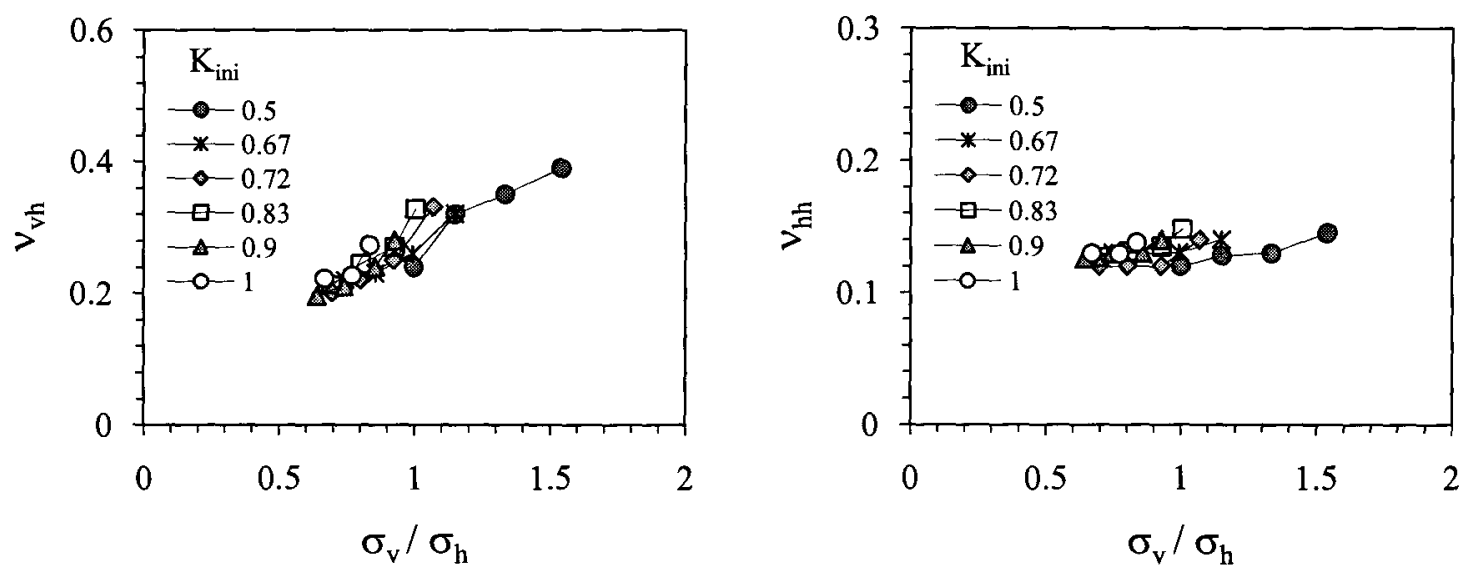

Fig. 5.16 Variation of Poisson's ratios with $K_{\text {ini }}$ for $k=-1.5$

$$
\sigma_{h}^{i}=50 \mathrm{kPa}
$$

The Poisson's ratios for stress paths with $k=3.0$ are influenced more by $K_{i n i}$ than those for stress paths with $k=-1.5$. This may be attributed to the nature of disposition of soil or the direction of the bedding plane. The dependency of Poisson's ratio on $K_{i n i}$ reflects the influence of initial stress state, which in turn affects the resilient deformation under subsequent cyclic loading. For example, the resistance of deformation to the 
applied load for $k=3.0$ in both the vertical and horizontal directions increases as $K_{\text {ini }}$ decreases, as shown in Fig. 5.2. Consequently, $v_{v h}$ and $v_{h h}$ decrease as $K_{i n i}$ decreases.

It is noted that the variations of $v_{v h}$ and $v_{h h}$ with $K_{i n i}$ at a given ratio of $\sigma_{v} / \sigma_{h}$ for stress paths with $k=3.0$ are wider than those for stress paths with $k=-1.5$. These findings are consistent with those presented in Chapter 4. Since higher $\sigma_{v} / \sigma_{h}(k=3.0)$ may induce resilient dilatancy and the material tends to become more anisotropic, which in turn yield higher $v_{v h}$ and $v_{h h}$. Moreover, the material tends to become less anisotropic when $k=-1.5$. As a result, the variations of $v_{v h}$ and $v_{h h}$ for stress paths with $k=-1.5$ are smaller than those for $k=3.0$.

A suggestion is made in Chapter 4 on the selection of resilient Poisson's ratios for design of flexible pavement. Based on the scrutiny of the data and the analysis in this chapter, Eqs. (4.42) and (4.43) need to be modified to provide an approximation for $v_{v h}$ and $v_{h h}$ for stress paths with the onset of obvious extensive $\varepsilon_{h, r}$ for various $K_{i n i}$ values. The modified formulations are given by

$$
\begin{aligned}
& v_{v h}=c_{0} K_{i n i}{ }^{c_{K}}\left(\left(\frac{\sigma_{v}}{\sigma_{h}}\right)^{2}+c_{1}\left(\frac{\sigma_{v}}{\sigma_{h}}\right)+c_{2}\right) \\
& v_{h h}=d_{0} K_{i n i}{ }^{d_{K}}\left(\left(\frac{\sigma_{v}}{\sigma_{h}}\right)^{2}+d_{1}\left(\frac{\sigma_{v}}{\sigma_{h}}\right)+d_{2}\right)
\end{aligned}
$$

where $c_{0} \sim c_{2}, d_{0} \sim d_{2}, c_{K}$ and $d_{K}$ are regression constants.

Figure 5.17 shows the curve fitting results from test data for various $K_{i n i}$ at $k=3.0$. The figure demonstrates that the proposed Eqs. (5.8) and (5.9) are capable of modelling the resilient Poisson's ratios for various $K_{\text {ini }}$ conditions along a stress path, where 
extensive radial strains are induced. The values of $c_{K}$ and $d_{K}$ obtained from regression analysis reveal that Eqs. (5.8) and (5.9) can be approximated as a linear function of $\sigma_{v} / \sigma_{h}$ without loss of much accuracy of the predications.

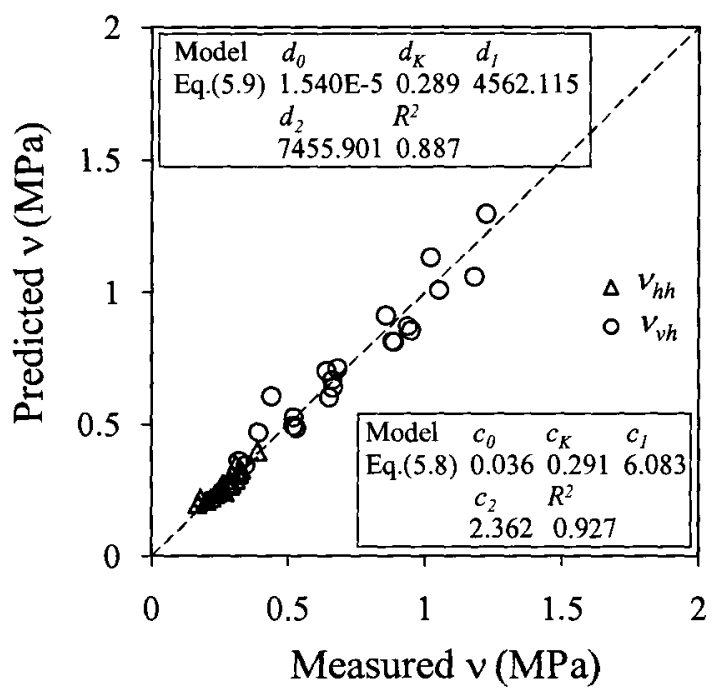

Fig. 5.17 Predicted and measured Poisson's ratios for $k=3.0$

\subsection{SUMMARY}

This chapter investigated the anisotropic resilient behaviour of the test material for various initial anisotropic stress states for stress paths with $k=3.0$ and -1.5 . Although the variation in void ratio is small among the various cases, the resilient response is influenced by $K_{i n i}$. In particular, $M_{r}^{v}$ is more influenced by $K_{i n i}$ than $M_{r}^{h}$. When expressed as a function of resilient strain, $M_{r}^{v}$ increases with a decrease in $K_{i n i}$ when $\varepsilon_{v, r}$ is the same. $M_{r}^{h}$, on the other hand, is independent of $K_{i n i}$ when expressed as a function of $\varepsilon_{h, r}$. The test results clearly show that the stiffness anisotropy is stress-state dependent. It is however interesting to note that the stress-induced stiffness anisotropy is independent of 
$K_{i n i}$, but relies on the initial lateral confinement and subsequent $\sigma_{v} / \sigma_{h}$ ratio. On the other hand, the initial stiffness anisotropy depends on the initial lateral confinement and $K_{\text {ini }}$.

The resilient modulus equations proposed in Chapter 4 were revised to incorporate the effect of $K_{i n i}$ on the resilient modulus, in particular $M_{r}^{v}$. Comparison between measured and predicted resilient moduli showed that the revised equations are capable of describing the resilient properties for various $K_{i n i}$ for different stress paths.

The resilient Poisson's ratios, $v_{v h}$ and $v_{h h}$ were found to be influenced by $K_{i n i}$, with the Poisson's ratios being more influenced for stress paths having $k>0$ than those having $k<0$. For a given initial confinement, $v_{v h}$ and $v_{h h}$ decrease with a decrease in $K_{i n i}$ when $\sigma_{v} / \sigma_{h}$ is the same. The resilient Poisson's ratio equations suggested in Chapter 4 were modified to account for the effect of $K_{i n i}$. It was shown that the modified equations give a good fit to the measured data from the stress path tests. 


\section{ANISOTROPY WITHIN MICROMECHANICAL FRAMEWORK}

\subsection{FABRIC ANISOTROPY: MICROMECHANICS}

\subsubsection{Introduction}

The overall mechanical behaviour of a granular material, which is a collection of discrete particles, is significantly affected by its microstructure, including geometrical arrangement of particles (i.e., fabric) and the interaction at particle contacts. The inherent anisotropic characteristics of granular materials are mostly induced by the mode of deposition or compaction, which results in different particle arrangement and interparticle locking.

When subjected to deviator stress, soil particles move around as the soil develops shear resistance. The relative movement between particles causes particle rearrangement, which in turn results in fabric change that is termed as induced anisotropy. Therefore, anisotropic fabric and anisotropic stresses lead to the anisotropic resilient behaviour of granular materials. Fabric anisotropy should therefore be taken into account in interpreting the experimental data and understanding the resilient behaviour of granular materials. This allows one to get more comprehensive insight into the resilient properties of granular materials under conditions of isotropic or anisotropic consolidation along different stress/strain paths. Furthermore, a quantitative description of evolution of fabric anisotropy, which can be back-calculated from the test results, can advance the elastic models based on micromechanics and provide a reliable prediction of initial fabric 
anisotropy and fabric evolution in constitutive models with embedded microstructure.

\subsubsection{Fundamentals: Description of anisotropic microstructure}

The fabric anisotropy (the directional distribution of granular assembly) can be characterized by different elements of the microstructure, such as the distribution of particle contact normals, branch vectors, particle orientation and void orientation (Oda et al., 1985). Herein contact normal $\boldsymbol{n}$ is defined as a vector normal to the tangent plane at a point of a contact between particles, and the branch vector $l$ is represented by a line joining the centroid of contacting particles, as shown in Fig. 6.1.

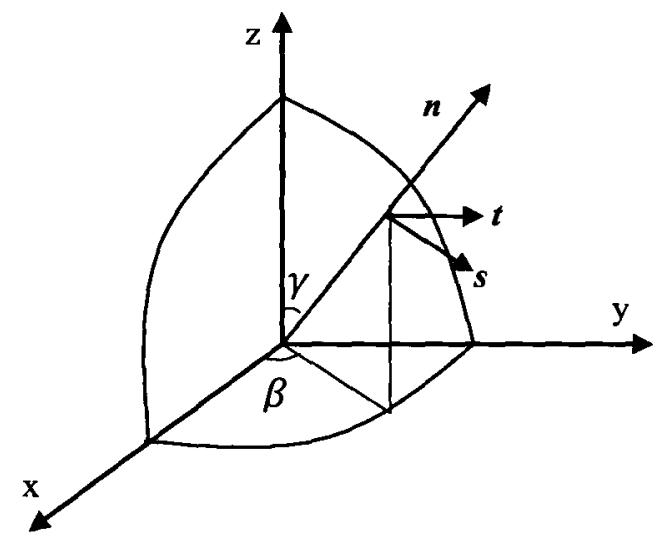

(a)

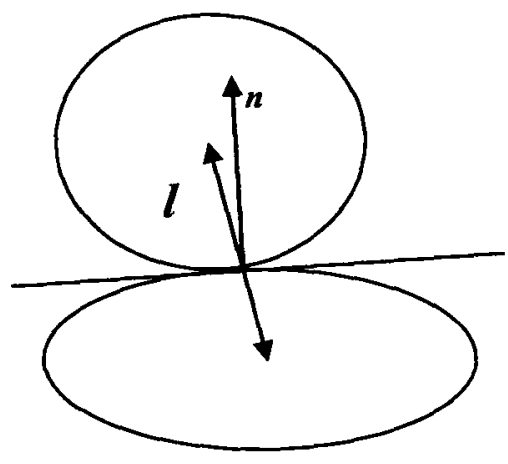

(b)

Fig. 6.1 (a) Local coordinate at inter particle contact (b) Definition of branch vector

In order to quantitatively define fabric anisotropy, a fabric tensor $F_{i j}$ is introduced by averaging the directional distribution of branch vector over a representative elementary volume (REV) as

$$
F_{i j}=\frac{N}{V} \int E(\boldsymbol{n}) l_{i} l_{j} d \Omega
$$


where $l_{i}$ is the branch vector, $N$ is the number of contacts in the assembly, $V$ is the volume of the assembly, $E(\boldsymbol{n})$ is a spatial probability density function of contact normals $n, \Omega$ is the unit sphere, and $d \Omega$ is the solid angle of a sphere, satisfying $d \Omega=\sin \gamma d \gamma d \beta$.

For cross-anisotropic fabric with its symmetry along the $z$-axis (Fig. 6.1), a truncated Fourier series can be used as an approximation to describe the contact normal distribution function; i.e.,

$$
E(\gamma, \beta)=\frac{1}{4 \pi}\left(1+\frac{a}{4}(3 \cos (2 \gamma)+1)\right)
$$

where $a$ is the degree of fabric anisotropy, a quantity used to describe the packing anisotropy of materials (Chang and Mistra, 1990).

Figure 6.2 shows an example of contact normal distribution with different values of a. A value of $a=0$ represents an isotropic distribution of the contact normals. When $a>0$, the contact normals of the particles tend to concentrate in the vertical direction, whereas when $a<0$, the contact normals tend to concentrate in the horizontal directions.

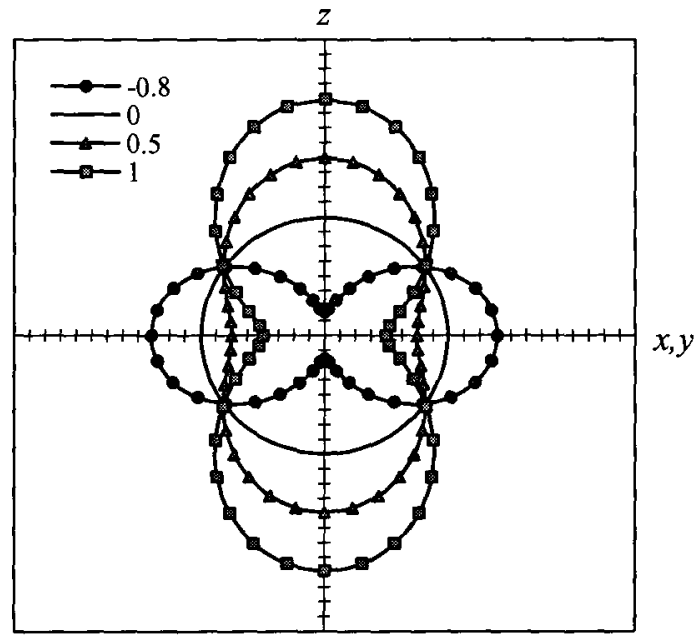

Fig. 6.2 Distribution of contact orientations with different value of fabric anisotropy 


\subsubsection{Macro-Micro relationship}

The stress-strain relation for an assembly can be determined from integrating the behaviour of interparticle contacts in all orientations. For an assembly of particles enclosed in a RVE of volume $V$ with $N$ contacts, the increment of mean force on the contact plane can be expressed as

$$
d f_{i}=d \sigma_{i j} A_{j k} l_{k}
$$

where $A_{j k}=\left(F_{j k}\right)^{-1}$, and $d \sigma_{i j}$ is the macroscopic stress increment, which can be obtained from the contact forces and branch vectors for contacts in all orientations, using

$$
d \sigma_{i j}=\frac{N}{V} \int l_{i} d f_{j} E(\boldsymbol{n}) d \Omega
$$

The macroscopic strain increment $d \varepsilon_{i j}$ can be calculated from the interparticle displacement increment $d u_{i}$, given as

$$
d \varepsilon_{i j}=\frac{N}{V} \int l_{n} d u_{i} A_{j n} E(\boldsymbol{n}) d \Omega
$$

Equations (6.1) and (6.3) through (6.5) are basic formulations, from which the compliance matrix can be derived for an assembly of particles with a fabric tensor $F_{i j}$.

\subsection{A COMPLIANCE MATRIX FOR GRANULAR ASSEMBLY}

According to the contact law, the interparticle displacement increment can be related to the contact force and contact stiffness, via

$$
d u_{j}=M_{i j} d f_{i}
$$


where $M_{i j}$ is a flexibility tensor. Assuming that the contact stiffness between the normal and shear direction is uncoupled, the flexibility tensor can be written as

$$
M_{i j}=\frac{1}{k_{n}} n_{i} n_{j}+\frac{1}{k_{s}} s_{i} s_{j}+\frac{1}{k_{s}} t_{i} t_{j}
$$

where $k_{n}$ and $k_{s}$ are contact normal stiffness and shear/tangential stiffness, respectively. For each contact particle, the local coordinate system is related to the global coordinate system according to Table 6.1.

Table 6.1 The local to global coordinate system

\begin{tabular}{cccc}
\hline Element & $\boldsymbol{n}$ & $\boldsymbol{s}$ & $\boldsymbol{t}$ \\
\hline 1 & $\cos \gamma$ & $\sin \gamma$ & 0 \\
\hline 2 & $\sin \gamma \cos \beta$ & $\cos \gamma \cos \beta$ & $\sin \beta$ \\
\hline 3 & $\sin \gamma \sin \beta$ & $\cos \gamma \sin \beta$ & $\cos \beta$ \\
\hline
\end{tabular}

Substituting Eqs. (6.3) and (6.6) into Eq. (6.5), the macroscopic stress-strain relationships can be written as

$$
d \varepsilon_{i j}=C_{i j k l} d \sigma_{k l}
$$

where $C_{i j k l}$ is expressed as

$$
C_{i j k l}=\frac{V}{N} \int_{0}^{2 \pi} \int_{0}^{\pi} M_{i k} l_{n} l_{q} A_{j n} A_{l q} E(\gamma, \beta) \sin \gamma d \gamma d \beta
$$

with $A_{j n}{ }^{-1}=\frac{N}{V} \int l_{j} l_{n} E(l) d \Omega$. For a cross-anisotropic fabric, $A_{i j}=0$, when $i \neq j$.

At this point it is assumed that the granular assembly consists of a large number of sphere particles with radius $R$, and all particles have the same material properties. Given Eq. (6.2), a closed form stress-strain relation can be derived from Eqs. (6.8) and (6.9). 
The compliance matrix for a cross-anisotropic material can be derived after performing integrations. The elastic matrix for a cross-anisotropic material becomes

$$
\left(\begin{array}{c}
\Delta \varepsilon_{z z} \\
\Delta \varepsilon_{x x} \\
\Delta \varepsilon_{y y} \\
\Delta \gamma_{x z} \\
\Delta \gamma_{y z} \\
\Delta \gamma_{x y}
\end{array}\right)=\left[\begin{array}{cccccc}
B_{11} & B_{12} & B_{13} & 0 & 0 & 0 \\
B_{21} & B_{22} & B_{23} & 0 & 0 & 0 \\
B_{31} & B_{32} & B_{33} & 0 & 0 & 0 \\
0 & 0 & 0 & B_{44} & 0 & 0 \\
0 & 0 & 0 & 0 & B_{55} & 0 \\
0 & 0 & 0 & 0 & 0 & B_{66}
\end{array}\right]\left[\begin{array}{c}
\Delta \sigma_{z z} \\
\Delta \sigma_{x x} \\
\Delta \sigma_{y y} \\
\Delta \tau_{x z} \\
\Delta \tau_{y z} \\
\Delta \tau_{x y}
\end{array}\right]
$$

with

$$
\begin{aligned}
& B_{11}=\frac{1}{M_{r}^{v}}=\frac{15 V}{28 R^{2} N(5+2 a)^{2}}\left(\frac{1}{k_{n}}(21+12 a)+\frac{1}{k_{s}}(14+2 a)\right) \\
& B_{22}=B_{33}=\frac{1}{M_{r}^{h}}=\frac{15 V}{28 R^{2} N(5-a)^{2}}\left(\frac{1}{k_{n}}(21-6 a)+\frac{1}{k_{s}}(14-a)\right) \\
& B_{12}=B_{21}=\frac{-v_{v h}}{M_{r}^{v}}=\frac{-v_{h v}}{M_{r}^{h}}=\frac{15 V(7+a)}{28 R^{2} N(5+2 a)(5-a)}\left(\frac{1}{k_{n}}-\frac{1}{k_{s}}\right) \\
& B_{23}=B_{32}=\frac{-v_{h h}}{M_{r}^{h}}=\frac{15 V(7-2 a)}{28 R^{2} N(5+2 a)(5-a)}\left(\frac{1}{k_{n}}-\frac{1}{k_{s}}\right) \\
& B_{44}=\frac{1}{G_{r}^{v h}}=\frac{15 V(10+a)}{28 R^{2} N(5+2 a)^{2}(5-a)^{2}}\left(\frac{1}{k_{n}}\left(a^{2}+17 a+70\right)\right. \\
& \left.-\frac{1}{k_{s}}\left(15 a^{2}+18 a+105\right)\right) \\
& B_{55}=B_{66}=\frac{1}{G_{r}^{h h}}=\frac{15 V}{14 R^{2} N(5-a)^{2}}\left(\frac{1}{k_{n}}(14-4 a)+\frac{1}{k_{s}}(21-3 a)\right)
\end{aligned}
$$

Defining $r=\frac{k_{s}}{k_{n}}$, the elastic parameters for cross-anisotropic materials can be obtained as follows 


$$
\begin{gathered}
M_{r}^{v}=\frac{28 R^{2} N(5+2 a)^{2} k_{n} r}{15 V(14+2 a+r(21+12 a))} \\
M_{r}^{h}=\frac{28 R^{2} N(5-a)^{2} k_{n} r}{15 V(14-a+r(21-6 a))} \\
v_{v h}=\frac{(5+2 a)(7+a)(1-r)}{(5-a)(14+2 a+r(21+12 a))} \\
v_{h h}=\frac{(7-2 a)(1-r)}{(14-a+r(21-6 a))} \\
\frac{28 R^{2} N(5+2 a)^{2}(5-a)^{2} k_{n} r}{15 V(10+a)}\left(-15 a^{2}+18 a+105\right. \\
+r(10+a)(7+a)) \\
G_{r}^{h h}=\frac{14 R^{2} N(5-a)^{2} k_{n} r}{15 V(21-3 a+r(14-4 a))}
\end{gathered}
$$

The resilient modulus ratios then become

$$
\begin{gathered}
\frac{M_{r}^{h}}{M_{r}^{v}}=\frac{(5-a)^{2}(14+2 a+r(21+12 a))}{(5+2 a)^{2}(14-2 a+r(21-6 a))} \\
\frac{G_{r}^{h h}}{G_{r}^{v h}}=\frac{(10+a)\left(-15 a^{2}+18 a+105+r(10+a)(7+a)\right)}{(5+2 a)^{2}(21-3 a+r(14-4 a))}
\end{gathered}
$$

A more detailed derivation is included in Appendix III. Yimsiri and Soga (2000) derived a similar matrix with a different contact normal density function to study the small strain behaviour of sand and clay, which is assumed to have constant fabric anisotropy and constant $k_{s} / k_{n}$.

The effect of fabric anisotropy on resilient properties can be investigated using Eqs. (6.12) through (6.19). Figure 6.3 shows the variation of modulus ratio over a range of 
degree of fabric anisotropy for various $k_{s} / k_{n}$ based on Eq. (6.18). It demonstrates that $a$ has a primary influence on the stiffness anisotropy, and $k_{s} / k_{n}$ has a secondary influence on stiffness anisotropy. At a given ratio of $k_{s} / k_{n}, M_{r}^{h} / M_{r}^{v}$ decreases as $a$ increases. When $a>0$, which implies a material has a stronger fabric in the vertical direction than the horizontal direction, the effect of $k_{s} / k_{n}$ on stiffness anisotropy, is smaller than that with a negative value of $a$. When a material becomes more anisotropic, no matter $a>0$ or $a<0$, the effect of $k_{s} / k_{n}$ on the stiffness anisotropy tends to become larger.

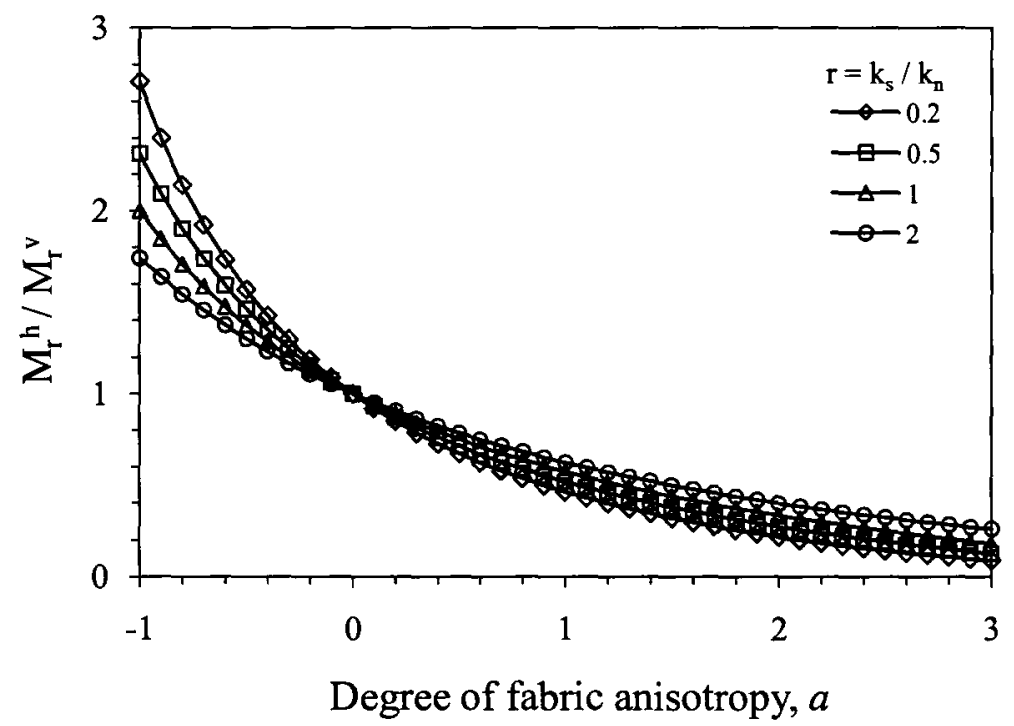

Fig. 6.3 Variation of stiffness ratio with degree of fabric anisotropy

Figure 6.4 shows the influence of $k_{s} / k_{n}$ and $a$ on the resilient Poisson's ratios based on Eqs. (6.14) and (6.15). As can be seen from this figure, $v_{v h}=0$ when $=-2.5$, which can be considered as the transitional value for $a$. For a given degree of fabric anisotropy with $a>-2.5, v_{v h}$ decreases with an increase in $k_{s} / k_{n}$ as a result of increased constraint to particle movement in the tangential plane at any contact point. 
With respect to $v_{h h}$, the transitional value of $a$ is 3.5 ; i.e., $v_{h h}=0$ when $a=3.5$. When $a<3.5, v_{h h}$ decreases with an increase in the ratio of $k_{s} / k_{n}$.
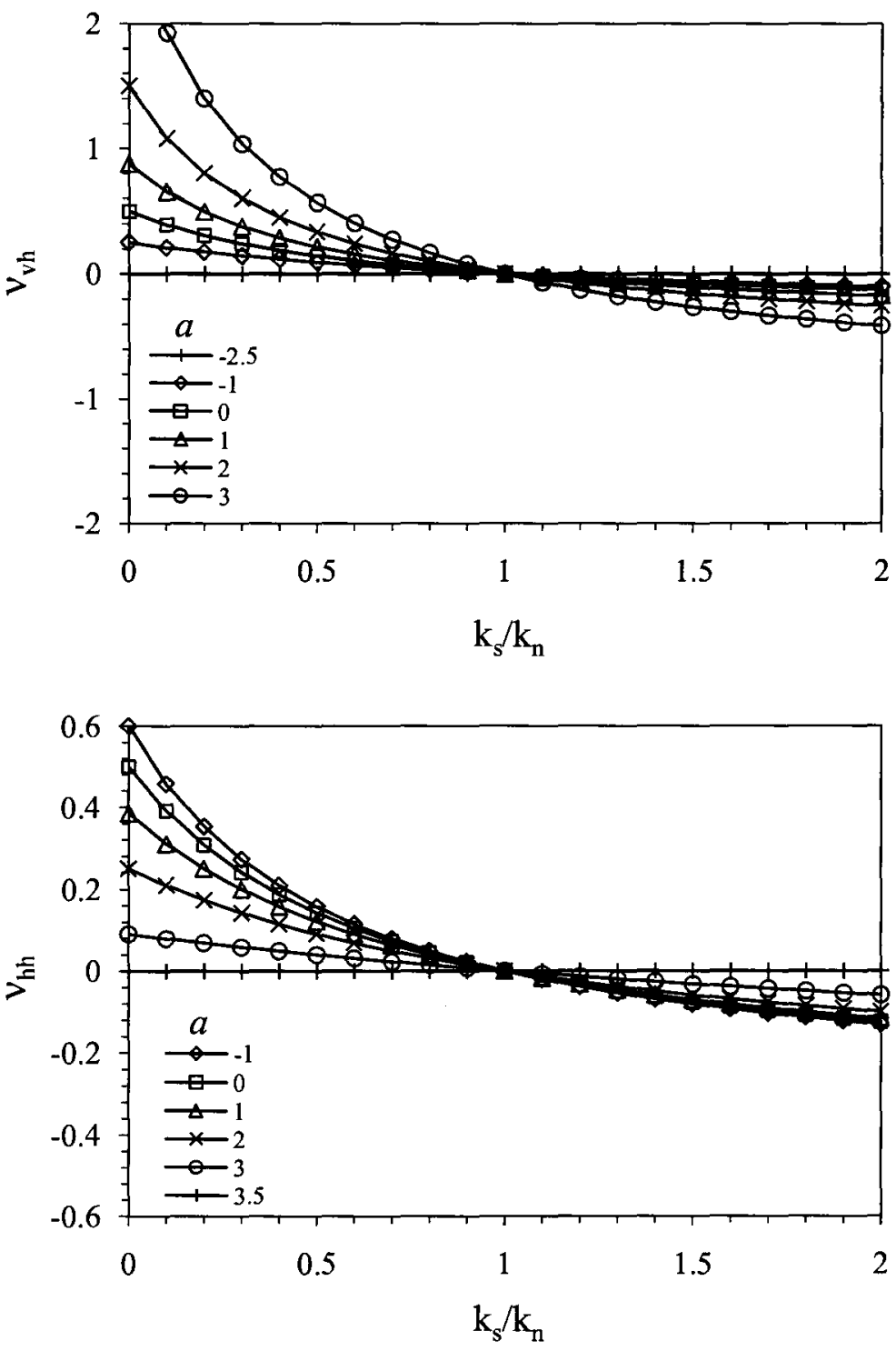

Fig. 6.4 Variation of Poisson's ratios

Figure 6.4 also shows that the degree of fabric anisotropy $a$ has different influences on $v_{v h}$ and $v_{h h}$. When $k_{s} / k_{n}<1, v_{v h}$ increases as the degree of fabric anisotropy 
increases; while $v_{h h}$ decreases as degree of fabric anisotropy increases. When $k_{s} / k_{n}>1$, the trends of $v_{v h}$ and $v_{h h}$ become opposite. For isotropic materials with $a=0, v_{v h}=$ $v_{h h}$. When $k_{s} / k_{n}<1$, for materials with $a>0$. (i.e., more contact normals concentrate in the vertical direction), $v_{v h}>v_{h h}$, while $v_{v h}<v_{h h}$ for materials with $a<0$. It should be noted that $k_{s} / k_{n}$ is usually less than unity for most granular materials.

\subsection{PREDICTION OF $v_{h h}, M_{r}^{h}$ AND $G_{r}^{v h}$}

The micromechanics model presented in the previous section clearly shows that the elastic parameters can be related to 2 major microscopic quantities of the material; i.e., fabric anisotropy and contact properties. As such, the test results of the resilient moduli obtained from stress path tests in this study can be used to back-calculate the "effective" $a$ and $k_{s} / k_{n}$ with numerical optimization method. For each stress path test, Eqs. (6.14) and (6.18) are used to determine $a$ and $k_{s} / k_{n}$.

It is important to recognize that some elastic parameters that cannot be obtained from triaxial tests for a cross-anisotropic material, such as the vertical resilient shear modulus $G_{r}^{v h}$, can be determined using micromechanics formulations.

As discussed in Chapter 4, when solving a system of linear Eq. (4.23), $v_{h h}$ and $M_{r}^{h}$ are always coupled. The Graham-Houlsby assumption; i.e., Eq. (4.25) was used to decouple $v_{h h}$ and $M_{r}^{h}$ in $\frac{1-v_{h h}}{M_{r}^{h}}$. However, $v_{h h}$ and $M_{r}^{h}$ can be obtained using the micromechanics model without using the Graham-Houlsby assumption. From Eq. (4.19), the following equation can be obtained 


$$
\frac{1-v_{h h}}{M_{r}^{h}} \frac{M_{r}^{v}}{v_{v h}}=\frac{\Delta \sigma_{v}}{\Delta \sigma_{h}}+\frac{\varepsilon_{h, r}}{\Delta \sigma_{h}} \frac{M_{r}^{v}}{v_{v h}}
$$

Equations (6.14), (6.15) and (6.18) are then substituted into Eq. (6.20), which becomes

$$
\frac{(5+2 a)(7+a+4 r(7-2 a))}{(5-a)(7+a)(1-r)}=\frac{\Delta \sigma_{v}}{\Delta \sigma_{h}}+\frac{\varepsilon_{h, r}}{\Delta \sigma_{h}} \frac{M_{r}^{v}}{v_{v h}}
$$

As a result, $a$ and $k_{s} / k_{n}$ ratio can be solved from Eqs.(6.14) and (6.21). Therefore, $v_{h h}$ and $M_{r}^{h}$ can be calculated from Eqs. (6.15) and (6.18).

Figure 6.5 compares $a$ and $k_{s} / k_{n}$ calculated from Eqs. (6.14) and (6.18) with those from Eqs. (6.14) and (6.21). It demonstrates that $a$ and the corresponding $k_{s} / k_{n}$ calculated from both approaches are very close. Figure 6.6 compares $M_{r}^{h}$ and $v_{h h}$ obtained from the micromechanics and the Graham-Houlsby approaches, respectively. As can be seen from this figure, the horizontal moduli $M_{r}^{h}$ calculated from both approaches are in good agreement. Therefore, both the micromechanical approach and GrahamHoulsby assumption can be used to calculate $M_{r}^{h}$ with confidence. The values of $v_{h h}$ from micromechanics model are however systematically smaller than those based on the Graham-Houlsby assumption. The outliers in data of $v_{h h}$ are mostly for stress paths with $k=2.0$, as discussed in Section 4.4.2, the level of measured $\varepsilon_{h, r}$ is very small $(\sim 0.001 \%)$ compared to $\varepsilon_{v, r}$, which in turn could lead to round off errors when determining the corresponding parameters. The differences in $v_{h h}$ should be noted when using these 2 approaches to evaluate the values of $v_{h h}$. Both approaches are not perfect as assumptions (sphere particles and Eq. (4.25), respectively) are made when evaluating $M_{r}^{h}$ and $v_{h h}$. 

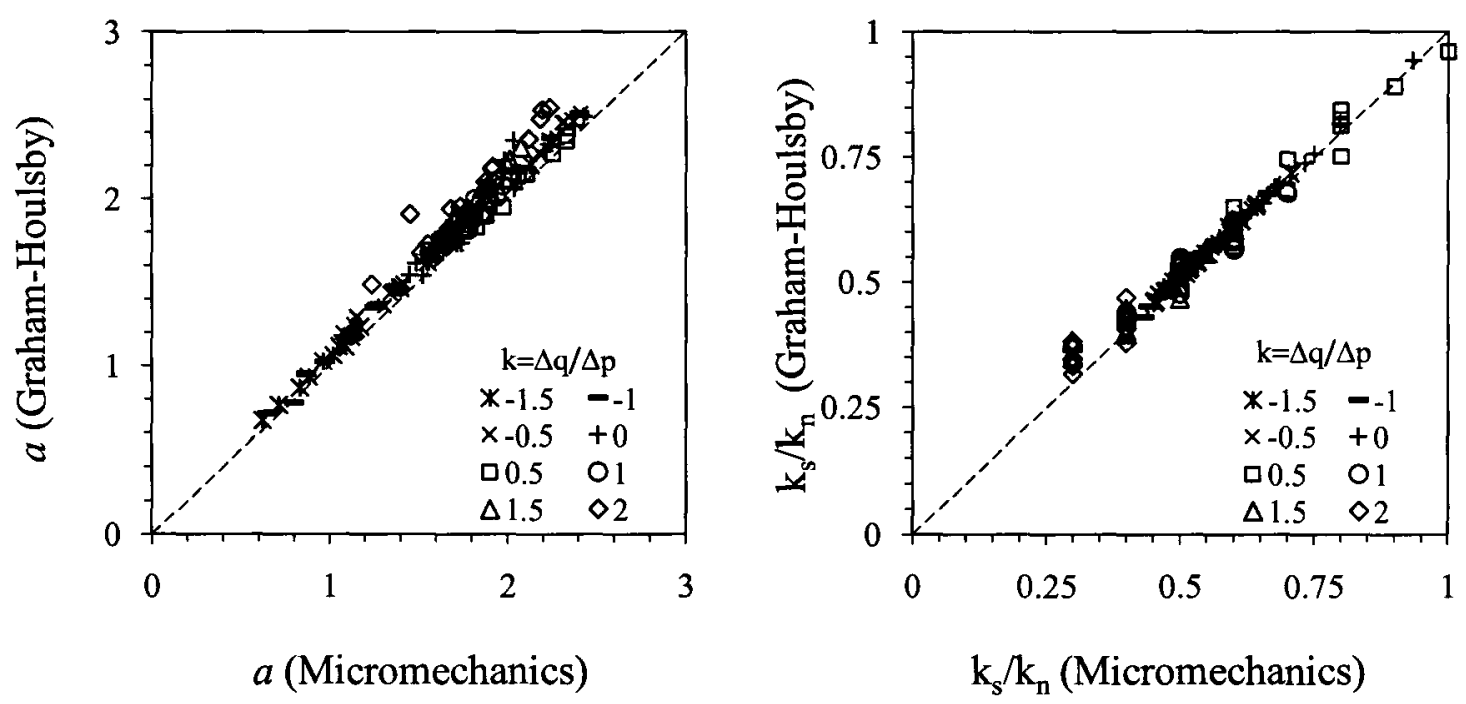

Fig. $6.5 a$ and $k_{s} / k_{n}$ obtained from micromechanics model and G-H assumption
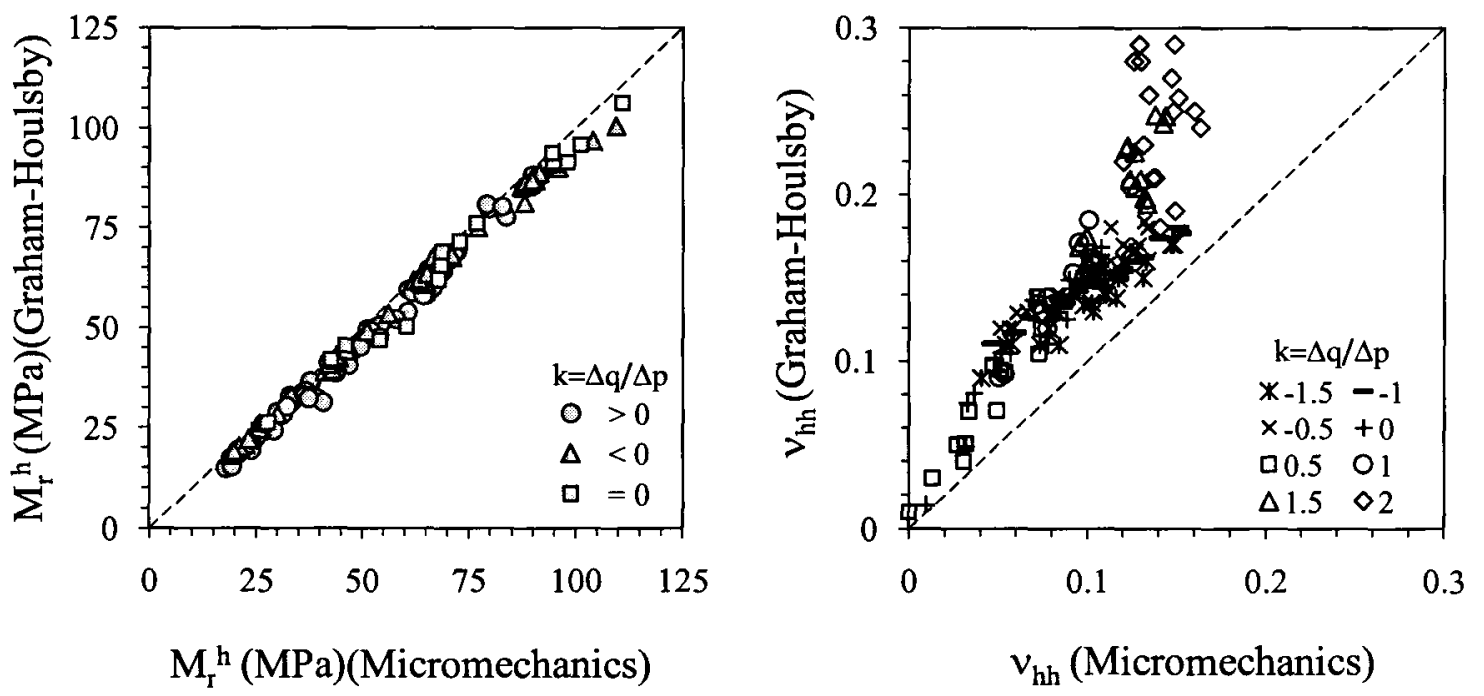

Fig. $6.6 v_{h h}$ and $M_{r}^{h}$ obtained from micromechanics model and the G-H assumption

For the stress path tests performed in this study, the horizontal resilient shear modulus $G_{r}^{h h}$ can be obtained from Eq. (4.15). After manipulating Eqs. (4.15), (6.17) and (6.19), the vertical resilient shear modulus $G_{r}^{v h}$ can be determined once $a$ and $k_{s} / k_{n}$ are known; i.e., 


$$
G_{r}^{v h}=\frac{(5-a)^{2}(14+2 a+r(21+12 a))}{(10+a)\left(-15 a^{2}+18 a+105+r(10+a)(7+a)\right)} M_{r}^{v}
$$

\subsection{FABRIC ANISOTROPY}

\subsubsection{Variation of $a$ and $k_{s} / k_{n}:$ Stress paths and $K_{\text {ini }}$}

It should be noted that there are some differences between very small strain behaviour and resilient behaviour of granular materials. For granular materials having very small strains, stress-induced change in $a$ and $k_{s} / k_{n}$ are very small and can be neglected. Consequently, the inherent/initial anisotropy dominates the very small strain behaviour of the material. On the other hand, owing to the amplitude of repeated stresses or strains, the resilient behaviour of a granular material is highly nonlinear, $a$ and $k_{s} / k_{n}$ of the material may change with the applied load. When a material's resilient strains recover during the unloading process, it's fabric may return to the initial state immediately before the load is applied, provided that the plastic deformation in each cycle of load application is negligible. For this reason, the variations of $a$ and $k_{s} / k_{n}$ along different stress paths are addressed in the following sections, as well as the effect of $K_{\text {ini }}$ on the development of $a$ and $k_{S} / k_{n}$.

\section{(a) Isotropic initial stress state}

The degree of fabric anisotropy $a$ and $k_{s} / k_{n}$ were back-calculated from the results of all stress path tests of test Series A and B using the micromechanics approach. Figure (6.7) shows that the variations of induced fabric anisotropy and $k_{s} / k_{n}$ for different stress paths under conditions of isotropic consolidation. It can be seen that the stiffness anisotropy 
primarily depends on the fabric anisotropy, with $M_{r}^{h} / M_{r}^{v}$ decreasing as $a$ increases.

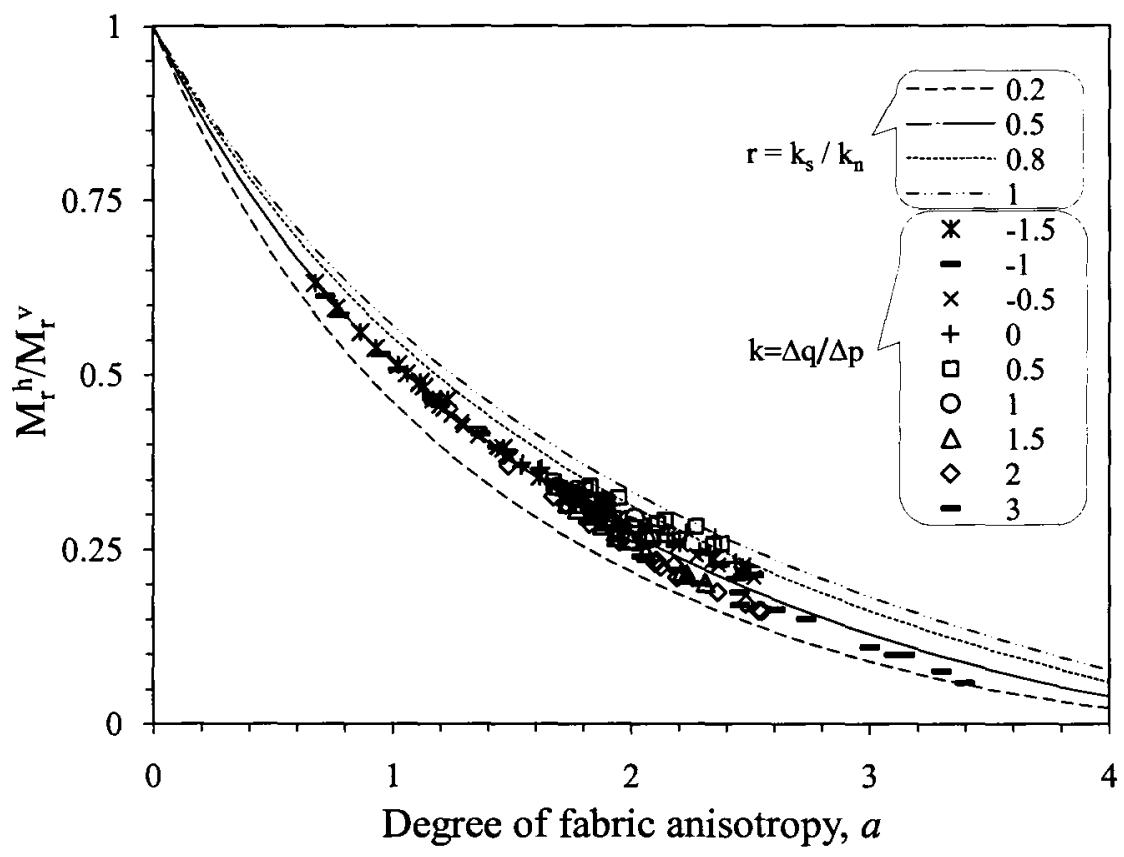

Fig. 6.7 Stiffness anisotropy and fabric anisotropy

Figure (6.8) shows that the resilient Poisson's ratios are however found to primarily depend on $k_{s} / k_{n}$, with the Poisson's ratios decreasing as $k_{s} / k_{n}$ increases in general. For stress paths having higher ratios of $\sigma_{v} / \sigma_{h}$ or $\Delta \sigma_{v} / \Delta \sigma_{h}$, such as stress paths with $k=$ 1.0 3.0, the lateral confinement becomes smaller than the vertical confinement, as a result, $k_{s} / k_{n}$ tends to decrease along the loading path, with $k_{s} / k_{n}$ for $k=2.0$ and 3.0 being smallest. Since $\sigma_{v}$ increases more rapidly than $\sigma_{h}$ along the loading path, the contact normals tend to concentrate in the vertical direction, thus the material becomes more anisotropic, both in the fabric and stiffness, with $a$ and $M_{r}^{h} / M_{r}^{v}$ for $k=3.0$ increasing and decreasing most rapidly, respectively. For stress paths with $k=0 \sim 0.5$, the variations in $\sigma_{v} / \sigma_{h}$ are small, the material is densely compacted in both the vertical 
and horizontal directions, which leads to an increase in $k_{s} / k_{n}$. As the particles cannot be significantly rearranged, although the stiffness and fabric anisotropy increase, the variation is relatively small.
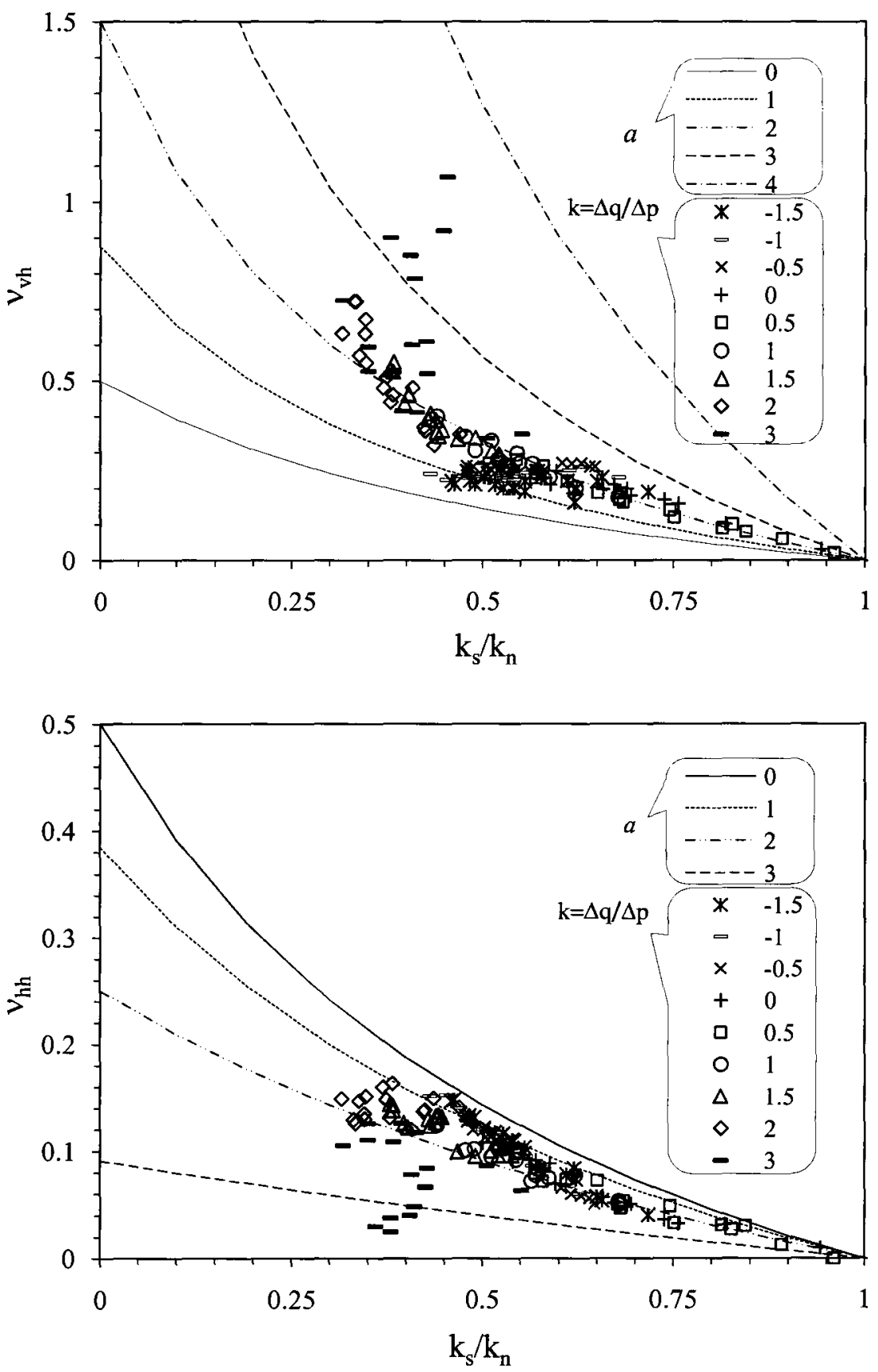

Fig. 6.8 Poisson's ratios and ratios of $k_{s} / k_{n}$ 
For stress paths with $k<0, \sigma_{v}<\sigma_{h}$; i.e., the major principal stress is in the horizontal direction, which is parallel to the horizontal bedding plane. The particles must therefore rearrange themselves to accommodate the stress changes, and the contact normal tends to concentrate in the horizontal direction, which leads to an increase in the deformation resistance in the horizontal direction when compared to the vertical direction. Consequently, both the stiffness and fabric anisotropy decrease, while $k_{s} / k_{n}$ increases.

\section{(b) Anisotropic initial stress state}

Figure 6.9 shows the dependency of $a$ and $k_{s} / k_{n}$ on $K_{i n i}$ for stress paths having $k=3.0$, respectively. It can be seen that $M_{r}^{h} / M_{r}^{v}$ and $a$ are well correlated, with the stiffness anisotropy consistently increasing with $a$ along the loading path. The relation of $\frac{M_{r}^{h}}{M_{r}^{v}} v S$ a is shown to be independent of $K_{\text {ini }}$. Recalling in Chapter 5, at a given lateral confinement with various $K_{i n i}$ values and when the final stress states are the same, $M_{r}^{h}$ and $M_{r}^{h}$ increases, and $v_{h h}$ and $v_{v h}$ decreases as the $K_{i n i}$ decreases, see Figs. 5.4 and 5.15. The induced stiffness anisotropy is however found to be identical and depend on the initial lateral confinement and subsequent $\sigma_{v} / \sigma_{h}$ ratio, regardless the values of $K_{i n i}$, see Figs. 5.12(b), (c) and 5.13. Therefore, the findings suggest that the fabric anisotropy, similar to stiffness anisotropy, depends on the initial and final stress states, which needs further examination.

In contrast to the variation of the stiffness anisotropy with $a$, the stiffness anisotropy is found to increase as $k_{s} / k_{n}$ decreases along the loading path, which is consistent with the common understanding. However, for various $K_{i n i}$, identical stiffness anisotropy and 
fabric anisotropy do not guarantee identical $k_{s} / k_{n}$. In other words, the induced $k_{s} / k_{n}$ depends on $K_{\text {ini }}$. Specifically, at a given initial lateral stress, when final stress states are the same, a lower ratio of $k_{s} / k_{n}$ is induced for a higher $K_{\text {ini }}$. These findings are most likely due to the effect of deviator stress on the deformation response of the material; i.e., larger deformations in both the horizontal and vertical directions are induced for a higher $K_{i n i}$, which in turn leads to a lower $k_{s} / k_{n}$.
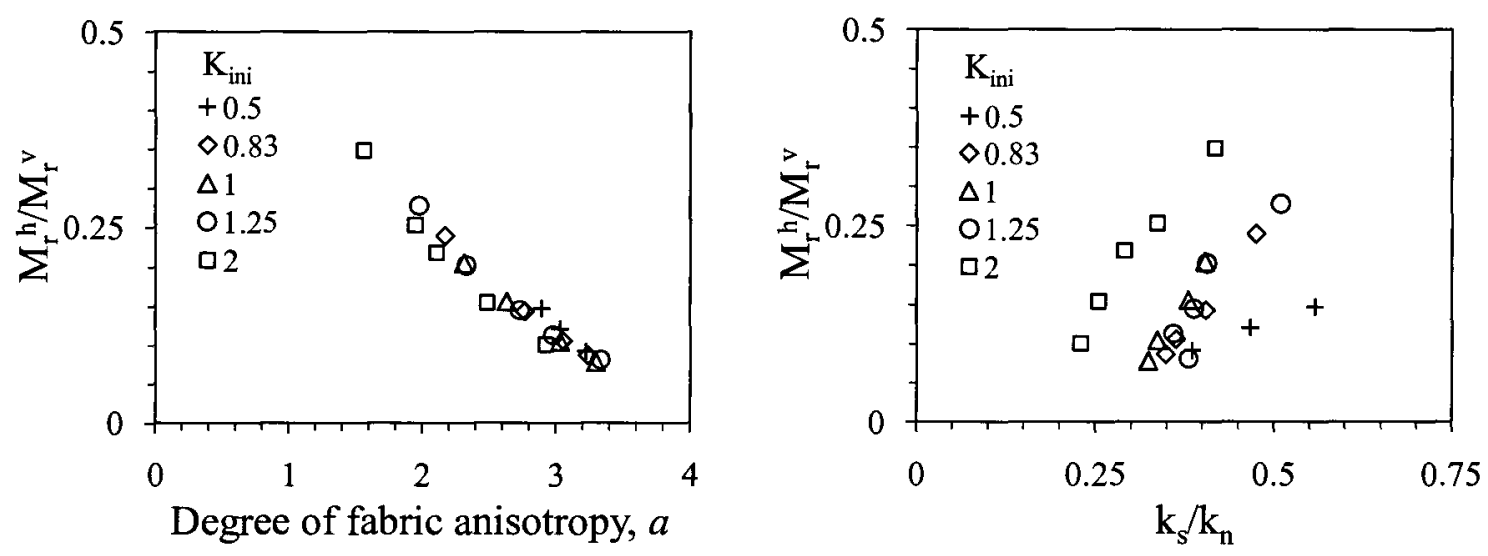

Fig. 6.9 Influence of $K_{i n i}$ on fabric anisotropy and $k_{s} / k_{n}$ for $k=3.0$

$$
\sigma_{h}^{i}=50 \mathrm{kPa}
$$

For stress paths with $k=-1.5$, as shown in Figs. 5.12(b), (d) and 5.13, at a given lateral confinement with various $K_{i n i}$ values, when $\sigma_{v} / \sigma_{h}$ at the final stress states are the same, the induced stiffness anisotropy appears to be identical and depend on the initial lateral confinement and subsequent $\sigma_{v} / \sigma_{h}$ ratio, regardless the values of $K_{i n i}$. The stiffness anisotropy and fabric anisotropy are also well correlated and independent of $K_{\text {ini }}$, with the induced stiffness anisotropy however decreasing as the fabric anisotropy decreases along the loading path; see Fig. 6.10. As the major principal stress is in the 
horizontal direction, the induced $k_{s} / k_{n}$ ratios are generally larger than those for $k=3.0$ and are insensitive to $K_{\text {ini }}$.
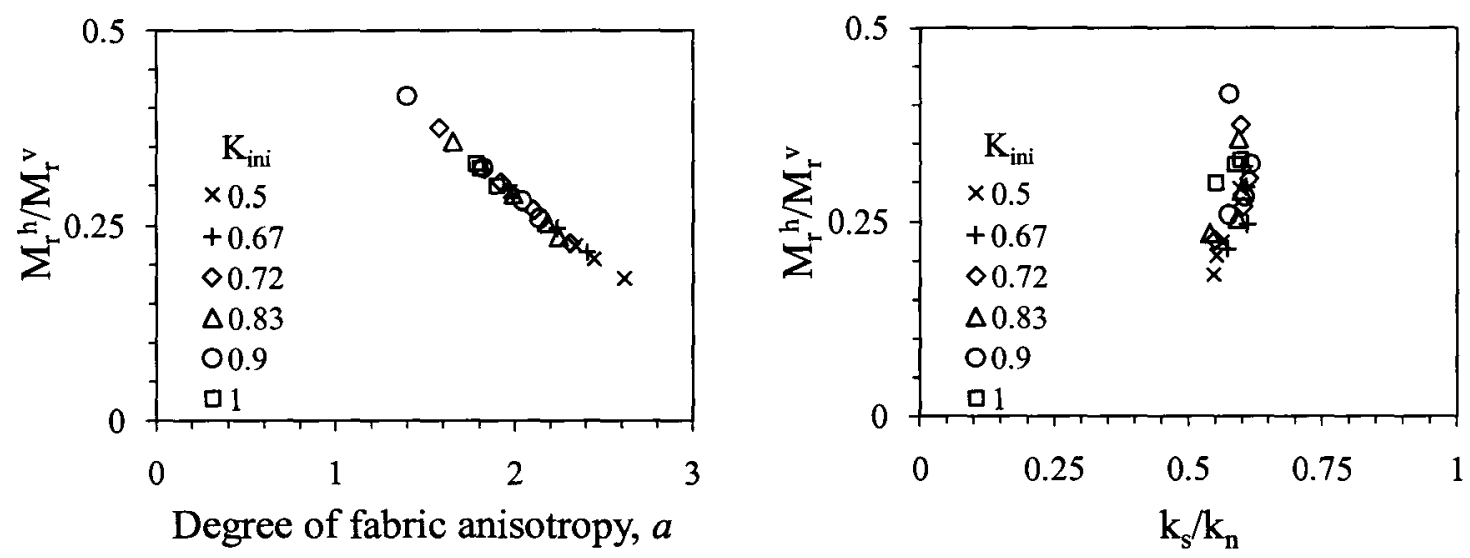

Fig. 6.10 Influence of $K_{\text {ini }}$ on fabric anisotropy and $k_{s} / k_{n}$ for $k=-1.5$

$$
\sigma_{h}^{i}=50 \mathrm{kPa}
$$

The good correlation between the stiffness anisotropy and fabric anisotropy, and their independence with respective to $K_{i n i}$ for stress paths having $k=-1.5$, also imply that the induced fabric anisotropy is influenced by the initial lateral confinement and the subsequent $\sigma_{v} / \sigma_{h}$, which is discussed in next section.

\subsubsection{Evolution of fabric anisotropy: fabric anisotropy and stress}

To account for the fabric anisotropy in constitutive models with embedded microstructure, a complete description of fabric evolution is necessary. In this research, the stress approach is used to describe the fabric evolution. The relation between the degree of fabric anisotropy and the initial stress state and subsequent stress history, including the stress path and final stress states, is investigated. 
Experiential evidence (Oda, 1985) shows that when a granular specimen is deformed, changes in the orientation of particles are related to the applied stresses. During a deformation process, contact normals tend to reorient themselves in the direction of major principal stress so as to maintain the resistance to external forces. As a result, the principal direction of fabric has a tendency to follow that of the major principal stress, and the components in the principal stress directions change at the same time.

\section{(a) Fabric evolution: isotropic initial stress state}

The degree of fabric anisotropy $a$ or the component of fabric tensor in the vertical direction $F_{1}$, is found to depend on the initial confinement and subsequent $\sigma_{v} / \sigma_{h}$ ratio. Figure 6.11(a) shows that the variations of $F_{1}$ along various stress paths for an isotropic initial stress state. One observes that $F_{1}$ tends to increase with an increase in $\sigma_{v} / \sigma_{h}$, which is consistent with the general understanding that contact normal tends to concentrate in the direction of the major principal stress to provide maximum resistance to deformation. Scrutinizing the experimental data reveals that the variation of $F_{1}$ with $\sigma_{v} / \sigma_{h}$ changes slightly along different stress paths, which is likely due to the effect of the mean effective stress on fabric evolution.

Figure 6.11(b) presents the evolution of $F_{1}$ with $\sigma_{v} / \sigma_{h}$ at different initial confining pressures along three representative stress paths where $k=-1.5,0.5$, and 3.0, respectively. As shown, an increase in the initial confinement tends to suppress fabric anisotropy for the same $\sigma_{v} / \sigma_{h}$. 


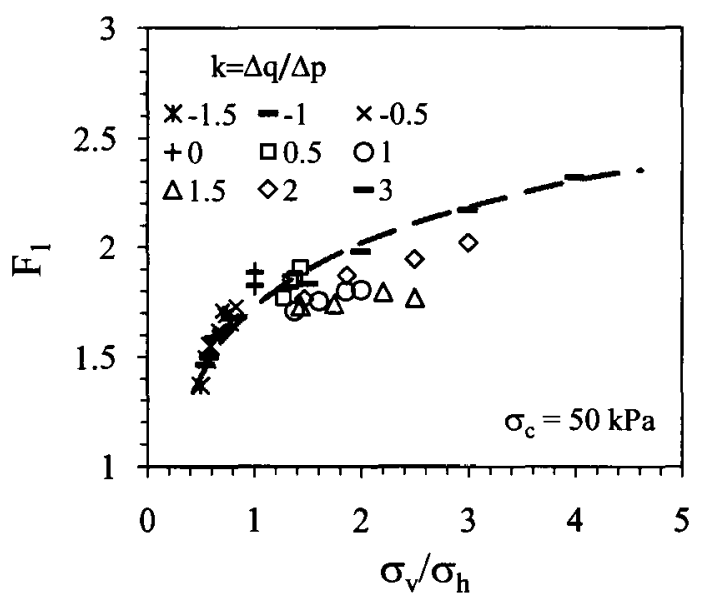

(a)

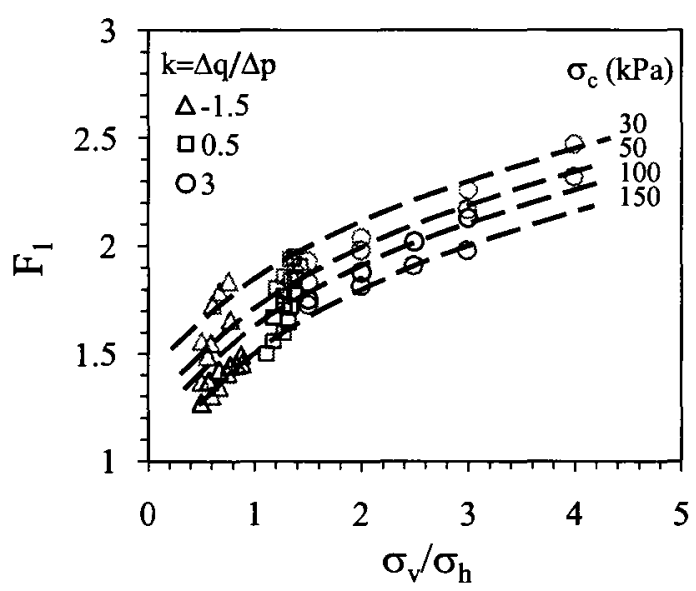

(b)

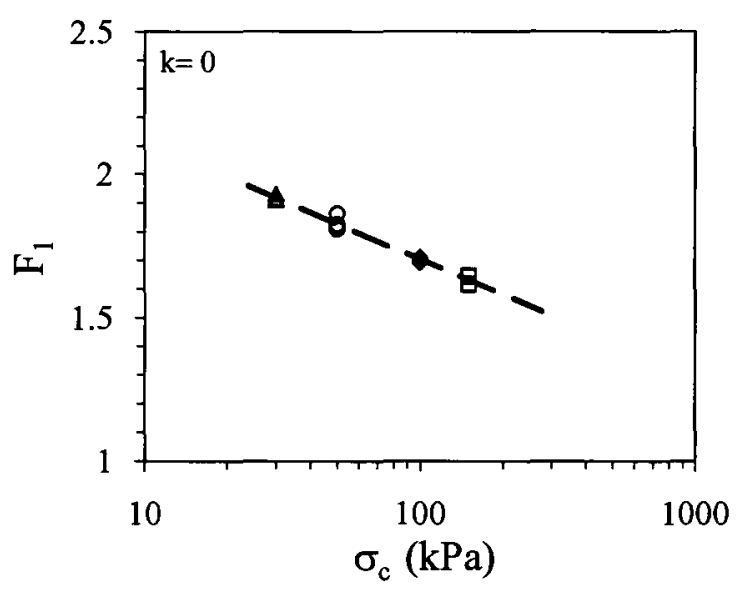

(c)

Fig. 6.11 Influence of stress on fabric: initial isotropic stress state 
The importance of confinement on fabric can also be observed from Fig. 6.11(c), which presents the variations of $F_{1}$ for the IC tests, where $\sigma_{v} / \sigma_{h}=1$. It can be seen that $F_{1}$ greatly depends on the initial confining pressures, the fabric change is very small along the loading path and can be considered as constant. This finding is consistent with the results in Chapter 4, which show that the $\varepsilon_{h, r} / \varepsilon_{v, r}$ ratio almost remains constant given $\sigma_{c}$, implying that the fabric change is very small, and can be neglected.

Therefore, the evolution of $F_{1}$ for different initial confining pressures along various stress paths can be quantitatively expressed as a power function of the initial lateral confinement $\left(\sigma_{c}=\sigma_{h}^{i}\right)$ and $\sigma_{v} / \sigma_{h}$ ratio, via

$$
F_{1}=g_{1}\left(\sigma_{c}\right)^{g_{2}}\left(\frac{\sigma_{v}}{\sigma_{h}}\right)^{g_{3}}
$$

where $g_{1}, g_{2}$ and $g_{3}$ are regression coefficients, with $g_{1}>0$ reflecting the material anisotropy, $g_{2}<0$ and $g_{3}>0$.

Figure 6.12 shows the curve fitting results for test Series $\mathrm{A}$. The figure demonstrates that the proposed Eq. (6.23) gives a reasonable fit to $F_{1}$ obtained from micromechanics approach and is capable of approximating the fabric anisotropy of a granular material at an isotropic/anisotropic stress state after undergoing a stress/strain history from an isotropic initial stress state.

As described previously, the variation of $k_{s} / k_{n}$ ratio depends on stress path. Unfortunately, the formulation of $k_{s} / k_{n}$ as a function of the applied stresses has not been obtained from the current test results and needs further investigation. 


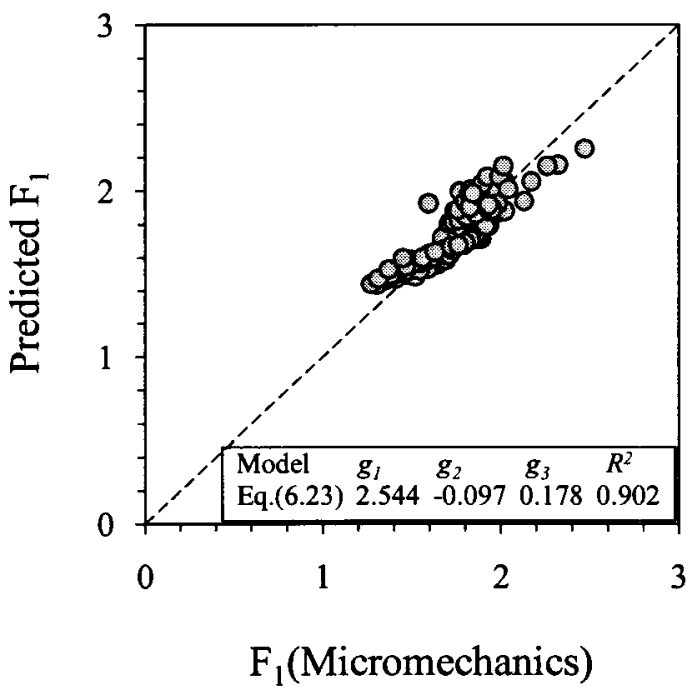

Fig. 6.12 Comparison of $F_{1}$ between micromechanics model and proposed approach

\section{(b) Fabric evolution: anisotropic initial stress state}

Figure 6.13 shows that the variation of $F_{1}$ along stress paths having $k=3.0$ and -1.5 for various $K_{\text {ini }}$ conditions. As expected, $F_{1}$ tends to increase with increased $\sigma_{v} / \sigma_{h}$ and is independent of $K_{i n i}$. It can be seen that the relation of $F_{1} v s \frac{\sigma_{v}}{\sigma_{h}}$ obtained from test Series B with various $K_{i n i}$ almost coincides with that from test Series A with $K_{i n i}=1$, which suggests that the stress-induced fabric anisotropy, similar to the stress-induced stiffness anisotropy, depends on the initial lateral confinement and subsequent $\sigma_{v} / \sigma_{h}$ ratio, regardless of the stress path and $K_{\text {ini }}$. Therefore, Eq. (6.23) can be used to estimate $F_{1}$ for conditions of both isotropic and anisotropic initial stress states by replacing the term $\sigma_{c}$ with $\sigma_{h}^{i}$ and becomes

$$
F_{1}=g_{1}\left(\sigma_{h}^{i}\right)^{g_{2}}\left(\frac{\sigma_{v}}{\sigma_{h}}\right)^{g_{3}}
$$




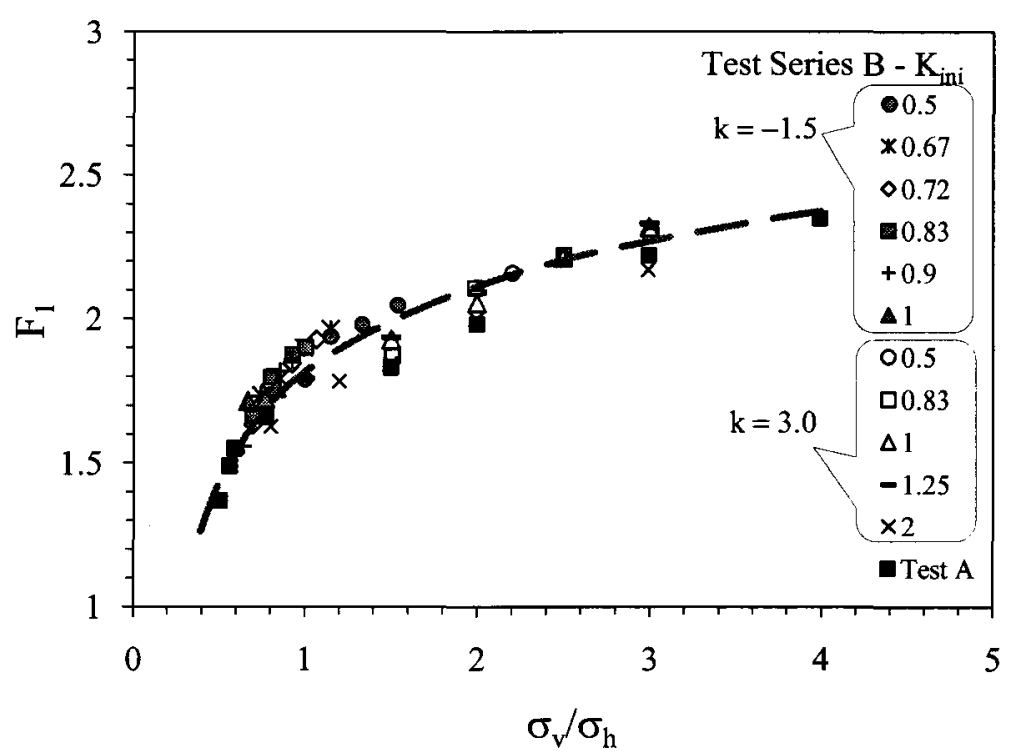

Fig. 6.13 Influence of stress on fabric: isotropic/anisotropic initial stress state $\sigma_{h}^{i}=50 \mathrm{kPa}$

\section{(c) Inherent/initial fabric anisotropy}

Similar to the derivation of the inherent/initial stiffness anisotropy in previous chapters, the initial/inherent fabric anisotropy for an anisotropic/isotropic initial stress state can be obtained from Eqs. (6.23) and (6.24). The inherent and initial fabric anisotropy are given by

$$
\begin{gathered}
\left(F_{1}\right)_{\text {inherent }}=g_{1}\left(\sigma_{c}\right)^{g_{2}} \\
\left(F_{1}\right)_{\text {initial }}=g_{1}\left(\sigma_{h}^{i}\right)^{g_{2}}\left(K_{\text {ini }}\right)^{-g_{3}}
\end{gathered}
$$

Equations (6.24) and (6.26) suggest that the initial fabric anisotropy is influenced by $K_{i n i}$, while the induced fabric anisotropy is independent of $K_{i n i}$. The proposed evolution law of fabric anisotropy works well for the prediction of $F_{1}$. Further calibration is necessary through extensive tests for various $K_{i n i}$ conditions and initial stress states along 
various stress paths.

\subsection{SUMMARY}

This chapter interpreted the tests results for both isotropic and anisotropic initial stress states along various stress paths with regard to fabric anisotropy. A micromechanics model was established to back-calculate the degree of fabric anisotropy $a$ and $k_{s} / k_{n}$ ratio. It is shown that the micromechanics model can be used to predict the resilient properties, such as $v_{h h}, M_{r}^{h}$ and $G_{r}^{v h}$.

Scrutinizing the experimental data, a relation was proposed to approximate the induced fabric anisotropy for both isotropic and anisotropic initial stress state. The induced fabric anisotropy of the test material is shown to depend on the initial lateral confinement and subsequent $\sigma_{v} / \sigma_{h}$ ratio at the final stress state, and be independent of $K_{\text {ini }}$. It increases with an increase in $\sigma_{v} / \sigma_{h}$, but decreases with the initial lateral confinement. The initial fabric anisotropy is however found to decrease as the initial confinement and $K_{\text {ini }}$ increases.

With respect to $k_{s} / k_{n}$, it is found to depend on stress path and $K_{i n i}$. For stress paths with $k=3.0, k_{s} / k_{n}$ decreases with an increase in $K_{\text {ini }}$. For stress paths with $k=-1.5$, $k_{s} / k_{n}$ is generally larger than those with $k=3.0$ and insensitive to $K_{i n i}$. 


\section{CONCLUSION AND FUTURE WORK}

The research reported in this thesis focused on the investigation of the cross-anisotropic resilient properties of coarse grained soil. An important factor considered was the influence of stress path on the resilient modulus and Poisson's ratio, in both the vertical and horizontal directions from an isotropic/anisotropic initial stress state. Relatively simple, but comprehensive resilient modulus and Poisson's ratio equations were developed, which can be used to predict the resilient modulus and Poisson's ratio subjected to general stress conditions.

The following observations and conclusions are made based on the research accomplished in this study:

- Different deformation characteristics were observed for the stress path tests performed in the study. The possible combinations of deformation in general are: contractive in both the vertical and horizontal directions; contractive in the vertical (horizontal) direction and extensive in the horizontal (vertical) direction; and contractive in the vertical (horizontal) direction and very small horizontal (vertical) strain. The different patterns of stress-strain curves are attributed to both the anisotropic nature of the material and the applied stresses, no matter if the stresses are anisotropic or isotropic. Owing to the various deformation characteristics, the variations of resilient properties with stress changes depend on stress path. In general, the vertical resilient modulus is higher than the horizontal resilient modulus, which is attributed to disposition or compaction process. 
- The inherent stiffness and fabric anisotropy of the test material is found to depend on initial confining pressure. When the confining pressure increases, the material tends to become less anisotropic. The stiffness and fabric anisotropy from IC tests is almost constant for a given initial confining pressure. For stress paths with positive $k$, the stiffness anisotropy increases as stresses become more anisotropic. However, when the major principal stresses are in the horizontal direction, the stiffness anisotropy decreases.

- The resilient response of a granular material is influenced by $K_{i n i}$. Tests results have shown the resilient properties are influenced by $K_{i n i}$, In particular, $M_{r}^{v}$ is more influenced by $K_{i n i}$ than $M_{r}^{h}$.

- Test results show that the common preferred resilient modulus equations, such as the Uzan and AASHTO equations are not capable of characterizing the crossanisotropic resilient modulus subjected to general stress paths. In addition to the initial lateral confinement, the cross-anisotropic resilient modulus is found to depend on the resilient deformation developed during the stress path test and/or $K_{\text {ini }}$. For stress paths with $k \neq 0$, the vertical resilient modulus can be expressed as a function of initial lateral confinement, $K_{i n i}$ and the resilient strain in the direction of minor principal stress. And the horizontal resilient modulus can be approximated as a function of initial lateral confinement and the horizontal resilient strain.

- Both the induced stiffness anisotropy and fabric anisotropy are independent of $K_{i n i}$. Test results have shown that the induced stiffness anisotropy and fabric 
anisotropy increase with an increase in $\sigma_{v} / \sigma_{h}$ ratio, but decreases as the initial confining pressure increases. However, the initial fabric anisotropy and stiffness anisotropy depend on $K_{\text {ini }}$.

The following work is considered necessary in the future:

- Further experiments on the tested material for the purpose of understanding the anisotropic resilient properties subjected to the major principal stress rotation.

- Further calibration of the proposed resilient modulus equations and fabric evolution law through more extensive stress path tests.

- Extension of resilient modulus equation for general stress states, including the influence of stress path, major principal stress rotation and $K_{i n i}$.

- Implementation of the resilient modulus equations into a finite element program for modelling the responses and predictions of the performance of flexible pavement. 


\section{APPENDIX I PROGRAM FOR TESTS}

Table AI. 1 Program for test Series A

\begin{tabular}{|c|c|c|c|c|c|}
\hline \multirow{2}{*}{ Test Code } & \multirow{2}{*}{$\begin{array}{c}\text { Slope of } \\
\text { stress path }\end{array}$} & \multirow{2}{*}{$\begin{array}{l}\text { Initial stress ( } \mathrm{kPa} \text { ) } \\
\sigma_{c}=\sigma_{h}=\sigma_{v}\end{array}$} & \multicolumn{3}{|c|}{ Cyclic stress ( $k P a)$} \\
\hline & & & $\Delta \sigma_{h}$ & $\Delta \sigma_{v}$ & $\Delta q$ \\
\hline \multirow{16}{*}{ A_CTC } & \multirow{16}{*}{3.0} & \multirow{4}{*}{30} & 0 & 15 & 15 \\
\hline & & & 0 & 30 & 30 \\
\hline & & & 0 & 60 & 60 \\
\hline & & & 0 & 90 & 90 \\
\hline & & \multirow{4}{*}{50} & 0 & 15 & 15 \\
\hline & & & 0 & 25 & 25 \\
\hline & & & 0 & 35 & 35 \\
\hline & & & 0 & 50 & 50 \\
\hline & & \multirow{4}{*}{100} & 0 & 50 & 50 \\
\hline & & & 0 & 100 & 100 \\
\hline & & & 0 & 150 & 150 \\
\hline & & & 0 & 200 & 200 \\
\hline & & \multirow{4}{*}{150} & 0 & 75 & 75 \\
\hline & & & 0 & 150 & 150 \\
\hline & & & 0 & 225 & 225 \\
\hline & & & 0 & 300 & 300 \\
\hline \multirow{16}{*}{ A_PL4 } & \multirow{16}{*}{2.0} & \multirow{4}{*}{30} & 3 & 21 & 18 \\
\hline & & & 5 & 35 & 30 \\
\hline & & & 10 & 70 & 60 \\
\hline & & & 15 & 105 & 90 \\
\hline & & \multirow{4}{*}{50} & 4 & 29 & 25 \\
\hline & & & 8 & 58 & 50 \\
\hline & & & 17 & 117 & 100 \\
\hline & & & 25 & 175 & 150 \\
\hline & & \multirow{4}{*}{100} & 8 & 58 & 50 \\
\hline & & & 17 & 117 & 100 \\
\hline & & & 25 & 175 & 150 \\
\hline & & & 33 & 233 & 200 \\
\hline & & \multirow{4}{*}{150} & 13 & 88 & 75 \\
\hline & & & 25 & 175 & 150 \\
\hline & & & 38 & 263 & 225 \\
\hline & & & 50 & 350 & 300 \\
\hline \multirow{16}{*}{ A_PL3 } & \multirow{16}{*}{1.5} & \multirow{4}{*}{30} & 5 & 20 & 15 \\
\hline & & & 10 & 40 & 30 \\
\hline & & & 20 & 80 & 60 \\
\hline & & & 30 & 120 & 90 \\
\hline & & \multirow{4}{*}{50} & 8 & 33 & 25 \\
\hline & & & 17 & 67 & 50 \\
\hline & & & 33 & 133 & 100 \\
\hline & & & 50 & 200 & 150 \\
\hline & & \multirow{4}{*}{100} & 17 & 67 & 50 \\
\hline & & & 33 & 133 & 100 \\
\hline & & & 50 & 200 & 150 \\
\hline & & & 67 & 267 & 200 \\
\hline & & & 25 & 100 & 75 \\
\hline & & 150 & 50 & 200 & 150 \\
\hline & & 130 & 75 & 300 & 225 \\
\hline & & & 100 & 400 & 300 \\
\hline
\end{tabular}


Table AI.1 Program for test Series A (continued)

\begin{tabular}{|c|c|c|c|c|c|}
\hline \multirow{2}{*}{ Test Code } & \multirow{2}{*}{$\begin{array}{c}\text { Slope of } \\
\text { stress path }\end{array}$} & \multirow{2}{*}{$\begin{array}{c}\text { Initial stress ( } \mathrm{kPa} \text { ) } \\
\sigma_{c}=\sigma_{h}=\sigma_{v}\end{array}$} & \multicolumn{3}{|c|}{ Cyclic stress $(\mathrm{kPa})$} \\
\hline & & & $\Delta \sigma_{h}$ & $\Delta \sigma_{v}$ & $\Delta q$ \\
\hline \multirow{16}{*}{ A_PL2 } & \multirow{16}{*}{1.0} & \multirow[t]{4}{*}{ 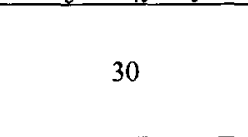 } & 10 & 25 & 15 \\
\hline & & & 20 & 50 & 30 \\
\hline & & & 40 & 100 & 60 \\
\hline & & & 60 & 150 & 90 \\
\hline & & \multirow{4}{*}{50} & 17 & 42 & 25 \\
\hline & & & 33 & 83 & 50 \\
\hline & & & 67 & 167 & 100 \\
\hline & & & 100 & 250 & 150 \\
\hline & & \multirow{4}{*}{100} & 33 & 83 & 50 \\
\hline & & & 67 & 167 & 100 \\
\hline & & & 100 & 250 & 150 \\
\hline & & & 133 & 333 & 200 \\
\hline & & \multirow{4}{*}{150} & 50 & 125 & 75 \\
\hline & & & 100 & 250 & 150 \\
\hline & & & 150 & 375 & 225 \\
\hline & & & 200 & 500 & 300 \\
\hline \multirow{17}{*}{ A_PL1 } & \multirow{17}{*}{0.5} & \multirow{4}{*}{30} & 15 & 24 & 9 \\
\hline & & & 25 & 40 & 15 \\
\hline & & & 38 & 60 & 22 \\
\hline & & & 50 & 80 & 30 \\
\hline & & \multirow{5}{*}{50} & 20 & 32 & 12 \\
\hline & & & 42 & 67 & 25 \\
\hline & & & 63 & 100 & 38 \\
\hline & & & 83 & 133 & 50 \\
\hline & & & 125 & 200 & 75 \\
\hline & & \multirow{4}{*}{100} & 42 & 67 & 25 \\
\hline & & & 83 & 133 & 50 \\
\hline & & & 125 & 200 & 75 \\
\hline & & & 167 & 267 & 100 \\
\hline & & \multirow{4}{*}{150} & 33 & 53 & 20 \\
\hline & & & 63 & 100 & 38 \\
\hline & & & 125 & 200 & 75 \\
\hline & & & 167 & 267 & 100 \\
\hline \multirow{18}{*}{ A_IC } & \multirow{18}{*}{0} & \multirow{4}{*}{30} & 10 & 10 & 0 \\
\hline & & & 15 & 15 & 0 \\
\hline & & & 22 & 22 & 0 \\
\hline & & & 30 & 30 & 0 \\
\hline & & \multirow{5}{*}{50} & 15 & 15 & 0 \\
\hline & & & 25 & 25 & 0 \\
\hline & & & 35 & 35 & 0 \\
\hline & & & 50 & 50 & 0 \\
\hline & & & 75 & 75 & 0 \\
\hline & & & 15 & 15 & 0 \\
\hline & & & 30 & 30 & 0 \\
\hline & & 100 & 50 & 50 & 0 \\
\hline & & & 100 & 100 & 0 \\
\hline & & & 30 & 30 & 0 \\
\hline & & & 50 & 50 & 0 \\
\hline & & 150 & 75 & 75 & 0 \\
\hline & & & 100 & 100 & 0 \\
\hline & & & 150 & 150 & 0 \\
\hline
\end{tabular}


Table AI.1 Program for test Series A (continued)

\begin{tabular}{|c|c|c|c|c|c|}
\hline \multirow{2}{*}{ Test Code } & \multirow{2}{*}{$\begin{array}{c}\text { Slope of } \\
\text { stress path }\end{array}$} & \multirow{2}{*}{$\begin{array}{c}\text { Initial stress }(\mathrm{kPa}) \\
\sigma_{c}=\sigma_{h}=\sigma_{v}\end{array}$} & \multicolumn{3}{|c|}{ Cyclic stress $(k P a)$} \\
\hline & & & $\Delta \sigma_{h}$ & $\Delta \sigma_{v}$ & $\Delta q$ \\
\hline \multirow{20}{*}{ A_PL5 } & \multirow{20}{*}{-0.5} & \multirow[t]{5}{*}{ r } & 23 & 13 & -10 \\
\hline & & & 35 & 20 & -15 \\
\hline & & & 48 & 28 & -20 \\
\hline & & & 70 & 40 & -30 \\
\hline & & & 105 & 60 & -45 \\
\hline & & \multirow{5}{*}{50} & 35 & 20 & -15 \\
\hline & & & 58 & 33 & -25 \\
\hline & & & 88 & 50 & -38 \\
\hline & & & 117 & 67 & -50 \\
\hline & & & 175 & 100 & -75 \\
\hline & & \multirow{5}{*}{100} & 35 & 20 & -15 \\
\hline & & & 70 & 40 & -30 \\
\hline & & & 117 & 67 & -50 \\
\hline & & & 175 & 100 & -75 \\
\hline & & & 233 & 133 & -100 \\
\hline & & \multirow{5}{*}{150} & 47 & 27 & -20 \\
\hline & & & 70 & 40 & -30 \\
\hline & & & 117 & 67 & -50 \\
\hline & & & 175 & 100 & -75 \\
\hline & & & 233 & 133 & -100 \\
\hline \multirow{20}{*}{ A_PL6 } & \multirow{20}{*}{-1.0} & \multirow{5}{*}{30} & 12 & 3 & -9 \\
\hline & & & 20 & 5 & -15 \\
\hline & & & 28 & 7 & -21 \\
\hline & & & 40 & 10 & -30 \\
\hline & & & 60 & 15 & -45 \\
\hline & & \multirow{5}{*}{50} & 20 & 5 & -15 \\
\hline & & & 33 & 8 & -25 \\
\hline & & & 50 & 13 & -38 \\
\hline & & & 67 & 17 & -50 \\
\hline & & & 100 & 25 & -75 \\
\hline & & \multirow{5}{*}{100} & 20 & 5 & -15 \\
\hline & & & 40 & 10 & -30 \\
\hline & & & 67 & 17 & -50 \\
\hline & & & 100 & 25 & -75 \\
\hline & & & 133 & 33 & -100 \\
\hline & & \multirow{5}{*}{150} & 27 & 7 & -20 \\
\hline & & & 40 & 10 & -30 \\
\hline & & & 67 & 17 & -50 \\
\hline & & & 100 & 25 & -75 \\
\hline & & & 133 & 33 & -100 \\
\hline & & & 10 & 0 & -10 \\
\hline & & & 15 & 0 & -15 \\
\hline & & 30 & 20 & 0 & -20 \\
\hline & & & 30 & 0 & -30 \\
\hline & & & 45 & 0 & -45 \\
\hline & & & 15 & 0 & -15 \\
\hline & & & 35 & 0 & -35 \\
\hline & & 50 & 40 & 0 & -40 \\
\hline & & & 50 & 0 & -50 \\
\hline A_CTE & -1.5 & & 15 & 0 & -15 \\
\hline & & & 30 & 0 & -30 \\
\hline & & 100 & 50 & 0 & -50 \\
\hline & & & 75 & 0 & -75 \\
\hline & & & 100 & 0 & -100 \\
\hline & & & 20 & 0 & -20 \\
\hline & & & 30 & 0 & -30 \\
\hline & & 150 & 50 & 0 & -50 \\
\hline & & & 75 & 0 & -75 \\
\hline & & & 100 & 0 & -100 \\
\hline
\end{tabular}


Table AI. 2 Program for test Series B

\begin{tabular}{|c|c|c|c|c|c|c|}
\hline \multirow{2}{*}{ Test Code } & \multirow{2}{*}{$\begin{array}{c}\text { Slope of } \\
\text { stress path }\end{array}$} & \multirow{2}{*}{$K_{\text {ini }}$} & \multirow{2}{*}{$\begin{array}{c}\text { Initial stress }(\mathrm{kPa}) \\
\left(\sigma_{h}, \sigma_{v}\right)\end{array}$} & \multicolumn{3}{|c|}{ Cyclic stress ( $k P a)$} \\
\hline & & & & $\Delta \sigma_{h}$ & $\Delta \sigma_{v}$ & $\Delta q$ \\
\hline \multirow{21}{*}{ B_CTC } & \multirow{21}{*}{3.0} & \multirow{3}{*}{0.5} & \multirow{3}{*}{$(50,100)$} & 0 & 10 & 10 \\
\hline & & & & 0 & 25 & 25 \\
\hline & & & & 0 & 50 & 50 \\
\hline & & \multirow{4}{*}{0.83} & \multirow{4}{*}{$(50,60)$} & 0 & 15 & 15 \\
\hline & & & & 0 & 40 & 40 \\
\hline & & & & 0 & 65 & 65 \\
\hline & & & & 0 & 90 & 90 \\
\hline & & \multirow{4}{*}{1} & \multirow{4}{*}{$(50,50)$} & 0 & 25 & 25 \\
\hline & & & & 0 & 50 & 50 \\
\hline & & & & 0 & 75 & 75 \\
\hline & & & & 0 & 100 & 100 \\
\hline & & \multirow{5}{*}{1.25} & \multirow{5}{*}{$(50,40)$} & 0 & 10 & 10 \\
\hline & & & & 0 & 35 & 35 \\
\hline & & & & 0 & 60 & 60 \\
\hline & & & & 0 & 85 & 85 \\
\hline & & & & 0 & 110 & 110 \\
\hline & & \multirow{5}{*}{2} & \multirow{5}{*}{$(50,25)$} & 0 & 15 & 15 \\
\hline & & & & 0 & 35 & 35 \\
\hline & & & & 0 & 50 & 50 \\
\hline & & & & 0 & 75 & 75 \\
\hline & & & & 0 & 125 & 125 \\
\hline \multirow{23}{*}{ B_CTE } & \multirow{23}{*}{-1.5} & \multirow{3}{*}{1.0} & \multirow{3}{*}{$(50,50)$} & 10 & 0 & -10 \\
\hline & & & & 15 & 0 & -15 \\
\hline & & & & 25 & 0 & -25 \\
\hline & & \multirow{4}{*}{0.9} & \multirow{4}{*}{$(50,55)$} & 10 & 0 & -5 \\
\hline & & & & 15 & 0 & -10 \\
\hline & & & & 25 & 0 & -20 \\
\hline & & & & 37 & 0 & -32 \\
\hline & & & & 10 & 0 & 0 \\
\hline & & & & 15 & 0 & -5 \\
\hline & & 0.83 & $(50,60)$ & 25 & 0 & -15 \\
\hline & & & & 37 & 0 & -27 \\
\hline & & & & 15 & 0 & 5 \\
\hline & & & & 25 & 0 & -5 \\
\hline & & 0.72 & $(50,70)$ & 37 & 0 & -17 \\
\hline & & & & 50 & 0 & -30 \\
\hline & & & & 15 & 0 & 10 \\
\hline & & 067 & $(5075)$ & 25 & 0 & 0 \\
\hline & & $0.6 \%$ & $(50, / 5)$ & 37 & 0 & -12 \\
\hline & & & & 50 & 0 & -25 \\
\hline & & & & 15 & 0 & 35 \\
\hline & & 05 & $(50,100)$ & 25 & 0 & 25 \\
\hline & & 0.0 & $(50,100)$ & 37 & 0 & 13 \\
\hline & & & & 50 & 0 & 0 \\
\hline
\end{tabular}


Table AI. 3 Specifications of testing equipment

\begin{tabular}{l|c|c}
\hline Type of measurement & Full scale & Resolution \\
\hline Axial load & $\pm 10 \mathrm{kN}$ & $0.1 \mathrm{~N}$ \\
Torque & $\pm 300 \mathrm{~N} \cdot \mathrm{m}$ & $0.01 \mathrm{~N} \cdot \mathrm{m}$ \\
Volume change & $100 \mathrm{cc}$ & $0.1 \mathrm{cc}$ \\
Radial displacement & $\pm 50 \mathrm{~mm}$ & $1.5 \mathrm{Micron}$ \\
Axial displacement & $\pm 25 \mathrm{~mm}$ & $0.01 \mathrm{~mm}$ \\
Pore water pressure & $1000 \mathrm{kPa}$ & $0.1 \mathrm{kPa}$ \\
Inner cell pressure & $1000 \mathrm{kPa}$ & $0.1 \mathrm{kPa}$ \\
Outer cell pressure & $1000 \mathrm{kPa}$ & $0.1 \mathrm{kPa}$ \\
Inner cell volume change & $100 \mathrm{cc}$ & $0.1 \mathrm{cc}$ \\
Servo actuator frequency & up to $70 \mathrm{~Hz}$ & \\
Data acquisition speed & up to $5 \mathrm{kHz}$ & \\
Data acquisition & & $20 \mathrm{bit}$ \\
\hline
\end{tabular}
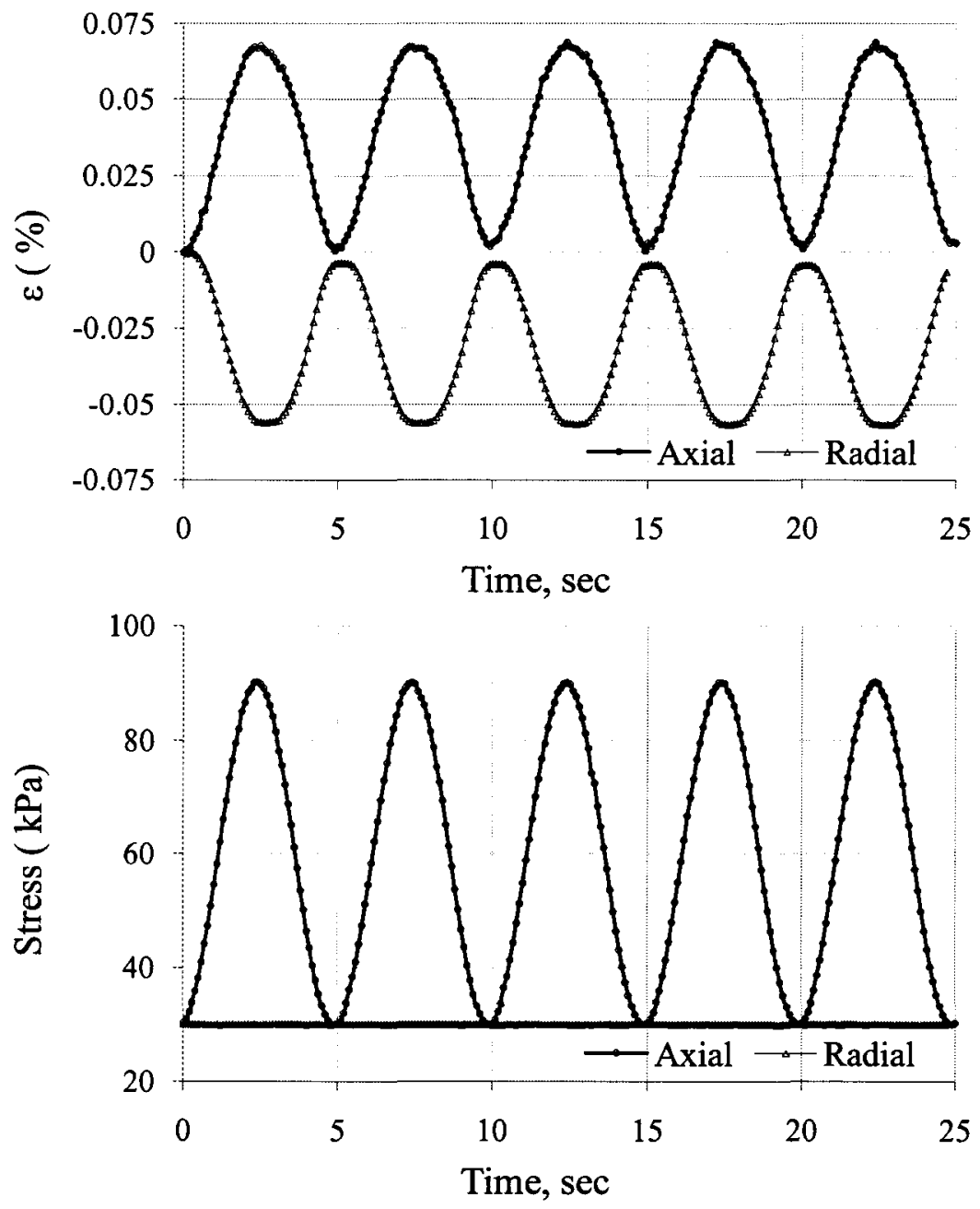

Fig. AI. 1 Sample of stresses and deformation measured in tests 


\section{APPENDIX II ADDITIONAL FIGURES}
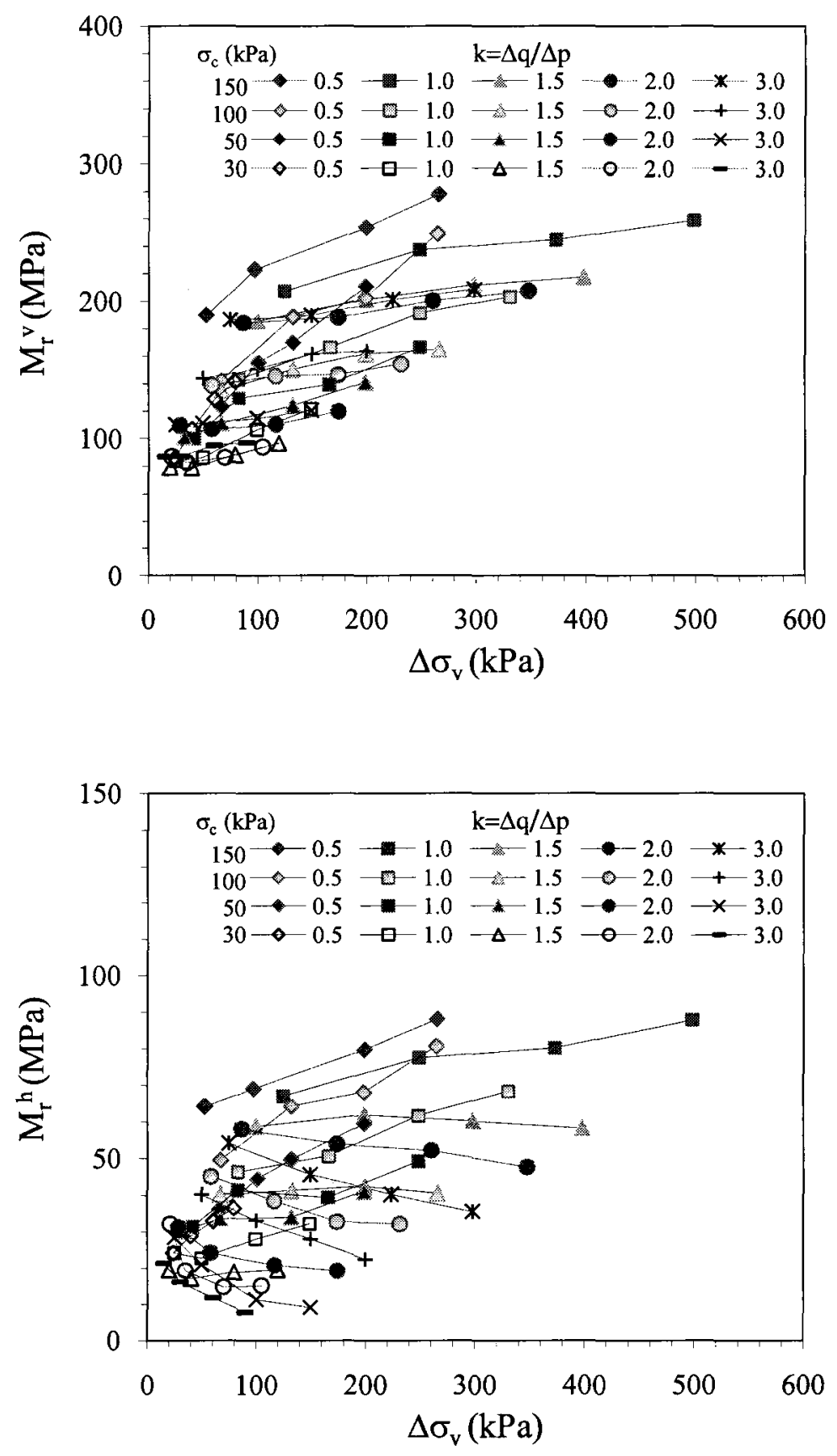

Fig. AII. 1 Resilient moduli and stress increments for stress paths $k>0$ 

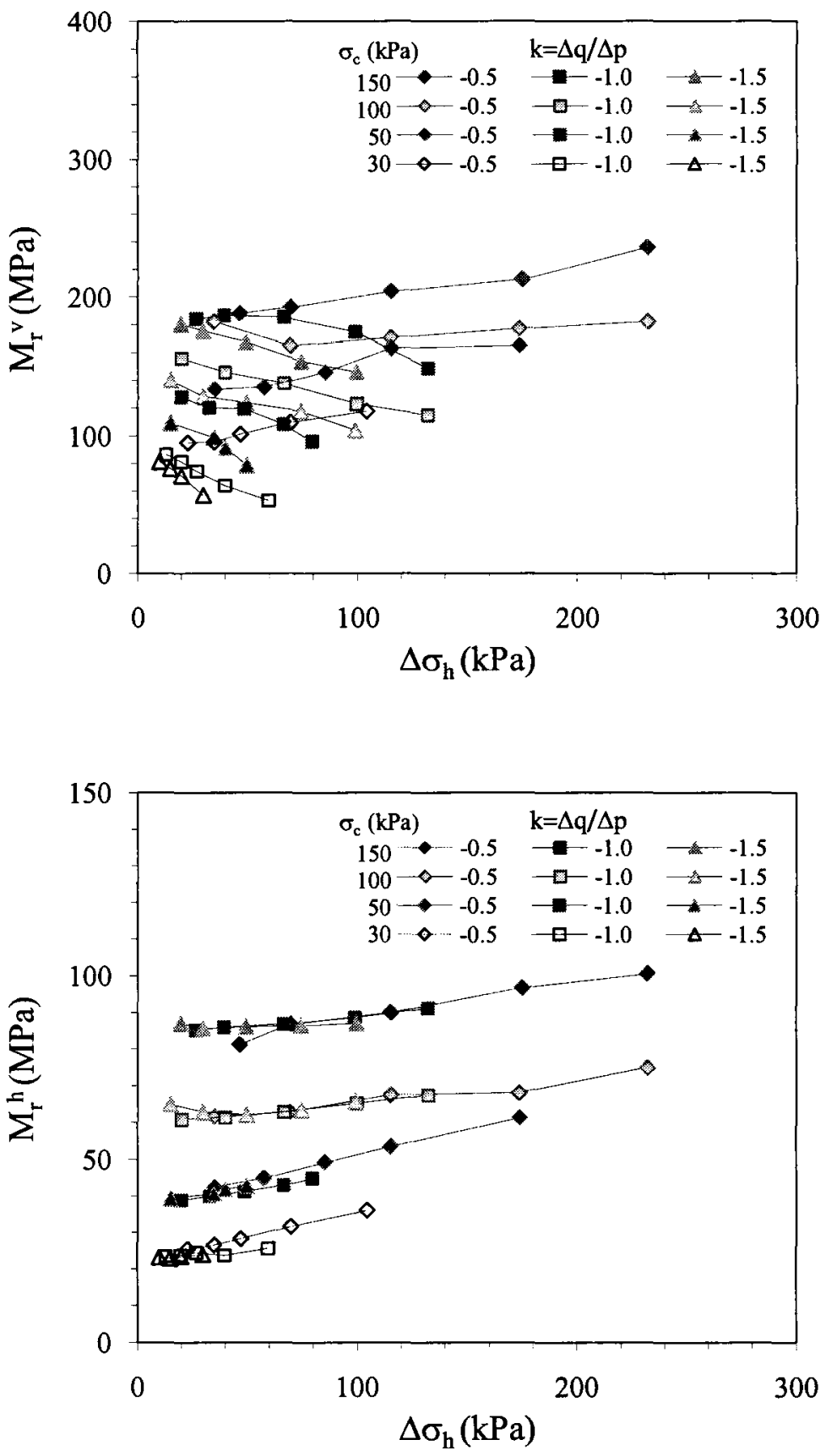

Fig. AII. 2 Resilient moduli and stress increments for stress paths $k<0$ 

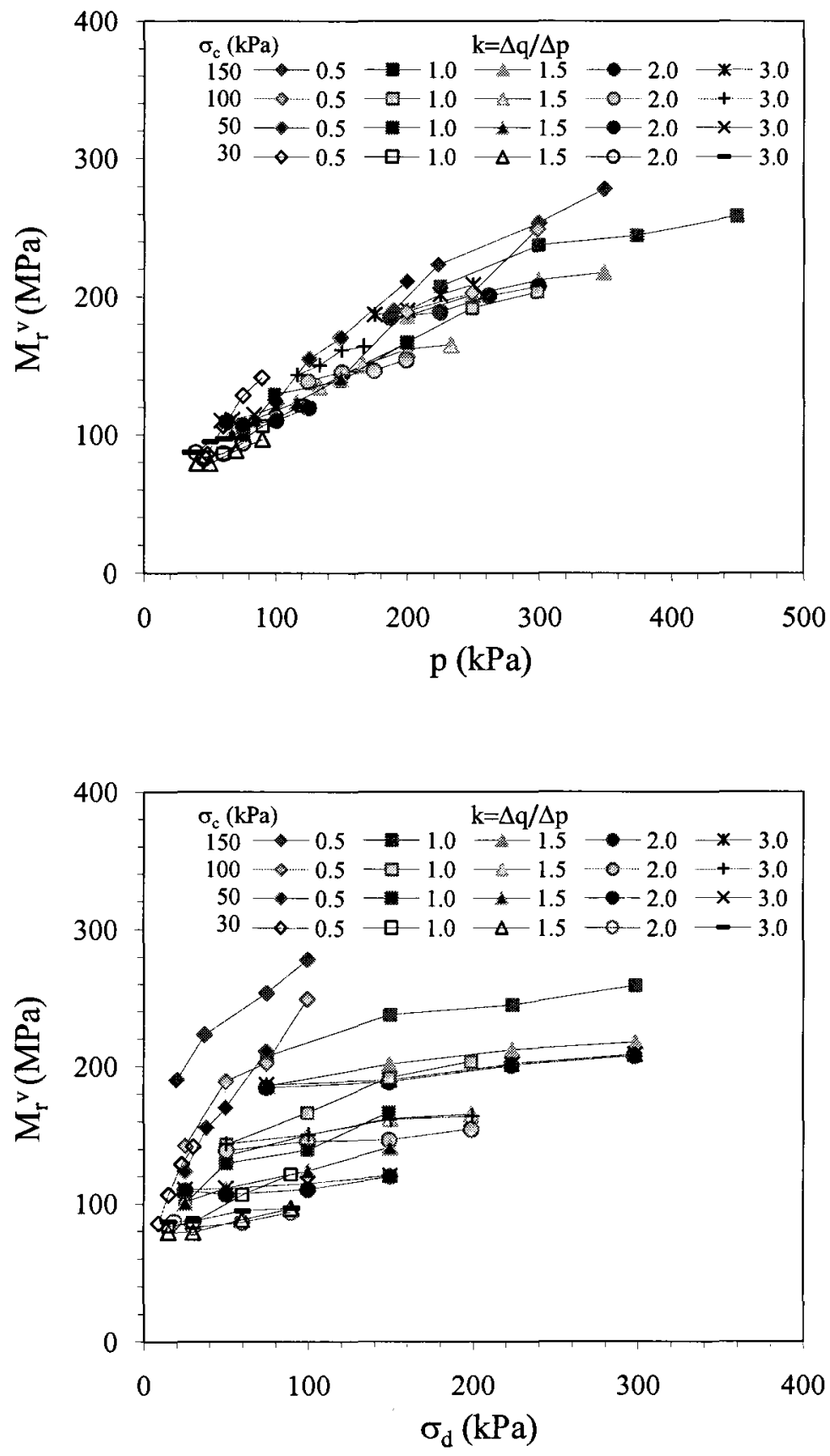

Fig. AII. 3 Vertical resilient moduli and stress increments for stress paths $k>0$ 

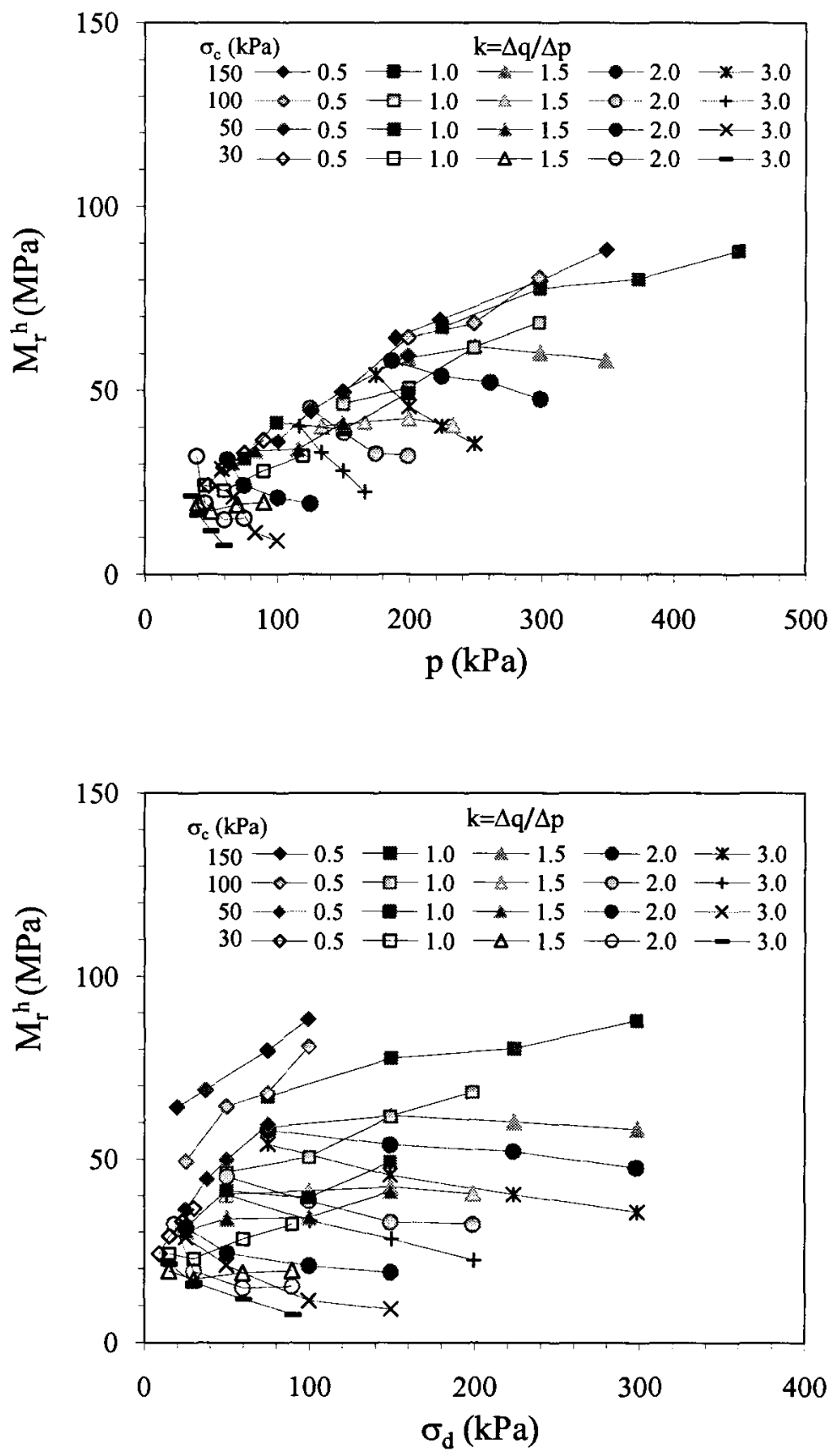

Fig. AII. 4 Horizontal resilient moduli and stress increments for stress paths $k>0$ 

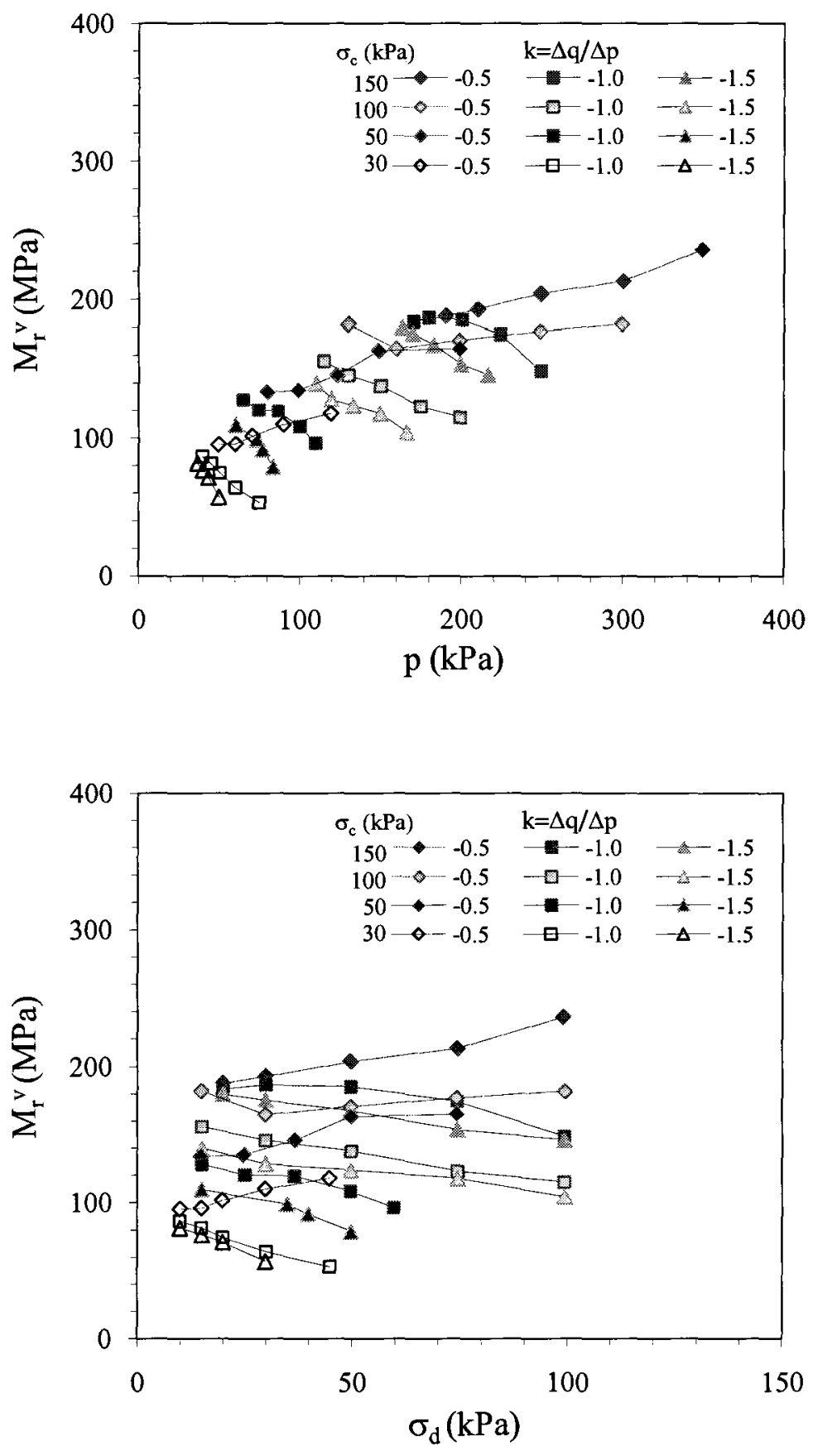

Fig. AII. 5 Vertical resilient moduli and stress increments for stress paths $k<0$ 

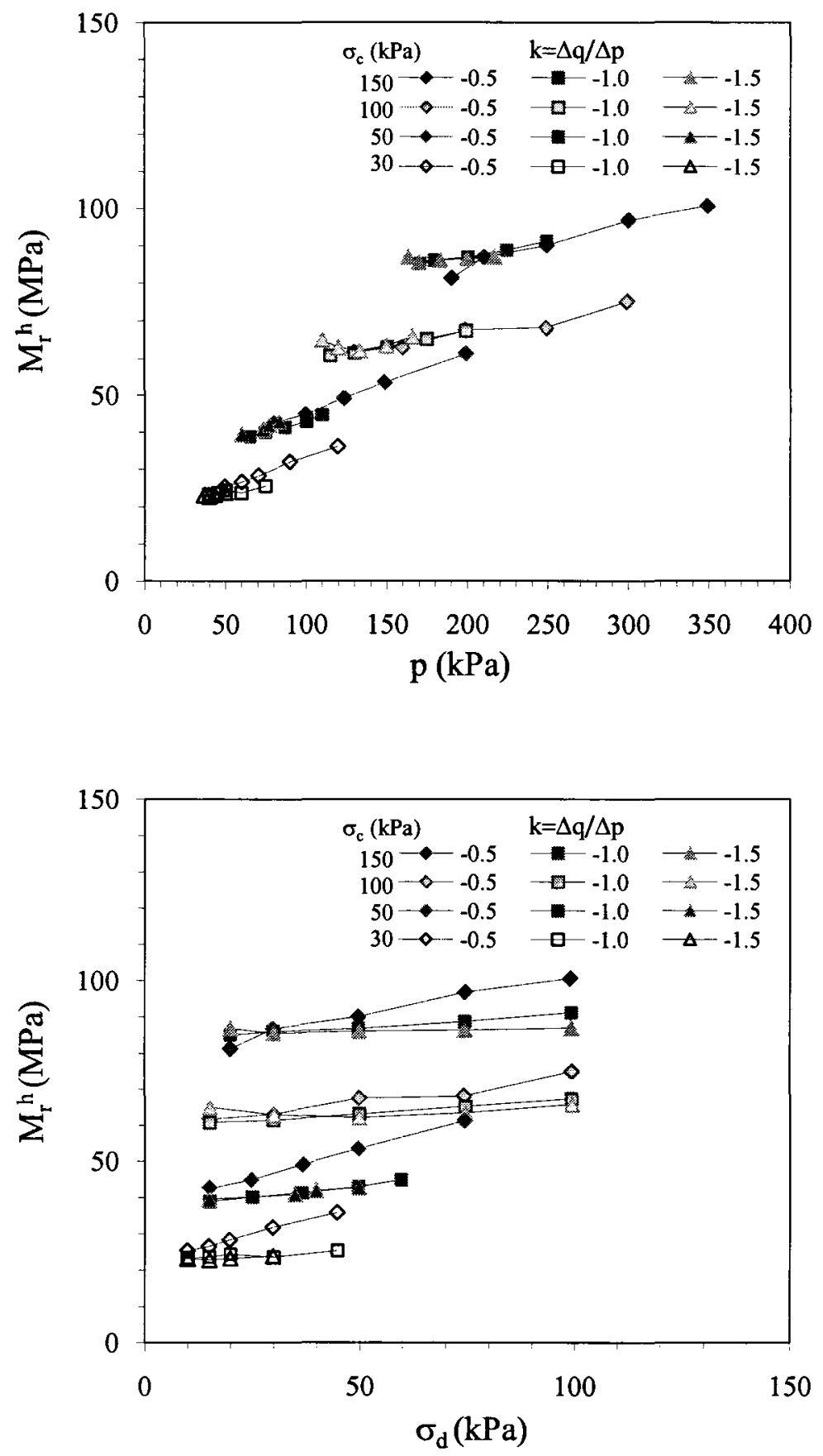

Fig. AII. 6 Horizontal resilient moduli and stress increments for stress paths $k<0$ 

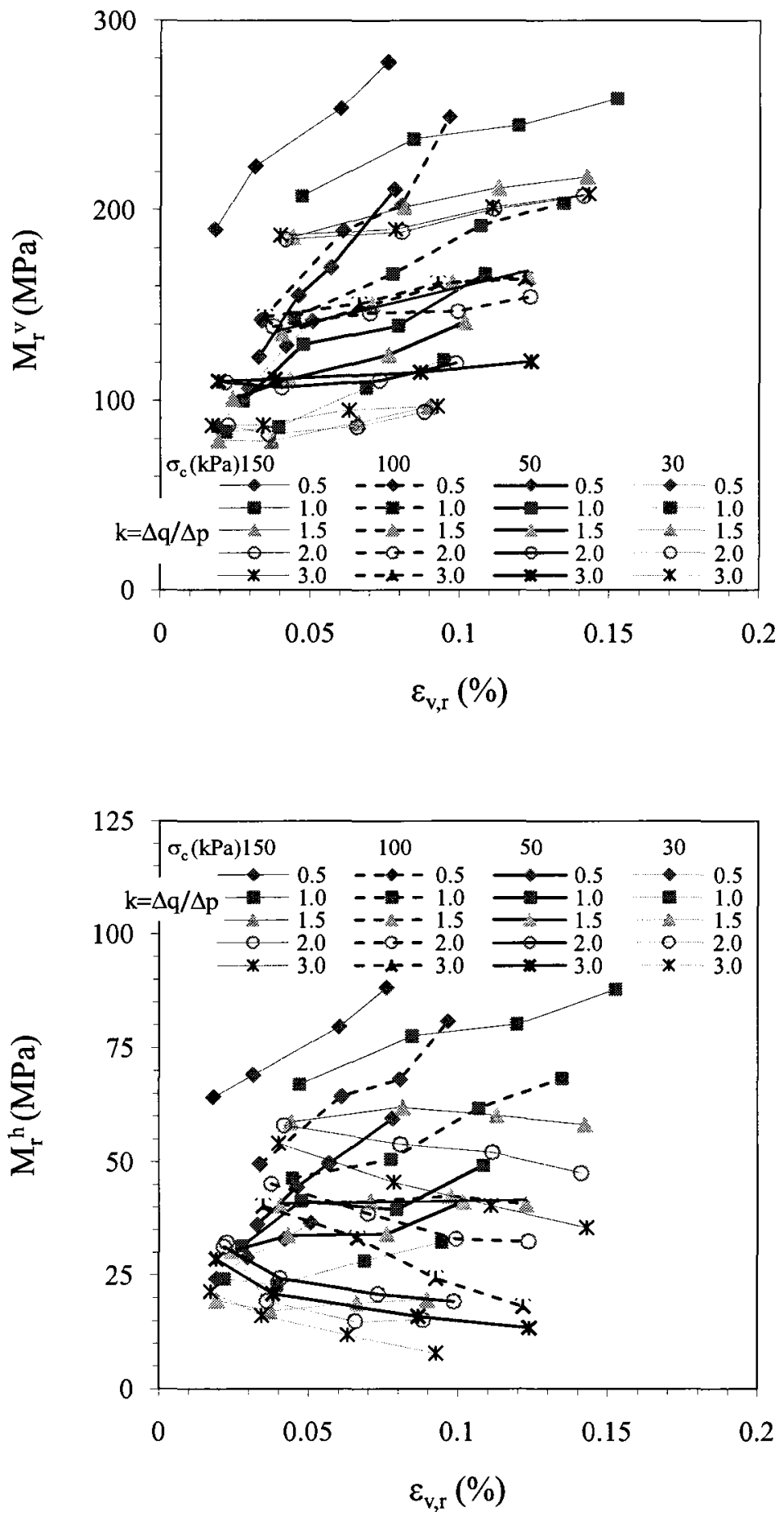

Fig. AII. 7 Resilient moduli and resilient strain components for stress paths $k>0$

Continued next page 

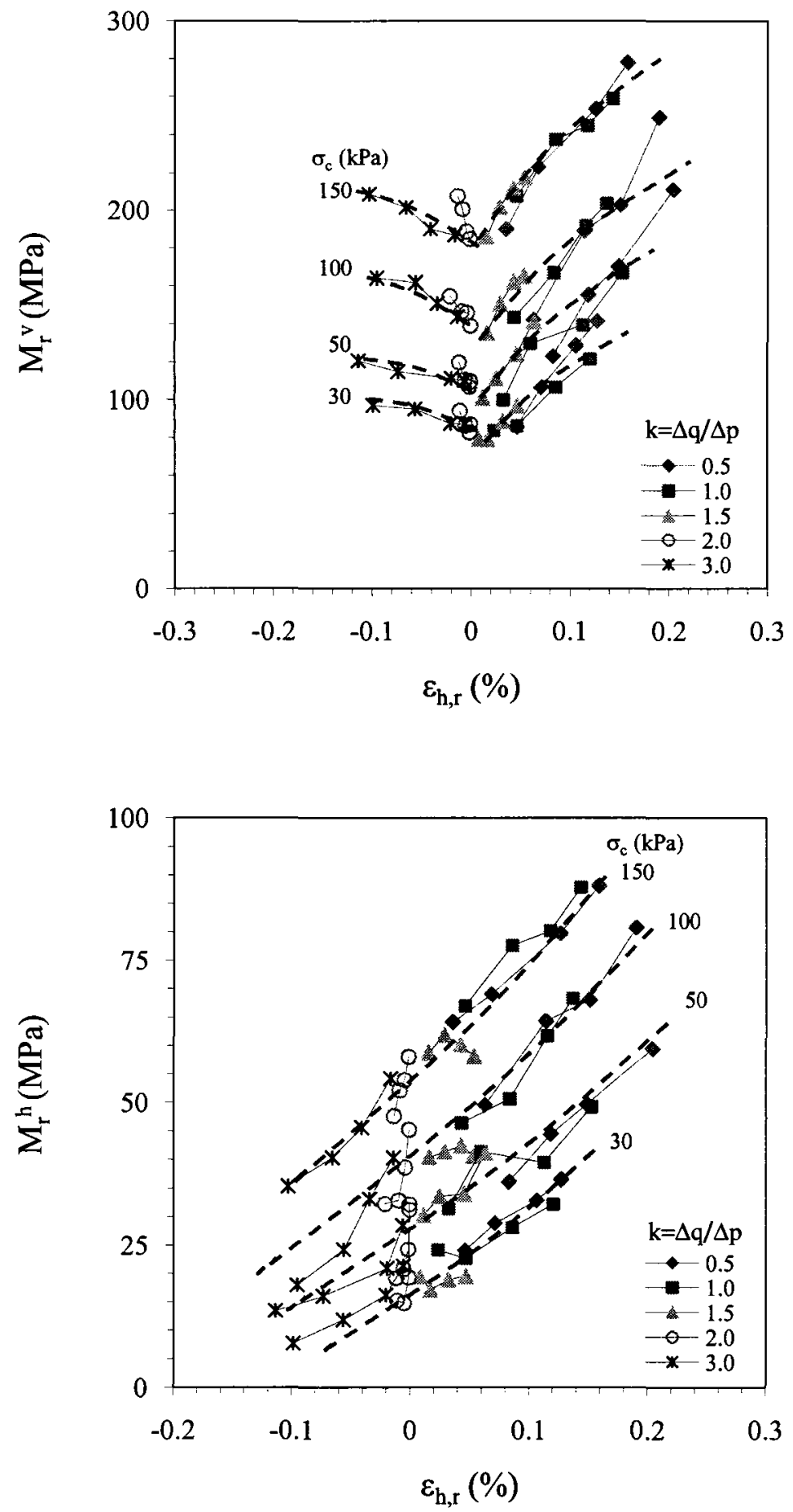

Fig. AII. 7 Resilient moduli and resilient strain components for stress paths $k>0$ 

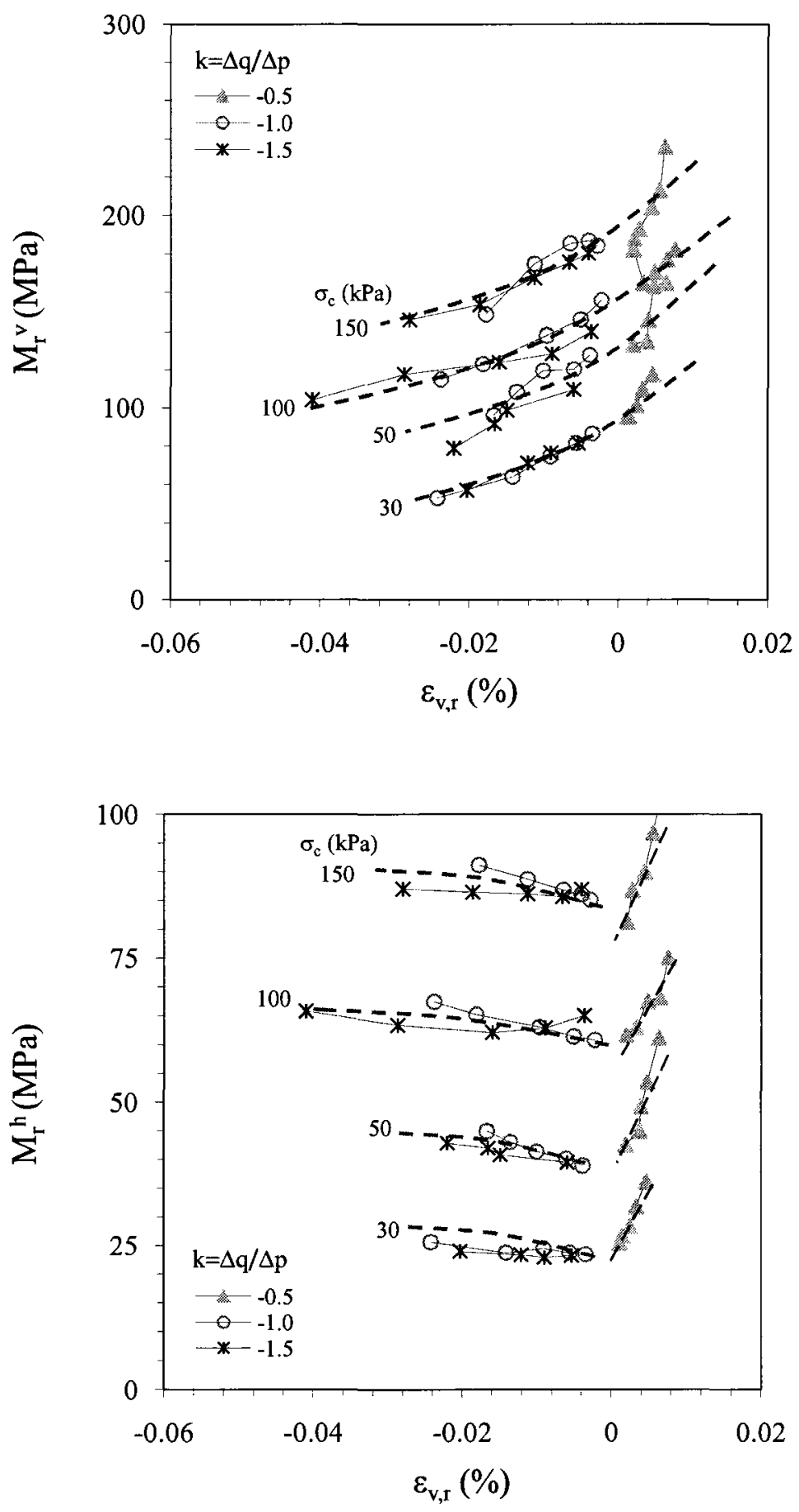

Fig. AII. 8 Resilient moduli and resilient strain components for stress paths $k<0$

Continued next page 

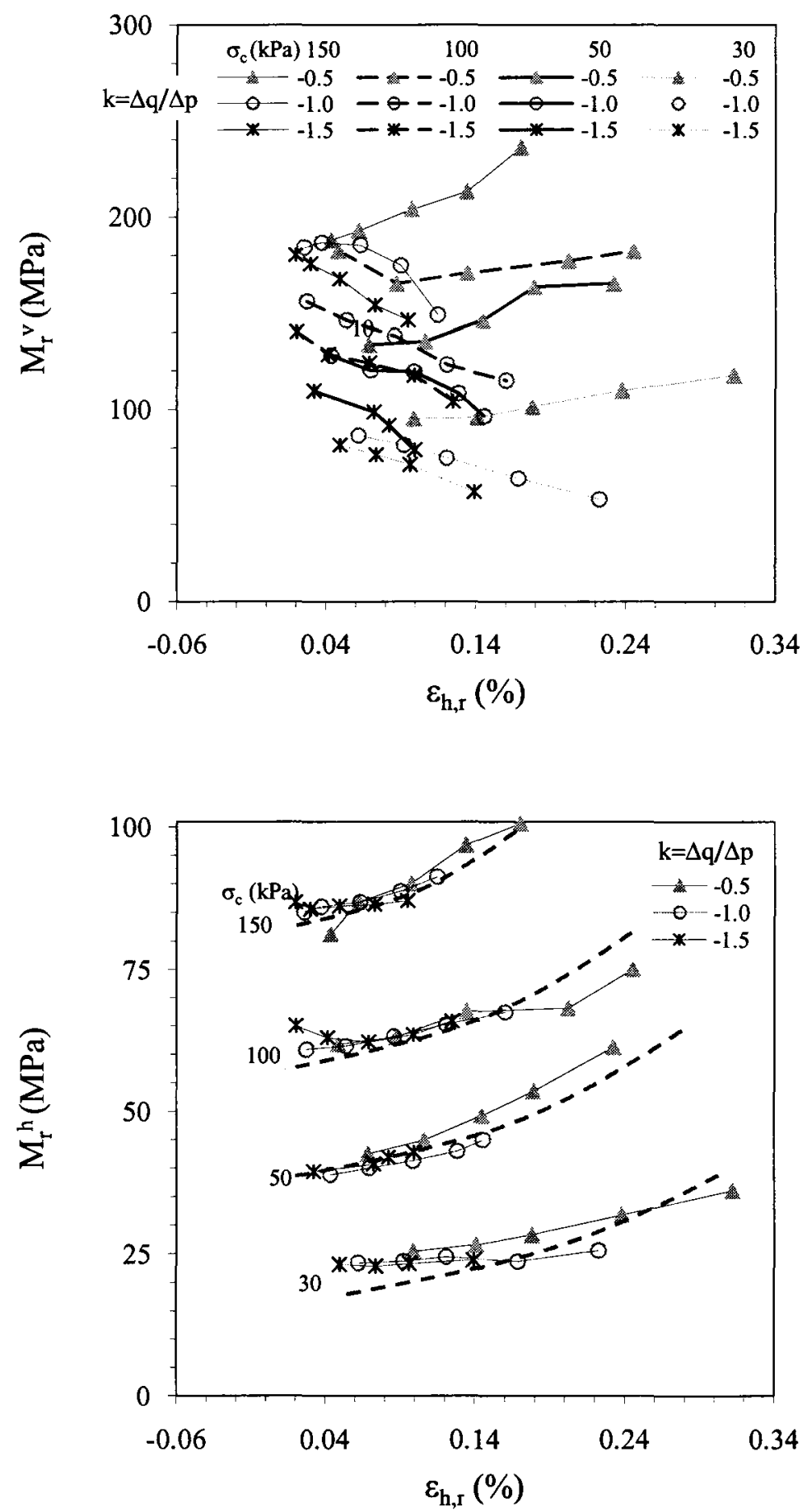

Fig. AII.8 Resilient moduli and resilient strain components for stress paths $k<0$ 

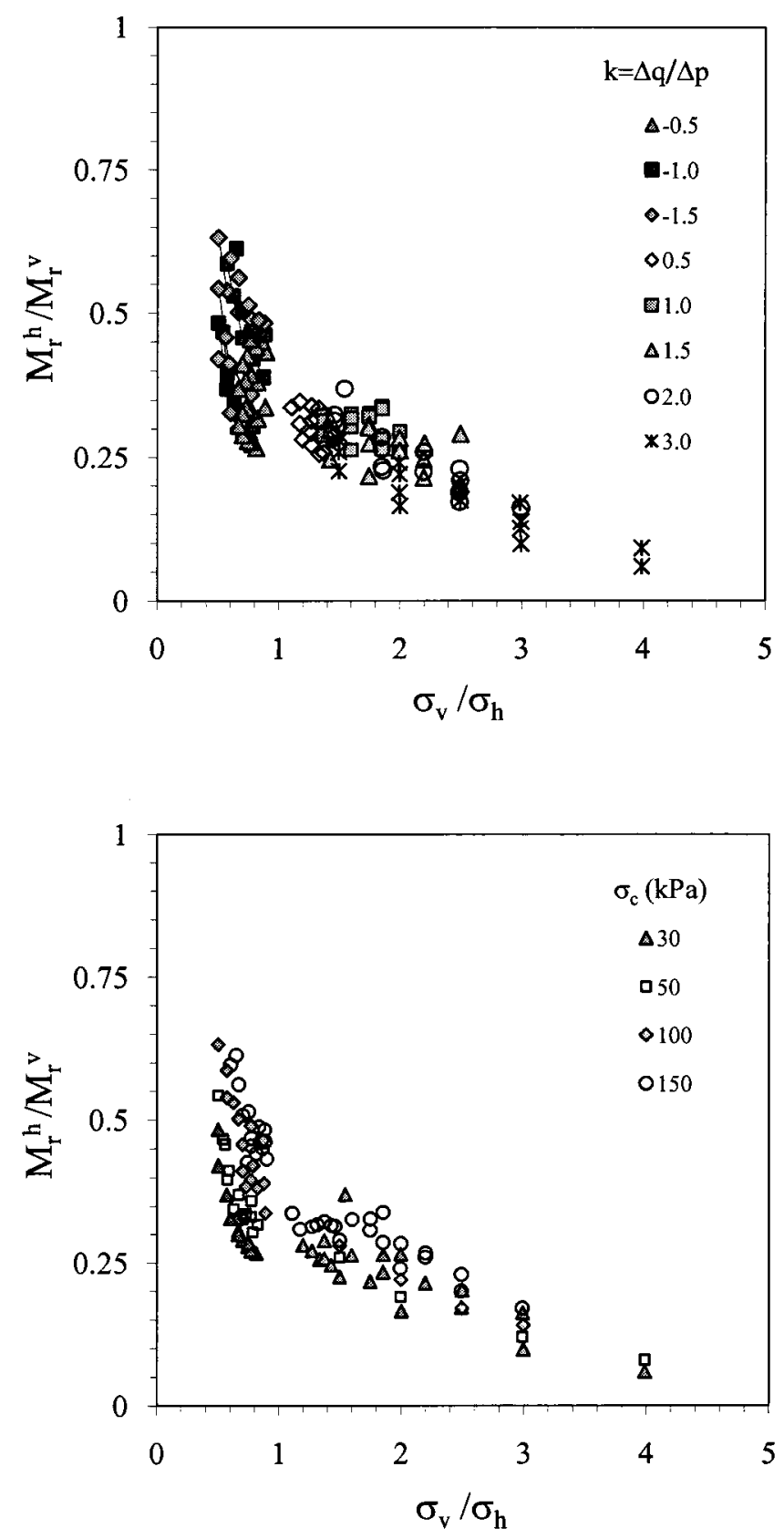

Fig. AII. 9 Stress-induced stiffness anisotropy 


\section{APPENDIX III}

\section{COMPONENTS OF A COMPLIANCE MATRIX}

As we know,

$$
\begin{aligned}
& E(\gamma, \beta)=\frac{1}{4 \pi}\left(1+\frac{a}{4}(3 \cos (2 \gamma+1))\right. \\
& F_{i j}=\frac{N}{V} \int_{0}^{2 \pi \pi} \int_{0}^{2} l_{i} l_{j} E(n) \sin \gamma d \gamma d \beta
\end{aligned}
$$

where $0 \leq \beta \leq 2 \pi$, and independent of $\gamma, 0 \leq \gamma \leq \pi, l_{i}=2 R n_{i}$ For cross-anisotropic fabric, $F_{i j}=0$ when $i \neq j$. The integration of trigonometric integrals can be obtained from a mathematics manual.

$$
\begin{aligned}
& F_{11}=\frac{N}{V} 4 R^{2} \int_{0}^{2 \pi} \int_{0}^{\pi} n_{1}^{2} E(n) \sin \gamma d \gamma d \beta=\frac{R^{2} N}{V \pi} \int_{0}^{2 \pi} \int_{0}^{\pi} \cos ^{2}(\gamma)\left(1+\frac{a}{4}(3 \cos (2 \gamma)+1)\right) \sin \gamma d \gamma d \beta \\
& =\frac{R^{2} N}{V \pi} 2 \pi \int_{0}^{\pi} \cos ^{2}(\gamma)\left(1+\frac{a}{4}(3 \cos (2 \gamma)+1)\right) \sin \gamma d \gamma \\
& =\frac{R^{2} N}{V} \int_{0}^{\pi} \cos ^{2}(\gamma)\left(1+\frac{a}{4}(3 \cos (2 \gamma)+1)\right) \sin \gamma d \gamma \\
& =-\frac{R^{2} N}{V \pi} 2 \pi \int_{0}^{\pi} \cos ^{2}(\gamma)\left(1+\frac{3 a}{2} \cos ^{2}(2 \gamma)-\frac{a}{2}\right) d(\cos \gamma) \\
& =\frac{4}{15}(5+2 a) \frac{R^{2} N}{V} \\
& F_{22}=F_{33} \\
& =\frac{N}{V} 4 R^{2} \int_{0}^{2 \pi \pi} \int_{0}^{2} n_{2}^{2} E(n) \sin \gamma d \gamma d \beta=\frac{R^{2} N}{V \pi} \int_{0}^{2 \pi \pi} \int_{0}^{2} \sin ^{2}(\gamma) \cos ^{2}(\beta)\left(1+\frac{a}{4}(3 \cos (2 \gamma)+1)\right) \sin \gamma d \gamma d \beta \\
& =\frac{R^{2} N}{V \pi} \pi \int_{0}^{\pi} \sin ^{3}(\gamma)\left(1+\frac{a}{4}(3 \cos (2 \gamma)+1)\right) d \gamma \\
& =\frac{4}{15}(5-a) \frac{R^{2} N}{V}
\end{aligned}
$$




$$
C_{i j k l}=\frac{N}{V} \int_{0}^{2 \pi} \int_{0}^{\pi} M_{i k} l_{n} l_{q} A_{j n} A_{l q} E(\gamma, \beta) \sin (\gamma) d \gamma d \beta
$$

where $M_{i k}=\frac{1}{k_{n}} n_{i} n_{k}+\frac{1}{k_{s}} s_{i} s_{k}+\frac{1}{k_{s}} t_{i} t_{k}, A_{i j}=F_{i j}^{-1}$, for cross-anisotropic fabric, $A_{i j}=0$, when $i \neq j$.

$$
\begin{aligned}
& A_{11}=\left(F_{11}\right)^{-1}=\left(\frac{4}{15}(5+2 a) \frac{R^{2} N}{V}\right)^{-1} \\
& A_{22}=A_{33}=\left(F_{22}\right)^{-1}=\left(\frac{4}{15}(5-a) \frac{R^{2} N}{V}\right)^{-1} \\
& C_{i j k l}=\frac{N}{V} \int_{0}^{2 \pi} \int_{0}^{2 \pi} M_{i k} l_{n} l_{q} A_{j n} A_{l q} E(\gamma, \beta) \sin (\gamma) d \gamma d \beta \\
& =\frac{N}{V} \int_{0}^{2 \pi \pi} \int_{0}^{\pi} M_{i k} l_{j} l_{l} A_{i j} A_{l l} E(\gamma, \beta) \sin (\gamma) d \gamma d \beta
\end{aligned}
$$

where $j$ and $l$ are not summed. For example,

$$
C_{1111}=\frac{1}{E_{v}}=\frac{N}{V} \frac{4 R^{2}}{4 \pi} \int_{0}^{2 \pi \pi} \int_{0}^{2} n_{1}{ }^{2}\left(\frac{1}{k_{n}} n_{1}{ }^{2}+\frac{1}{k_{s}} s_{1}{ }^{2}+\frac{1}{k_{s}} t_{1}{ }^{2}\right) A_{11}{ }^{2}\left(1+\frac{a}{4}(3 \cos (2 \gamma+1)) \sin (\gamma) d \gamma d \beta\right.
$$

After lengthy derivation, we have

$$
\begin{aligned}
& C_{1111}=\frac{1}{E_{v}}=\frac{15 V}{28 R^{2} N} \frac{1}{(5+2 a)^{2}}\left(\frac{1}{k_{n}}(21+12 a)+\frac{1}{k_{s}}(14+2 a)\right) \\
& C_{1122}=C_{1133} \\
& =\frac{-v_{v h}}{E_{v}}=\frac{N}{V} \frac{4 R^{2}}{4 \pi} \int_{0}^{2 \pi} \int_{0}^{\pi} n_{1} n_{2}\left(\frac{1}{k_{n}} n_{1} n_{2}+\frac{1}{k_{s}} s_{1} s_{2}+\frac{1}{k_{s}} t_{1} t_{2}\right) A_{11} A_{22}\left(1+\frac{a}{4}(3 \cos (2 \gamma+1)) \sin (\gamma) d \gamma d \beta\right. \\
& =\frac{15 V}{28 R^{2} N} \frac{1}{(5-a)(5+2 a)}\left(\frac{1}{k_{n}}(7+a)-\frac{1}{k_{s}}(7+a)\right)
\end{aligned}
$$

Similarly, all components in the matrix can be obtained. 


\section{REFERENCE}

Adu-Osei A., Lytton R.L. and Little D.N. (2000). System identification method for determining the anisotropic resilient properties of unbound aggregates bases. Unbound Aggregates in Road Construction, (pp 249-256). Balkema, Rotterdam.

Allen J.J. (1973). The effect of non-constant lateral pressure on the resilient response of granular materials. Ph.D dissertation. UIUC.

Allen J.J. and Thompson M.R. (1974). Resilient response of granular materials subjected to time dependent lateral stresses. Transportation Research Record, 510, 1-13.

Barden L. and Khayatt A. J. (1966). Incremental strain rate ratios and strength of sand in the triaxial test. Géotechnique, 16(4), 338-357.

Barksdale R.D. and Alba J.L. (1993). Laboratory determination of resilient modulus for flexible pavement design. Interim Report 2, NCHRP, TRB.

Barksdale R.D. and Itani S.Y. (1989). Influence of aggregate shape on base behaviour. Transportation Research Record, 1227, 173-182.

Barksdale R.D., Alba J., Khosla, N.P., Kim R., Lambe P.C. and Rahman. M.S. (1997). Laboratory determination of resilient modulus for flexible pavement design: Final Report

Barksdale R.D., Brown S.F. and Chan F. (1989). Potential benefits of geosynthetics in flexible pavements. NCHRP Report 315, Transportation Research Council, Washington DC.

Bishop A.W. and Henkel D.J. (1957) The measurement of soil properties in the triaxial test. Edward Arnold Ltd., London.

Boyce H.R. (1980). A nonlinear model for the elastic behaviour of granular Materials under Repeated Loading. Proc. of Int. Symp. on Soils under Cyclic And Transient Loading, 285-294.

Brown S.F. (1975). Improved framework for predicting permanent deformation in asphalt layers. Transportation Research Record, 537, 18-30.

Brown S.F. (1996). Soil mechanics in pavement engineering. Géotechnique, 46(3), 383426. 
Brown S.F. and Hyde A.F.L. (1975). Significance of cycling confining stress in repeatedload triaxial testing of granular materials. Transportation Research Record, 537, 49-58.

Brown S.F. and Pain J.W. (1985). Modelling of granular materials in pavements. Transportation Research Record, 1022, 45-51.

Brown S.F. and Selig E.T. (1991). The design of pavement and rail track foundations. Cyclic Loading of Soils: From Theory to Design, (pp. 249-305).

Brown S.F., Tam W.S. and Brunton J.M. (1987). Structural evaluation and overlay design: Analysis and implementation, Proc. $6^{\text {th }}$ Conf. Structural Design of Asphalt Pavements, Ann Arbor.

Casagrande A. and Carrillo N. (1944). Shear failure of anisotropic materials, Proc. Boston Soc. of Civil Eng, 31, 74-87.

Chan F.W.K (1990). Permanent deformation resistence of granular layers in pavements. $\mathrm{Ph} . \mathrm{D}$ thesis, Univeristy of Nottingham, UK.

Chang C.S. (1989). Micromechanical modelling of constitutive relations for granular materials. Micromechanics of Granular Materials, 271-279.

Chaudhary S.K., Kuwano J. and Hayano Y. (2003). Measurement of quasi-elastic stiffness parameters of dense Toyoura Sand in hollow cylinder apparatus and triaxial apparatus with bender elements. Geotechnical Testing Journal, 27(1), 1-13.

Crockford W.W., Bendana L.J., Yang W.S., Rhee S.K. and Sebadheera S.P. (1990). Modelling stress and strain states in pavement structure incorporating thick granular layers. Final Reports, TTI.

Croney D. and Croney, P. (1991) The design and performance of road pavements, $2^{\text {nd }}$ Edition, Mc-Graw Hill Book Company, London,

Dawson A.R. (1995). The unbound granular base. Keynote Paper, Center For Aggregates Research, $3^{\text {rd }}$ Annual Symposium.

Desai C. (2001). Mechanics of Materials and Interfaces: The Disturbed State Concept. CRC Press.

Dunlap W.A. (1963). A report on a mathematical model describing the deformation characteristics of granular materials. Tech. Report No. 1, Project 2-8-62-27, Texas Trans. Inst., Texas AandM University.

Elhannani M. (1991). Modelisation et simulation numerique des chausses souples. $\mathrm{PhD}$ 
Thesis, University of Nantes, France.

Fredlund D.G. and Morgensten N.R. (1977). Stress state variables for unsaturated soils. ASCE Journal of Geotechnical Engineering Division 103, 447-466.

Garg N. and Thompson M.R. (1997). Triaxial characterization of Minnesoda road research project granular materials. Transportation Research Record, 1577, 27-36.

Graham J. and Houlsby G.T. (1983). Anisotropic elasticity of a natural clay. Géotechnique, 33(2), 165-180.

Guo P., Stolle D.F.E. and Liu Y. (2006) Unbound Resilient Modulus Testing of Typical Base, Subbase Materials in Ontario. Final report submitted to the Ministry of Transportation, Ontario.

Hardin B.O. and Blandford G.E. (1989). Elasticity of particulate materials. Journal of Geotechnical. Engineering, ASCE, 115(6), 788-805.

Hardin B.O. and Richart F.E. (1963). Elasticity wave velocities in granular material. Journal of ASCE, ASCE, 89(SM-1), 33-65.

Heydinger A.G., Xie X.L., Randolph B.W. and Guapa J.D. (1996). Analysis of dense and open-graded aggregates. Transportation Research Record, 1547, 1-6.

Hicks, R.G. (1970), Factors Influencing the Resilient Properties of Granular Materials. Ph.D thesis, University of California, Berkeley.

Hicks, R.G. and Monismith, C.L. (1971). Factors influencing the resilient properties of granular materials. Transportation Research Record 45, 15-31.

Hoque E. and Tatsuoka F. (1998). Anisotropy in the elastic deformation of materials. Soils and Foundations, 38(1), 163-179.

Hoque E. and Tatsuoka F. Sato T. (1996). Measurement of anisotropic elastic properties of sand using a large triaxial specimen. Geotechnical Testing Journal, 19(4), 411-420.

Jiang G.L., Tatsuoka F., Flora A. and Koseki, J. (199). Inherent and stress-state iInduced anisotropy in very small strain stiffness of a sandy gravel. Géotechnique, 47(3), 509-521.

Johnson, T. C., Berg, R. L. and Dimillio, A. (1986). Frost action prediction techniques: an overview of research results. Transportation Research Record, 1089, 147-161.

Jouve P. and Elhannani M. (1993). Application des modelles non-liearires au calcul des chausses souples. Bulletin de Liaison des Laboratories des Ponts et Chaussees, 190, 30- 
35.

Karasehin M. and Dawson A.R. (2000). Anisotropic characteristics of granular material. Unbound Aggregates in Road Construction, Balkema, Rotterdam.

Kim S., Little D., Masad E., Lytton R. (2005). Prediction of anisotropic resilient responses for unbound granular layer considering aggregate physical properties and moving wheel load. The $13^{\text {th }}$ ICAR Annual Symposium, Aggregates: Asphalt Concrete, Portland Cement Concrete, Bases and Fines, Austin.

Kohata,Y., Tatsuoka F., Wang L., Jiang G.L., Hoque E. and Kodaka T. (1997). Modelling the nonlinear deformation properties of stiff geomaterials. Géotechnique, 47(3), 563-580.

Kolisoja P. (1997). Resilient Deformation Characteristics of Granular Materials. Ph.D Thesis. Tampere University of Technology.

Koseki J., Tatsuoka F., Munaf Y., Tateyama M. and Kojima K. (1997). A modified procedure to evaluate active earth pressure at high seismic loads. Soils and Foundations, Special Issue, 209-216.

Kuwano R. Connolly T.M. and Jardine R.J. (2000). Anisotropic stiffness measurement in a stress path triaxial cell. Geotechnical Testing Journal, 23(2), 141-157.

Lade P.V., and Nelson R.B. (1987). Modelling the elastic behaviour of granular materials. Int. J. for Numerical and Analytical Methods in Geomechanics, 11, 521-542.

Lam A. (1982). Resilient moduli of flexible pavement materials. MEng thesis, McMaster University.

Lekarp F., Isacsson U., and Dawson A. (2000). State of the art. I: Resilient response of unbound aggregates, Journal of Transportation Engineering, 66-75.

Lings M.L., Penninggon D.S. and Nash D.F.T. (2000). Anisotropic stiffness parameters and their measurement in a stiff natural clay. Géotechnique, 50( 2), 109-125.

Lofti H.A., Schwartz C.W., and Witczak M.W. (1988). Compaction specification for the control of subgrade rutting. Transportation Research Record 1196, TRB.

Love A.E. (1927). A Treatise on the mathematical Theory of Elasticity. Cambridge University Press.

Masad E., Little D. and Lytton R. (2004). Modelling nonlinear anisotropic elastic properties of unbound granular bases using microstructure distribution tensors. International Journal of Geomechanics, 4(4), 254-263. 
NCHRP. (2004). Guide for mechanistic-empirical design of new and rehabilitated pavement structures [online]. Project 1-37A. Transportation Research Board. Available from www.trb.org.mepdg.guide.htm

Nemat-Nasser S. and Hori M (1998). Micromechanics: Overall Properties of Heterogeneous Materials

Oda M., Nemat-Nasser S. and Konish J. (1985). Stress-induced anisotropy in granular masses. Soil Foundation. 25(3), 85-97.

Pappin J.W. and Brown S.F. (1980). Resilient stress strain behaviour of a crushed rock, Proc. Int. Symp. Soils under cyclic and transient loading, Swansea, 1, 169-177.

Park S. and Lytton R.L. (2004). Effect of stress-dependent modulus and Poisson's ratio on structural responses in thin asphalt pavements. Journal of transportation Engineering, 130(3), 387-394.

Pezo R.F. (1993). A general method of reporting resilient modulus tests of soils - A pavement engineer's point of view, $72^{\text {nd }}$ Annual Meeting of TRB.

Pickering D.J. (1970). Anisotropic elastic parameters for soil. Géotechnique, 20(3), 271276.

Plaistow, L.C. (1994). Nonlinear Behaviours of Some Unbound Pavement Aggregates. Ms. Thesis, University of Nottingham, UK.

Rada G. And Witczack M.W. (1981). Comprehensive evaluation of laboratory determination resilient moduli results for granular material. Transportation Research Record, 810, 23-33.

Raymond G.P. 1970. Discussion: Stress and Displacement in a Cross-anisotropic Soil, By Barden 1. Géotechnique, 20(4), 456-458.

Redén, N, (2004). Surface Wave Testing of Pavements. PhD Thesis, Lund University.

Schwarz C.W. 2002. Effect of stress-dependent base layer on the superposition of flexible pavement solutions, The International Journal of Geomechanics, 2, No. 3, pp331-352.

Seed H.B., Chan C.K. and Lee C.E. (1962). Resilience characteristics of subgrade soils and their relation to fatigue failures. Proc. Int. Conf. Structural Design of Asphalt Pavement, Ann Arbor, 611-636.

Selig E.T. (1987). Extensive zone effects on performance of layered systems. 
Geotecnique, 37(3), 247-254.

Seyhan U. and Tuturmluer E. (2005). Anisotropic aggregate base input for mechanistic pavement analysis considering effects of moving wheel load. Journal of Materials in Civil Engineeering, ASCE, 17(5), 505-512.

Schmidt B. (1966). Earth pressures at rest related to stress history. Canadian Geotechnical Journal , 3 (4), 239-242.

Stewart H.E., Selig E.T. and Norman-Gregory, G.M. (1985). Failaure criteria and lateral stresses in track foundations. Transportation Research Record, 1022, 59-64.

Sweere, G.T.H. (1990), Unbound Granular Basis for Roads. Ph.D thesis, University of Delft, The Netherlands.

Stolle D.F.E., Guo P. and Liu Y.(2009) Resilient Modulus Properties of Granular Highway Materials. Canadian Journal of Civil Engineering, 36(4).

Stolle D.F.E., Guo P. and Liu Y. (2005) Unbound Resilient Modulus Testing of Typical Base, Subbase Materials in Ontario: Final report (Phase I) submitted to the Ministry of Transportation, Ontario.

Tam W.A., and Brown, S.F. (1988). Use of the falling weight deflectometer for in situ evaluation of granular materials in pavements. Proc. of $14^{\text {th }}$ ARRB Conf., 14(5), 155163.

Thom, N.H. and Brown, S.F. (1987) Effect of moisture on the structural performance of a crushed-limestone road base. Transportation Research Record, 1121, 50-56.

Thom, N.H. and Brown, S.F. (1989) The Mechanical Properties of Unbound Aggregates from Various Sources, Unbound Aggregates in Roads (pp. 130-142).

Tuturmluer E. and Barkdale R.D. (1995). Behaviour of pavement with granular base prediction and performance. Proc. of Unbound Aggregates in Roads, UNBAR 4.

Tuturmluer E. and Seyhan U. (1997). Anisotropic modelling of granular bases in flexible pavement. Transportation Research Record, 1577, 18-26.

Tuturmluer E. and Seyhan U. (1999). Laboratory determination of anisotropic aggregate resilient moduli using a new innovative test device, determination of resilient modulus for pavement design, TRB Paper.

Tuturmluer E. and Seyhan U. (2000). Effects of fines content on the anisotropic response and characterization of unbound aggregates bases. Unbound Aggregates in Road 
Construction, Balkema, Rotterdam, (pp. 153-160).

Tuturmluer E. and Seyhan U. (2003). Characterization of cross-anisotropic aggregates base behaviour from stress path tests. Recent Advances in Material Characterization and Modelling of Pavement Syestems (pp. 18-34). ASCE.

Tuturmluer E., Little D.N., and Kim S. (2003). A validated model for predicting field performance of aggregates base courses. Transportation Research Record 1837, 41-49.

Uzan J. (1985). Characterization of granular material. Transportation Research Record, $1022,52-59$.

Uzan J. (1992). Resilient characterization of pavement material. Int. Journal for Numerical and Analytical Methods in Geomechanics, 16, 453-459.

Van Niekerk A.A. (2002). Mechanical Behaviour and Performance of Granular Bases and Sub-Bases in Pavements. Ph.D. Thesis. Delft University of Technology.

Van Niekerk, A.A., Houben, L.J.M., and Molenaar, A.A.A. (1998). Estimation of mechanical behaviour of unbound road building materials from physical material properties. Proc. $5^{\text {th }}$ Int. Conf. on the Bearing Capacity of Roads and Airfields, 3, 12211233.

Witczak M.W., Qi X., and Mirza M.W. (1995). Use of nonlinear subgrade modulus in AASHTO design procedure. Journal of Transportation, 121 (3), ASCE.

Yau A. and Von Qu, H.L. (2002). Study of LTPP Laboratory Resilient Modulus Test Data and Response Characteristics: Final report. Federal Highway Administration, Publication No. FHWA-RD-02-051.

Zdravkovic L. and Jardine R.J. (1997). Some anisotropic stiffness characteristics of silt under general stress conditions. Géotechnique, 47(3), 407-437. 